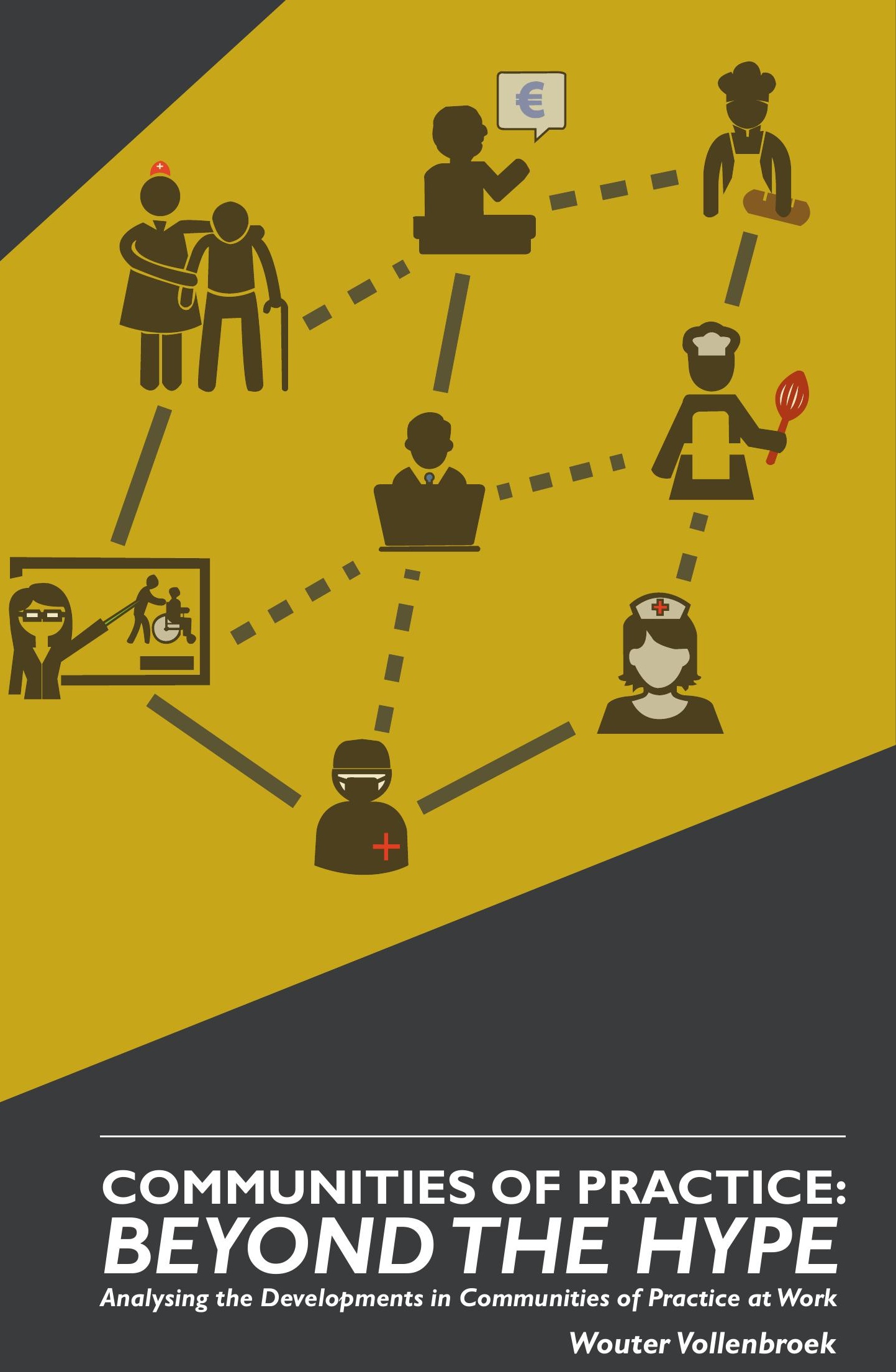




\section{COMMUNITIES OF PRACTICE: BEYOND THE HYPE}

Analysing the Developments in Communities of Practice at Work

WOUTER VOLLENBROEK 


\title{
COMMUNITIES OF PRACTICE: BEYOND THE HYPE \\ Analysing the Developments in Communities of Practice at Work
}

\section{PROEFSCHRIFT}

\author{
ter verkrijging van \\ de graad van doctor aan de Universiteit Twente, \\ op gezag van de rector magnificus, \\ prof.dr.T.T.M. Palstra, \\ volgens besluit van het College voor Promoties \\ in het openbaar te verdedigen \\ op donderdag 17 oktober 2019 om l 6:45 uur
}

door

\section{Wouter Bernardus Vollenbroek}

geboren op II december 1988

te Oldenzaal (Nederland) 
Dit proefschrift is goedgekeurd door:

de promotoren:

prof.dr.J.A.G.M. van Dijk \& prof.dr.W.E. Ebbers

de co-promotor:

dr. S.A. de Vries

Cover design:Wouter B.Vollenbroek

Some icons on the cover page are based on the work of freepik from www.flaticon.com Printed by: Ipskamp Printing - The Netherlands

Lay-out: Wouter B.Vollenbroek

ISBN: 978-90-365-4820-5

DOI: $10.3990 / 1.9789036548205$

(C) 2019 The Netherlands. All rights reserved. No parts of this thesis may be reproduced, stored in a retrieval system or transmitted in any form or by any means without permission of the author.Alle rechten voorbehouden. Niets uit deze uitgave mag worden vermenigvuldigd, in enige vorm of op enige wijze, zonder voorafgaande schriftelijke toestemming van de auteur 


\section{PROMOTIE COMMISSIE:}

Voorzitter/secretaris

Promotoren

Co-promotor

Leden
prof.dr.T.A.J. Toonen

prof.dr.J.A.G.M. van Dijk \&

prof.dr.W.E. Ebbers

dr. S.A. de Vries

prof.dr. L.A.L. van de Wijngaert

prof.dr. B.J. van Hooff

prof.dr. ir. B.P.Veldkamp

prof.dr. M.D.T. de Jong 




\section{Table of Contents}

9 Chapter I - General introduction

PART I: MODELLING DEVELOPMENT IN COMMUNITIES OF PRACTICE

$21 \quad$ Chapter 2 - Key Concepts in Community Development

39 Chapter 3 - Community of Practice Development Model

PART II: RESEARCH METHODOLOGY

55 Chapter 4 - Selection of Cases

65 Chapter 5 - Community Monitoring and Evaluation Methodology

PART III: INDIVIDUAL SOCIAL BEHAVIOUR IN COMMUNITIES OF PRACTICE

77 Chapter 6 - Individual Factors Driving Social Behaviour in Communities of Practice

99 Chapter 7 - Organisational Factors Driving Social Behaviour in Communities of Practice

II 3 Chapter 8 - Community Factors Driving Social Behaviour in Communities of Practice

PART IV: GROUP BEHAVIOUR IN COMMUNITIES OF PRACTICE

I27 Chapter 9 - Development Phases of Communities of Practice

I5I Chapter 10 - Individual Social Network Positions in Communities of Practice

169 Chapter H - Social Interaction Patterns in Communities of Practice

PARTV: CONCLUSIONS \& DISCUSSION

189 Chapter 12 - Conclusions and Discussion

203 References

235 Appendices

27I Samenvatting (Dutch Summary)

278 Dankwoord (Dutch Acknowledgements)

280 About the author 



\section{1 \\ General Introduction}

\section{I.I Introduction}

Rapid technological advances, ever-changing markets in which increasingly high-quality standards are set, globalization and a growing need for individual personal development (Bender \& Fish, 2009; Sessa \& London, 20I5;Timans, Ahaus, van Solingen, Kumar, \& Antony, 20I6) are only four developments which have a major impact on the way in which organisations shape themselves. The result is an organisation that continuously adapts to changing circumstances at the individual, organisational and group levels, with an impact on the strategic and social movements in the organisation (Sessa \& London, 2015). This tendency has been visible for a couple of years now and has resulted in different organisational responses.

A common answer from a growing group of organisations is a transition towards a knowledgedriven business strategy (Nielsen, 20I8), with more attention given to individuals' continuing professional development (Andersson, Formica, \& Curley, 2009) and social learning (Wenger, 2008). One method for combining the development of a knowledge-driven business strategy with individual continuous professionalization and social learning is a community of practice. Initially, a community of practice (CoP) was defined as a group of people who share a craft or profession (Lave \& Wenger, I99I). In this dissertation, a definition for a CoP, mainly focused on organisational practices, has been formulated that better fits the current spirit of the times and the state of digital developments. The CoP is defined as "A community of practice is a social network where people in an organisational context come together around a common topic, passion or interest and regularly interact on- and offline with a focus on knowledge management, innovation, learning and social networking". Using the concept of 'communities of practice' (CoPs), Lave \& Wenger (199I) changed the way in which we look at personal and organisational learning from personal to situated and social learning. In fact, it is working with others that forms a fundamental process by which individuals learn (Wenger, 1999).

We especially focus on the communities of practice within the context of an organisation. The main reason for this is that these types of social systems are receiving increasing attention within organisational management. As they can fulfil the need to optimize knowledge management (KM) and cooperation between employees within the organisation. Obviously, CoPs are not only focused on the boundaries of their own organisation, they also go-purposefully or organically-beyond their own borders. However, as mentioned above, in this research, we focus mainly on those CoPs that are initially internally oriented. The underlying procedures that resulted in the definition used in this dissertation are further explained in Chapter 2, which aims to define the key concepts in this dissertation. This predominantly exploratory dissertation asks the questions of how and why knowledge management and strategies for the continuing professional development of individuals and groups in CoPs develop in the face of technological markets and societal challenges. Answering these questions is expected to improve our understanding of the developments in CoPs and to improve our understanding of a methodology for monitoring and evaluating these developments. The following three 
paragraphs outline the underlying motivations for using CoPs and conducting research into their development based on three levels: organisation level, individual level, and collective level.

\section{I.2. Knowledge management in organisations}

Understanding that knowledge is an organisation's most valuable resource - as it represents assets, operational routines, and creative processes that are hard to imitate (Grant, 1996; Liebeskind, 1996) - makes optimal knowledge management one of the most important activities within an organisation. To compete effectively, knowledge-driven organisations must utilize their existing knowledge and generate new knowledge that positions themselves in a favourite position in their chosen markets (Cohen \& Levinthal, 1990). A commonly used definition of knowledge management is "improv[ing] organisational performance by enabling individuals to capture, share, and apply their collective knowledge to make open decisions...in real time" (Smith \& Farquhar, 2000, p. I7.). For an organisation, the goal of knowledge management is "to become aware of its knowledge, individually and collectively, and to shape itself so that it makes the most effective and efficient use of the knowledge it has or can obtain" (Bennet \& Bennet, 2003, p.440). Many knowledge-driven organisations have started initiatives such as intranets, regular meetings or CoPs to optimize the knowledge exchange within their organisation (Bender \& Fish, 2009).

Knowledge can be effectively used in multiple ways, for instance: for discussions, for problem solving and for teaching and mentoring. As discussed by previous researchers, new knowledge always begins with the individual but ends within an organisation (Nonaka, 1994). An individual's personal knowledge is transformed into organisational knowledge that is valuable to the company. According to Senge (1990, p.12), people truly change when they learn new skills and learn to apply these skills in their work. The personal development that arises not only has a positive effect on the development of the individual but also on the performance of the organisation. Slater \& Narver (2006) describe effective knowledge-driven organisations as organisations that are configurations of management practices that facilitate the development of the knowledge that becomes the basis for competitive advantage. Such an organisation is not merely an information-processing machine, but an entity that creates knowledge through action and interaction (Nonaka \& Toyama, 2003).

An effective organisation continuously tries to optimally utilize the knowledge and skills of its employees to realize competitive advantage. Guptill (2005) highlighted the optimal use of employees as one of the most effective methods to build knowledge management. The method introduced by Wenger (2004) listens to the term community of practice which is a social factory of knowledge acquisition and transfer. This knowledge management approach based on CoPs must lead to a fundamental transformation of knowledge-driven organisations. Such an approach assumes that knowledge is the property of the employees and that the role of management is to make it possible for the employees to act as the managers of their own knowledge. Brown \& Duguid (1991) argue that, through their constant adaptation to changing membership and changing circumstances, evolving CoPs are significant sites for all forms of innovation. However, in depth insight in the role of CoPs in this transformation is needed (Christson \& Adedoyin, 2016;Wenger \& Snyder, 2000).

\section{I.3. Individuals' Continuing Professional Development}

Knowledge management is of major importance for organisations, and encouraging employees 
to exchange knowledge and optimize their cooperation with peers is of crucial importance in this respect (Chiu, Hsu, \& Wang, 2006). Optimal knowledge management is about getting individuals together to create success in, for example, CoPs. However, the realization of the optimal exchange of knowledge and cooperation is not only in the interest of the organisation. Contemporary professionals also want to develop themselves continuously through broadening or deepening their knowledge and skills. Competencies that are inter-organisationally applicable make professionals more adaptive to the changing situations of today, attractive to other organisations and able to improve their own work practices. The new way of working in society puts stress on the need for lifelong learning and the need to continuously adapt, change, and learn new skills, very often on site while carrying out the job (Gee, Hull \& Lankshear, 20I8): "The ability to access relevant information and harness the resources offered by the views and opinions of others have become important skills, particularly as the need for lifelong learning, both formal and informal, is increasingly recognized by individuals, organisations and institutions" (Goldie, 2016, p. 1064).

To make a sustainable contribution to society and their (future) work field, it is crucial for professionals to continue developing and adapting to changes in society, their field of work and the use of (new) technologies. Continuous professional development plays an increasingly fundamental role in this process. It is part of the professional development of the professionals in an organisation. Thus, this is a more professional-centred perspective focused on an individual's learning. Continuous professional development encompasses both formal and informal knowledge sharing approaches that can result in positive changes in the knowledge, skills, attitudes, and behaviour of individuals (Hew \& Hara, 2007). The existing initiatives in organisations are often organized in professional development programmes formalized in, for example, formal training activities at unfavourable moments (Duncan-Howell, 2007). The connectivism approach (Siemens, 2005) is more flexible in that matter since it views learning as a network phenomenon influenced by technology and socialization. Professionals are increasingly being able to learn in their own time and at their own place independently from each other, but nevertheless with others (Jin, 2002). Despite the extensive research, adequate insight into the background of individual behaviour within a CoP is lacking.

\section{I.4. Collective knowledge mechanisms in organisations}

Building on the idea behind the connectivism, organisations are increasingly trying to create an atmosphere where individuals, departments and groups can work together. Organisations are increasingly trying to support these employees to improve cooperation, to increase their competitive position, to improve their innovative capacity and to be able to operate more efficiently and effectively in a continuously changing market (Tsvyk \& Tsvyk, 20I8). To create such an atmosphere, organisations often initiate, for example, self-organizing teams, SCRUM teams and CoPs. A common denominator in these three examples seems to be the term 'shared'. In each of these examples, the focus is on creating shared goals and purposes, a shared identity that stimulates commitment, attachment and involvement and a shared language that improves mutual understanding.

Especially for the sake of knowledge management; collaboration and social bonding in CoPs are important concepts. By initiating a CoP, organisations are increasingly opting for online community software to take full advantage of the possibilities to reduce social distance between employees and thereby optimize knowledge exchange (Castillo De Mesa, Gómez Jacinto, López Peláez, \& Palma García, 2019). A fundamental precondition for calling a CoP a CoP, is 
the perception of social bonding in the workplace (Ifinedo, 20I4) in the form of attachment, commitment and involvement with each other and the organisation, and mutual personal norms (Hirschi, 2002). Hooff \& Ridder (2004) and Kraut \& Resnick (20I I) emphasize the relevance of having employees who are committed to the organisation as an important indicator for knowledge-donating activities, a situation that will most likely also apply to CoPs. In particular, the role of computer-mediated communication seems to have a positive influence on this.

Current computer-mediated communication developments not only contribute to optimizing knowledge development within organisations but also provide fertile ground for social interactions between individuals. Media increasingly serve as the means that allow us to come together in some form of community and offer us a sense of belonging (Littlejohn, Foss \& Oetzel, 2017). Organisations try to take full advantage of this by purchasing a licence for the use of online community software that enables its employees to collaborate, exchange knowledge and engage in other types of social interaction. In the implementation of these platforms, various interventions are carried out to realize the sense of community among the users, with varying success. In some organisations, the sense of community increases, since barriers between departments can be dismantled, but in other organisations, new barriers are raised (Ardichvili, Page, \&Wentling, 2003). In this research, it is the ambition to contribute to filling the lack of expertise for building and maintaining CoPs (Wenger \& Snyder, 2000).

Following the motivations, further intensifying cooperation, knowledge exchange and other mutual contact forms receive increasing attention from organisations, individuals and the collective. Supporting the development of CoPs from these three perspectives is therefore a logical step in this dissertation. These three perspectives come together to a greater or lesser degree on the two levels: individual social interaction and group interaction (Kraut \& Resnick, 20II)

The - predominantly - exploratory research described in this dissertation focuses on what individual, organisational and collective factors affect individual social behaviour in CoPs (Part III), how group behaviour develops (Part IV); and what a useful community monitoring and evaluation methodology is for following the developments of CoPs (Part II up to including Part IV).

\section{I.5. Scope, research goals and questions and outline of the dissertation}

To address the gaps and ambitions outlined above, this dissertation aims to explore the development in and of CoPs from various perspectives. It aims to develop a theoretical model to understand the social behaviour of individuals and groups and translate these findings into implications for the development, implementation and policy-making processes of CoPs. As known, a growing tendency of knowledge-driven organisations focus specifically on spreading and increasing knowledge within their organisations and on the acquisition of knowledge from outside the organisation can be identified in today's knowledge society. Due to the continuous urge for efficient, effective and innovative organizing and development, it is crucial to be open to collaboration and knowledge exchange as an individual, an organisation and a discipline in general.The transition a few decades ago to a knowledge society has ensured that knowledgedriven organisations increasingly focus their pillars on creating, using, and expanding the intellectual capital of individuals more explicitly. In many knowledge-driven organisations, it is therefore encouraged to share knowledge to indirectly strengthen the knowledge society. In this paragraph, the main goals, the research outline and research questions are introduced. 
The main objective of this predominantly exploratory dissertation is to promote the development of knowledge and understanding regarding the development of CoPs, which will be captured in the general research question:

How and why do communities of practice in organisations develop?

To get an answer to this general research question, it has been subdivided into three research goals. First, identify factors that affect the development of CoPs, second, provide insight into group behaviour in CoPs, and finally, design a community monitoring and evaluation methodology. By reaching these goals, this research is set to provide community-managers and organisational managers with design knowledge and knowledge about the behaviour of members in CoPs that may help them in directing their CoPs in a preferred direction.

Based on these three goals, the entire dissertation revolves around the following three research questions:

- What individual, organisational and community factors affect the social behaviour of community members in a community of practice?

- How does group behaviour develop in a community of practice?

- What is a relevant methodology for the monitoring and evaluation of individual and group behaviour in communities of practice?

The thesis has been divided into five parts to answering the three research questions and achieve the goals of this dissertation. These five parts are: (I) modelling CoP development, (II) research methodology, (III) individual social behaviour in CoPs, (IV) group behaviour in CoPs, and $(\mathrm{V})$ conclusions and discussion. The first part forms the theoretical foundation for the first two research questions, and the second part forms the introduction for the last research question, which is subsequently operationalized in the methodological parts of each chapter; this part also forms the basis for constructing the community monitoring and evaluation methodology (CMEM). Part III will revolve around the first research question, and the second research question will be the basis for the fourth part. The final part includes the conclusions and discussion and will provide an answer to all of the research questions. Generally speaking, it is the ambition to model behaviour in CoPs and develop a methodology that is expected to contribute to the expertise in building and maintaining a CoP.

\section{I.5.I. Part I: Modelling Development in Communities of Practice}

Research into the concept of CoPs as a means of fostering cooperation, sharing knowledge, and increasing social cohesion has been around for a while. For decades, many academics have conducted research on all kinds of perspectives for analysing CoPs. Developing CoPs in organisations requires a clear understanding of the processes (Tremblay \& Psyché, 20I2), structures (Hildreth \& Kimble, 2004) and supporting variables that affect this development (Ardichvili, 2008). In this part of the dissertation, the focus is on sketching a framework for research into the development of CoPs.

The general aim in Chapter $\mathbf{2}$ is to define the central concepts relevant to understanding the developments in CoPs. Academic attention for developments of CoPs provides a variety 
of concepts as well as interpretations of these concepts. In this chapter, we define the most important concepts relevant to this research project.

The theoretical foundation for this dissertation will be built in Chapter 3. The conceptual model designed to evaluate individual social behaviour in a CoP is based on the existing motivation, opportunity and ability model (Maclnnis \& Jaworski, 1989). The original MOA model was intended to explain consumers' responses to advertisements. However, in the past decades, it has evolved into a broadly applicable model in, for example, knowledge management , and for other types of social behaviour in online communities (Gruen, Osmonbekov, \& Czaplewski, 2007; Hughes, 2007). Even though the MOA model is an accurate model (Weick, 1979), we have adapted the model at various levels to ensure that it is more applicable for the evaluation of individual and group behaviour in CoPs. The most important addition is the introduction of a fourth concept - communality - which provides insight into the vital preconditions for the development of a sense of community. These four variables together form the MOAC model, the model that will form an important part of the evaluation of individual and group behaviour in the CoPs central in this dissertation. The first sub-question in this dissertation has been formulated for Chapter 3: Which factors potentially affect the social behaviour of community members in communities of practice?

\section{I.5.2. Part II: Research Methodology}

In this dissertation, a comprehensive set of research methods are used to improve our insight into the developments in CoPs. Both the perceptions of community members and the actual behaviour play an important role in this process.

Chapter 4 presents the overarching cases in this dissertation. In this chapter, the cases will be described and evaluated based on predefined criteria evaluated in Chapters 2 and 3. The bases of the three criteria have their roots in organisational culture, the goals and objectives organisations have with CoPs, and the implementation strategies that exists in CoPs. The cases defined in this chapter form the context of the research in almost every chapter (except for chapter 9 in which three extra cases have been added).

In this research, it is possible to not only treat behaviour from the perception of the individual but also to connect it with actual behaviour. This makes the methodology of the research used in this dissertation unique in its nature, especially when focusing on the analysis of CoP developments. In Chapter 5, the different methodologies in this dissertation are introduced and substantiated. This chapter describes the first steps towards the design of the community monitoring and evaluation methodology (CMEM). In Parts III and IV, parts of the methodology will be applied to provide insight into the CoP developments and factors which affect these developments.

\section{I.5.3. Part III: Individual Social Behaviour in Communities of Practice}

People's commitment to CoPs initiated in work contexts depends on several factors that have been intensively studied in the academic world (e.g., Ardichvili, 2008; Siemsen, Roth, \& Balasubramanian, 2008). In general, these factors can be categorized into three groups: individual factors, organisational factors and community factors. In this part of the dissertation, the focus is on individual testing the relationship between individual factors, organisational factors and community factors with individual social behaviour of community members in CoPs. 
Chapter 6 presents survey research with a sample of professionals from four organisations who are already a member of a CoP.The four CoPs of these four organisations are comparable in nature due to their use of the same online community software. A selection interview was conducted with the four organisations to determine whether their initiatives comply with our definition of a CoP.The focus in this chapter is on the individual factors (motivation and ability) from the MOAC model that affect an individual's social behaviour in a CoP.The following subquestion has been formulated for this chapter:What are the reasons, reports and perceptions of professionals about their knowledge acquisition and social-interaction behaviour that affect participating in Communities of Practice?

In Chapter 7, it is presumed that an individual's perception of organisation-supported initiatives contributes to his or her actual social behaviour in CoPs. The attention that organisational management devotes to the success of a CoP is often seen as an important indicator for the level of individual social behaviour. In Chapter 6, the focus is on examining the impact of individual factors on social behaviour in CoPs. In Chapter 7, the focus is on testing the impact of the organisational factors (opportunities) on an individual's social behaviour in CoPs. This survey-based study forms part of a larger study of factors that influence individual social behaviour in CoPs. The related sub-question to be answered in this chapter is as follows:Which organisational factors do professionals report as potential drivers and barriers towards their social behaviour in CoPs?

In Chapter 8, the last fundamental component of the MOAC framework has been studied: the role of communality and its latent variables on the social behaviour of professionals in CoPs. The central sub-question in this chapter is as follows: Which community factors affect the social behaviour of professionals in a community of practice?

\section{I.5.4. Part IV: Group Behaviour in Communities of Practice}

CoPs-initiated by organisations or individuals-ultimately revolve around collective development based on group behaviour. The community ultimately determines whether there is enough support for this method of improving cooperation, knowledge management or other types of social interaction. An inactive community soon resembles a "cyber ghost town" (Phang, Kankanhalli, \& Sabherwal, 2009) in which only sporadic activity is visible. It is therefore vital that community managers and other stakeholders contribute in a meaningful way to the development of the CoPs. To realize this, it is fundamental to be well informed and to keep abreast of the developments within a CoP. In this part of the research project, community development is therefore considered from the viewpoint of group behaviour. In the end, individuals in an organisation are whole systems themselves that are part of other systems such as teams, groups, and communities (Sessa \& London, 2015). This part is subdivided into three distinctive studies, all of which make a unique contribution to our understanding of group behaviour in CoPs.

In Chapter 9, the general aim is to analyse the development of CoPs from a data-driven perspective. Web-based social interaction data collected from a total of seven CoPs using the same online community software provider will be used as the basis for visualizing and analysing community development patterns. Web-based social interaction data are those data that represent an individual's behaviour in an online environment, such as creating a post, commenting on a post, or appreciating a post. The community development patterns will be normalized to reduce the mutual differences in frequency and maturity and to create comparable cases. These 
seven cases are formed by the four cases described in Chapter 4 and three other cases that use the same online community software. The sub-question formulated for this chapter is as follows: Which aspects of community development patterns characterize the development of a community of practice?

Individual CoP network positions are affected by an individual's conscious or unconscious social behaviour in a social network. In Chapter 10, the primary focus is on the MOAC factors introduced in Chapter 3 that affect these positions. In Chapters 6, 7 and 8, the MOAC framework will separated into factors from the individual, organisational and collective levels. In this chapter and in Chapter II, the MOAC framework is used as an overarching model to explain individual behaviour in the social networks that form the foundations of CoPs, so the relationship between the motivation, opportunities, ability and communality with the network positions. The main sub-question that is connected to this chapter is as follows: Which factors affect the formation of social network positions in CoPs?

The final chapter in this part-Chapter I I-aims to evaluate social interaction patterns in CoPs by combining subjectivity with objectivity. This approach corresponds to a matching subquestion: What structural processes and individual-level factors shape the network structures in communities of practice? Data from the survey will be merged with the web-based social interaction data. This allows us not only to recognize social interaction patterns but also to give explanations for these patterns.

\section{I.5.5. Part V: Conclusions and discussion}

Chapter $\mathbf{I} \mathbf{2}$ is reserved for the general discussion of the dissertation, the overall conclusions and the implications for practice. The chapter concludes on the key findings described in each chapter, discusses the main limitations, and suggests directions for future research, provide both theoretical and practical implications and closes with a discussion.

In Figure I.I on the next page, an overview of the interrelationships between the chapters in this dissertation is given. The studies in this dissertation address the need to create knowledge and understanding in the development of CoPs from various perspectives. 


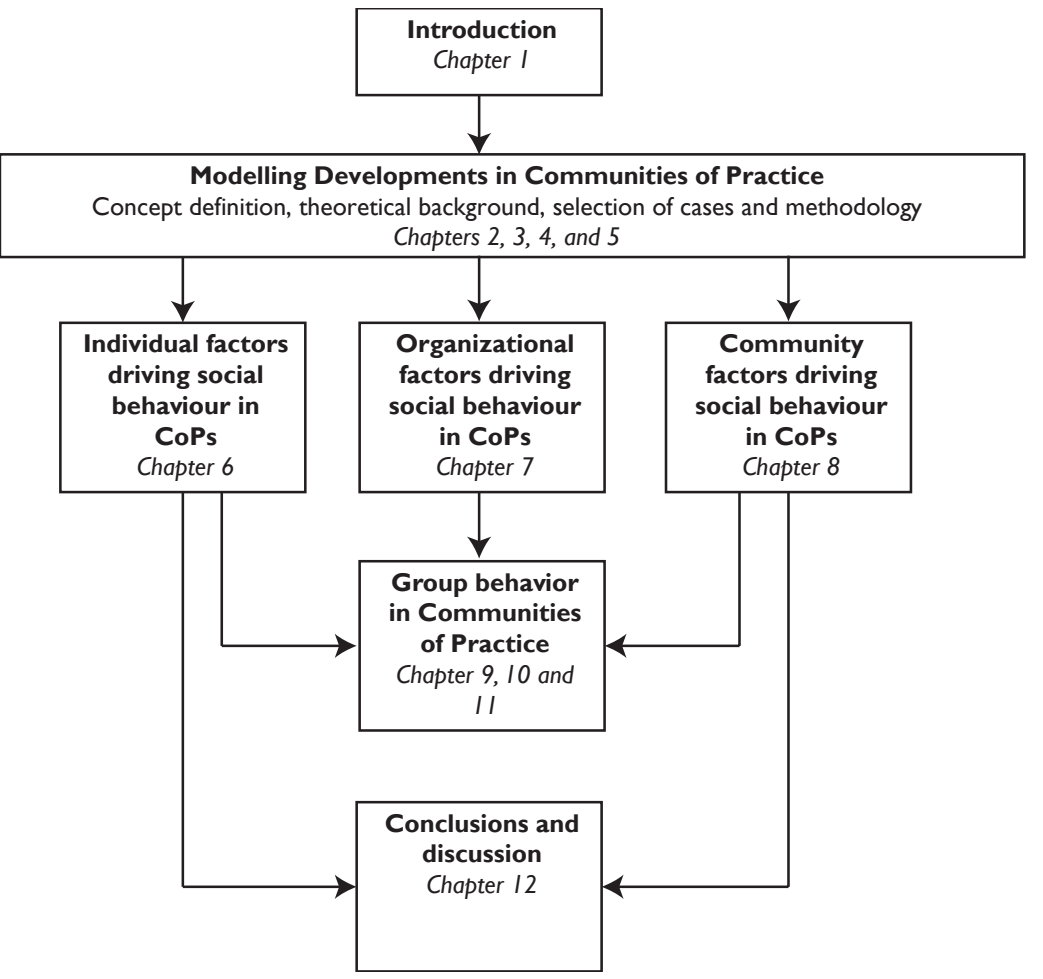

Figure I.I. Coherence between chapters 



\section{PART I:}

\section{MODELLING}

\section{DEVELOPMENT IN \\ COMMUNITIES OF}

PRACTICE
The first chapter of this dissertation is reserved for the introduction of the research project. In the first part of this dissertation, consisting of the Chapters 2 and 3 , special attention is given to modelling the developments in communities of practice.

In Chapter 2, the key concepts relevant in this dissertation are introduced. These concepts constitute the guidelines for reading this dissertation.

In Chapter 3, the theoretical foundations for the framework used in this dissertation is given. The basis for this framework lies in the Motivation, Opportunity, Ability model that primarily focuses on explaining behaviour by means of individual's motivation and contextual factors such as opportunity and ability. In this dissertation, we extend the contextual factors with a fourth variable: communality. 



\section{2 \\ Key Concepts in Community Development}

\section{I. Introduction}

This chapter addresses the central concepts in this dissertation. A common understanding of the terms that are central to this research is crucial for an optimal interpretation of the research directions. CoPs can be viewed from an enormous range of perspectives. For example, by means of development, evolution, intention to act, actual behaviour, impact, value, roles and structures. All of these terms and perspectives are interpreted in different ways. The most important concepts relevant to this dissertation are introduced in this chapter. This chapter therefore outlines the framework for the core components in this research. Since the general objective of this dissertation is to analyse and examine developments in CoPs within work contexts, the following concepts are important to define in advance: continuing professional development, social interaction, CoPs, community infrastructure, community development, community foundation framework (organisational objectives, implementation strategy, organisational culture) and community member roles (community member roles \& community member positions).

The focus in this chapter is therefore primarily on describing the main concepts in this dissertation. The theoretical models in this research will be discussed in chapter 3 .

\subsection{What is Continuing Professional Development?}

The ongoing development and need for development of individual professionals in organisations is increasingly recognized by organisational management (Hoffmann, Desha, \& Verrall, 20II; Jiménez-Zarco, González-González, Saigí-Rubió, \& Torrent-Sellens, 20I4; Rodman, 2018). Sessa \& London (2015) recognize the importance of the individual, the organisation and the group when speaking about continuous learning in organisations. According to the same authors, learning at the individual, group and organisational levels can be adaptive, generative and/or transformative. Adaptive learning occurs when one reacts to changing circumstances in the environment, generative learning aims to create new knowledge and conditions and transformative learning focuses on the creation and application of frame-breaking ideas and radically new conditions. It is up to the organisation whether its employees have the 'freedom' to perform in a manner that contributes to one of these three knowledge creation processes.

Organisations need professionals who can perform in a meaningful manner and who continuously update the knowledge, skills and attitudes that are crucial for performing their current and future jobs (Webster-Wright, 2009). To realize this, organisations have introduced several continuing professional development (CPD) programmes and initiatives. In the past, CPD consisted mainly of formal learning activities such as meetings, lectures and the pursuit of obtaining formal degrees (Barry, Kuijer-Siebelink, Nieuwenhuis, \& Scherpbier-de Haan, 20I7), but with the introduction of digital applications and developments at the level of professional growth, the possibilities for CPD have also increased. Nowadays, continuing professional development is 
often used synonymously with the terms lifelong learning and continuing education. However, Vries (2003) sees CPD as a distinctive concept where professional development occurs due to formal and informal learning in the course of practice. Formal and informal learning occur synergistically and are therefore of vital importance for the development of the professional. Thus, CPD is not only about learning or education but also about the continuing process of individual professional development. Following the line of thought of Webster-Wright (2009) leads us to the idea that individual and group behaviour in professional practice can in general be viewed as "moment-by-moment" continuing learning. The individual is thus in a continuous process of learning and experiencing, which leads to the development of new or improved insights. Developments manifest themselves in different forms, for example, in organisationspecific and subject-specific knowledge but also in professional networks.

However, in literature, the transfer of subject-specific knowledge and organisation-specific knowledge are identified as important focus areas with increasingly specific attention for developments professional networks. Nonaka, Toyama, \& Konno (2000) concretize knowledge by referring to 'stocks of knowledge' and 'specific resources' and stress the significance of the assets for individual and organisational success. These assets can be the professionals themselves, documents, methods or best practices. Effective CPD indicates professional development that secures the optimal development of these knowledge assets, which ultimately has a positive impact on professional products and services (Vries, 2003). The aim is to achieve optimal cooperation and therefore knowledge exchange and social interaction. Lave \& Wenger (I99I) recognize the importance of a network or team in the implementation and adoption of new and/or updated knowledge in practice. These authors propose that learning is not an individual activity with a predefined beginning and end but is pervasive, ongoing, and part of everyday life. The term used to define the learning process that an individual follows is described as 'situated learning'. Situated learning complements formal education and incorporates, for example, adult learning, problem-based learning and the pedagogy of continuous professional development. Professionals increasingly use their peers and acquaintances to increase their own level of knowledge.

In short, the concept of CPD is defined in this research project as a process focusing on the continuing development of individual knowledge, skills and attitudes that are beneficial for both the professional and the organisation and in which formal and informal learning practices are intertwined.

\subsection{What is Social Interaction?}

Continuous professional development is often affected by the knowledge development processes in which synergy between individuals is optimally utilized and enlarged.A crucial concept used to describe this synergism is social interaction. The concept of social interaction is an important one in both the sociological and the communicational sciences. This largely comes forth in a common definition used to characterize the domain of sociology. Sociology is defined as the systematic and scientific study of human behaviour, social groups, and society (Thompson, Hickey \& Thompson, 2016) and is thus interested in individual actions but also in the social behaviour between individuals. Communication scholars Conrad \& Poole (1998) and Dainton \& Zelley (2005) also introduced a widely acknowledged definition to the communication domain in which social interaction is centrally positioned. These authors define communication as "the process by which people interactively create, sustain, and manage meaning". The role of social interaction is crucial in both domains because a corresponding definition is needed. However, 
academics approach the concept of social interaction from different angles.

Numerous theories have been developed to describe social interaction and its role and impact. Most of them study the ways that people engage with one another (a.o. Bagozzi, Dholakia, \& Pearo, 2007; Curran \& Murphy, 2009), for example, by identifying and analysing patterns that contribute to the development of theories aimed at describing and predicting human behaviour (Bode, Sutton, Lacey, Fennell, \& Leonards, 2017). Weber, a prominent sociologist, focused his research on social interaction and identified two components in social behaviour. The first is the action or the behaviour itself, and the second is the meaning that the actor attaches to his or her behaviour. That meaning-also defined as orientation-is "the actual interpersonal practices that actors use to develop orientations towards each other" (Turner, 1988, p.5). It is that knowledge of another person who is affected that makes an action or interaction social. This more individually focused perspective has been increasingly supplemented with additional collective or networked perspectives. Studying whole groups of people rather than just individual interactions offers opportunities to understand the connections between the members of a group. These connections can be conversations, formal or informal social relations, written or online communication or any other type of social exchange between people.

Other attempts to define the concept of social interaction have been made by Nash \& Calonico (1996) and Campo \& Chaudhury (2012). These authors define social interactions as a dynamic interplay between two or more individuals in which a process of communication and mutual influence involve the contact between two or more minds. Turner (1988) defines this as a more mutually influential process in which the behaviours of actors influence and are consciously reorganized by the behaviours of another actor. In this interplay between actors, different types of social interactions occur. For example, Goffman (1959) studied social interactions from a sociological perspective and identified five different types: exchange, competition, cooperation, conflict and coercion. Zboralski, Salomo, \& Gemuenden (2006) use a more rudimentary division of the distinction between two types of social interaction: information exchange and networking.

All in all, each of these types of social interaction aligns with an individual's desire to control the impressions that other people form about that individual (Nistor, Daxecker, Stanciu, \& Diekamp, 2015). Therefore, the main reason to participate in online social platforms is to present oneself in cyberspace. Typically, this is done through self-disclosure, which implies the conscious and unconscious revelation of personal information (e.g., thoughts, knowledge, likes, appreciations) that is consistent with the image one would like to present (Kaplan \& Haenlein, 2010). If one wants to improve the image that others have of oneself, one must act according to the way one wants to be seen. If one wants to be an expert in a specific area, one could decide to, for example, proactively share knowledge or give positive critical comments on the contributions of others. If one wants to be a connector, one could choose to connect with others oneself and connect others with others.

The predominantly data-driven background of this dissertation leads to the decision to follow the definitions by Nash \& Calonico (1996) and Campo \& Chaudhury (20I2). These authors operationalized social interaction by focusing on types of social interactions and the identification of specific patterns. In organisational environments, these - for example community development and social interaction - patterns can be seen as social groupings with relatively stable patterns of interaction over time (Tichy, Tushman, Fombrun, \& Tushman, 1979). The concept of interaction patterns describes the search for underlying patterns in these social interactions. The social network approach, which is often used to identify these social 
interaction patterns, has the basic assumption that "the social structure of any complex system consists of stable patterns of repeated interactions connecting social actors with one another" (Vries, 2003, p.2 18). Panzarasa, Opsahl, \& Carley (2009) point to the fact that many researchers overlook the relevance of interaction patterns. This is due to the earlier imperfections in the data collection of network-related data that are exemplified in, for example, informant inaccuracy and labour intensiveness. Technological advances have resulted in automatically collected and stored web-based social interaction data in digital software. This has paved the way for research into objective, accessible and comprehensive datasets. The social interactions that take place organically or that are orchestrated within the digital environments of an organisation can therefore be made easily understandable. The rise of CoPs-supported by online community software-has contributed to improved insight into the social interaction between employees (Hildreth \& Kimble, 2004).

\subsection{What is a Community of Practice?}

CoPs play an increasingly pivotal role in knowledge management and the collaboration and networking activities in and between organisations. However, even though one definition is used more often than another, there is no generally accepted definition for a CoP. It is therefore desirable to formulate a definition that is in line with contemporary practice. Professionals increasingly learn in an informal manner in which social learning based on social interactions in communal environments plays an important role (Macia \& García, 2016). When speaking of communal environments, we often speak about CoPs. CoPs are often introduced as efficient and effective vehicles for knowledge management and are rapidly becoming the driving force of knowledge management programmes across the world (Wenger \& Snyder, 2000). Wenger (1998) introduced CoPs as an essential component in the social learning method of organisations and described the concept using three elements. First, members in a CoP are bound together by an informal agreement on a theme or topic, which is defined as a joint enterprise. A second element is mutual engagement: people in a CoP interact with each other and establish norms and relationships of mutuality that reflect these interactions. A third element that contributes to the formation of CoPs is the shared repertoire of communal resources such as language, routines, tools, stories, and practices that bind members together. A competent member has access to these repertoires and can use them appropriately.

The abovementioned description based on the three overarching characteristics (joint enterprise, mutuality and a shared repertoire) is a global description that forms the basis for many community development activities. However, in the past, several studies have been conducted to provide a short and appropriate definition for CoPs. The nature of these definitions often differs, and digital transformations, societal changes and developments in the business sector also cause possible changes in the concept of "communities of practice". It is therefore decided to conduct a literature review to analyse the definitions of CoPs used over a period of five years $(2014-2019)$ in order to come to an updated definition. An extensive review based on 60 articles that used a definition for a CoP has shown that in the majority of studies, the concept is defined by using one of the definitions of Etienne Wenger (Lave \& Wenger, 1991;Wenger \& Snyder, 2000;Wenger, 2004;Wenger, 2000). A total of 53 articles used for the review were collected by searching the Web of Science and Scopus databases using the term 'community of practice'. Collecting, processing and analysing the definitions has been carried out in a systematic way. 
I. Collecting: only the term 'community of practice' has been used in the collecting phase. Inclusion criteria were scientific articles published from 2014 to 2019 . For both scientific databases-Web of Science and Scopus - a filter on the relevance and the number of cited articles was applied. The number of citations gives an indication of the relevance of the article, while the most recent articles may use a more recent definition for a CoP.The first I 5 resulting articles of each search query were reviewed, making a total of 60 articles. Duplicate articles in the results were removed from the dataset $(x=7)$, which resulted in a total of 53 articles being evaluated.

2. Processing:Descriptions and definitions of a 'community of practice' were extracted from the articles and processed by applying text analysis to determine which (corresponding) terms were most prominent. Definitions used in multiple articles are included for each, as this is a valid indicator of the definitions' relevance. The articles in which CoPs are not defined are also excluded in this research project $(x=10)$. One article reviewed three definitions, and these three definitions were included in this analysis, bringing the total number of definitions to 45 . Overlapping terms were merged into one umbrella term (Table 2.I); for example, social networks comprise, among others, the terms 'social interaction' and 'social relationships'. In Appendix 2.I, all definitions used in this specific literature study are shown.

Table 2.I

Term frequency

\begin{tabular}{lc}
\hline Terms & x \\
\hline Common & 54 \\
Learning & 53 \\
Social network & 38 \\
People & 32 \\
Topic/Passion/Interest & 29 \\
Organisation & 27 \\
Group & 26 \\
Virtual/Web/Online & 9 \\
\hline
\end{tabular}
the analysis of the articles included in this research, we can conclude that many fellow researchers agree with the definition of Wenger, McDermott \& Snyder (2002), which is the most commonly cited definition used in this dataset as a basis for describing CoPs. The definition for a CoP reads as follows: "a group of people who share a concern, a set of problems, or a passion about a topic, and who deepen their understanding and knowledge of this area by interacting on an ongoing basis". This is a more specific definition that (partly) replaces one of the first definitions introduced by Lave \& Wenger (I99I): "Communities of Practice are groups of people sharing goals, activities, and knowledge in the context of a given practice". Since Wenger's definition is quite fluid and widely applicable - a practice can be, for example, a hobby, interest, or business situation-a more specific and demarcated definition is needed-at least for this research. Therefore, it has been decided to formulate a definition which is more appropriate in the current organisational context.

One of the fundamental structural changes in society since 2002 - when Wenger et al. (2002) wrote their respected article - that contributed to the decision to formulate a more adequate definition is the emergence of digital technologies. Before 2002, CoPs were often organized through physical activities where professionals held discussions, shared knowledge and/or collaborated. These activities often took the form of, for example, round table discussions, presentations, and small talk at the coffee machine or in corridors. The rapid expansion of information technologies (IT) and related digital communication applications more than a 
decade ago has brought people closer to each other and made the world smaller than ever. In addition, the expansion suddenly offered possibilities for the preservation and continuous improvement of knowledge. Knowledge became accessible to all colleagues instead of only the lucky ones who were invited and participated in meetings. This also had a positive effect on the transition from the primarily offline CoPs to (partly) online CoPs (Lee \& Neff, 2004). The advent of digital technologies and the internet has ensured that communal activities increasingly take place in online environments. This requires new skills and competencies from community members but also increases the media richness in the online modes of communication. Using ICT in learning environments offers possibilities for combining synchronous and asynchronous communication (Hlapanis \& Dimitracopoulou, 2007). Additionally, these technologies make it possible for community members to access knowledge stored in databases, have opportunities to come to know each other, engage in discussions and scale up the number of participants in these discussions (Hildreth \& Kimble, 2004). Kim, Hong, \& Suh (20I2) summarize the advantages of the increasing transformation of CoPs into virtual environments with the following four aspects: a high degree of collegiality, a generous shaping of time and resources, interactive and progressive problem solving, and a breakdown of previous geographical and hierarchical barriers.

Coming back to the definition introduced by Wenger et al. (2002), on the one hand this definition is specific in nature but, on the other hand, it is rather generic in its application. Currently, the majority of CoPs are introduced within the walls of an organisation (Pyrko, Dörfler, \& Eden, 2017), and increasingly, the initiation for a CoP is increasingly in the hands of an organisation's management (Lee \& Neff, 2004). In addition,Wenger's definition can be applied to virtually every community form because a practice for an individual can also take place outside the context of an organisation or knowledge domain.

To conclude, for this research, an updated definition for a CoP has been formulated: $A$ CoP is: "a social network where people in an organisational context come together around a common topic, passion or interest and regularly interact on- and offline with a focus on knowledge management, innovation, learning and social networking". These social networks may be used for empathetic support but are more often used for common interest, information sharing and problem solving. They are generally easy to enter and leave and non-exclusive and have a heterogeneous membership (Andrews, 2002; Lave \& Wenger, 199I; Wellman, 1997). There can be multiple sub-communities (groups) organized in one CoP that might include topics relevant to a diverse group of professionals. Typical topical examples of sub-communities are artificial intelligence applications by neurosurgeons, privacy measures for cloud computing in the financial sector or efficient and responsible use of eHealth as a preventive mechanism by caregivers.

\subsection{Community Infrastructure}

The online facilitated CoPs that are frequently implemented by organisations today require a suitable infrastructure (Barab, MaKinster, \& Scheckler, 2003; Jian \& Jaffres, 2006; Wenger, 1998). The infrastructure for CoPs within an organisation and between organisations is currently often supported by technologies but is not entirely dependent on these technologies. The CoPs in organisational networks use both online and offline events to enhance knowledge utilization (Mccully \& Lampe, 20II). Van Dijk (1997) and Dubé, Bourhis, \& Jacob (2005) emphasize that virtual communities will not replace organic, physical communities but will instead offer opportunities for each to strengthen the other. As defined by Dubé, Bourhis, \& Jacob (2005), 
a CoP is called "virtual" when its members use ICT as their primary mode of interaction. Being virtual does not preclude the use of face-to-face meetings, but several factors, including geographical dispersion and busy schedules, make communicating through ICT much more efficient. In this dissertation, the primary focus is on those events that exist in the online environment of a CoP.

Mcdermott \& Archibald (20II) and Gongla \& Rizzuto (200I) state that most communities should avoid using complex tools. Typically, most community members use only a few functions of such tools, such as a discussion forum, document libraries, expertise locators, on-demand teleconferencing or videoconferencing services, and online meeting spaces where members can edit documents as they discuss them. The researchers found that simplicity, ease of use, and familiarity are far more important than functional sophistication.

To achieve some uniformity in the technological composition of a CoP, Wenger, White, Smith, \& Rowe, (2005) distinguish between three functionalities that must be served. Their three functionalities also form the basis for the evaluation of the online community software used in the cases in this dissertation. The first functionality is the ability to support social interaction. This represents the possibility to discuss, brainstorm and ask questions. Huysman \&Wulf (2006) refer to the possibility of finding, communicating and cooperating with each other. In addition, these authors point out the option to share knowledge through network ties as an important functionality - the core of the second functionality that Wenger, White, Smith, \& Rowe (2005) mention: publishing. The CoP should offer options to publish content, for example by means of producing, sharing and collecting artefacts relevant to the practice. Regarding the supporting technology, most researchers refer to the ways to find, communicate, and cooperate with each other (Huysman \& Wulf, 2006).

Elaborating on the publishing functionality that forms a representation of the knowledge management activity, Huysman \&Wulf (2006) also introduced an additional characteristic of the generating functionality. This is the characteristic for selecting, editing, arranging, and searching data.The underlying technology for a CoP must offer optimal opportunities to create, share and search for knowledge and the expertise of others. The last functionality mentioned by Wenger, White, Smith, \& Rowe, (2005) is tending, which refers to a member's need for personal social behaviour and group behaviour. In the words of Bakardjieva \& Feenberg (2002), this functionality is the requirement to have options to interact on private and public levels and in open and closed groups. In addition, this functionality is represented in the members' need for personal social behaviour versus their willingness to cultivate the community (Pyrko et al., 2017). It is a matter of understanding what is happening, what the changing and emerging roles and positions are and how the structure evolves. These three categories for the technological functionalities form the basis for a community infrastructure definition: "The technological infrastructure of a community of practice is based upon optimal social interaction, publication, sharing and searching, and tending."

\subsection{What is Community Development?}

Starting a CoP is often part of an initiative from an organisation's management or of professionals on the work floor. This makes it clear that it is ideal when a CoP is a self-emerging or selforganizing network (Wenger, 2008). CoPs that have their origins at the management level are often called sponsored CoPs (Nickols, 2003; Ryan, 20I5), while the communities initiated by the professionals themselves are called self-organizing CoPs (Wenger \& Snyder, 2000). In this 
dissertation, we focus on the sponsored CoPs that exist in organisations. So, when further on in this dissertation the term community of practice or CoP is used, we refer to the sponsored versions of these CoPs. First, briefly, the differences between these two types of CoPs.

Sponsored CoPs — or formal CoPs — are initiated and supported by organisational management and are often expected to produce tangible, measurable and value-adding results that are beneficial for organisation. Sponsored CoPs are often supported at the business-unit level and are charged with specific goals such as creating new knowledge assets, identifying best practices and supporting expert consultations (Lee \& Neff, 2004). In the case of sponsored CoPs, organisations provide resources to community members but also assign formal roles and responsibilities to some individuals. These individuals often have a background in human resource management (HRM) or communication and therefore often have the expertise to stimulate interaction, networking, learning and knowledge management.

The self-organizing CoPs pursue the shared interests of the group's members. The initiative for these CoPs is in the hands of the community members themselves, but this also has the consequence that members come and go as the interests and issues shift and evolve. Wenger (1998) stresses the fact that community members develop practices that are their own response to external influences. Even when a community's actions conform to an external mandate, it remains the community - not the mandate- that produces the practice. In that sense, a CoP can always be considered as a self-organizing system.

Thus, the initiative for a CoP can be in the hands of an organisation's management or in the hands of the community members themselves. In the end, the development of CoPs is almost always the result of an organic system that emerges and is constituted and constantly reconstituted through interactions among the community members (Barab et al., 2003;Wenger, 1998). For CoPs to evolve and prosper and for all of the community members as well as the community organizers to benefit, the greatest challenge is to ensure that a balanced percentage of members in the community actively contribute to the community in various forms, such as asking questions, providing information and expertise, sharing ideas, and connecting with others (Wang \& Fesenmaier, 2004). The existing literature in the field of community development is situated in different domains and analysed from varying perspectives. Despite these different perspectives, there is mutual agreement that community development in all cases is stimulated by the ongoing provision of content that members perceive as valuable or useful (Koh, Kim, Butler \& Bock, 2000). The continuous delivery of new or updated content ensures that communities evolve from one stage to another.This development almost never runs smooth, but as McDermott (2000, p.6) stated decades ago, "A community's development, like an individual's is rarely smooth". Development is often not a linear process but an iterative process in which stages are skipped or repeated. The needs of community members and organisations evolve over time, which ensures that developments also differ.

One extensive study on community development was conducted by Matarrita-Cascante \& Brennan (20I2). These two authors define community development as "a process that entails organisation, facilitation, and action, which allows people to establish ways to create the community they want to live in. It is a process that provides vision, planning, direction, and coordinated action towards desired goals associated with the promotion of efforts aimed at improving the conditions in which local resources operate. As a result, community developers harness local economic, human, and physical resources to secure daily requirements and respond to changing needs and conditions" (Matarrita-Cascante \& Brennan, 2012, p297). This 
definition focuses on the construction of a highly fertile ground to reach the desired goals. However, community development is also often seen as a constellation of emerging events, a process from one milestone to another. The unpredictability of these events makes it difficult for community managers to create order amidst this chaos. In providing guidance and structure, several academics have created frameworks to describe and explain developments and possible interventions (a.o. McDermott, 2000). Multiple lifecycles and evolution models have been developed to identify and describe the stages that a community generally goes through (e.g., Gongla \& Rizzuto, 200I; McDermott, 2000; Sonnenbichler, 20I0). In the remainder of this section, the most common lifecycles and evolution models will be discussed.

Gongla \& Rizzuto (200I) observed the adoption, implementation and use of CoPs in IBM Global Services and discussed their evolution in terms of people and organisation behaviour, supporting processes, and enabling technology factors. Compared to other studies on community lifecycles, the work of Gongla \& Rizzuto (200I) has a focus on continuous development instead of on development with an end. This approach is - according to Nonaka \& Toyama (2003)—easy to explain because organisational knowledge creation is a never-ending process that upgrades itself continuously. The process of Gongla \& Rizzuto (200I) is exemplified by the following phases in order: a potential stage, a building stage, an engaged stage, an active stage and, finally, an adaptive stage.Wenger et al. (2002)identify the following comparable stages phases: potential, coalescing, maturing, stewardship, and transformation. Andrews (2002) suggests three stages: starting the online community, encouraging early online interaction, and moving to a selfsustained interactive environment. Malhotra, Gosain, \& Hars (1997)identified inception, initial user involvement, interactivity, and growth and experimentation activities as unique phases in a community. Iriberri \& Leroy (2009) based their research on the work done by Andrews (2002), Malhotra et al. (1997) and Wenger et al. (2002) and developed the following categorization: inception, creation, growth, maturity, and death. In the inception phase, a common need and vision is defined. When this common need and vision is available, a purpose is created, a technology is chosen, and an initial group of people is selected. This group of people-the ambassadors of a CoP_fulfils a stimulating role towards future participants (Young, 20I3). The extent to which they are successful in fulfilling this role influences the subsequent phase. This might be a maturity phase in which the commitment of community members and their trust in others lead to the development of new subgroups and new relations, but it can also a phase in which there is a sign that the community will die a silent death.

Gongla \& Rizzuto (200I) also point out that communities may stay at certain stages and not evolve to another level, communities may move "backward and forward" between the stages, communities may have some characteristics of one stage while they are still primarily at another stage, and communities may "rest" for extended periods at one stage and suddenly evolve quickly to another stage. Looking at these patterns, it becomes possible to determine and predict the general stage a community is in, but specifically identifying or determining when and how a community evolves is often a hard task for community managers. This is especially due to the rapid changes that occur in these communities. Throughout the life of the community, new members join in and old members whose needs are satisfied or whose initial excitement for joining the community has worn down leave the community. As new members join, the community evolves and a cycle of interaction repeats (Iriberri \& Leroy, 2009; Preece, Nonnecke, \& Andrews, 2004; Ridings, Gefen, \& Arinze, 2006).

In this research, we approach CoP development as a continuous process in which collaborations are organized, substantive or organisational knowledge is developed and social (professional) 
networks are built and maintained. As soon as an organisation-in the case of a sponsored CoP-pulls the plug from a CoP, it marks the end of the community. Community members in self-organizing CoPs may jointly acknowledge that a CoP no longer adds value to their individual and collective values and another knowledge development approach must be initiated. To prevent a CoP from dying silently or going in an unwanted direction, different roles and types of community members are often identified and/or assigned. Community members, for example, focus on gaining more insight into the developments in their CoP to stimulate the community to go in a preferred direction.

\subsection{What are Community Member Types?}

Ultimately, the main objective in each CoP is to attract many community members to actively participate and develop lasting relationships (Iriberri \& Leroy, 2009), which requires an active enabler or group of enablers and strategic community management (Wenger, McDermott \& Snyder, 2002). Studies on community member types focus on the roles that individual community members take or on the positions that community members take. The community member role is a role that a community member takes by interacting with the group in a certain way. The community member position is determined by a network of interactions in which a community member is involved, and which determines his or her relative network position in a CoP. In the remainder of this section, a more detailed description of these two perspectives is given.

\subsection{Community Member Roles}

CoPs in work contexts often accommodate participants who fulfil a role chosen by the organisation and participants who fulfil an informal, self-chosen role. Especially in the first stages of a community's life cycle, a community member receives a certain role from his or her organisation, such as community manager, ambassador or coordinator (Jahnke, 20I0). Understanding a community's life cycle stage and the types and intensity of interactions during these phases is fundamental for community managers to act in accordance with what is expected. The members with a formal, activating role are extremely important for the success of the community (Young, 20I3). These enablers are needed to stimulate social interactivity, invite relevant people and connect members.

Informal roles are often taken based on expertise, experience or circumstances. In contrast to the formal roles, these informal roles are not assigned by a contract nor triggered by any rules but are observable via interaction patterns and activities in the community (Jahnke, 20I0). In defining the different community roles, the first discussion is often when individuals can call themselves community members. Bryant, Forte, \& Bruckman (2005) tried to formalize this by suggesting that participants become community members when they move from the less active, peripheral part of a community to the centre of the community by giving more input. However, questions remain about when a participant is a participant in the peripheral regions of a community and what the crucial moment is for becoming an actual community member. Is a participant who is reading or viewing knowledge material in the community a person in the peripheral region of the community, in the core of the community or in one of the shells around the community? If we have layers between the periphery and the centre of the community, what are these layers?

By reviewing the existing literature around community member roles, it becomes clear that there are different layers between the peripheral group and the core group. For example, 
Borzillo, Aznar, \& Schmitt (20II) identified three levels of social behaviour: a core level, an active level and a peripheral level. The way community participants move through a community largely differs by case. A CoP often makes a flying start in the first couple of months in which community members participate out of enthusiasm or curiosity, the 'novelty fatigue' (Feenberg \& Bakardjieva, 2004). Wenger (2006) compares the enthusiasm of these few participants with the start of a relationship: "It has the same mixture of excitement and apprehension, the relationship is palpable; it feeds the imagination, but the prospects remain unclear". In addition to this limited group of enthusiasts, the vast majority of CoP members consist of participants who can be characterized by their legitimate peripheral form of social behaviour. Lave \&Wenger (1991) describe this form of social behaviour as the process where newcomers start in the peripheral area of a CoP and move-with changing paces - to becoming more experienced members, eventually becoming old timers. In peripheral activities, novices become acquainted with the tasks, vocabulary, and organizing principles of the community. This group, which forms a substantial part of the whole community, develops in different directions, resulting in a variety of member types. It largely depends on an author's research perspective on how the roles or member types are identified and defined, but in general, three recurring member types with varying titles can be identified: the community leader, the contributor and the information absorber (Drew, McCallum, \& Roggenhofer, 2016). Many CoPs start with a formally assigned community leader, and this leader intends to stimulate others to become active community members. The resulting active group can be defined as the contributors; this group shares their knowledge, experiences and best practices. This group can be divided into several other types of contributors, for example, into posters (Drew et al., 2016), commenters (Miller \& Proctor, 2016), chatterers (Purvis, Sambamurthy, \& Zmud, 200I), advisers (Wellman, 1997) and appreciators (Bista, Nepal, \& Paris, 2012).

This subdivision of community member roles is not exhaustive. Especially when analysing the type and intensity of individual social behaviour, it is obvious that, for example, regarding the role of posters in a community, a further specification is possible for the poster role. Jahnke (2010), for example, separated the role of a 'poster' into authors, scaffolders, visitors, conflict mediators, technical supporters, conclusion makers, procedure promotors, organisational supporters and decision initiators. With text analysis, one can identify these different 'posting' roles. In general, identifying individual community roles is fundamental for controlling the success of CoPs. The changing roles of individuals can have a high influence on the development of the community. In addition, the changing roles can act as triggers to evoke community changes. A typical example of the effect of these roles changing can be seen when a community manager leaves the community, which might cause the remaining community members to become less active or become inactive.

\subsubsection{Community Member Positions}

The individual position of an actor-an individual community member-is closely related to the function of that node in the CoP. The position of the community members within social networks has an effect on their social outcomes, such as their creativity, innovation capacity, knowledge distribution, promotion opportunities, social support, and productivity (Safadi \& Faraj, 20I4). In social capital theory, an individual's network position is determined by his or her relationships with others in a social system (Brass, 1984). A well-known study by Granovetter (1973) shows that not only the strong ties are fundamental for building a mutual understanding of expertise and for achieving better performance but also the weak ties among actors are an important stimulus for reaching opportunities beyond the local communities that are normally 
not accessible via strong ties. Combining the strong ties with the weak ties can, according to Safadi \& Faraj (20I4), have a great influence on reaching the optimal use of the complementary resources.

Persons who are more central have many direct connections, have an important role in activating others, and are seen as the leaders of the community (Brass, 1984; Johnson, Safadi, \& Faraj, 20I5). The centrality of an individual is often measured by calculating the degree centrality, which reflects the number of relationships a person has. Freeman (1978) was one of the first academics to create a measurement to determine the position of individual users in a network based on centrality scores. It is often expected that community members with a high degree centrality are more active, prestigious, powerful and visible than other, more marginal community members, particularly due to the many ties and connections the former have to other community members in the network (Freeman, 1978). However, the central position of these individuals makes an organisation often quite vulnerable if these people leave the organisation.

Two of the other well-known measures developed by Freeman (1978) are the betweenness centrality score and the closeness centrality score. The betweenness centrality score refers to where an individual fall between pairs of other individuals along the shortest path connecting them. This implies that the position of the individual with the highest betweenness centrality score has potential control over others. Kratzer, Lettl, Franke, \& Gloor (20I6) uses the term 'lead users' when referring to people with a high betweenness centrality score. Kimmerle et al. (20I3) recognize a 'mediating' position for these community members due to their ability to filter the knowledge-exchange channels inside the community. In a CoP, this implies that the experienced members who are supposed to act as mediators control the processes of knowledge development and practice improvement. They have a large influence on the transfer of items through the network, under the assumption that item transfer follows the shortest paths. Therefore, these network members are often seen as brokers, bridges, lead users or boundary spanners (Kratzer et al., 20l6). These individuals position themselves between different clusters in a network and are critical to gathering and transferring information.

The closeness centrality score is generally calculated by summing the length of the shortest paths from one community member to all other community members. Freeman (1979) argues that the closeness measure shows the independence (the extent to which an actor can avoid the control of others) or efficiency (the shortest number of steps required for an actor to reach all other actors) of individuals. A central community member-measured by closeness centrality-can reach other community members in a minimal number of intermediary steps and is therefore dependent on fewer intermediary positions than the community members in the peripheral regions of a community. It is assumed that people with a high closeness centrality score have or take a more leading role within a network (Sonnenbichler, 2010), since these individuals have the opportunity to reach a large group of people.

Community members in the peripheral regions of a community are often seen as observers with a minimal amount of contributing value (Sun, Rau, \& Ma, 20I4). However, the fact that these people are at the borders of the community has the consequence that these people are also less embedded in the organisation and are better positioned to benefit from divergent ideas developed through relationships with external actors (Dahlander \& Frederiksen, 20I2). In addition, a substantial number of these members are also able to span boundaries between sub-communities or between different communities and to bring fresh ideas and solutions to 
problems of the central members (Jeppesen, Lakhani, \& Jeppesen, 20I0). In this dissertation, we use the subdivision of centrally located persons, bridging persons and leading persons when the network positions are being examined.

\subsection{What is a Community Foundation Framework?}

The purpose of the community foundation framework is to make a clear distinction between the cases in this study, based on organisational objectives, implementation strategies, and organisational cultures. In the coming paragraphs, an explanation is given of these concepts.

\subsection{Organisational Objectives}

Today's contemporary work contexts continue to increase in complexity (Schneckenberg, 2009). As a result, employees in organisations increasingly become experts in a certain domain instead of in multiple domains. A brain drain is therefore the nightmare that occurs in an organisation when employees with specific and unique knowledge leave the company. To cope with this kind of issue, organisations increasingly start initiatives to empower employees to collaborate, share explicit knowledge and concretize tacit knowledge ( $\mathrm{Li}$ et al., 2009). These types of social behaviours that are facilitated in CoPs are the main reason for organisations to start these initiatives. Online community software licences are bought to facilitate and support these actions, also called the development of CoPs. To monitor and control developments, many organisations attempt to formulate objectives that contribute to individual, collective and organisational value development.

The objectives that organisations formulate are often more specific versions of types of social behaviour, as described by Li et al. (2009). Lesser and Storck (200I) offer a more specific form of objectives for organisations, for example, to "share experience and expertise across similar projects", "develop and exchange implementation and training techniques", "share knowledge about a new industry development" or "transfer experience and techniques across industry groups". These examples are rather context-sensitive and therefore not always applicable to situations in other CoPs.

The CoP literature shows that most organisations initiating a CoP organize it as an essential component of their knowledge management strategy alongside mentoring, staff development, knowledge capture, and knowledge sharing (Curran \& Murphy, 2009). Annabi, Mcgann, Pels, \& Arnold (2012) studied the possibility of aligning business objectives with CoPs and generated seven unique business objectives corresponding to the possibilities that CoPs offer. These objectives are as follows: knowledge management, increases in productivity \& innovation, customer relationship management, collaborative content creation, increases in internal crossfunctional collaboration, knowledge retention and training, and improvements in decision making and productivity.

Nickols (2003) introduced another categorization to identify the objectives that can be fulfilled by introducing CoPs, and more or less comparable organisational objectives are also introduced in other studies, such as those of Du Plessis (2008) and Hoadley (2012). These objectives are focused on enabling collective knowledge exchange, enabling individual and collective learning by sharing relevant content, and creating measurable and tangible value-adding benefits for businesses. The last is also often operationalized as the developing and sharing practices that will contribute to lower costs and an increase in the revenues for an organisation once 
the practices have been deployed and replicated across the organisation (Probst \& Borzillo, 2008). Previous studies have already shown different perspectives on defining organisational objectives. It becomes clear that each organisation formulates its objectives more specifically or less specifically according to its expectations. Loyarte \& Rivera (2007) extensively reviewed the literature around the cultivation of CoPs in organisations. They state that when organizing CoPs, it is important to align the CoPs well with the personal objectives of professionals as well as with those of the organisation. The objectives set by organisations when initiating a CoP are defined as follows: increasing the number of customers, reducing costs, preventing brain drains, stimulating organisational learning, making optimum use of the innovative capacities in the organisation, changing organisational culture and generating value.

Reflecting on the categorizations made by Li et al. (2009), Annabi et al. (2012), Curran \& Murphy (2009), du Plessis, (2008), Hoadley (20I2), Lesser and Storck (200I), Loyarte \& Rivera (2007), Nickols (2003) and Probst \& Borzillo (2008), we see that five overarching categories can be identified to define the organisational objectives when initiating CoPs. These objectives include stimulating collective learning, innovating and improving practice, making knowledge management more efficient, creating tangible, measurable and value-adding benefits for the organisation, and increasing opportunities for professional networking.A good understanding of the objectives that organisations have with a CoP seems crucial for monitoring and evaluating developments within the CoPs.

\subsubsection{Implementation Strategy}

The implementation strategy has an important role in determining the actual direction that a CoP is going. When speaking about implementation, we use the definition introduced by Bouwman, Van den Hooff, Van de Wijngaert \& Van Dijk (2005): 'the phase of internal strategy formation, project definitions and activities in which the adopted application is introduced within the organisation, with the aim of removing reservations and stimulating the optimum use of the application'. Several frameworks have been developed in recent decades to support implementation processes in practice. Typical examples of moments when implementation strategies play an important role is with the introduction of new technologies (Bouwman, Van den Hooff, Van de Wijngaert, Van Dijk, 2005), new methodologies (Drohomeretski, Gouvea Da Costa, Pinheiro De Lima, \& Garbuio, 20I4), and new policies (Van Metre \& Van Horn, 1975).

A framework of Van der Zee (1995) included in the book of Bouwman, Van den Hooff, Van de Wijngaert \& Van Dijk (2005) distinguishes four implementation strategies: the diffusion approach, the directive approach, the interactive approach, and the development approach. Each of these strategies differs in its approach to convincing employees of the necessity and/or added value of innovations. In the diffusion approach, the innovation sells itself; therefore, employees are inclined to adopt and use the innovation for their own practice. The focus in the directive approach is more on forcing employees to use a new technology or innovation; the decision and implementation strategy are organized in a top-down manner, and the preferred end situation for management is clear. In the case of the interactive approach, professionals decide for themselves how they will use the new technology or innovation. The last approach, the development approach, is based on increasing the competences of the organisational members to make them capable of using the technology or innovation.

The defined strategies are formulated for the purpose of implementing technological innovations in organisations. Innovations change the work within the organisation but also serve 
a purpose that goes beyond the type of technology, for example, working with more agility or improving knowledge exchange. Technology is a means for realizing this behavioural change, but implementing a new technology should not be an end in itself (Kane, Palmer, Philips Nguyen, Kiron, \& Buckley, 20I5). In practice, these approaches are not used in their pure form but often used in a more hybrid form. Implementing a new concept is often a complex activity in which many disciplines within an organisation must be involved. For example, before implementing a new technology in an organisation, it is also crucial to have that technology implemented in existing technological systems to ensure that it reduces the complexity for the end-users. The fragmentation caused by using different IT systems within an organisation not only leads to a lack of data interchangeability (organisational relevance) but also to confusion among users and a technological overload by these users (individual relevance) and ultimately it also leads to a lack of efficiency and effectiveness, as data have to be entered in different places. In this dissertation, the diffusion approach, directive approach, interactive approach and development approach are used to understand the implementation strategies of the organisations in this dissertation. However, when stimulating widespread implementation of new innovations, it is important to address contextual factors as well (Quinn, Cook, \& Rowland, 2019). An example of a factor which is important there is the topic in the next paragraph: organisational culture.

\subsubsection{Organisational Culture}

Academic research indicates that organisational culture affects the social behaviour of individuals within knowledge-intensive and creative activities (Schepers \& Van den Berg, 2007). The knowledge-based view of firms suggests that intellectual resources are key organisational assets that enable a sustainable competitive advantage and more effective and efficient work. The context in which individuals create, store, share and use knowledge has a significant role in these KM practices (Alavi, Kayworth, \& Leidner, 2004). Schein (1985) describes the organisation culture at three levels: basic assumptions, values, and artefacts. The basic assumptions represent interpretive schemes that people use to perceive situations and to make sense of ongoing events, activities, and human relationships, thereby forming the basis for collective action (Van Maanen \& Barley, 1984). Values can be seen as a set of social norms that define the rules or context for social interaction through which people act and communicate (DeLong \& Fahey, 2000). The artefacts may include things such as art, technology, and visible and audible behaviour patterns as well as myths, heroes, languages, rituals and ceremonies (Pettigrew, 1979). In this research, we have chosen to conceptualize organisational culture in terms of 'values'. The main reason for this is the fact that values are more easily studied than basic assumptions and artefacts. However, most of the studies conducted in the past have focused on value-based theories of culture, especially in the context of KM and collaboration.Alavi et al., (2006) suggest that it is questionable that an organisation has only a homogeneous culture; they propose that an organisation consists of one homogeneous culture with multiple local cultures. However, this largely depends on the size of the organisation; it is likely that smaller organisations regularly have a more homogeneous organisational culture than larger organisations.

As discussed, the culture within an organisation largely determines how practitioners behave. Typical cultural sources that affect the structuring in organisations are education, experience, and interaction styles of the people within that organisation (Schwieger, Melcher, Ranganathan, \& Wen, 2004). Academic research on organisational culture has largely become saturated in recent years. One of the commonly used frameworks for examining organisational cultures is the competing value framework (CVF, Quinn \& Rohrbaugh, 1983). CVF consists of two dimensions: "internal versus external orientation" and "stability versus flexibility". An internal 
focus reflects care for people and efficiency, whereas an external focus echoes the level of awareness of the organisational environment. The stability and flexibility on the other axis represent the difference between an orientation towards top-down control, the application of formal rules and decisions, and a focus on adaptation and change (Schepers \& Van den Berg, 2007).

In the quadrants of the resulting matrix, four organisational cultures can be identified: clan, adhocracy, hierarchy and market. Each of these perspectives on organisational cultures has different assumptions about sharing knowledge and about collaboration within knowledge domains, which leads to variations in the influences on the use and outcomes of group behaviour

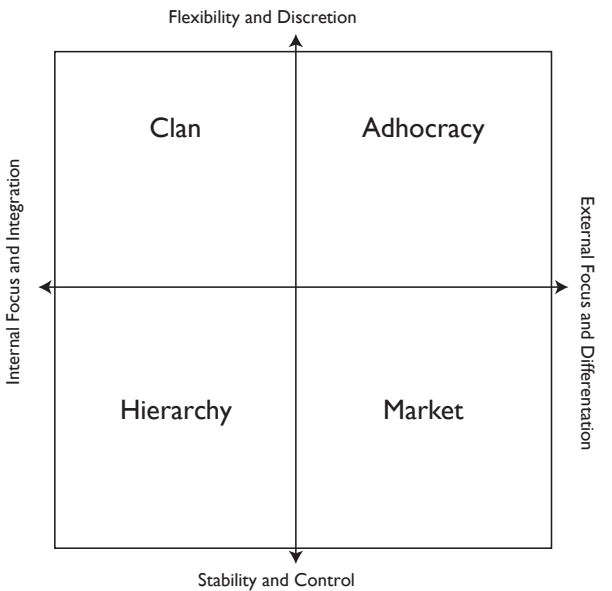

Figure 2. I. Competing Values Framework (Quinn \& Rohrbaugh, 1983)

Organisations with a clan culture are marked by a focus on people and sharing between individuals. This organisational culture concentrates on teamwork, loyalty, and commitment (Fong \& Kwok, 2009). Adhocracy culture is dynamic and creative and has little formalization of roles and responsibilities (Vries, 2003). In this culture, risk taking to create new innovations is stimulated, which results in the encouragement of employee initiative. More hierarchical cultures are characterized by formal structures that focus on maintenance and a need for stability and control. The focus in such cultures is on working on practical cases in a structured way. Organisations with a hierarchical culture "stress documentation, information management, stability, and control” (Schepers \& Van den Berg, 2007, p. 4II). The last culture is market culture, which describes an open organisation with a focus on both internal and external positioning and a need for stability and control. This culture is often perceived as a results-oriented culture with a customer-based culture (Schneider, Ehrhart, \& Macey, 20I3). It focuses on organisational effectiveness and operates as a market. 



\section{3 \\ Community of Practice Development Model}

\section{I. Introduction}

The previous chapter defined the terminology relevant to this research project This chapter addresses a substantial part of the literature around models and underlying factors that affect individual and group behaviour (e.g. knowledge management, collaboration, social networking) in communal environments such as CoPs, online communities, communities of interest et cetera. Most academic studies generalize knowledge management (KM) in organisations as the exchange of knowledge, but ignore other important steps within the KM, such as network development, consultation, and discussion. These factors encourage the development and sharing of knowledge. Therefore, this chapter attempts to answer the first sub-question in this dissertation by focusing more on the processes within online KM environments:

Which factors potentially affect the social behaviour of community members in communities of practice?

Some typical sociological, psychological, and communicational theories are reviewed regarding the composition and development of social interaction and group behaviour and therefore form the basis for answering the first sub-question.

Humans are social animals. Beliefs, desires, and behaviours are affected by our social preferences, our relationships, and the social contexts in which we live and make decisions. We are "groupminded individuals" who see the world from a social as well as an individual perspective; we understand what is in the minds of others and often act as if our brains are networked with the brains of other people (Goldie, 2016; Tomasello, 20I4). Social interaction seems to be one of the most important human needs. It is not without reason that social interaction enlarges the knowledge level of individuals (Chiu, Hsu, \& Wang, 2006). Individual learning and new knowledge creation occur when people combine and exchange their personal knowledge with others (Wasko \& Faraj, 2005). However, field studies in diverse settings indicate that employees frequently resist sharing their knowledge with the rest of their organisation (Barragán-Ocaña, Quijano-Solís, Vega-Díaz, \& Sánchez-Lara, 2012). Ardichvili, Page, \& Wentling (2003) identify two types of barriers for individual and group behaviour in a CoP. The first group of barriers relates to an individual's perception that existing tight-knit, face-to-face groups already fulfil their knowledge need and the second group of barriers dwells in the context-dependency of issues. Individuals feel that the solution for a problem can often not be duplicated in their own situation.

Nevertheless, an increasing number of professionals and organisational management personnel recognize the added value of collaborative or social learning (Argote, 2012; Qi \& Chau, 2018). Therefore, CoPs have been introduced into organisations. These CoPs are contexts for organisational learning and continuing professional development, in which members acquire knowledge through their social behaviour in mutually beneficial social interaction. Over the last decade, many researchers have tried to define an optimal form of social behaviour, but due to 
the considerable amount of disagreement among these researchers there is no consensus on what this optimal form must be. In this dissertation the Motivation, Opportunity, and Ability model (MOA model) (Macinnis et al., 1991) is the starting point for defining critical factors for individual and group behaviour in a CoP.This model has specifically been designed to provide a framework for describing the factors that influence and explain behaviour in various contexts, and is often used in relation to the concept of knowledge management (Fadel \& Durcikova, 2014; Kettinger, Li, Davis, \& Kettinger, 2015; Siemsen et al., 2008). An individual's actions or performance are the result of the willingness and motives of professionals (motivation), the chances they have to perform (opportunity), and their capacities as professionals (ability). Based on the scientific literature, the model has been extended and improved to explain individual and group behaviour more accurately. In the upcoming paragraphs, the MOA model is introduced and in the last few paragraphs we elaborate upon the model with some additional concepts related to the contextual focus that are supposed to affect group behaviour.

\subsection{Social Behavioural Theories}

In the range of personal motivation theories and social behavioural theories a couple of respected theories are often used, including the following: the theory of planned behaviour (TPB) (Ajzen, 1985); the theory of reasoned action (TRA) (Fishbein \& Ajzen, 1975); the motivation, opportunity, ability theory (MOA) (Macinnis et al., I99I); but also the uses and gratifications theory (UGT) (Katz, Blumler \& Gurevitch, 1973); the technology acceptance model (TAM) (Venkatesh, 2000); and the unified theory of acceptance and use of technology (UTAUT) (Venkatesh, Morris, Davis, \& Davis, 2003).

In the identification of the most appropriate social behavioural theories in the context of this study, criteria were used to evaluate the models. The origin of these factors is based on both theory and situational factors that are specifically relevant to the context of this research project. The first criterion is attention to the context in which the CoP is applied ('contextspecific enablers'). Knowledge-creating processes are - according to Nonaka \& Toyama (2003) - necessarily context-specific, as it is the context that determines the direction of social behaviour. The second criterion is the opportunities for the application of the theory in comparable community - and knowledge management situations ('comparable situations'). The third criterion is the accuracy and simplicity of the theory, as it must be a accurate model that is precise but simple (Weick, 1979). Practitioners (e.g. organisations and their employees) can easily use the results to adapt organisational policies and improve professional support in order to optimize the CoPs ('accuracy and simplicity'), and the last criterion is the focus on actual behaviour instead of on intentions ('actual behaviour'). The data gathering for the study was conducted in real-life situations where community members actually behave in a certain manner.

In Table 3.I, an overview is given as to what extent these theories are appropriate for the objectives in this dissertation. The decision to use the MOA model as an underlying framework instead of the TPB,TRA, and UTAUT is based on the extent to which the theory meets the set of criteria summarized in Table 3.I. 
Table 3.I

Appropriateness of the theories

\begin{tabular}{|c|c|c|c|c|}
\hline Theory & $\begin{array}{l}\text { Context-spe- } \\
\text { cific enablers }\end{array}$ & $\begin{array}{l}\text { Comparable } \\
\text { situations }\end{array}$ & $\begin{array}{l}\text { Accuracy and } \\
\text { simplicity }\end{array}$ & $\begin{array}{l}\text { Actual behav- } \\
\text { iour }\end{array}$ \\
\hline TPB & $x$ & $x$ & $x$ & - \\
\hline TRA & - & - & $x$ & - \\
\hline MOA & $x$ & $x$ & $x$ & $x$ \\
\hline UGT & - & $x$ & - & $x$ \\
\hline TAM & - & $x$ & - & - \\
\hline UTAUT & $x$ & $x$ & - & - \\
\hline
\end{tabular}

The MOA model takes other factors into consideration that might affect an individual's behaviour, such as the individual's motivations, capacities and opportunities. Another criterion for the sake of this research project is the focus on intention as a predictor for actual behaviour. TRA and TPB - for example - start from the supposition that the intentions of the individual largely reflect his or her personal attitudes. As an example,TRA uses the individual's attitude towards a certain behaviour, the prevailing subjective norms and the perceived behavioural control of the individual towards a certain behaviour as a predictor for the individual's intention. In that case it remains debatable whether this ultimately also has an impact on the actual behaviour. In this research project, individuals show actual behaviour and the MOA model aims to explain that actual behaviour. The factors described in both the TRA and TPB form an important resource for the MOA and therefore also form an important underlying framework for the research model used in this research project.As an example of the similarities between the TRA/TPB and the MOA, the following comparison can be used: attitude towards behaviour partly represents the motivation, subjective norms can also be a motivation to participate, and the perceived behavioural control is comparable to an individual's ability or capacity. A substantial difference between the MOA and the TRA/TPB is the fact that the TRA and TPB both focus on the control over his or her own behaviour, while the MOA takes the external stimuli (opportunities) as an important resource for actual behaviour. In the case of CoPs this implies that organisations have an important function in creating the preconditions for exhibiting the desired behaviour.

In this exploratory research project, the MOA is used as a conceptual framework to explain individual and group behaviour. The decision was made to integrate some critical components from the TRA, TPB, UTAUT and other relevant theories in the MOA model to create an integrated framework to explore individual and group behaviour in CoPs. These added components, which are not specifically covered in the original MOA model, will be outlined in the upcoming paragraphs. These paragraphs provide an explanation of the underlying variables, which-it is assumed-are important indicators of motivation, opportunity and ability. This is followed by an explanation for the further specification of the conceptual model in this dissertation. 


\section{Motivation, Opportunity, and Ability Model}

The Motivation, Opportunity, and Ability model (MOA model) is a model-initially developed for consumer research - that is based on the premise that consumers experience barriers to action because they lack either the motivation to act, the opportunity to act, the ability to act, or a combination of the three factors (Maclnnis \& Jaworski, 1989). However, in the last few years, the MOA model has also been increasingly applied to community-based settings (Fadel \& Durcikova, 20I4; Gruen \& Czaplewski, 2005). (Fadel \& Durcikova, 20 I4; Gruen \& Czaplewski, 2005). Siemsen, Roth, \& Balasubramanian (2008) posit that this customer-centred theory is also useful in the context of employee communication. In their study, the theory is used to describe the behaviour of employees as a function of their motivation, opportunity and ability. As discussed by Hung \& Petrick (20I I), all MOA-related studies have been conducted to explain information processing behaviour or a decision-making process.

\subsection{Motivation(s)}

Individuals' motivation is seen as an important internal driving-force in the decision-making process of action, as it affects both the direction and the intensity of individual behaviour (Bettman, 1979; Macinnis et al., 199I) It is more likely that community members participate when the subject becomes relevant on a personal level. Bayton (1958, p. 282) - one of the pioneers in research on motivation - defines motivation as "the drives, wishes, urges or desires which initiate the sequence of events known as behaviour". Maclnnis \& Jaworski, (1989) draw attention to the fact that motivation does not automatically lead to actual behaviour, and thus they adopt the point of view of (Park \& Mittal, 1985), whose more limiting definition of motivation is "goal-directed arousal".

However, it is essential to understand the two different measures to study motivation. Motivation can be measured by its level and by its type.

\section{Level of motivation}

The original MOA model (Macinnis et al., I99I, p. 34) restricts motivation to "a consumer's desire or readiness to process brand information in an ad". Other related terms often used are willingness and interest in showing certain behaviour (Gruen \& Czaplewski, 2005). Higher motivation in that case implies that people have a higher willingness to process information in for example an advertisement or an article. In the context of CoPs, motivation is defined as a community member's interest in engaging in activities with other community members. Siemsen et al. (2008) and Reinholt, Pedersen, \& Foss (201 I) used the actual behaviour perspective and found that motivation directly affects knowledge sharing in CoPs. According to the social exchange theory, knowledge sharing is highly related to an individual's strong individual motivation (Kankanhalli, Tan, \& Wei, 2005). Minimally motivated participants are less likely to show social behaviour that somehow matches the expectations within CoPs that members will socially interact with other community members. As Nahapiet \& Ghosal (1998) and Sukoco \& $\mathrm{Wu}(2010)$ state, the motivated member will be energized, interested, and willing to engage in community activities. However, to create a comprehensive picture of the concept of motivation, this study investigates community members' motivation from a multidimensional perspective, as suggested in the work of Sukoco \& Wu (2010).

Type of motivation

Bigné, Ruiz, Andreu, \& Hernandez (2015) refer to the operationalization of motivation in the MOA model as a weakness."One of the most important weaknesses of MOA studies is that they 
do not take into account the antecedents of overall motivation" (p. $2 \mathrm{II}$ ). When the research goal is to provide a more focused explanation for certain individual behaviour, extending the motivation with antecedents for motivation becomes an important step. Numerous individual motivations are possible to determine whether one should participate in a CoP, these can be different for each individual. In this section, we therefore list a select number of individual types of motivation that are frequently related to individual and group behaviour in (online) communities (of practice)

The first one is related to the principle behind a CoP: improving a certain practice. The idea behind a CoP is to optimize existing practices and create new practices in organisations. Correia, Paulos, \& Mesquita (2009) suggests a potential motivation that specifically explains individual and group behaviour in a virtual CoP.According to these authors, contributing jointly to the success of the organisation is an important motivation for displaying individual and group behaviour in a CoP. Components such as socializing (Lee \& Ma, 20I2) and altruism (Bettiga, Lamberti, \& Noci, 2018) can be categorized under this collective motivation to improve work performance. Following the results from a study of Lee \& Ma (20I2) leads to another important motive for active forms of individual behaviour: status seeking. Bettiga, Lamberti, \& Noci (2018) refer to altruism and social reputation as motivations for pro-active social behaviour within CoPs. Improving social reputation - or seeking for status - is more often found as a significant predictor of social behaviour in (online) communities (Lampel \& Bhalla, 2007).

Most studies that have used motivational factors in the context of describing or predicting social behaviour, particularly in knowledge management, focus on the active types of social interaction behaviour: creating and sharing knowledge (Hung, Durcikova, Lai, \& Lin, 20I I; Siemsen et al., 2008). Using such a perspective assumes that everyone has the intention to exhibit proactive social behaviour-a situation that does not necessarily apply to CoPs, where the majority of participants primarily acquire knowledge and information rather than share it. Lee \& Ma (20I2) refer to the desire to stay informed as an important motivation for an individual's social behaviour in virtual environments. A motivation which is strongly associated with knowledge acquisition, but also with knowledge sharing behaviour.

The idealistic image that sometimes lives in CoPs arises from a collective desire to improve a certain practice or knowledge management in an organisation; these desires do not count as an important motivator for every participant. Some studies have also shown that the subjective norm —or social pressure — stimulates an individual's social behaviour in a CoP (Chen, Chen, \& Kinshuk, 2009). Hung, Lai \& Chou (2015) found evidence that the subjective norm affects both an individual's knowledge sharing behaviour and an individual's knowledge acquisition behaviour. In this, the subjective norm refers to others' expectations about a person's behaviour (Ajzen, I99I) and is strongly determined, according to Ham, Jeger, \& Ivković (20I5) by the perceived social pressure from others such as colleagues and management and what affects their way of behaving.

Many academic researchers have studied the motivations of individuals to participate in communities (Chang \& Chuang, 20II; Thakadu, Irani, \& Telg, 20I3; Wasko \& Faraj, 2005). Substantive reflection on these motivations results in a categorization of four motivations that apply to social behaviour in CoPs. These four categories are the motivation to be informed, the motivation to improve one's work performance, motivation related to improving one's social reputation, and subjective norms. 


\subsubsection{Opportunity}

Due to the current economic situation, internationalization and increased competition, the pressure on organisations is increasing. This results in increased time pressure on practitioners. More often, practitioners need to perform extra activities and tasks within the same time as before due to ongoing changes in their organisations or changes in regulations. To be able to better organize work, many organisations therefore initiate means to improve cooperation and knowledge exchange within - and also increasingly between-organisations. CoPs are one of the means that contribute to this ambition. However, all of the restrictions and external barriers reduce the chances of practitioners participating in CoPs. When organisations legitimize social behaviour, the chances of the success of the CoPs will rise:"Organisations can support CoPs by recognizing the work of sustaining them; by giving members the time to participate in activities, and by creating an environment in which the value communities bring is acknowledged" (Wenger, 1998, p.7).

To stimulate certain behaviour in organisations, management in organisations must recognize and support this behaviour. Providing a fertile basis to show this behaviour is often a first step. Three of the fundamental terrains in which these opportunities can be created are time (Orlikowski, 1993), work autonomy (Cardona, Lawrence \& Bentler, 2003; Salanova, Peiró \& Schaufeli, 2002) and facilitating conditions (Bhattacherjee, 200I; Fadel \& Durcikova, 20l4; Venkatesh et al., 2003). Organisations who give employees time to participate in CoPs show their employees the relevance that they attach to the CoP. If the management of an organisation expects its employees to participate outside of work time, then an employee can perceive this as an important indicator of the value that the organisation places on their social behaviour. The actual provision of time to promote knowledge management and cooperation within CoPs is therefore an important indicator of the perceived relevance of the CoP for the organisation. Time is, according to Siemsen et al. (2008), seen as an important operational constraint among colleagues on participating in a CoP.The degree to which an employee has a margin of manoeuvre during a working day to do more than just the regular activities determines employee's social behaviour. Another important precondition that an organisation can optimize is encouraging autonomy in individual work so that professionals themselves are free to engage in what they consider to be relevant behaviour (Annabi et al., 20I2). If a professional has more autonomy to choose when, where, and how to share knowledge, the professional will feel more responsible for his or her work outcomes (Cabrera, Collins, \& Salgado, 2006). A last important means of support that the organisation can offer are the facilitating conditions. This factor-developed in the unified theory of acceptance and use of technology (UTAUT) -covers the technology supporting mechanisms initiated by an organisation to optimally support users in the use of the technology that supports the CoP (Venkatesh et al., 2003). However, facilitating conditions is more than only technological support. Stieglitz, Meske, \& Schallenmuller (20I3) pose that methodological support is also an important concept to take into consideration. This mainly concerns the support that community members receive when it comes to understanding the concept of a 'CoP'. Jeon, Kim, \& Koh (20II) show in their research on knowledge-sharing behaviour in CoPs that the facilitating conditions positively affect an individual's knowledgesharing behaviour. However, it is also plausible that optimal facilitating conditions also contribute to other types of social behaviour in CoPs, for example, in acquiring knowledge and discussing with colleagues. 


\subsubsection{Ability}

An individual's ability positively affects his or her exchange of know-how (Bigné et al., 20I5) and willingness to participate in collaborative projects (Bettiga et al., 2018). Cohen \& Levinthal (1990) claim that it is one's ability to recognize the value of new knowledge and information and the substantial processing and use of it that are fundamental in organisational learning and innovation. The ability to participate in knowledge management environments is often used simultaneously with self-efficacy (Wood \& Bandura, 2016), a concept coined by Bandura (1982) in the social cognitive theory. Wood \& Bandura (1989, p. 364) define perceived self-efficacy as "people's beliefs in their capabilities to mobilize the motivation, cognitive resources, and courses of action needed to exercise control over events in their lives".

Despite the strong similarities, there is also one fundamental difference between these two concepts. An individual's ability implies a proven value for his or her capability. Ability refers to a competence in doing, a skill, and a proficiency that has already been acquired; it represents the extent to which individuals have the required knowledge and skills to achieve a desired goal (Bettiga et al., 2018). However, self-efficacy refers to our confidence in the fact that we can perform a particular task, it represents our capability instead of our ability (Bandura, 1982). Evaluating the ability of an individual is often associated with testing the individual's ability to show a certain behaviour, which often requires experiments or tests. The perception of an individual about conducting behaviour can be evaluated with survey research and interviews. Ability in the MOA model is often operationalized as self-efficacy (e.g. Siemsen et al. (2008) and Turner \& Pennington (20I5)).

An important precondition for individual activity is understanding the information to be shared. Perceptions of useful information depend on the ability to effectively draw out relevant information and present it to a recipient, an ability to express. At the core of this is the sender's ability to manage organisational and personal information well (Marchand, Mey, Debruyne, Haene, \& Meul, 20I0). In the knowledge sharing context, the ability is described as the extent of one's skills and proficiencies required to share knowledge (Siemsen et al., 2008). According to Oshri, Fenema, \& Kotlarsky (2008), it is essential that participants have the expertise in encoding, storing, and retrieving information that enables knowledge transfer among distributed team members. However, an individual's ability to share knowledge differs in the extensiveness, complexity and diversity of the knowledge he or she possesses (Cohen \& Levinthal, 1990; Martin \& Salomon, 2003). For the acquirers of knowledge, this implies that having diversity of prior knowledge helps them absorb new knowledge, since it enhances the chance that the incoming knowledge is related to existing knowledge (Cohen \& Levinthal, 1990). Knowledge providers, with a high ability to utilize and share knowledge, are better equipped to understand how their knowledge may be of relevance in other contexts and therefore better able to frame the knowledge in a way that makes sense to potential acquirers (Reinholt et al., 20I I). In order to optimally participate in the CoP, other social characteristics are also desirable, in particular entering into social interaction with well-known and less well-known colleagues; the ability to contact (Rajagopal, Joosten-Ten Brinke, Van Bruggen \& Sloep, 20I2).

Providing relevant training that fits the needs and expectations of the community members contributes to the confidence in one's ability to perform and leads to increasing the activity of the individuals (Behringer \& Sassenberg, 20I5). Therefore, organisations often embed training sessions in the implementation strategy of the CoPs. However, the focus of these training sessions is often purely based on digital skills and less on psychological, sociological 
and communicational skills. An increasing group of organisations conducted both formal and informal training sessions to educate community members on improving their skills to actually be of value in the CoPs (Hildreth \& Kimble, 2004). A supportive organisation and a balanced strategy also contribute to the further optimization of individual's abilities (Flaman, Nykiforuk, Plotnikoff, \& Raine, 20I0).

The exploratory nature of this research project has led us to retain the concept of ability as part of the MOA model but to operationalize it by using self-efficacy variables. This is an approach that - consciously or unconsciously - has already been adopted by several researchers (Kettinger et al., 2015; Turner \& Pennington, 2015). The ability in this dissertation has been divided into three underlying constructs: ability to express, ability to contact and digital skills.

\subsection{Expanding our theoretical insights}

\subsection{Communality}

Initially, the MOA model was designed to provide insight into the influencing factors for social behaviour, which involves explaining behaviour but also sketching a framework or context. The opportunity factor plays an important role in this process. However, the opportunity factor primarily focuses on the influence of organisations, while CoPs also produce a unique atmosphere that is presumed to have a large influence on individual behaviour in groups. That is, the main reason that the factor communality is added to the MOA framework to ensure that the existence of a sense of community within or between organisations is present. In this thesis, 'sense of community' is interpreted as a concept similar to 'communality'.

People's sense of community stems from their perception of being linked into the dynamics of a complex system of relationships and interactions (Gilchrist, 2000). Pyrko, Dörfler, \& Eden (2017) introduce the concept of 'thinking together' as an important concept for increasing the possibility of developing learning partnerships and communality. The authors agree that communality cannot be set up by introducing a technological system that is compliant for hosting a CoP; the development of a CoP must be a constellation of learning processesexemplified in learning together practices-which contribute to a CoP's development.

This perception of being a member of a complex system (a community) is affected by many variables. In the remainder of this chapter, we briefly introduce a few of these variables and define and describe those that will form the basis in this thesis in more depth.A more elaborate description of the variables will be introduced in the chapter that focuses entirely on the community perspective (Chapter 8 ). The underlying theories to support the decision for these factors are the commitment and trust theory (Morgan \& Hunt, 1994), social influence model (Dholakia, Bagozzi, \& Pearo, 2004)s and sense of community theory (McMillan \& Chavis, I986).

In many academic articles on community development, trust is a construct that can be seen from various perspectives: trust in the community (Ardichvili, 2008; Lu, Zhao, \&Wang, 20 I0), trust in the organisation (Ardichvili, 2008) and trust in technology (McKnight, 2005). In this study, we will focus on trust in the community; Chen \& Hung (2010) define this interpersonal form of trust as a degree of belief in the good intentions, benevolence, competence, and reliability of members who share knowledge in professional virtual communities. Bista, Nepal, \& Paris (20I2) argue that trust is the foundation of every friendship. 
A lack of trust in social environments can work against the vision of connecting people to share their opinions and expressions (Caverlee, Liu, \& Webb, 2008). Without trust, members may not feel comfortable sharing their opinions. Bista, Nepal, \& Paris (20I2) introduce the concept of trust communities; this concept seeks to remove the lack of trust in others within the community and is built on the foundations of authenticity, open sharing, like-mindedness and mutual respect. As reported in the literature, trust is a critical factor in the success of communities. Without trust in place, members may be reluctant to share their knowledge or experiences with other community members due to the fear of misusing information and identities (Dwyer, Hiltz, \& Passerini, 2007).

In an online community, it takes time to achieve a level of comfort and trust, develop personal relationships, and evolve into a state of 'camaraderie' (Garrison \& Arbaugh, 2007). The advantage of a CoP compared to other types of communities is that a CoP is initiated by an organisation in which an extensive group already knows each other. A downside of this is that changing existing, sometimes trusted, standards becomes a complex task. Changing by fighting against an existing standard is often seen as a waste of time.A well-known quote from Buckminster Fuller confirms this:"You never change something by fighting the existing reality. To change something, build a new model that makes the existing model obsolete" (quoted in Andersson et al. (2009)). Clifton (1999) studied the level of trust in university-based learning communities and pointed out that the level of trust between members in the educational process should be high if a sense of communality is to develop: "When people do not trust each other, and when they do not share norms, obligations, and expectations, as is presently the case in many universities, the community is not likely to develop, and the self-interest of people in their status is likely to predominate". Kruse \& Louis (1993) agree with this point, as according to them, personal trust can build the sense of loyalty, commitment and effectiveness necessary for shared decisionmaking and the establishment of collegiality. Nias, Southworth \& Yeomans (1989) claimed that trust has two dimensions: predictability and common goals. These authors described these dimensions as follows: "For trust to exist, people must find one another highly predictable and share substantially the same aims". This is very similar to the way communities are organized around commonalities. Common standards, values, interests, and goals reinforce the confidence and trust members have in each other.

Thus, trust has often been reported as fundamental in the promotion of active social behaviour and the generation of meaningful communication within CoPs. It is often assumed that increasing trust is more likely to occur when community members share a common purpose, a sense of equality and collective ownership. One of the concepts that improves trust in the groups is shared leadership, comparable with a sense of equality. When leadership is shared among the colleagues, these same colleagues are more willing to openly talk about their daily progress and issues during daily meetings (Gholami, 20I5). It reflects a "web of mutual influencers and shared responsibility and is associated with enhanced outcomes in a variety of settings including work groups, virtual teams, and virtual collaborations" (Johnson et al., 20I5, p. I67). Another potential building block for the development of a sense of communality in a CoP is the perception of a shared identity. It is the shared domain-one of the components of a CoP, according to Wenger et al. (2002) - that contributes to the creation of a sense of shared identity (Kimmerle et al., 20I3). It is the collective identity that contributes to the development of best practices. The shared identity-or group identity-refers to the mutual cognitive state of community members, as well as the moral and emotional associations with the online community (Polletta \& Jasper, 200I). Following the reasoning of Sun, Rau, \& Ma (20I4), the communities with a stronger sense of shared identity usually have a greater number of member contributions. The level of 
trust is also reflected in this reasoning, since people with a shared identity are-according to Sun, Rau, \& Ma (2014) and Nahapiet \& Ghoshal (1998) -more likely to have similar goals, rules and interests and are therefore more likely to share information and participate in discussions.

A third concept related to the development of a sense of community is shared language. Community members need a shared language to obtain a mutual understanding of information during communication processes (Nahapiet \& Ghoshal, 1998). Liao \& Chou (201I) found significant evidence that those posting in a CoP are positively affected by social trust in other members and a shared language.A common vocabulary-consisting of explicit knowledge (e.g., of the meaning of words and terms) and tacit knowledge (e.g., of metaphors and symbols) - is an important conditional factor for the development of communality (Plaskoff, 2003).

Thus, the academic literature has shown that improving the level of trust is a matter of developing the sense of community. Communality - or the sense of community - can be studied from multiple perspectives. A critical reflection of these perspectives displays a considerable overlap. This leads to the use of three overarching variables that will be used in this study as supporting the development of a sense of community: shared leadership, shared identity, and a shared language.

\subsection{Social Behaviour in CoPs}

Activity is the crucial factor for the success of CoPs (Malinen, 2015; Wenger \& Snyder, 2000). In traditional offline CoPs, many individuals feel social pressure from other attendees to communicate, primarily because face-to-face communication is a rich medium with a physical audience. In online versions, it is much easier for an individual to observe what others share within the CoP than to make relevant contributions. Koh, Kim, Butler \& Bock (2000) identify a large group of passive participants and a smaller group of active participants, whereby the passive participants primarily 'view' content and the active participants 'post' content. Both groups are needed and reflect the members' level of commitment to the community (Koh \& Kim, 2004). As already pointed out, most individuals within online communities play the well-known role of lurker, with just a limited number of posters. However, due to the negative connotation and the often positive, implicit contributions of the lurker (Edelmann, Krimmer, \& Parycek, 2017), it has been decided to define them as observers instead of lurkers. The difference between posters and observers is that posters make contributions to the community by sending messages, videos or other media, while observers stay silent most of the time. Sun, Rau, \& Ma (20l4) follow the "90-9-I" principle, which states that in collaborative websites, such as an online community, $90 \%$ of the participants only read content, $9 \%$ of the participants edit content and $1 \%$ of the participants actively create new content. These numbers may differ in each community, but, in fact, despite the great potential of CoPs, many of the online environments have just a minimal number of active contributors. Nevertheless, this does not imply that all passive users have no value for the CoP.All community members who visit the online platform on a (less) frequent base can learn something from the material that is shared and apply it their practice (Sun et al., 20l4).

This statement is in accordance with the operationalization of social behaviour introduced by Lampe, Wash, Velasquez, \& Ozkaya (2010). These authors define one base measure of social behaviour as the creation of an account in a system.After creating an account in the system, one can decide to participate actively ("social interactors") or to mainly consume content by reading posts, watching videos, or reading articles-all without contributing to the production of the 
resources they use ("knowledge acquirers"). Hooff \& Ridder (2004) also made the distinction between knowledge collection and knowledge donation. Whereas knowledge donation is defined as transforming one's personal intellectual capital into explicit knowledge, knowledge collection (implicit or explicit) involves soliciting colleagues to share their intellectual capital. Knowledge creation and exchange are identified as hallmarks of CoPs (Pan et al., 2015).A third form of activity-which is seen increasingly in CoPs-is networking activity (Bock, Robert, \& Kim, 2005; Pan et al., 20 I5). Networking activity is centred around the idea of establishing contact with other CoP members (Zboralski et al., 2006).

Ang (20I I) combined each type of social behaviour in the community in his 4C model, which represents the following activities: connectivity, conversation, collaboration and content creation. Since some individuals mainly observe interactions in a community, for this research project, we created a transcendent approach with consumption, connectivity, conversation, collaboration and content creation as the social activities in a communal environment. This will be quantified in surveys by asking professionals about their social behaviour in CoPs and by using a subset of the most commonly employed quantitative measures, defined as duration of membership, time spent online, number of visits, number of hits/views of content, number of contributions, and density of social interaction with others (Malinen, 20I5). In this dissertation, the social interactors and knowledge acquirers are used as the starting point for the distinction in social activity: social interaction behaviour and knowledge acquisition behaviour.

\subsection{Conclusion and Conceptual model}

In this chapter, the main objective was to get an answer on the first sub-question in this research project:

Which factors potentially affect the social behaviour of community members in communities of practice?

In this dissertation, we have chosen to create an explorative, conceptual, integrated model based on the motivation, opportunity \& ability theory (Boxall \& Purcell, 20I I; Siemsen et al., 2008), the social influence model (Fulk, Schmitz \& Steinfield, 1990), the social exchange theory (Emerson, 1976), and the social capital theory (Nahapiet \& Ghosal, 1998). and that will be tested in various studies and cases. This model that is further referred to as the Motivation, Opportunity, Ability, Communality model (MOAC model) is shown in Figure 3.I. and forms the basis for the studies described in the coming chapters.

To facilitate a successful CoP, the presence of motivated and capable individuals is essential. Siemsen et al. (2008) posit that motivation, opportunity and ability (MOA-framework) are crucial factors for knowledge-sharing behaviour among employees. This research has been conducted within the operational context of inter-employee knowledge sharing. A definite shortcoming of this study is the measurement of the three factors: motivation, opportunity and ability. For example, opportunity is measured only by time availability, thus it remains only a proxy for opportunity. Siemsen et al. (2008) introduce other opportunity-related considerations such as space and or resource, as potentially relevant factors for knowledge sharing in an organisational setting. However, another limitation of the MOA framework is that it is a generic approach for determining and explaining certain activities. To refine the model, we suggest specific contextual factors related to this study, particularly communality but also specifications for the other constructs such as work autonomy and facilitating conditions for the construct opportunity. The independent variable communality describes the common denominator of the participants 
within a CoP; the common denominator is divided into shared leadership, shared identity, and shared language.

In pursuing their interests within their domain, practitioners in a CoP engage in joint activities and discussions, help each other, and share information (Wenger, Trayner \& De Laat, 20II). However, the focus of Wenger, Trayner \& De Laat, $(20 \mathrm{II})$ is on sharing knowledge, while social behaviour is more than only sharing knowledge.Acquiring knowledge, establishing relationships and consulting others when questions arise are also types of social behaviour that occur within a CoP.Therefore, in this dissertation, the distinction has been made between social interaction behaviour and knowledge acquisition behaviour, which represents the two typical forms of participation in CoPs (Chapters 6 to 8 ).

In addition to that, the MOAC variables are also applied to group behaviour in CoPs, which are represented in community network positions and social interaction patterns. The specific background of these applications of the MOAC model are available in the Chapters 10 and II .

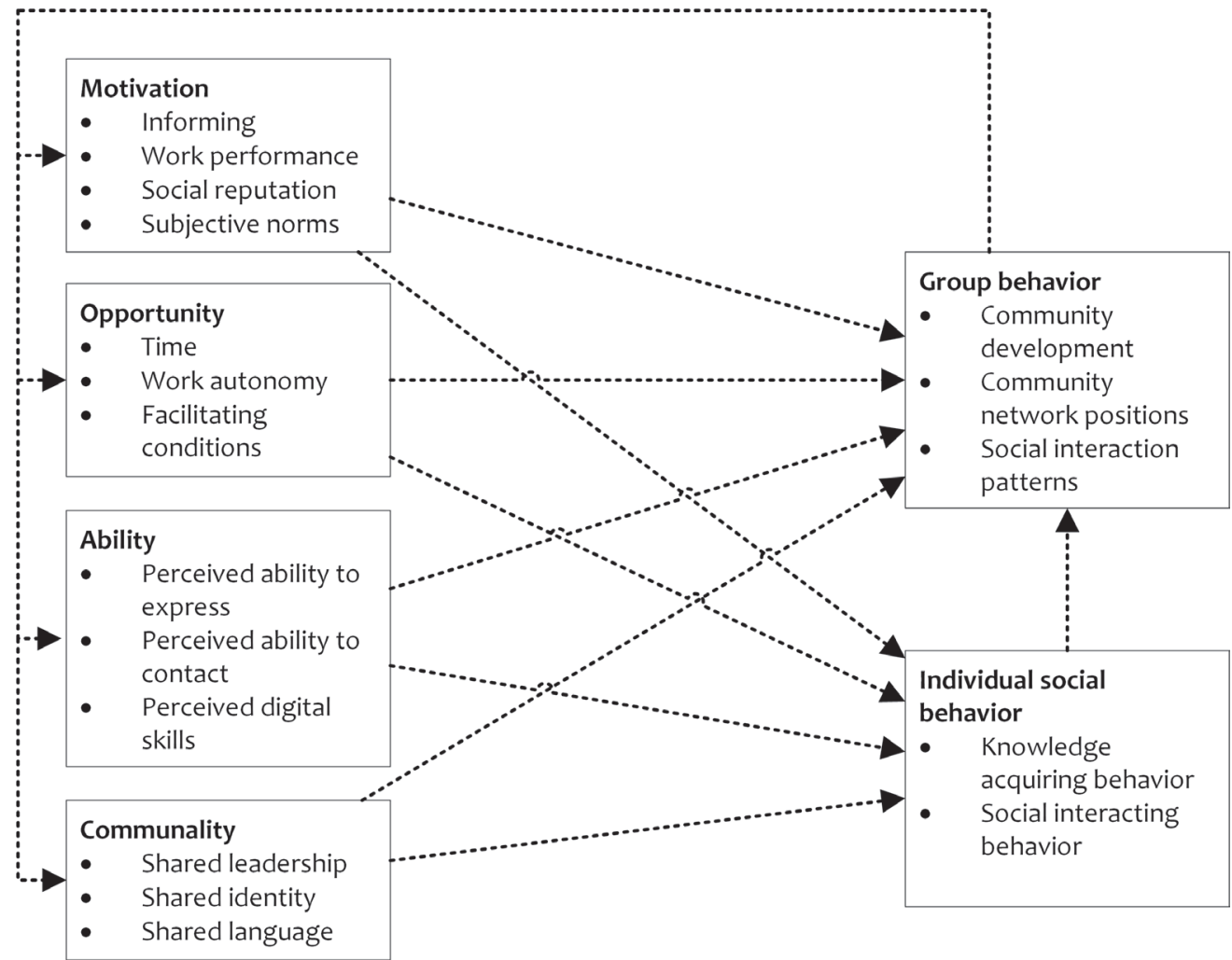

Figure 3.I. Community of Practice Development Model 



\section{PART II: RESEARCH METHODOLOGY}

Part II aims to describe the research methodology in this dissertation. This exploratory research is being carried out in a number of organisations that to a greater or lesser extent support the development of communities of practice in their organisations. In Chapter 4, the focus is on describing the context of the four central communities of practice.

Chapter 5 describes the community monitoring and evaluation methodology which aims to test, monitor and evaluate the (individual and group) processes in communities of practice.
Parts of this dissertation will be used for future scientific publications 



\section{4 \\ Selection of Cases}

\section{I. Introduction}

In this chapter, we will provide an outline of the selection process and an extensive description of the background of the four main cases in this dissertation. In this case-based dissertation, four cases are central in the chapters 6, 7, 8, 10 and II. In Chapter 9 - a chapter predominantly focused on examining general and specific community development patterns in CoPs - the four cases are evaluated, and three other cases were added. The focus in the description of the four cases is on goals and objectives, implementation strategies, and organisational culture. These criteria contribute to our understanding of the goals, strategies and backgrounds of the organisations that have introduced CoPs.

\subsection{Evaluation Frameworks}

\subsection{Community Infrastructure Framework}

The selection process of the cases in this dissertation was performed based on the online community technology used by the CoPs. The community infrastructure framework describes the criteria that online community technology must meet to be truly supportive in the development of CoPs. Analysing technological community infrastructures can be done by subdividing them into smaller units, as outlined in Chapter 2 (section 2.5. Community Infrastructure). The cases in this dissertation all use the same online community software from Winkwaves Harmonics, a provider of online community technology in the Netherlands. This organisation provides a platform and consultancy for implementing CoPs in organisations. Knowledge-driven organisations can use the platform for the development of CoPs. Nevertheless, this platform contrasts with existing intranets specifically developed for individual collaboration. Users can create communities of interest and practice to improve practices and solve problems. In this research, a selected sample of communities were selected as the subject of the research in order to define factors that affect the development of these communities. These cases were carefully selected after a selection interview with the community managers to determine whether the cases are currently developing or have already developed towards a CoP.

This social technology meets the main criteria set as a precondition for letting social technology become a platform for CoPs. In Table 4.I, we check the extent to which the specified social technology meets these standards. 
Table 4. I

Accordance with technology criteria

\section{Technology criteria}

\section{Reference}

Wenger, White, Smith, \&

Rowe, (2005), Bakardjieva \&

Feenberg (2002) \& Gongla \&

Rizzuto (200I) communal repositories (e.g.,
Wenger, White, Smith, \& Rowe, (2005), Bakardjieva \& Feenberg (2002) \& Gongla \& Rizzuto (200I)

\section{Meets this criterion} (Y/N)
Publishing, sharing and searching: options for knowledge management in document management)

Social interaction: options to discuss with others via discussion boards

Tending: create profiles, options to interact on private and public spheres and in open and closed groups
Yes, plenty of options for social interaction between profiles in the CoPs

\section{Yes, document management} system available and searchable in groups.
Wenger, White, Smith, \& Rowe, (2005), Bakardjieva \& Feenberg (2002) \& Gongla \& Rizzuto (200I)
Yes, community members can interact with each other in both private (chats) and public conversations (e.g. posts \& comments)

\subsubsection{Community Foundation Framework}

A flexible, results-driven and committed organisation is often seen as the basis for a wellfunctioning CoP.The organisation must provide a solid basis that supports collective activities, stimulate initiative and provide opportunities. The organisations participating in this research program differ from each other in context but also in the phase in which each community is currently at. Since the context of these cases is rather different, we interviewed the initiators within each community to describe the background factors that might be a descriptor of the results. A set of factors has been composed to clarify the image of the context in which certain developments in the CoPs occur. These factors are: organisational goals and objectives, implementation strategy, and organisational culture. Based on these factors, we describe the four CoPs that play a major role in this dissertation. By means of semi-structured interviews with at least the community manager of the organisation a profound image of the context beyond the CoP has emerged. In addition, the annual reports, whether available or not, were consulted to provide a good indication of the (dis)similarities between the cases, and the webbased social interaction data provided additional numerical data. The data generated in the interviews and from the annual reports are summarized in Table 4.I.

\subsection{Case description}

In the following paragraphs, the four primary cases in this study will be described. These four cases are evaluated based on the following factors: organisational goals and objectives, implementation strategy, and organisational culture. 


\subsection{CoP in a financial service organisation}

Technological developments, changes in the market, economic and political developments and changing customer needs contribute to the need for financial organisations to develop and adapt to the changing world. All these developments contribute to the need for adaptability to changing circumstances, an optimal exchange of knowledge and close cooperation within and between departments. Since the financial service organisation operates worldwide, an annual report specifically for the Netherlands is missing. As a result, the exact number of employees at the organisation is unknown. However, in the interview with the community managers, it was stated that every employee was automatically registered for the CoP, and the total number of employees in the CoP was 270.

\section{Organisational culture}

The financial service organisation that participated in this study offers online customer support and is therefore centralized at one location based in the Netherlands. The culture in the organisation can be categorised as a market-driven culture, which implies that it concentrates on stability and control with an external focus. The financial market is a highly competitive market in which there is a continuous battle to tempt (loyal) customers to commit themselves to the organisation.

\section{Organisational goals and objectives for the CoP}

Before implementing the CoP, the organisation has gone through some reorganisations that resulted in declining commitment of the remaining professionals. Three objectives were set by the organisation when deciding to implement an online community software supporting the development of CoPs. First, to prevent the organisation from experiencing a 'brain drain', second, to strengthen the mutual understanding to overcome the commitment issues existing in the organisation, and third, in the longer term to stimulate intensive collaboration between teams and departments. These objectives can be summarized in two objectives defined in Chapter 2: improve collective learning and create more efficient knowledge management.

\section{Implementation strategy}

An online community software has been purchased to ensure that professionals are given the opportunity to exchange knowledge, discuss relevant topics, collaborate on the development of (new) products and services and interact socially in an unforced way. The replaced intranet no longer meets the requirements that are currently being asked, and it was therefore replaced by a more social package. The changing situation in the organisation required a thorough implementation strategy. An implementation strategy was designed in collaboration with the provider of the online community software to increase the chances of success. The resulting development-based approach resulted in a couple of iterative steps. One of the first steps was to create a division of roles regarding fulfilling community manager roles. In total, twelve professionals were assigned as community managers and received training from the provider of the online community software and a community leader regarding the underlying social model, technical foundations and use of the platform. The community leader was responsible for the transfer of knowledge from one platform to the other, and the other community managers were responsible for motivating peers to join the CoP in an active manner. All employees in the organisation were automatically enrolled in the CoP; thus, the focus of the community managers is encouraging their colleagues to participate. 
Activities organized to stimulate social interaction varied from offline workshops to online $\mathrm{Q} \& A$ sessions.According to the interviewees, the result has been an increasingly active group of professionals sharing procedures, updates, and subject-specific knowledge and the development of social and professional network.

\subsubsection{CoP in a large hospital}

In general, all hospitals aim to be patient-oriented and safe. In this respect, the hospital in question is no different.The hospital in this case focuses on providing not only high-quality patient care but also on excellent training and research to ensure that the healthcare in the region continuously meets the highest quality requirements. Continuous professional development programmes are initiated that aim to contribute to the ambitions. Social technology has been purchased to bridge the boundaries between departments and to join forces across the borders of expertise and experiences. The annual report shows that 3.577 employees work at the organisation, of which 818 employees are registered in the CoP.

\section{Organisational culture}

Since it is crucial that cooperation within and between departments is optimal and the responsibilities are spoken for, a hierarchical organisational culture is often the result. This culture can be characterized as one with an internal mind-set and with a focus on stability. To realize the ambition to improve cooperation between departments, various approaches can be chosen. The organisational structure of a Dutch hospital is largely determined by the method of financing and by the medical specialists at the hospital. The medical specialists may be employed by a hospital, but more often, they are independent professionals. Groups of these independent specialists in a hospital are united in partnerships by specialty. Since medical specialists are not formally employed by the hospital, the hospital has less control over the individual social behaviour of these specialists especially regarding other employment supporting activities such as CoPs.

\section{Organisational goals and objectives for the CoP}

The organisation in this study has chosen an approach that requires close cooperation across the borders of different departments within the hospital, which closely resembles the objective of improving collective learning. Another objective set to be fulfilled by the CoP is the goal to increase the opportunities for professional networking. The size of the organisation means that realizing cooperation between the experts is a complex task. Even though certain knowledge areas have a strong overlap, cooperation is desirable. Especially in complex issues where different knowledge domains come together, it is crucial to have insight into the expertise of colleagues from other departments, as this might contribute to resolving the issue. The possibility of expanding professional networks contributes to this activity.

\section{Implementation strategy}

The first steps towards fulfilling the ambition to improve the open, professional learning atmosphere of the organisation were set by the organisation management board. The organisation's management decided to implement a social intranet aimed at supporting the communication, collaboration and knowledge management of professionals and, ultimately, breaking barriers between departments within the organisation. Reinventing the wheel is one of the pitfalls in an organisation when the focus is on finding a solution for a certain recurring problem. Therefore, the organisation in question embraced a solution with a crossing-border initiative where representatives from different departments join forces in finding solutions for 
recurring problems. However, the organisation does not solely rely on the advantages of a social intranet for knowledge management. Due to the many changes in management positions and incongruent communication between departments and stakeholders, the organisation has decided to purchase two slightly overlapping systems. One system is for knowledge management, and one system is for knowledge exchange, discussion, collaboration and networking. These two systems, which have overlapping characteristics, cause incomprehension and ambiguity among the final users, making the users choose only one of the two systems.

The implementation of online community software for the CoP has come under the responsibility of one of the communication professionals. The central message during the implementation of the CoP was the question of what such an initiative contributes to the practice of the professionals in an organisation. Roughly speaking, two approaches were initiated to create awareness of the added value: focus on mass-communication sessions and demand-driven sessions. The complexity of the organisation-with more than 3000 employees in different forms of employment and contract types-has as the consequence that an implementation strategy with massive information sessions and inspiration sessions are needed to create awareness of the existence of the CoP on a more extensive scale. Based on these mass sessions, individuals are inspired to create sub-communities within their own departments. Inspired individuals in the organisation who demand more progressive insights into the possibilities of the CoP organize more advanced sessions in cooperation with the community manager. It is the ambition of the community manager to spread the enthusiasm for participating in the CoP beyond these more personal counselling sessions to the larger organisation. To stimulate the rapid growth of the CoP, the community manager has recruited those enthusiastic individuals as ambassadors within the organisation. The implementation strategy can be characterized as a typical diffusion approach, where it is expected that the enthusiasm of others contributes to the implementation of the community thinking within and between other departments in the hospital. The distinctive departments within a hospital (e.g., surgery, nursing, communication, administration) result in a multiplicity of practices. However, in general, the CoP is used for procedures, scheduling, internal communication, subject-specific knowledge and networking.

\subsubsection{CoP in a youth care institution}

The youth care institution in this project is an organisation that supports children and their parents - regardless of their situation or background - to create a safe environment for the children in which they are loved, counted and given opportunities to develop themselves. In complex situations, the professionals in the youth care institution focus primarily on the wellbeing of the family and not on the type of care assistance. This approach leads to extremely context-dependent situations and cases in which the professionals could optimally benefit from the knowledge and experiences gained within the teams.A total of 995 employees are indicated in the annual report, of which 538 are registered in the CoP.

\section{Organisational culture}

The professionals work in teams without hierarchy and countless rules. Each person is responsible for his or her own development and the added value he or she can contribute to the organisation and in the different cases. The organisational culture can best be characterized as a clan culture, which implies a high internal focus and high flexibility. The important goal in the organisation is creating a good, open and accessible culture in which barriers between professionals are dismantled when necessary. The focus is on collaboration, engagement and unity. The varied, complex and sometimes moving cases require excellent cooperation between 
the professionals and an atmosphere where important situations can be discussed.

The youth care institution that participated in this study is a decentralized organisation that has decided to reorganize into self-organizing teams. Employees in the organisation have, in general, one of two types of employment contracts: permanent and based on secondment. An internal study in the organisation has shown that employees with a permanent contract have a higher sense of commitment to the organisation than colleagues who work on a secondment basis. Most professionals in the organisation work in autonomous teams mostly present in the field. However, a significant number of support staff also work at the head office of this networked organisation. The substantive diversity of positions within the organisation ensures that the opportunity for mutual knowledge transfer has great potential. The mutual dependency in the work processes contributes to this.

\section{Organisational goals and objectives for the CoP}

Creating a good environment in which employees can work according to the 'activity-based working' (Dutch: Nieuwe Werken) paradigm is one of the main drivers for starting a CoP. Therefore, the organisation decided to introduce online community software that is available in a time- and place-independent manner. By using online community software, the organisation tries to close the gap between organisational ambitions and the use of modern technology. The role of the CoP is continuously considered when solving these organisational issues. Concrete and specific organisational objectives have not been formulated, but the organisation is increasingly transforming its professional approach regarding the CoP. This implies that a future step will be to formulate and quantify the success criteria of the CoP for individuals and organisations. However, reading between the lines, it becomes clear that the CoP is mainly initiated to improve collective learning, make knowledge management more efficient and increase opportunities for professional networking.

\section{Implementation strategy}

The shift towards a new self-organizing form of working has also had consequences for the implementation strategy. In the transition from management-driven decisions to self-managing and self-organizing teams, choices have been made regarding information provision and collaboration-supporting technologies. Professionals in the organisation are not forced to participate in the community, but the information provision runs only through the community. This directive implementation strategy was combined with a development implementation strategy-a strategy based on increasing the competences of the organisational members to make them capable of using social technology in a meaningful manner.

Within the community, the focus is mainly on disseminating, developing, improving and repeating successful activities. The less successful initiatives initiated in the CoP are often not continued. For the time being, the social behaviour of the community members is limited to sharing the contributions of others, responding to 'less substantive' material and connecting demand to supply. Substantial contributions in the form of posts and comments remain somewhat behind. A plausible explanation given during the interview is the phenomenon of "cold feed", by which the interviewee meant participants' fear of stepping outside their comfort zone. In addition, the community coach also sees that a considerable number of community members lack the selfconfidence to respond critically to their colleagues or to give a professional opinion in general. The community management board, which consists of representatives from the communication department and the HR department, takes the initiative to organize training sessions to support community members in their first steps towards active community membership. The 
goal of these training sessions is to reduce the barriers to expressing themselves in an online environment.

The community coach sometimes gives many members a little push from behind to overcome their initial reluctance. However, the community coach wants to give an example of the way in which CoPs can be fully utilized to achieve individual and organisational objectives. Activities used to realize this are creating awareness of the possibilities of CoPs, stimulating activity, supporting initiatives and training new members. In the organisation, a conscious choice has been made to use the title community coach instead of manager. The community coach is not the one who determines what the social intranet looks like or that colleagues should use it. The community coach only supports their colleagues in answering the question of how they can use the CoP to make a more meaningful workplace for themselves and for their department. All these initiatives are carried out based on employee requests, which guides the choice to participate in a meaningful manner in the CoP.

The purpose of these efforts is to increase awareness in the organisation and to achieve more social interaction between the members. Some specific examples are working on treatment programmes and procedures, updating recurring practices, forming social connections between community members, and exchanging subject-specific knowledge. The aim of the community management is to further limit the role of the community manager. This should ultimately lead to the organic growth of the CoP towards a meaningful place for all members.

\subsubsection{CoP in an organisation for the care of disabled people}

The fourth case is an organisation that helps people with disabilities to have an active role in society. It helps such people gain and maintain control over their lives by, for example, strengthening their own perseverance and increasing self-reliance. In addition, the organisation uses the knowledge and experiences from healthcare professionals to better structure society for people with disabilities. The expertise in the organisation varies from care of people with intellectual or physical disabilities to those with chronic illnesses, non-congenital brain injuries or forms of autism. The annual report shows that the organisation consists of 752 employees. Of these, 273 people are registered in the CoP.

\section{Organisational culture}

The organisational culture in which the CoP has been introduced can be characterized as hierarchical. This is evident in their internal focus and their concentration on creating a stable organisation. The teams of caregivers and consultants use the online environment as their primary source for information relevant for performing their jobs as efficiently as possible. Since all the professionals receive the needed devices (mobile phones, tablets and/or computers) to access the online environment, members are always able to visit the online environment.

Organisational goals and objectives for the CoP

The different knowledge areas in the organisation ensure that colleagues can not only learn from peers within their own field of expertise (improve the collective learning) but also acquire knowledge about, for example, procedures, protocols, and methods outside their own knowledge domains and departments (more efficient knowledge management). Therefore, the management board in the organisation decided to initiate an environment that supports the professionals in the organisation in collaborating across the borders of their own expertise. 


\section{Implementation strategy}

The CoP was implemented in addition to an existing document management system and introduced as a replacement for a classical intranet. The interactive implementation strategy was established in collaboration with the provider of the online community software and with the general community management board, which consists of four persons. These four persons are responsible for community development. The role of the community managers and the ambassadors is primarily supportive in nature instead of prescriptive and moderating. Within the organisation, the self-correcting ability of the employees is relied upon. If there is indecent behaviour, it is assumed that colleagues correct each other.

In the elaboration of the implementation strategy, a select group of managers in the organisation were initially designated as ambassadors of the community within the departments. They were assigned to take on an exemplary role for colleagues, using all kinds of methods and techniques to engage their colleagues. Individuals with a naturally enthusiastic attitude towards knowledge sharing, cooperation and development played a fundamental role in their departments. A result of this is that the less-enthusiastic managers in particular have a less-active group of colleagues in the online environment.

Professionals in the organisation are not forced to participate but have the freedom to explore the possibilities and decide for themselves how they will act in regard to this new form of working. However, most professionals currently participate in the online environment. The fact that many joint activities take place online could be an indicator of this development. The practices in the organisation that are relevant for the care of disabled people mainly focus on mutual introduction, procedures, recent updates, internal communication, contentspecific topics, and networking. At the time of the study, these initiatives were mainly initiated by the active group of participants who see the possibilities of the CoP. The objective of the community management is to further integrate the possibilities of the CoP into the workflows in the organisation.

\subsection{Summary}

The organisations participating in the studies in this dissertation have similarities and differences. These similarities and differences will ultimately influence developments within the CoPs. In particular, the difference between the number of employees and the registered community members gives an indication of the importance of the CoP to the employees. The goals and objectives formulated by the organisations show that the interests of each organisation are different-a phenomenon that may arise from the phase in which the CoP finds itself and the degree of maturity of the CoP.The different implementation strategies within the organisation show that in practice there is no uniform picture of the 'ideal strategy', but the differences and similarities will have an impact on the developments. The last concept used to evaluate CoPs is organisational culture. It is assumed that the differences in the cultures have a considerable impact on the acceptance, adoption and use of the CoPs in the daily work of the community members. 
Table 4.2

Accordance with community constructs

\begin{tabular}{|c|c|c|c|c|}
\hline $\begin{array}{l}\text { Community } \\
\text { development } \\
\text { constructs }\end{array}$ & $\begin{array}{l}\text { Financial } \\
\text { service } \\
\text { organisation }\end{array}$ & $\begin{array}{l}\text { Large } \\
\text { hospital }\end{array}$ & $\begin{array}{l}\text { Youth care } \\
\text { institution }\end{array}$ & $\begin{array}{l}\text { Organisation } \\
\text { for the care } \\
\text { of disabled } \\
\text { people }\end{array}$ \\
\hline $\begin{array}{l}\text { Start date of } \\
\text { the CoP }\end{array}$ & 2014 & 2013 & 2013 & 2015 \\
\hline $\begin{array}{l}\text { Number of } \\
\text { employees }\end{array}$ & 270 & 3577 & 995 & 752 \\
\hline $\begin{array}{l}\text { Number of } \\
\text { registered } \\
\text { community } \\
\text { members }\end{array}$ & 270 & 818 & 538 & 273 \\
\hline $\begin{array}{l}\text { Goals and } \\
\text { objectives }\end{array}$ & $\begin{array}{l}\text { - Improve } \\
\text { collective } \\
\text { learning } \\
\text { - More efficient } \\
\text { knowledge } \\
\text { management }\end{array}$ & $\begin{array}{l}\text { - Improve } \\
\text { collective } \\
\text { learning } \\
\text { - Increased } \\
\text { opportunities } \\
\text { for professional } \\
\text { networking }\end{array}$ & $\begin{array}{l}\text { - Improve } \\
\text { collective } \\
\text { learning } \\
\text { - More efficient } \\
\text { knowledge } \\
\text { management } \\
\text { - Increased } \\
\text { opportunities } \\
\text { for professional } \\
\text { networking }\end{array}$ & $\begin{array}{l}\text { - Improve } \\
\text { collective } \\
\text { learning } \\
\text { - More efficient } \\
\text { knowledge } \\
\text { management }\end{array}$ \\
\hline
\end{tabular}

\begin{tabular}{lllll}
\hline $\begin{array}{l}\text { Implementation } \\
\text { strategy }\end{array}$ & $\begin{array}{l}\text { Development } \\
\text { approach }\end{array}$ & $\begin{array}{l}\text { Diffusion } \\
\text { approach }\end{array}$ & $\begin{array}{l}\text { Combination of } \\
\text { a directive and } \\
\text { development } \\
\text { approach }\end{array}$ & $\begin{array}{l}\text { Interactive } \\
\text { approach }\end{array}$ \\
\hline $\begin{array}{l}\text { Organisational } \\
\text { culture }\end{array}$ & Market & Hierarchy & Clan & Hierarchy \\
\hline
\end{tabular}





\section{5 \\ Community Monitoring and Evaluation Methodology}

\section{I. Introduction}

This exploratory research employs the design, development and evaluation system of the community monitoring and evaluation methodology (CMEM). Unique to this methodology are the multi-method approach by which the developments in CoPs are approached; the combination of subjective data with objective data, whereby the subjectivity is expressed by means of the MOAC model in the individual motivations, the possibilities offered, the self-efficacy, and the individual sense of communality combined with the objective data representing the actual individual behaviour in CoPs; and the extent to which the methodology is data-driven. To create an intersubjective approach that is replicable for other researchers, the chosen approach is described step by step so that the degree of the subjective interpretation of the researcher is reduced. In this chapter, a basis will be given for the methods and techniques used to evaluate and monitor developments in CoPs in an increasingly data-driven manner. These efforts should contribute to answering the following research question: What is a relevant methodology for the monitoring and evaluation of individual and group behaviour in communities of practice? Each chapter examines another part of community development in which an appropriate method is applied and/or developed. In the final conclusions and discussion (Chapter 12), an answer will be provided to the research question.

This chapter describes the relationship between the research methods, which is intended to lead to a community monitoring and evaluation methodology. In section 5.2, an outline of the research approach is given, whereby the approach has been divided into two parts: (I) the individual social behaviour in CoPs and (2) the group behaviour in CoPs. Insight into both perspectives on social behaviour increases our knowledge of developments within the CoPs. Within this research approach, this research consists of qualitative (interviews), quantitative (survey), and relational (web-based social interaction) data collection and analysis. In a hybrid approach that entails all three data sources, a predominantly data-driven approach has been designed that acts as a community monitoring and evaluation methodology. In sum, the main purpose of the developed research methodology is to structure and guide the exploratory process regarding the way to analyse, monitor and evaluate the social behaviour - development - in CoPs as reported in this dissertation.

\subsection{CMEM Framework}

The research approach used for the purpose of the CMEM aims to monitor and evaluate the developments in CoPs as accurately as possible. We approach CoP development as a continuous process in which collaborations are organized, in-depth content or organisational knowledge is developed and social (professional) networks are built and maintained.The data and analytics used in the CMEM can be viewed from four perspectives (Figure 5.I) using just as many types of analytics: descriptive analytics, diagnostic analytics, predictive analytics, and prescriptive analytics (Banerjee, Bandyopadhyay, \& Acharya, 2013). These authors used these four categories as the 
basis for business analytics, which is a term that is defined as "a set of all the skills, technologies, applications and practices required for continuous iterative exploration and investigation of past business performance to gain insight and drive business planning" (Banerjee et al., 20I3, p.l.). In the field of business analytics, much research has been conducted on the possibility of making meaningful use of existing, largely objective data. The approaches that were developed through the CMEM were applied Figure 5.I. Types of community analytics to the evaluation and (adapted from Banerjee, et al, 2013) monitoring of CoPs.

This dissertation is a first step towards a real-time, data-driven and intersubjective monitoring and evaluation methodology for the analysis of developments within CoPs. The methods included in the methodology can be separated along the continuum of the community analytics. First, by evaluating relevant factors affecting an individual's social behaviour in CoPs, diagnostic and prescriptive community analytics will be collected. Second, by monitoring and evaluating the developments in CoPs and the impact of individual members, descriptive and prescriptive community analytics will be collected. Third, the evaluation of individual social network positions in CoPs, lead both to descriptive and diagnostic community analytics, and the last study to evaluate social interaction patterns in CoPs result in descriptive, diagnostic and prescriptive community analytics. These four areas focus on two levels of social behaviour: individual social behaviour and group behaviour.

This chapter discusses the first step towards a community monitoring and evaluation methodology that should ultimately be applicable in a practical manner to both practice and science. In the following paragraphs, the focus will be on further detailing the various components that will be included in the first version of the community monitoring and evaluation model.

\subsection{Individual Social Behaviour in Communities of Practice}

\subsection{Evaluate relevant factors affecting an individual's social behaviour in CoPs}

The first part of this chapter describes the first community development perspective in the CMEM: evaluating the relevant factors affecting an individual's social behaviour in CoPs. The method of research used for this purpose is a survey among community members of four CoPs in the Netherlands. The conceptual MOAC framework introduced in Chapter 3 forms the basis for the cross-sectional survey, which aims to identify those factors that contribute to the decision of the individual to participate in a CoP.These factors that are covered in the MOAC framework are divided into three levels: the individual level, the organisational level, and the collective level.

In general, surveys are useful for describing the characteristics of a large population but also for providing insight into individual characteristics, thoughts, and perceptions (Dainton \& Zelley, 2005). However, surveys are also readily used for self-reporting on, for example, one's own 
behaviour and performance (Hildreth \& Kimble, 2004). Thus, "one of the real advantages of quantitative methods (e.g., survey research) is their ability to use smaller groups of people to make inferences about larger groups that would be prohibitively expensive to study" (Holton \& Burnett, 1997, p. 7I). Insight into individual perceptions provides a better picture of the background of individual social behaviour. To better understand the reasoning behind certain community developments, it is therefore useful to first evaluate individual perceptions. This can be done by means of, for example, polls, interviews, and questionnaires, the last of which was chosen for the purpose of the CMEM.

In four distinctive cases - all of which use the same online community software to support the CoPs - a standardized survey was distributed to test the model among the employees. These four cases differ in their domains: a hospital, a financial banking institution, a youth care institution and an institution for the care of disabled people. In addition to the domain, the size, structure, culture and implementation strategies differ as well. Interviews were conducted with the community managers involved in the four cases to determine the role and position of the CoP within the policies of the organisation. These interviews and their implications are described in Chapter 4 and have an important function in outlining why certain individual and group behaviours occur.

For the questionnaire, the underlying variables from the MOAC model belonging to motivation and ability (individual perspective), opportunity (organisation perspective), and communality (collective perspective) are tested with a sample of the four organisations $(n=22 \mathrm{l})$. For research on individuals' social behaviours, it has been decided to approach the MOAC model using the four underlying perspectives instead of using the model as an overarching explanatory model for individual social behaviour. The scale developed in this thesis not only provides insight into the existing components defined in the MOA model (motivation, opportunity, ability and the communality defined in this study), but also into more specific underlying variables in order to provide more insight into the background of individual behaviour. Opportunity is - for example - further subdivided into time offered, work autonomy, and facilitating conditions. The results from this survey enable us to form a meaningful picture of the motives for using CoPs and factors that influence individual social behaviour.

In Chapters 6,7 and 8, the individual, organisational and community factors that affect the social behaviour of community members in a CoP will be examined. The fact that the social activity of the community members in CoPs is generally not normally distributed and that the MOAC framework has been greatly expanded has led us to decide to use a partial least squares structural equation modelling approach (PLS-SEM). PLS-SEM is an appropriate statistical test when identifying the constructs driving, for example, a certain type of social behaviour and when the research is exploratory in nature (Hair, Ringle, \& Sarstedt, 20 I I). Another advantage of the PLS-SEM compared to the co-varianced based SEM techniques is the required sample size. In case of PLS-SEM a sample of ten times the number of structural paths in the structural model (Hair et al., 20I I). In case of small samples size, PLS-SEM generally provides "higher levels of statistical power and reaches convergence much more often than CB-SEM (Sarstedt, Ringle, Smith, Reams, \& Hair, 20I4, p. 107). In this dissertation, the test is used to estimate a complex cause-effect relationship model with changing latent variables for each chapter. An elaboration of the latent variables is discussed in the accompanying chapters $6,7,8,10$ and II. 


\subsection{Group Behaviour in Communities of Practice}

The digital traces that community members leave within CoPs stored in web-based social interaction data show behavioural patterns that provide insight into developments within CoPs at the group level. Creating an account, visiting a page or posting a comment are typical examples of these registered social interactions. In this part of the dissertation, these data are transformed into meaningful data that provide insight into developments within CoPs from different angles. This section describes the background and coherence between the methods used.

Our society is creating tremendous amounts of data every day. These data often provide an intriguing insight into the social behaviour of humans. New approaches are developed in different research disciplines to use these data meaningfully. These approaches to processing these data formats and structures often differ from those typically used in a given field, thus creating methodological advances (Mahrt \& Scharkow, 20I3). In recent decades, many academics have started initiatives to make meaningful use of these new data sources. For example, in the form of social network analysis, social media analytics, trend discovery, storytelling, discourse analysis and other forms of (social) text mining (Cambria, Rajagopal, Olsher, \& Das, 20 I4; Hafeez \& Alghatas, 2007) have been developed. Bode, Sutton, Lacey, Fennell, \& Leonards (2017), for example, used the data from social networking sites to identify patterns in social behaviour, while Vollenbroek \& De Vries (2016) combined text mining with social network data to create and analyse two-mode network graphs. Identifying and interpreting patterns provides reliable insight into developments in social environments. However, this rather objective reflection of reality provides a first indication of how a given situation is structured.The subjective perception of individuals about their own social behaviours offers extra insight that can be used to explain the behaviour.

Many existing studies focusing on social behaviour try to explain a given social behaviour by observing sequential or synchronous actions of individuals; typical examples are ethnographic (Ceaser, 2018) and netnographic research (Langer \& Beckman, 2005). Other studies mainly focus on intentional social behaviour or attitudes towards certain social behaviours. Quantitative research approaches, such as surveys, are used to explain this kind of behaviour.The integration of both areas of research into one study is unique. However, technological advancement automatically generates new possibilities for data-driven research. In this research, traditional data collection techniques are combined with more innovative approaches. The first study that was conducted, presented in Chapters 6-8, is a survey to identify the factors affecting individual social behaviour in CoPs. The chapters 9,10 and $\mathrm{II}$, are a step further. The individual responses to the survey were-with the permission of the respondents-connected to their social behaviour in their CoPs. The online community software used in the cases of this research generate loads of web-based social interaction data. Typical examples of web-based social interaction data available through the online community software are posting messages, sharing other's posts, commenting on these posts, and chatting with another colleague. This data offers new and advanced possibilities to make meaningful statements about developments in the social behaviour of community members.

One of these possibilities is analysing the aggregation of direct and indirect social interactions between the users of the online community software in the online social networks, which results in options for creating social interaction networks (Atzmueller, 20 I4). These interactions are from the web-based social interaction data. Each social interaction type registered in the 
databases of the organisation gives rise to and clarifies the social interaction networks. Other options are based on for example text mining to analyse sentiments and trends (Cambria et al., 2014). However, in order to not only see the developments but also understand them, it has been decided that the web-based social interaction data will be enriched with the survey data used in chapters 6 to 8 . As indicated by Saarinen et al. (2015), purely focusing on the logged interactions and movements does not suffice when studying members' intentions, expectations, experiences.

This data mining approach presents the steps taken to achieve the purposes set for these studies. Data mining is a popular term often used interchangeably with the terms knowledge mining from data, knowledge extraction, data/pattern analysis, data archaeology, and data dredging (Han, Kamber, \& Pei, 20I2). In this dissertation, both the definition of Jiawei et al. (20I2) and the knowledge discovery process will be used. The definition proposed by jiawei et al. (20I2) is as follows: "Data mining is the process of discovering interesting patterns and knowledge from large amounts of data. The data sources can include databases, data warehouses, the Web, other information repositories, or data that are streamed into the system dynamically".

The underlying knowledge discovery process that determines the dissection of meaningful knowledge consists of the steps listed below (Figure 5.2). These steps are a combination of the CRISP-DM approach and the knowledge discovery process introduced by Han, Kamber, \& Pei (20I2).

I. The position of a CoP within an organisation contributes to its development.A clear business understanding is therefore essential for interpreting the results of the different studies. In Chapter 4, the primary cases in this dissertation are described based on interviews with the community managers.

2. Data gathering collects existing or newly created data. Existing data might be data from, for example social media, while newly created data might be data from survey research and interviews. Three types of data were collected in this dissertation: interview data, survey data, and web-based social interaction data.

3. The new or existing data are often full of noise, errors and further inconsistencies. The phase of data processing is therefore an important one and must take place before the actual data mining and further interpretations can be started. The data processing stage is a rather extensive one, which consists of data understanding, data cleaning, data integration, data selection, and data transformation.

a. Data understanding: forming an accurate picture of the data is important for interpreting the final analyses.

b. Data cleaning: detecting, correcting or removing noise, inconsistent or incomplete data from a data record set, table or database.

c. Data integration: merging and combining multiple data sources

d. Data selection: selecting data relevant for the purpose of specific studies.

e. Data transformation: transforming and consolidating the relevant data into forms suitable for mining.

4. One of the fundamental steps of the data mining process is the eponymous phase: data mining. Data mining represents the discovery of interesting patterns and knowledge from large amounts of (structured and unstructured) data.

5. In the subsequent step, the actual analyses are conducted. In the pattern evaluation step, the interpretation of the relevant patterns ultimately represents knowledge.

6. In the knowledge presentation step, the knowledge visualized in graphs, tables or trough textual means. 
7. Strategic decision-making represents the last step in this process. In this step, the results of the first steps should lead to a strategical decision to lead the CoP in a preferred direction.

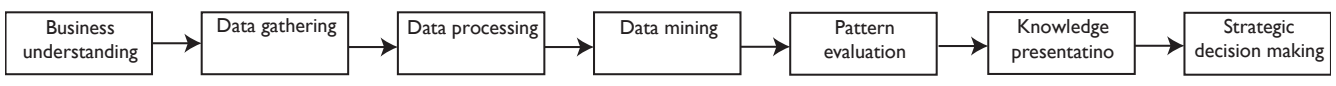

Figure 5.2. A typical data driven process

In the first area of the CMEM, the explanatory factors affecting an individual's social behaviour in CoPs are evaluated. In the second step, defined in the following paragraphs, the focus is on monitoring and evaluating the developments in CoPs. In the coming paragraphs, research methods that are more data-driven are introduced and described. Saarinen et al. (20I5) distinguish two methods for studying interactive application. First, we identified the sequences of interactions and behaviours by adopting an exploratory sequential data analysis (ESDA). This approach is largely a top-down approach in which the directions in the research are determined by predefined research questions. A second approach is using a mix of web-based social interaction data to analyse a variety of studies without a predefined thesis but with the central premise of identifying interesting behavioural patterns. The second perspective is used for the three studies described below in which the formulated research questions act only as a delineation to reduce the scope of the research. The behaviour patterns can be evaluated from various angles and positions. This research will examine the development phases in CoPs (study 2 ), the individual social network positions in CoPs (study 3), and the social interaction patterns in CoPs (study 4). Extensive descriptions of the research methods used are described in the corresponding chapters: Chapters 9,10 and II.

\subsection{I.Monitoring and evaluating developments in CoPs}

The direction of developments in a CoP is largely determined by human actions and decisions at the organisational level. This results in developments that may differ from one CoP to another. To make statements about these developments meaningful, it is important to look at behavioural patterns in a holistic way. In the study described in Chapter 9, the focus is on identifying common development patterns that exist in a CoP. Identifying these patterns contributes to our understanding of the different phases that each CoP will encounter during its lifetime. In this study, we use web-based social interaction data from the CoPs to holistically examine the developments of these CoPs. By taking a holistic view on the developments within CoPs and by looking at similar behavioural patterns within the CoPs participating in this study, we can make specific recommendations to monitor and possibly steer developments. With a data driven approach-based on activity measurement data from the first fourteen months of a CoP-we dive into the development of CoPs. Activity measurements represented in the management and usage of online community software were tracked and reported for the fourteen months. Changes during these months are analysed, and trends are evaluated (Gongla \& Rizzuto, 200I). The first 14 months represent the start-up phase. This start-up phase embodies the organisational and collective process of decision-making, implementation, adoption and use of the online community software. In these months, the CoP gradually gains a position within the organisation. Generally, some sub-communities in a CoP are active from the beginning, but the majority have a lead time of a few months before everyone recognizes the added value and acts accordingly.

The next three steps will be followed to obtain an overview of the changes during the startup phase: (I) identify and formalize the social behavioural development patterns in the CoPs, 
(2) identify community member roles in the CoPs based on their relatively most frequent types of social interaction and (3) analyse relationships between the community development patterns in the first step and the distribution of member types in the second step.An extensive description of this study is presented in Chapter 9.

\subsubsection{Evaluate individual social network positions in CoPs}

The level of social activity of an individual community member in a CoP is often a valid indicator for that community member's appreciation of the CoP. However, analysing these social interactions also indicates the member's position in the CoP, from central to peripheral to bridging. Consciously or unconsciously, people take up a certain position within a social network. In one moment, a member may take the lead, but in another moment that member may observe what is happening or connect people to each other. These positions can change over time, and the presence of these varying positions contributes to improved insight into the composition of CoPs. One of the most widely interpreted network positions is the member role (Doran, 20I5; Gleave, Welser, Lento, \& Smith, 2009). The persons with whom an individual interacts are associated with the other members' perceptions of the individual, his or her authority, and his or her networking and dissemination skills (Zaheer \& Bell, 2005).

In this study, web-based social interaction data are used to determine an individual's network position. These data provide insight into five types of social interaction that represent a direct relationship between two people: a comment on another community member's post, the recommendation of a post to another community member, the appreciation of the contribution of another community member, the following of the contributions of another person, and engaging with a chatting mechanism in which two persons send direct messages to each other. Social network analysis techniques are used to unravel the developments in each individual CoP. The social interactions that an individual establishes with other CoP members tell us something about the position of that individual. In the survey study-the email addresses from the participants were collected, and the web-based social interaction data of those community members who gave permission were connected to the survey data. In this dissertation, the combination of web-based social interaction data and survey data provide a unique insight into the reasoning behind certain activities within the online environments. Identifying individual social network positions is the first step and merging the individual network positions with the MOAC factors measured in the survey study is the second step. In contrast to the individual perspective (Chapter 6 to 8 ) whereby MOAC is further disaggregated into smaller explanatory parts (e.g. time, work autonomy), the chapters 10 and II use the motivation, opportunity, ability and communality as exogenous and endogenous variables related to the group behaviour. By splitting the web-based social interaction data into two periods, it is possible to include the time factor in the development of network positions. In addition, the model is also reversed, investigating the relationship between the different network positions (exogenous) and the MOAC factors (endogenous). This combination allows us to find possible driving factors for the position of an individual in his or her CoP.A more elaborate description of this study is presented in Chapter 10.

\subsubsection{Evaluate social interaction patterns in CoPs.}

The web-based social interaction data derived from the multiple cases provide insight into the development of the online CoPs on a general level and on the more specific individual level. There are numerous opportunities that come from meaningfully gathering knowledge from 
the large web-based social interaction data files collected and stored by the online community software. In the second area of the CMEM, the development phases in CoPs are determined (study 2), and in the third area of the CMEM, the individual network positions in CoPs are discussed (study 3). The last study focuses primarily on the identification of social interaction patterns. The emphasis is on investigating which patterns occur during a certain period in a CoP. In this study, the connection will be made between the results from the questionnaire and the web-based social interaction data with the same data-merging approach as in study 3 . One of the methods that is suitable for analysing social interaction patterns is exponential random graph modelling (ERGM).

This modelling approach is one of the accepted statistical modelling techniques for social networks (Wang, Pattison, \& Robins, 2013). Communication in CoPs is often not as straightforward as expected. These CoPs are organized in sub-communities with open communication within these sub-communities, which provides one-to-many interaction forms (Malinen, 20I5). The fact that several people may have read a message creates a great deal of bias. It is unknown who has read a message and who has not. To avoid ambiguity and confusion, 2-mode network graphs will be analysed, with an emphasis on analysing those social interaction patterns in which individuals exhibit similar social behaviour. This means that there is no direct relationship between two people, but there is (possibly) an indirect relationship. The direct relationship in a social network is between a person and a form of social interaction (e.g., posting a message or commenting on a message). Insight into these social interaction patterns enables community managers to approach specific participants in a CoP in order to further the strategical development of CoPs. An elaboration of this area of the CMEM is described in Chapter II.

\subsection{Summary}

In summary, each area in the CMEM approaches the developments in CoPs from a different angle. However, the variation also results in complementarity, as each individual study considers the 'development' phenomenon in a different way. Thus, the CMEM consists primarily of determining the factors that influence an individual's social behaviour and, in turn, the impact that the individual consciously or unconsciously has on group behaviour, which is the reason for incorporating the developments in social behaviour patterns and the impact of the individual through the different member types. The position that an individual has within the underlying network that forms the social basis of the CoP also has implications for the social composition of a CoP; additionally, the social interaction patterns reveal something about the background of social behaviour (individual and group) and form an important predictor for the directions of social behaviour. 

PART III:

INDIVIDUAL

SOCIAL

BEHAVIOUR IN

COMMUNITIES OF

PRACTICE
The MOAC-framework presented in Chapter 3 will be tested and evaluated in the Chapters 6,7 and 8. The generally accepted form from where most sociopsychological models start is from the individual focus area, where the spheres of influence of the individual are of primary importance. In this part of the explorative research, the factors in the MOACframework are divided into three types of factors: individual factors, organisational factors, and community factors. The research question central in this part of the thesis is: What individual, organisational and community factors affect the social behaviour of community members in a community of practice?

Chapter 6 focuses primarily on the individual factors that influence individual social behaviour in communities of practice. The exogenous variables tested in this chapter are: motivation to be informed, motivation to improve work performance, social reputation, subjective norms, and an individual's ability (ability to express, ability to contact \& digital skills) versus the endogenous variables: knowledge acquisition behaviour and the social interaction behaviour.

In Chapter 7, special attention is given to the organisational factors that affect an individual's social behaviour in communities of practice. The exogenous variables tested in this chapter are: time, work autonomy, and facilitating conditions versus the endogenous variables: knowledge acquisition behaviour and the social interaction behaviour.

The study in Chapter 8 is centred around the community factors with special attention to specific group variables. The exogenous variables tested in this chapter are: shared language, shared identity, and shared leadership versus the endogenous variables: knowledge acquisition behaviour and the social interaction behaviour.

Parts of this dissertation will be used for future scientific publications 



\section{Individual Factors Driving Social Behaviour in Communities of Practice}

\section{I. Introduction}

This chapter aims to unravel factors that affect an individual's social behaviour in a CoP. In the current knowledge-intensive economy, a professional's knowledge utilization is identified as a crucial resource for the successful development of organisations (Lau, Lee, \& Chung, 2019;Tsvyk \& Tsvyk, 2018). Optimizing these knowledge-intensive processes within an organisation has, for that reason, a fundamental place on the organisation's agenda. To realize this objective, most organisations mainly examine the potential offered by online communication networks such as CoPs to enable members to share knowledge and engage in continuing workplace learning and professional development. Successfully implementing CoPs within organisations has proven to be a challenge. The attention given to the development of this type of organisational learning is continuously increasing, but an adequate answer to factors that affect individual social behaviour is still missing. Ardichvili, Page, \& Wentling (2003) have taken a significant step towards the identification of factors that determine social behaviour in CoPs. These authors hit the nail on the head by showing that if individuals view knowledge as a public good belonging to the whole organisation, knowledge flows easily. However, the qualitative research of Ardichvili et al. (2003) just gives a preliminary indication of potential factors in a specific context; the intention of this study is to explore whether a more elaborated model can give a better explanation of individuals' social behaviours in communal environments.

Most studies focus on several concepts of the processes within CoPs. Some popular areas of research include knowledge distribution in virtual or physical environments (Ardichvili et al., 2003; Siemsen et al., 2008) and in specific contexts (Hara \& Hew, 2007). A substantial proportion of the studies focus on the intentions and motivations underlying knowledgesharing behaviours. This study goes beyond intention and aims to identify factors that determine individuals' practices within CoPs by conducting a quantitative study in four different organisations that already run a CoP. Since not all CoPs have the same performance of results, it is crucial to identify factors that enable or constrain organisational knowledge improvement processes. In our study, the scope of research is limited to CoPs that are mainly focused on mutual [or communal] interactions within the organisation in order to enhance the efficiency, effectiveness and innovative capacities of all stakeholders. Our goal is to take an initial step towards the development of a model to explain social behaviour in communal spaces such as CoPs. The MOAC model-introduced in Chapter 3-is used as an umbrella concept for structuring potentially stimulating factors that influence the social behaviour of individuals in CoPs. The original MOA model is by nature quite generic and therefore offers relatively little help in explaining social behaviour. This dissertation offers a first specification, elaboration and concretization of the existing MOA model. As the MOAC model consists of individual, organisational and community factors that all affect individual behaviour in CoPs, the model has been split accordingly. In this chapter, the focus is on the individual factors: motivation and ability. The sub-question formulated for the purpose of this study is as follows: What are the reasons, reports and perceptions of professionals about their knowledge acquisition and social- 
interaction behaviour that affect participating in Communities of Practice? In Chapter 7, the focus is on the organisational factors, while Chapter 8 consists of the community factors.

\subsection{Theoretical developments and hypotheses}

\subsection{Community of practice}

The definition of a CoP-introduced in Chapter 2-is used as a point of reference for the entire dissertation. The term CoP in this dissertation means: "a social network where people in an organisational context come together around a common topic, passion or interest and regularly interact on- and offline with a focus on knowledge management, innovation, learning and social networking". These social networks may be used for empathetic support but are more often used for common interest, information sharing and problem solving. They are characteristically easy to enter and leave, non-exclusive and have heterogeneous membership (Andrews, 2002; Lave \& Wenger, 1991; Wellman, 1997).

\subsubsection{Social behaviour}

Activity is a crucial factor for the success of CoPs (Malinen, 2015; Wenger \& Snyder, 2000). In traditional offline CoPs, many individuals feel social pressure from other attendees to communicate, primarily because face-to-face communication is a rich medium with a physical audience. In the online version, it is much simpler to passively participate without contributing relevant knowledge. The vast majority of individuals within online communities therefore play the well-known role of observer, while posters are in the absolute minority (Hurtubise, Rivard, Berbari, Héguy, \& Camden, 2017).

The difference between posters and observers is that posters make contributions to the community by sending messages, videos or other media, while observers stay silent most of the time. Sun, Rau, \& Ma (2014) follow the often referenced "90-9-I" principle, which states that on collaborative websites, such as that of an online community, $90 \%$ of the participants only read content, $9 \%$ of the participants edit or comment on content and $1 \%$ of the participants actively create new content. These numbers may differ in each community, but in fact, despite the great potential of CoPs, many online environments are used sporadically or are used intensively only by a small group of users.

Existing studies provide us with rich underpinnings to build a research model for examining continuing social behaviour in CoPs. Lampe, Wash,Velasquez, \& Ozkaya (2010) define one base measure of social behaviour as the creation of an account in a system.After creating an account in the system, one can decide to participate actively or to mainly consume content by reading posts, watching videos, or reading articles-all without contributing to the production of the resources they use. Hooff \& Ridder (2004) also made the distinction between knowledge collection and knowledge donation. Ultimately, all these different types of social behaviour can be classed under active and knowledge-acquiring behaviour, where social interaction behaviour consists of knowledge donation, social networking behaviour and knowledge acquisition behaviour, and that community members who predominantly show knowledge acquisition behaviour are mainly acquiring knowledge and information. These two types of social behaviour will be briefly outlined in the coming sections. 
Social interaction behaviour

Knowledge donation (or knowledge sharing) occurs when an individual communicates to others about his or her personal intellectual capital (Van den Hooff \& de Leeuw van Weenen, 2004). Sharing knowledge with others in a CoP is a crucial activity for the success of a CoP.A CoP without members who contribute their knowledge and skills to it will quickly decrease in relevance for its participants. The effectiveness of knowledge sharing largely depends on the context of the activity. According to Fadel \& Durcikova (20I4, p.3606), knowledge sharing is "most profitable among those who engage in similar work activities for which a common knowledge base is required". Meanwhile, a significant number of studies have been carried out on factors contributing to sharing knowledge (Bigné et al., 20I5; Siemsen et al., 2008).

A second form of activity - which is being increasingly seen in CoPs-is networking activity (Bock, Robert, \& Kim, 2005; Pan et al., 20I5). The networked nature of a CoP ensures that participants increasingly use these platforms to search for peers or professionals with a distinctive form of expertise that the seeker lacks. Baker \& Beames (2016) argue that CoPs are increasingly used to connect with others to make working processes more efficient. For example, when a community member needs a professional within a certain field of expertise, that member can use a directory with a description of the expertise of the community members to find and connect with relevant persons. Transforming this 'just identifying others' into 'professional relationships' may help community members acquire potentially valuable resources and reliable support via this professional network (Tseng \& Kuo, 20I4).

\section{Knowledge acquisition behaviour}

Generally speaking, the majority of community members might not actively contribute to the knowledge base of a CoP but do still profit from the CoP by, for example, identifying who is working on new ideas or what procedure to follow in a certain situation (Baker \& Beames, 2016). These individuals are often characterized as observers who primarily benefit from the efforts of others. Therefore, the main focus of community managers is to encourage these non-visible participants to participate in a more active way, thus rendering these invisible users more visible in the online environment (Malinen, 20I5). Many community members can be characterized as observers. A lack of self-confidence or knowledge can be the basis for taking this position, but there are undoubtedly other motives for less social interaction behaviour. Knowledge donation and knowledge acquisition are identified as hallmarks of CoPs (Pan et al., 2015).

\subsubsection{Motivation}

The way an individual act largely depends on the individual's motivation to exhibit certain behaviour. Many scientists have analysed the motivation to participate from two perspectives: the level of motivation (Siemsen et al., 2008) and the type of motivation (Lampe et al., 20l0). By analysing the level of motivation, it is crucial to identify factors that affect the weight of this motivation. Several social theories have been developed to define the second form of motivation: the type of motivation. Some examples are the theory of reasoned action (e.g., Fishbein \& Ajzen, 1975; Fisher, Fisher, \& Harman, 2003), the theory of planned behaviour (e.g., Ajzen, 1991; Godin \& Kok, 1996), and social-cognitive theory (e.g., Wood \& Bandura, 1989). The uses and gratifications (U\&G) theory (Katz, Blumler \& Gurevitch, 1973) is a theory that assumes that people have options and free will to act in a certain way. In the context of the U\&G, individuals can make specific decisions about which media to use and when to use it. This theory argues that audience members are active; they act in the way that provides them with 
the individual gratifications they seek. In this part of the research, the motivation is interpreted as a type of motivation, while in the last two empirical chapters (I0 \& II) the level of motivation is used.

Ardichvili et al. (2003) conducted an exploratory, qualitative study on the motivations and barriers to professional social behaviour in virtual knowledge-sharing CoPs at the US-based multinational company Caterpillar. In their research, a distinction is made among the motivations to contribute with knowledge (Bock et al., 2005), gather knowledge (Lee \& Ma, 20I2), or improve internal and external collaboration (Lancini, 20I5), which might be facilitated in a CoPs. The authors also assume that people participate in CoPs out of their own free will and without pressure from a hierarchically higher-ranked person. However, in many CoPs, one motivation for an individual's social behaviour is the perceived social pressure from direct colleagues (Scarbrough, 2003) or higher-ranked colleagues/management (Lewis, Agarwal \& Sambamurthy, 2003). Knowledge is increasingly seen as a public good, belonging not to individuals but to the whole organisation (Stiglitz, 2015). This could be a plausible reason why individuals actively participating in knowledge-intensive initiatives within a CoP.When communities reach the stage where people participate out of altruistic grounds, community behaviour is motivated by moral obligation and community interest, not by a narrow self-interest (Wasko \& Faraj, 2005).Another motivation often introduced comes from the study of Ardichvili et al. (2003) and is associated with various self-based considerations, such as individuals expressing themselves as experts or helping others as experts. Nevertheless, there is also a substantial proportion of community members who participate to improve their own work performance (Han, Zheng, \& Xu, 2007; Kulkarni, Ravindran, \& Freeze, 2007), which can be aimed at organizing their work more efficiently or effectively or improving their work activities. In this dissertation, four reasons are identified as important motivations for social behaviour in a CoP: being informed, improving work performance, improving social reputation, and subjective norm.

\section{Informing}

People joining a CoP often do this to fulfil the need to gain validation of their own professional practice or just to engage in knowledge building in order to stay informed about current technology, best practices and other relevant information (Hara \& Hew, 2007). Lee \& Ma (20I2) used the uses and gratifications theory to identify the three primary motivations relevant in news sharing within social media environments. These three motivations are information seeking, socializing and status seeking. Socializing and status seeking are two fundamental components of the concept of work performance, as discussed in the upcoming paragraphs. Information seeking is one of the essential components of the motivation to be informed. Information seeking refers to the extent to which information and knowledge shared in CoPs offer users relevant and timely knowledge or information (Lee \& Ma, 20I2). The phenomenon of the observer arises from the urge to be informed about matters that are important for carrying out work activities without the observer making any active contributions himself. These observers rarely contribute to the CoP by posting knowledge and information but gain insight from reading discussions, information, and other resources. According to Hurtubise, Rivard, Berbari, Héguy, \& Camden (2017), as many as $60-90 \%$ of CoP users can be defined as observers. One of the primary motivations for these individuals is the opportunity to read content from others. By reading the contributions of others-who are often identified as 'experts' - to the learning capacity of the community members, an increasing validation of practices will be realized. However, being informed can also be a motivation for the community members who are more actively participating. The motivation to be informed is a rather passive method of social behaviour. It is expected that community members who are motivated by the 
opportunity to be informed are less likely to actively contribute to the CoP, and the logical consequence is expressed in the following hypotheses:

HIa: The motivation to be informed has a positive influence on the level of knowledge acquisition behaviour among the CoP community members.

HIb: The motivation to be informed has a positive influence on the level of social interaction behaviour among the CoP community members.

\section{Work performance}

IT applications are often introduced in organisations to improve work efficiency. Improving work efficiency can be seen as a mutual benefit for both organisations and professionals. Especially when working on a certain case, a just-on-time solution that comes from asking one's peers might be beneficial.When considering work efficiency, many academics make associations to knowledge management applications (Chen, Chen, 2009; Du Plessis, 2005). By creating an adequate knowledge management strategy, organisations will benefit from the improved work efficiency of their employees. Du Plessis (2005) exemplifies this work efficiency as increased organisational capacity through five examples: improved customer service, improved resolutions of business problems, increased productivity, and improved leveraging of corporate and individual knowledge.

These knowledge management processes are increasingly embedded in CoPs. The technologies developed as a foundation for these CoPs are not only developed to improve knowledge management in organisations but also to improve the work efficiency of community members. Members must be able to access a large source of tacit (e.g., other community members) and explicit (e.g., documents, news, blogs) knowledge and information easily to adequately address certain issues (Lee \& Ma, 20I2). The idea behind a CoP is to improve practice without reinventing the wheel repeatedly. The interactive characteristics of a CoP contribute to the fast and adequate identification of solutions to issues that are also experienced by others or have already been solved by others. In addition, a CoP also offers opportunities for improving existing practices or creating new practices that might be beneficial for peers.

In addition to improving work efficiency, another motivation which is closely related to the pursue to improve work performance is the desire to improve work quality (Kulkarni et al., 2007) in order to be competitive as an organisation or to remain capable enough as an employee. Collaboration, continuous learning and knowledge management are extremely important, as these add to the quality of the work within the organisation. In particular, sharing personal advice improves work quality and signals competence (Haas \& Hansen, 2007). In their research, work quality is used as a synonym of "the extent to which the output of a task meets or exceeds the expectations of those who receive it or use it" (Haas \& Hansen, 2017, p. I I37). In knowledge management and business improvement, the emphasis is increasingly on social learning in which the personal dialogue between individuals contributes to the improvement and innovation of business and individual practices.

In general, work quality is often operationalized more broadly than only the labour output. Work quality is often defined as the form of perceived usefulness of sharing knowledge, which might be termed "the subjective evaluation of the extent to which the person believes that contributing to and using available and knowledge sharing capabilities existing within the organisation improves his or her job performance, productivity, effectiveness, ease of doing the job, and so on" (Kulkarni et al., 2007, p. 3 I5). Bock et al., (2005) found a positive significant 
relationship between the perceived usefulness of electronic knowledge repositories and the actual use of these repositories. Ultimately, these processes should lead to the continuous improvement of individual and collective practices in organisations.

This results in the following two hypotheses:

H2a: The motivation to improve work performance positively affects the level of knowledge acquisition behaviour among CoP community members.

H2b: The motivation to improve work performance positively affects the level of social interaction behaviour among CoP community members.

\section{Social reputation}

Enhancing one's social reputation among colleagues_or status seeking-is one of the extrinsic motivations for an individual to create or share knowledge in a CoP (Kulkarni et al., 2007; Lee, Reinicke, Sarkar, \& Anderson, 20I5). According to Lee \& Ma (20I2), a distinction must be made between one's feeling of importance and one's desire to be admired by one's peers. In knowledge management situations, an individual often seeks recognition by his or her peers; individuals want to present themselves as highly skilled, experienced and knowledgeable professionals (Wasko \& Faraj, 2005). Park et al. (2007) found evidence for this by showing a positive relationship between status seeking and social outcomes such as public social behaviour. The study of Lee, Reinicke, Sarkar, \& Anderson (20I5) yields the same result by showing a positive relationship between reputation and behavioural intensity. The role of the motivation to improve one's social reputation in an individual's social behaviour results in the following hypothesis:

H3: The motivation to improve one's social reputation positively affects the level of social interaction behaviour among CoP community members.

\section{Subjective norm}

A last - less voluntary - motivation for professionals to participate in CoPs is presented as the subjective norm in the organisation. Subjective norm are often bracketed together with the degree of social pressures that exists in a CoP. Ajzen \& Albarracín (2007, p.5) defines subjective norms as the "perceived social pressure to perform or not perform the behaviour". It is the thinking of others that activates our desire to act (Pavlou \& Fygenson, 2006). Applied to behaviour in CoPs, subjective norm reflects "participants perceptions of whether the behaviour is accepted, encouraged and implemented by the participant's circle of influence" (Chen et al., 2009). Literature suggests a positive relationship between subjective norm and intended behaviour. This implies that when they see that colleagues actively contribute in a CoP, they themselves also tend to show the same behaviour. The amount of social pressure comes mainly from organisation management and direct colleagues (Bagozzi et al., 2007; Hung, Lai, \& Chou, 20I5). However, the 90-9-I rule (Brandtzaeg \& Heim, 20II) show that the majority of community members are observers of the behaviour of peers in the CoPs. Their mode of activity mainly focuses on acquiring knowledge and information and applying it in their practice without adding knowledge and information to the CoP. Social pressure can also put pressure on people to show more active types of social behaviour that are desired (Harris, 20I5). The result of this is that participants generally participate due to social pressure, without being actively involved in the other more active community processes. This results in the following hypotheses:

H4a: The subjective norm (social pressure) positively affects the level of knowledge acquisition behaviour by the community members 
H4b: The subjective norm (social pressure) positively affects the level of social interaction behaviour by the community members

\subsubsection{Ability}

Bigné et al. (20I5) has found significant evidence that an individual's ability to act in a certain manner is an influencer of that individual's social behaviour. The authors argue that when a user feels he/she has the capacity to exchange information about - in this case - airline services, chances of participation in the networks arise. An individual's ability can be seen as the talent, skills, or proficiency required to commit an action (Hughes, 2007; Turner \& Pennington, 20I5). One term that is often used synonymously with the ability to express oneself is knowledge self-efficacy. Knowledge self-efficacy refers to one's confidence in one's ability to provide knowledge that is valuable to others (Hung, Lai \& Chou, 20I5). The higher an individual's sense of knowledge self-efficacy, the more likely it is for an individual to increase his or her use of a knowledge management system (Bock et al., 2006). One action within online collaborative environments is knowledge management. Wick (2000) has observed that knowledge has limited value, since it becomes obsolete very quickly. This makes it essential for an organisation to recognize knowledge as an asset and knowledge management as a skill. However, Wick (2000) also argues that knowledge in itself is not a very valuable resource; it is an individual organisation member's ability to generate knowledge and innovate by using that knowledge. Venkatesh et al. (2003) generally confirm this statement; these researchers emphasize that individuals need digital skills to act in a meaningful way in contemporary CoPs, which increasingly occur in online environments.

The research from Siemsen, Roth, \& Balasubramanian (2008) confirms Wick's (2000) statement, since the former have proven the relevance of an individual's ability in the decision to share knowledge within CoPs. If an individual has a strong sense of knowledge self-efficacy, he or she will have no problem increasing his or her use of a knowledge management system (Bock, Robert, \& Kim, 2005). Turner \& Pennington (2015) found a small, significant and negative association between ability and knowledge sharing, and according to Bandura (1982), it is highly rational that when individuals lack a certain level of confidence in their ability to share knowledge, the chance that they perform in a certain manner is also reduced. Factors that determine an individual's ability are identified by Cadiz, Sawyer, \& Griffith (2009), Siemsen et al. (2008) and Turner \& Pennington (20I5) as the ability to express oneself and social networking skills. People need skills to express themselves and share their knowledge and skills, but in contemporary networked society, the ability to make (online) contact has also become crucial. These two factors and digital skills define an individual's ability to act.

\section{Perceived ability to express}

'An individual who wants to participate in a CoP must be able to transfer knowledge to his or her peers': this is a common fundamental assumption when speaking about an individual's ability. However, meaningful social behaviour in CoPs is not synonymous with the ability to transfer knowledge; there are many other capacities that can also add great value to a CoP. Conveying a message is more than only knowledge sharing, it is the ability to express oneself within a CoP that contributes to one's value addition in a CoP.

Bettiga et al. (2018) found a strong relationship between an individual's ability to express themselves in a community for virtual co-creation with the willingness to co-create. Most definitions used to describe an individual's ability are related to one's skills and proficiencies 
that are required to share knowledge with co-workers (Macinnis et al., |99|; Rothschild, 1999; Siemsen et al., 2008) Turner \& Pennington (2015) were some of the first researchers to examine social behaviour in a CoP by applying the MOA model and found evidence that sharing knowledge is also about the confidence to ask questions, the capacity to comment in discussions and the ability to convey emotions. These types of social interaction behaviour often also trigger a chain reaction. The willingness to participate increases when something is perceived as having immediate value, such as participating in engaging discussions, forming new working relationships, having the ability to share one's views, finding solutions to one's problems, having the opportunity to see what others are doing and discovering tools, documents or techniques that one can use in one's own work (Pyrko et al., 2017). All of these competencies are interconnected, and together, they represent one's ability to add value to a CoP.

H5a: An individual's perceived ability to express positively affects the level of knowledge acquisition behaviour among the community members in a CoP.

H5b: An individual's perceived ability to express him or herself positively affects the level of social interaction behaviour among the community members in a CoP.

\section{Perceived ability to contact}

In modern society, an individual's networking capacity is a fundamental skill in the development of professional careers, supporting the individual's growth, learning and opportunities. Meeting new contacts relevant in performing one's job becomes easier in online environments. Contacting colleagues from other departments in one's organisation or even beyond the boundaries of one's organisation is sometimes a large step for individuals. Rajagopal, Joosten-Ten Brinke, Van Bruggen \& Sloep (20I2) recognize the social networking skills as key skills in the life-long learning of individuals; these skills form an individual's capacity to connect with relevant others by, for example, professional networking.

Collaboration across the boundaries of teams, departments and organisations not only consists of 'being able to express oneself', but, above all, being able to connect and being able to identify relevant-yet previously unknown - contacts. By improving social networking skills and creating a relevant social network, the possibilities to collaborate also increase. Homans (1958) argues that social interactions are fundamental for building and continuing social relationships. A lack of openness and accessibility with regard to giving help and advice when asked by others has negative consequences for the number of social interactions and amount of appreciation from others (Kraut \& Resnick, 20II), and conversely, when the frequency of social interactions increases, co-workers' liking for one another also increases.

As stated by Cadiz, Sawyer, \& Griffith (2009), it is unlikely that social interaction would occur between community members without open communication. A community without social interaction would result in a dissolving community. Baran \& Cagiltay (2010) emphasize the relevance of self-confidence as an important factor in online environments. Another factor also introduced in the study of Baran \& Cagiltay $(2010)$ is that sociable community members who easily adapt to their environment more frequently communicate with others. People who have not meet each other before and who meet each other for the first time online are less likely to start an interaction with each other (Vonderwell, 2003).

Backstrom, Huttenlocher, \& Kleinberg (2006) found evidence that the likelihood of community members joining a CoP increased with the number of current members of the community they were linked to. This implies that the presence of already-known colleagues can influence 
individuals when they must decide on their own social behaviour. It is expected that an individual's social networking skills contribute to actual social behaviour in CoPs.

H6a: An individual's perceived ability to contact positively affects the level of knowledge acquisition behaviour among the community members in a CoP.

H6b: An individual's perceived ability to contact positively affects the level of social interaction behaviour among the community members in a CoP.

\section{Perceived digital skills}

Traditional, predominantly physically oriented CoPs required only soft skills to act in a meaningful manner. The transformation of the CoPs into mixed media CoPs requires new-more digitally oriented-skills as well (Van Dijk, 20I2). Deursen, Van Dijk, \& Peters (20II) introduce two categories to describe the digital skills: medium-related skills and content-related skills. These two categories can be subdivided into smaller units, media-related skills within operational skills (i.e., operating digital media) and within formal skills (i.e., web browsing, navigating). Contentrelated skills include information skills (i.e., valid search, selection and evaluation of digital media content), communication skills (i.e., effective exchange of meaning), content-creation skills (i.e., create acceptable contributions in digital media), and strategic skills (i.e., use digital media for a purpose).

By facilitating the conditions for optimal social behaviour in a CoP, an organisation stimulates its employees to become active community members. However, digital skills are necessary to make meaningful use of the technological component of the facilitating conditions. Van Dijk (2006) was one of the first academics with a special interest in digital skills in our society and emphasized their future relevance in our modern networked society. More than ten years later, these predictions have been fully realized. The digitization of our labour market has ensured that, in addition to the knowledge and competencies that are part of a specific job, digital skills should now be prerequisite skills. The European Commission has recognized this by including digital skills in the 'new skills agenda' (European Commission, 2016). It is a plausible assumption that a lack of perceived digital skills might have a significant impact on actual social behaviour in CoPs. Limited perceived digital skills might result in a decrease in confidence to act. Since digital skills might influence all types of social behaviour in a digital environment, the following hypotheses are formulated:

H7a: An individual's perceived digital skills positively affect the level of knowledge acquisition behaviour among the community members in a CoP.

H7b: An individual's perceived digital skills positively affect the level of social interaction behaviour among the community members in a CoP 


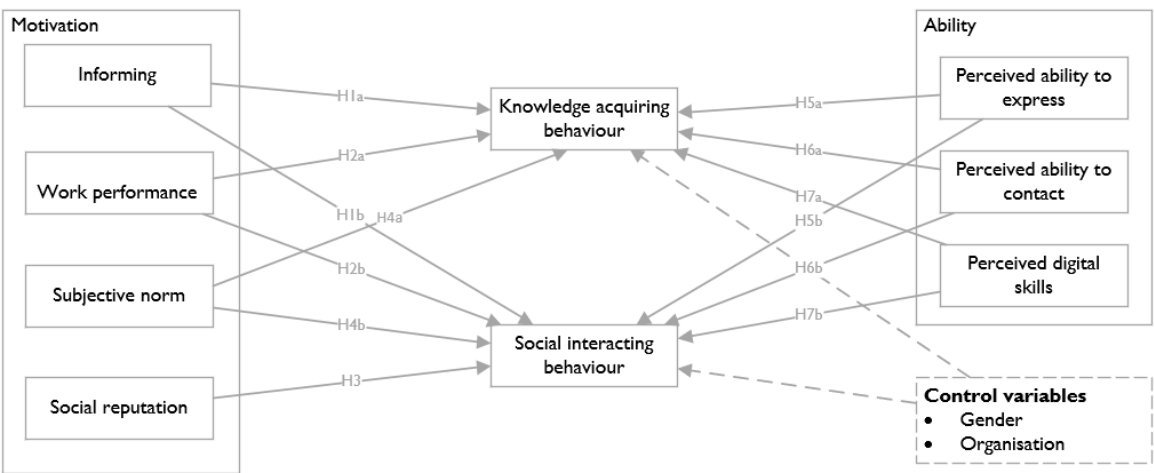

Figure 6.I. Research Model Individual Perspective

\subsection{Methods}

To validate the conceptual MOAC model, we conducted an extensive online survey in four organisations. The online survey-which formed the basis for the studies in chapters 6 through 8 - gave us a better understanding of the diagnostic and prescriptive community statistics, an important part of the community monitoring and evaluation methodology (CMEM), as described in Chapter 5. By gaining insight into possible drivers and barriers to social behaviour, we can develop a better view of social behaviour in CoPs.

The organisations in this dissertation all use the same social technology, and the manufacturer of this social technology brought us into contact with the four context-exogenous organisations but did not have any influence in the remainder of the study. The four Dutch organisations that took part in this research were a financial service organisation, a large hospital, a youth care institution and an organisation that provides support for people with disabilities. A detailed description of these cases is available in Chapter 4 of this dissertation.

\subsection{Sample}

The survey was pre-tested with ten practitioners with different professional backgrounds. The main objective of the pre-test was to identify the ambiguities in the questionnaire. Unclearly formulated questions were reformulated to ensure that the questionnaire was in line with the target audience. The final version of the questionnaire was then sent to the four organisations, who distributed it through their own communication channels. In this study, a convenient sample of 221 community members of four organisations fully completed the survey. The respondents were invited to participate following a self-selection sampling method, and the respondents were recruited by email, newsletters and specific messages within the CoPs. The data collection was between December 2015 and April 2016. In total, 22I respondents fully completed the questionnaire, resulting in response rates ranging from $10 \%$ to $16 \%$ (Table 6.1 ), which is low but not unusual for this form of research in which rather long surveys are used and accessing respondents is difficult (Frohlich, 2002). The response ratio was measured by dividing the number of respondents by the number of potential respondents, the latter of which was based on the number of registered members in the community at the start of the survey. Additionally, the number of inactive or sporadic users in the CoPs was rather large, which might have reduced the response rate as well. Furthermore, the results are not equally distributed 
with respect to gender. One important reason is that the health care domain is dominated by women and the financial sector is dominated by men (Evans \& Steptoe, 2002).

Table 6.I

Sample characteristics

\begin{tabular}{|c|c|c|c|c|c|}
\hline & Overall & $\begin{array}{l}\text { Financial } \\
\text { service } \\
\text { organisation }\end{array}$ & $\begin{array}{l}\text { Large } \\
\text { hospital }\end{array}$ & $\begin{array}{l}\text { Youth care } \\
\text { Institution }\end{array}$ & $\begin{array}{l}\text { Organisation } \\
\text { for the care } \\
\text { of disabled } \\
\text { people }\end{array}$ \\
\hline$N^{\prime}$ & 221 & $\begin{array}{l}42 / 270 \\
(15.5 \%)\end{array}$ & $\begin{array}{l}90 / 818 \\
(11.0 \%)\end{array}$ & $\begin{array}{l}56 / 538 \\
(10.4 \%)\end{array}$ & $\begin{array}{l}33 / 273 \\
(12.1 \%)\end{array}$ \\
\hline$\%$ women & 72.9 & 40.5 & 80.0 & 76.8 & 87.9 \\
\hline \multicolumn{6}{|l|}{ Age (years) } \\
\hline$<25$ & $3.6 \%$ & $4.8 \%$ & $4.4 \%$ & $3.6 \%$ & $0.0 \%$ \\
\hline $25-35$ & $26.7 \%$ & $19.0 \%$ & $30.0 \%$ & $25.0 \%$ & $30.3 \%$ \\
\hline $36-45$ & $28.5 \%$ & $42.9 \%$ & $23.3 \%$ & $30.4 \%$ & $21.2 \%$ \\
\hline $46-55$ & $30.3 \%$ & $26.2 \%$ & $32.2 \%$ & $26.8 \%$ & $36.4 \%$ \\
\hline $56-65$ & $10.9 \%$ & $7.1 \%$ & $10.0 \%$ & $14.3 \%$ & $12.1 \%$ \\
\hline \multicolumn{6}{|l|}{$\begin{array}{l}\text { Experience } \\
\text { (years) }\end{array}$} \\
\hline$<1$ & $10.9 \%$ & $19.0 \%$ & $7.9 \%$ & $10.7 \%$ & $9.1 \%$ \\
\hline $1-5$ & $36.4 \%$ & $33.3 \%$ & $33.7 \%$ & $44.6 \%$ & $33.3 \%$ \\
\hline $6-10$ & $23.6 \%$ & $31.0 \%$ & $18.0 \%$ & $23.2 \%$ & $30.3 \%$ \\
\hline$>10$ & $29.1 \%$ & $16.7 \%$ & $40.4 \%$ & $21.4 \%$ & $27.3 \%$ \\
\hline
\end{tabular}

\subsubsection{Instrument}

The instrument used for this study to assess the individual factors that affect an individual's social behaviour in a CoP was part of a larger study that also addressed the organisational (Chapter 7) and community factors (Chapter 8 and onwards). Data in this study were collected through a questionnaire with a total of 36 items, which were divided into seven constructs. A seven-point Likert scale ranging from strongly disagree to strongly agree was used to measure the independent variables. The independent variables used in this study are framed as motivation and ability, derived from the MOAC model. The extended literature reviewdescribed in the first part of this dissertation-has shown that professionals in a knowledgeintensive environment have multiple motivations to participate in that environment.A subset of these motivations is the motivation to be informed (informing), the motivation to improve their work performance, the motivation to improve their social reputation, and the subjective norms of the organisation. These motivations form reasons to act in a certain manner within CoPs. The underlying construct introduced in the MOAC framework as the body of an individual's ability

' $\mathrm{N}$ is the number of responses usable for analysis (first number) and the number of active users registered in the online platform at the moment the questionnaire was distributed (second number). The response rate is given in parentheses. 
is comparable to the self-efficacy of individuals. These variables, which cover the individual's perceptions, are perceived ability to express, perceived ability to contact, and perceived digital skills. The dependent variables that cover the individual's social behaviour are knowledge acquisition behaviour and social interaction behaviour. The scale used to measure the dependent variables is a 6-point scale that measures the intensity of social behaviour ranging from never to multiple times a day. The scales used in this survey can be found in Appendix 6.I.

\subsection{Analysis and findings}

\subsection{Variables and measures}

In total, 24I community members in the organisations completed the survey. The data were loaded into IBM SPSS Statistics 25.0. Subsequently, negative formulated questions were recoded, and data were screened for missing values. Cases with missing values were removed from the dataset, which reduced our dataset from 24I cases to 221 cases. Outliers in the dataset have been included since social behaviour in digital collaborative environments such as CoPs often results in different types of users, with active preachers of success at one end and a more critical group at the other end. Thus, individual use and, consequently, perception can differ significantly. Additionally, the descriptive statistics shown in Table 6.I were calculated.

We subsequently loaded this final dataset into SmartPLS 3.0, a software package specifically designed for partial least square structural equation modelling (PLS-SEM), which is a technique to test exploratory models with a skewed distribution. The level of activity in the CoP is, as expected, rather skewed due to the varying levels of social activity.

\subsubsection{Data Analysis and Results}

In the first part of the statistical analysis, we used SPSS version 25 to analyse the descriptive statistics for this research. In Chapter 3, we define the variables used in this study. The measurement items used for each variable and the foundational papers from which the measures were taken are shown in Appendix 6.I and the mean scores on the constructs are described in Table 6.2. The conceptual model used in this research was adapted from prior studies. All items in the survey used to measure the theoretical variables were derived from an extensive review of the present literature.

Partial least squares equation modelling (PLS-SEM) is a modelling approach with no assumptions about data distribution, and where no normal distribution is needed. This makes it a good alternative for covariance-based structural equation modelling (CB-SEM), which expects normally distributed datasets. The difference between CB-SEM and PLS-SEM is fairly straightforward. When the research objective is theory testing and confirmatory in nature, the more suitable test is CB-SEM. If the objective is more predictive and theory developing, the PLS-SEM approach is the better option (Hair et al., 20I I). In the case of this exploratory research, it was a logical decision to select the PLS-SEM option, since sample sizes are small and the research objective is exploratory in nature. Hair et al. (20I I) argue that the sample size in PLS-SEM must follow a 'I0-times rule'; this rule builds on the assumption that the sample size should be greater than 10 times the maximum number of inner or outer model links, which, with twelve links and 221 respondents, is more than adequately guaranteed. CB-SEM expectsto a large extent - a strong theoretical foundation for the model; PLS-SEM is also based on theoretical foundations, but its primary goal is to predict the behaviour of the relationship among constructs and to explore the underlying theoretical concept. In the research presented 
in this study, the foundation is exploratory in nature and aims to identify relevant drivers for social behaviour in CoPs. In the coming paragraphs, we created a partial least squares (PLS) model to test the research hypotheses. The first step is to estimate the measurement model to ensure that the constructs are reliable and valid. The second step is to examine the structural relationships in the model that result in the structural model.

Prior to developing the measurement model, it is necessary to assess possible common method variance (CMV). Method variance is the variance that is determined more by the method than by the construct of interest, where the method refers to the form of measurement (e.g., scale type, response format, general context) (Fiske, 1982). Testing for the presence of CMV was performed using Harman's single-factor test (Podsakoff, Mackenzie, Lee, \& Podsakoff, 2003). In this approach, all variables are tested by an exploratory factor analysis without rotation. The common method bias can then be found by identifying the emergence of a single factor or a factor that explains more than $50 \%$ of the total variance. The score in this test is $35.9 \%$, which is well below this $50 \%$; thus, no general factor is present. Therefore, we assume that the common method variance is not of great concern for this study and thus it is unlikely that CMV bias the interpretation of our results.

\subsubsection{Measurement model}

The measurement model created for the purpose of this study started with the four independent variables representing an individual's motivation, the three independent variables measuring an individual's ability to perform, and the two dependent variables representing the level of social behaviour in a CoP.The three factors that were initially expected to represent different forms of ability were ultimately found to be insufficiently distinctive in the factor analysis (outer loadings); removing some of the underlying constructs would result in a very small scale to measure ability, which led us to create one compound variable: an individual's perceived ability. Thus, due to the interrelatedness of these variables, we have decided to merge these skills into one factor: ability. The compound hypotheses that follows are as follows:

H8a: An individual's perceived ability positively affects the level of knowledge acquisition behaviour among the community members in a CoP.

H8b: An individual's perceived ability positively affects the level of social interaction behaviour among the community members in a CoP.

The results described in Table 6.2 have been tested by means of PLS-SEM. Hair, Ringle, \& Sarstedt (20I I) argue that a PLS-SEM analysis generally consists of two stages: the assessment of the measurement model and the evaluation of the structural model. Central to this paragraph is the assessment of the measurement model. The assessment of the reflective measurement model mainly aims to evaluate the reliability and validity of the model. The properties of the measurement model are examined by the indicator reliability, convergent validity, internal consistency reliability of measures, and discriminant validity.

Final loadings and cross-loadings for the individual constructs are presented in Appendix 6.2. All items score well above the threshold of 0.4 (Hulland, 1999) recommended for exploratory research and in most cases even above the desired 0.7 (Kwong-Kay Wong, 20I3). The convergent validity of the model shows that each latent variable's average variance extracted (AVE) is above the acceptable threshold of 0.5 (Bagozzi \& Yi, 1988). This implies that the items suggested to measure the construct are indeed related. As for the internal consistency reliability of the 
measures - shown by using composite reliability - all scores well above the required 0.6 , which is acceptable for exploratory studies (Hair et al., 20 I I). The composite reliability of the measures is comparable to Cronbach's alpha; however, the former does not assume that all indicators are equally reliable, which makes it more useful for PLS-SEM. The results of the convergent validity and the internal consistency reliability of the measures can be found in Table 6.2.

Table 6.2

Constructs, variables (codes), sources, number of items, composite reliability, mean, standard deviation, and average variance extracted (AVE).

\begin{tabular}{|c|c|c|c|c|c|c|}
\hline Construct & Variable & Source & Items & $\begin{array}{l}\text { Composite } \\
\text { reliability }\end{array}$ & $\begin{array}{l}\text { Mean } \\
\& \text { SD }\end{array}$ & AVE \\
\hline \multirow[t]{2}{*}{$\begin{array}{l}\text { Social } \\
\text { behaviour }\end{array}$} & $\begin{array}{l}\text { Social } \\
\text { interaction } \\
\text { behaviour }\end{array}$ & $\begin{array}{l}\text { Millen, Fontaine, \& } \\
\text { Miller (2002) }\end{array}$ & 5 & 0.906 & $\begin{array}{l}2.13 \\
(0.96)\end{array}$ & 0.66 \\
\hline & $\begin{array}{l}\text { Knowledge } \\
\text { acquisition } \\
\text { behaviour }\end{array}$ & $\begin{array}{l}\text { Bock, Orces, Kim, } \\
\text { \& Lee }(2005)\end{array}$ & 2 & 0.800 & $\begin{array}{l}3.13 \\
(1.11)\end{array}$ & 0,67 \\
\hline \multirow[t]{4}{*}{ Motivation } & $\begin{array}{l}\text { Informing } \\
\text { (INF) }\end{array}$ & $\begin{array}{l}\text { Hara \& Hew } \\
(2007) ; \text { Lee \& Ma } \\
(2012)\end{array}$ & 6 & 0.918 & $\begin{array}{l}4.77 \\
(1.34)\end{array}$ & 0.65 \\
\hline & $\begin{array}{l}\text { Work } \\
\text { performance } \\
\text { (WP) }\end{array}$ & $\begin{array}{l}\text { Bock et al. (2005); } \\
\text { Han, Zheng, \& Xu } \\
\text { (2007); Kulkarni, } \\
\text { Ravindran, \& } \\
\text { Freeze (2007) }\end{array}$ & II & 0.960 & $\begin{array}{l}3,98 \\
(1,44)\end{array}$ & 0.71 \\
\hline & $\begin{array}{l}\text { Social } \\
\text { reputation } \\
\text { (SR) }\end{array}$ & Lee \& Ma (20I2) & I & 1,000 & $\begin{array}{l}3.40 \\
(1.70)\end{array}$ & 1.00 \\
\hline & $\begin{array}{l}\text { Subjective } \\
\text { norm (SN) }\end{array}$ & $\begin{array}{l}\text { Lewis, Agarwal } \\
\text { \& Sambamurthy } \\
\text { (2003); } \\
\text { Scarbrough, } \\
\text { (2003) }\end{array}$ & 2 & 0.892 & $\begin{array}{l}4.15 \\
(1.62)\end{array}$ & 0.81 \\
\hline Ability & Ability (AB) & $\begin{array}{l}\text { Siemsen et al. } \\
\text { (2008); Turner } \\
\text { \& Pennington } \\
\text { (20I5); Cadiz et al. } \\
\text { (2009);Venkatesh } \\
\text { et al. (2003) }\end{array}$ & 9 & 0.931 & $\begin{array}{l}5.44 \\
(1.03)\end{array}$ & 0.60 \\
\hline
\end{tabular}


Table 6.3 shows the discriminant validity in the measurement model. The discriminant validity is measured by calculating the square root of the average variance extracted (AVE) in each latent variable. The value of the square root should exceed the correlation shared between the constructs in the model (Fornell \& Lacker, 198I). The square root of the AVE is shown diagonally in Table 6.3, while the correlations between the latent variables represent the latent variable correlation. Table 6.2 shows that the results of the measurement model were acceptable, since all values exceed the standard levels. In either a reflective or a formative model, multicollinearity at the structural level is a potential risk. To test for the existence of multicollinearity, VIF coefficients are measured. In a well-fitting model these coefficients should not exceed the value of 5.0 (Hair et al., 20I I; Sarstedt et al., 20I4). In this model, the highest score is 3.73 , which is well below the threshold.

Table 6.3

The discriminant validity of the measurement model

Discriminant validity

Latent variable correlations

\begin{tabular}{lllllllllll} 
Constructs & SIB & KAB & INF & WP & SR & SN & $A B$ & ORG & GEN \\
\hline
\end{tabular}

Social

0,852

interaction

behaviour

(SIB)

Knowledge $\quad 0,614 \quad 0,812$

acquisition

behaviour

(KAB)

Informing $\quad 0,507 \quad 0,593 \quad 0,806$

(INF)

Work

$$
0,533 \quad 0,521 \quad 0,757 \quad 0,871
$$

Performance

(WP)

Social $\quad 0,45 \quad 0,353 \quad 0,559 \quad 0,74 I \quad I, 000$

reputation

(SR)

Subjective $\quad-0,168 \quad-0,227 \quad-0,19 \quad-0,226 \quad-0,118 \quad 0,894$

norm (SN)

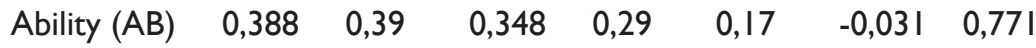

$\begin{array}{lllllllll}\text { Organisation } & 0,295 & 0,416 & 0,404 & 0,2 & 0,077 & -0,216 & 0,289 & 1,000\end{array}$

(ORG) 
Discriminant validity

Latent variable correlations

\begin{tabular}{llllllllll} 
Constructs & SIB & KAB & INF & WP & SR & SN & AB & ORG & GEN \\
\hline $\begin{array}{l}\text { Gender } \\
\text { (GEN) }\end{array}$ & 0,083 & 0,075 & 0,208 & 0,241 & 0,127 & $-0,157$ & $-0,061$ & 0,052 & 1,000 \\
\hline
\end{tabular}

\subsubsection{Structural model}

The measurement model showed satisfactory model quality, which clears the way to move to stage 2 of the PLS-SEM statistical analysis. This stage involves the assessment of the structural model. Unlike the co-variance-based SEM, PLS-SEM does not have a standard goodness-of-fit statistic, and thus far, no valuable corresponding statistics have been created (Henseler \& Sarstedt, 2013).A structural model was used to verify the hypotheses based on the path coefficients and R2 values (Chin \& Newsted, 1999). The R2 values were used to assess the ability of the model to explain the variance in the endogenous variables. The path coefficients indicate the statistical significance of the hypotheses. The significance levels of these path coefficients are estimated using the bootstrapping method, with a sub-sample of the minimally required 5000 (Hair et al., $20 \mathrm{II}$ ). The significance levels show whether the hypotheses have a significant influence in order to establish the theoretical model of the research.

In Table 6.4, the resulting values show the relationships between the exogenous and the endogenous variables in the structural model. The exogenous variables explain $43 \%$ of the variance in the endogenous variable knowledge acquisition behaviour, and the exogenous variables explain $36 \%$ of the variance in the endogenous variable social interaction behaviour. The results show a positive significant relationship for the endogenous variable social interaction behaviour and the exogenous variables work performance $(\mathrm{H} 2 \mathrm{~b} ; \beta=0.238, \mathrm{p}<0.05)$, social reputation $(\mathrm{H} 3 \mathrm{a} ; \mathrm{B}=0.164, \mathrm{p}<0.05)$, and ability $(\mathrm{H} 8 \mathrm{~b} ; \beta=0.2 \mathrm{I}, \mathrm{p}<0.00 \mathrm{I})$. For the other endogenous variable, knowledge acquisition behaviour, positive significant relationships are found with the variables informing $(\mathrm{HIa} ; \beta=0.302, \mathrm{p}<0.00 \mathrm{I})$, work performance $(\mathrm{H} 2 \mathrm{a} ; \beta=$ $0.198, p<0.05)$ and ability $(\mathrm{H} 8 \mathrm{a} ; \beta=0.166, \mathrm{p}<0.00 \mathrm{I})$. The subjective norms $(\mathrm{H} 4 \mathrm{a} ; \beta=0.088$, $\mathrm{p}$ $<0.05$ ) in the organisation are shown to be a negative, significant indicator for the knowledge acquiring behaviour, albeit with a minimal path coefficient. The exploratory character of this research, with its combination of different scales, leads to predominantly low path coefficients. Chin (1998) argues that a substantial relation can be considered from values larger than 0.2 , while Lohmöller (1989) even considers relations larger than 0.1 to be relevant. This implies that the path coefficients in this study represent a satisfactory value. The structural model has been controlled for the variables of gender and organisation. Organisation in this case is also significantly related to the social interaction and knowledge acquisition types of social behaviour. This implies that the manner in which an organisation organizes the CoP within the organisation is also an important indicator of the intensity of social behaviour within the CoP. 
Table 6.4

Summary of the results

\begin{tabular}{|c|c|c|c|c|c|}
\hline Hypothesis & $\begin{array}{l}\text { Exogenous } \\
\text { variable(s) }\end{array}$ & $\begin{array}{l}\text { Endogenous } \\
\text { variable(s) }\end{array}$ & $\begin{array}{l}\text { Path- } \\
\text { coefficients }\end{array}$ & $\begin{array}{l}\text { Adjusted } \\
\text { R2 }\end{array}$ & Result \\
\hline HIa & Informing & $\begin{array}{l}\text { Knowledge } \\
\text { acquisition } \\
\text { behaviour }\end{array}$ & $0.302 * *$ & 0.427 & Supported \\
\hline HIb & Informing & $\begin{array}{l}\text { Social } \\
\text { interaction } \\
\text { behaviour }\end{array}$ & 0.107 & 0.360 & $\begin{array}{l}\text { Not } \\
\text { supported }\end{array}$ \\
\hline $\mathrm{H} 2 \mathrm{a}$ & $\begin{array}{l}\text { Work } \\
\text { performance }\end{array}$ & $\begin{array}{l}\text { Knowledge } \\
\text { acquisition } \\
\text { behaviour }\end{array}$ & $0.198 * *$ & 0.427 & Supported \\
\hline $\mathrm{H} 2 \mathrm{~b}$ & $\begin{array}{l}\text { Work } \\
\text { performance }\end{array}$ & $\begin{array}{l}\text { Social } \\
\text { interaction } \\
\text { behaviour }\end{array}$ & $0.238 *$ & 0.360 & Supported \\
\hline H3 & $\begin{array}{l}\text { Social } \\
\text { reputation }\end{array}$ & $\begin{array}{l}\text { Social } \\
\text { interaction } \\
\text { behaviour }\end{array}$ & $0.164 *$ & 0.427 & Supported \\
\hline $\mathrm{H} 4 \mathrm{a}$ & $\begin{array}{l}\text { Subjective } \\
\text { norm }\end{array}$ & $\begin{array}{l}\text { Knowledge } \\
\text { acquisition } \\
\text { behaviour }\end{array}$ & $-0.088 *$ & 0.427 & Supported \\
\hline H4b & $\begin{array}{l}\text { Subjective } \\
\text { norm }\end{array}$ & $\begin{array}{l}\text { Social } \\
\text { interaction } \\
\text { behaviour }\end{array}$ & -0.045 & 0.360 & $\begin{array}{l}\text { Not } \\
\text { supported }\end{array}$ \\
\hline
\end{tabular}

The hypotheses $5 \mathrm{a}-\mathrm{b}$ to $7 \mathrm{a}-\mathrm{b}$ have been merged into hypotheses $8 \mathrm{a}$ and $8 \mathrm{~b}$ (see I 6.4.3)

\begin{tabular}{|c|c|c|c|c|c|}
\hline H8a & Ability & $\begin{array}{l}\text { Knowledge } \\
\text { acquisition } \\
\text { behaviour }\end{array}$ & $0.166 * *$ & 0.427 & Supported \\
\hline H8b & Ability & $\begin{array}{l}\text { Social } \\
\text { interaction } \\
\text { behaviour }\end{array}$ & $0.217 * * *$ & 0.360 & Supported \\
\hline $\begin{array}{l}\text { Control } \\
\text { variable }\end{array}$ & Organisation & $\begin{array}{l}\text { Knowledge } \\
\text { acquisition } \\
\text { behaviour }\end{array}$ & $0.189 * * *$ & 0.427 & Supported \\
\hline
\end{tabular}

$* \mathrm{p}<0.05 / * * \mathrm{p}<0.01 / * * * \mathrm{p}<0.001$ 


\begin{tabular}{llllll}
\hline Hypothesis & $\begin{array}{l}\text { Exogenous } \\
\text { variable(s) }\end{array}$ & $\begin{array}{l}\text { Endogenous } \\
\text { variable(s) }\end{array}$ & $\begin{array}{l}\text { Path- } \\
\text { coefficients }\end{array}$ & $\begin{array}{l}\text { Adjusted } \\
\text { R2 }\end{array}$ & Result \\
\hline $\begin{array}{l}\text { Control } \\
\text { variable }\end{array}$ & Organisation & $\begin{array}{l}\text { Social } \\
\text { interaction } \\
\text { behaviour }\end{array}$ & $0.121^{*}$ & 0.360 & Supported \\
\hline $\begin{array}{l}\text { Control } \\
\text { variable }\end{array}$ & Gender & $\begin{array}{l}\text { Knowledge } \\
\text { acquisition } \\
\text { behaviour }\end{array}$ & -0.048 & 0.427 & $\begin{array}{l}\text { Not } \\
\text { supported }\end{array}$ \\
\hline $\begin{array}{l}\text { Control } \\
\text { variable }\end{array}$ & Gender & $\begin{array}{l}\text { Social } \\
\text { interaction } \\
\text { behaviour }\end{array}$ & -0.017 & 0.360 & Not \\
\hline
\end{tabular}

\subsection{Discussion \& Conclusions}

In this chapter, we have presented and empirically tested a conceptual model aimed at identifying the perceived enablers that stimulate individuals to participate in a CoP. The sub-question formulated for this purpose is as follows:

What are the reasons, reports and perceptions of professionals about their knowledge acquisition and social-interaction behaviour that affect participating in Communities of Practice?

In answering this research question, we first focus on the latter part of the research question: social behaviour in CoPs. Due to the low levels of social behaviour in the examined CoPs, we have decided to reflect the results based on an ideal situation for community and organisational management. In many situations where a CoP is introduced, the ideal situation for a wellfunctioning CoP reflects the image outlined by Barab, MaKinster, \& Scheckler (2003) These authors describe the ideal situation as 'a persistent sustained social network of individuals, who share and develop an overlapping knowledge base, set of beliefs, values, history, and experiences focused on common practice and/or mutual enterprise (p.238). This model situation occurs only to a limited extent, especially in sponsored organisational CoPs initiated by the organisations; the situation described by Barab, MaKinster, \& Scheckler (2003) is the exception rather than the rule. In most situations, the dominant group consists of community members who mostly acquire knowledge (90\%). A much smaller percentage $(9 \%)$ contribute irregularly, and the smallest group ( $1 \%$ ) - the heavy contributors - are active users who account for most of the contributions in the CoP (Brandtzaeg \& Heim, 20I I).

In this study, the first components introduced in the MOAC framework were examined.This part of the study examines the components of the MOAC model on which the individual can exert influence. The motive to improve work performance, motive to improve social reputation, and the individual perceived ability measured as self-efficacy affect an individual's social interaction behaviour in a CoP. On the other hand, we have also tested whether there are relationships between the motivation to become informed, the motivation to improve work performance, or an individual's abilities (self-efficacy) and the individual's knowledge acquisition behaviour in a CoP. As an answer to the sub-question, we focus on the outcomes of the hypotheses. The results in this study show a high score for the individual's motivations and a relatively low score for the reporting of the individual's actual social behaviour in a CoP. This positive significant 
relationship between the motivations and social behaviour in the CoP requires a more nuanced interpretation. Increasing motivation has a direct effect on social behaviour, but the degree of social behaviour is low on an ideal typical scale - the scale indicating the ideal situation of organisation management and community managers. Consequently, the motivations should be stimulated or increased enormously to eventually lead to more participation by individuals. Being informed is an important motivation to acquire knowledge in a CoP $(\mathrm{HIa})$, which implies that a substantial group of community members visit the CoP from the motivation to gain the latest knowledge in the field but also to keep updated developments within the organisation. The uses and gratifications theory (Katz, Blumler, \& Gurevitch, 1973) underlines this by stating that the need to be informed is an important indicator of one's knowledge acquisition behaviour-a statement that is confirmed in the study of Lee \& Ma (20I2). The motive to improve work performance also corresponds with the knowledge acquisition behaviour $(\mathrm{H} 2 \mathrm{a})$ and the social interaction types of social behaviour $(\mathrm{H} 2 \mathrm{~b})$. Improving individual and collective performance in the workplace is an important motivation for community members who show more active types of social behaviour. This result is an important source of information for organisations to create a common goal. By cooperating with colleagues to formulate goals that determine the improvement of work performance and are also supported by the employees, the opportunity must be created to increase the collective willingness to participate more actively in CoPs. Plausible reasons for the emergence of this type of motivation can be found in, among others, recognizing the pool of expertise, experience and connection, which are also indicators of work performance (Seibert, 20I5). Improving one's social reputation is also positively related to the social interaction types of social behaviour in a CoP $(\mathrm{H} 3 \mathrm{a})$, and this finding corresponds to the position of Wasko \& Faraj (2005) that individuals perceive knowledge sharing as a way to build their reputation.

Bettiga, Lamberti, \& Noci (2018) studied the role of motivation and ability on a type of human behaviour (willingness to co-create) at a higher level, with a minimal number of indicators. These authors found significant evidence of the positive relationships between the constructs of motivation and ability and the willingness to co-create. In the current study, an individual's perceived ability was also positively related to social behaviour (both knowledge acquisition $(\mathrm{H} 8 \mathrm{a})$ and social interaction $(\mathrm{H} 8 \mathrm{~b}))$. This finding concurs with the results of Bettiga, Lamberti \& Noci (2018), since it appears that the community members who are more knowledgeable are more active participants than the community members who have lower levels of ability. This confirms that organisations need to increase their support mechanisms to increase the intensity of individuals' activity. Some of the options for community designers are to provide user-friendly toolkits, short training sessions or online support consultation hours or to connect novices with experts.

We found a significant but negative relationship between subjective norms and knowledge acquisition behaviour $(\mathrm{H} 4 \mathrm{a})$. This is somewhat contradictory with the literature, which states that subjective norms positively affect the intended and, ultimately, actual behaviour. However, in this study, subjective norms were operationalized as a form of social pressure that community members perceive. A negative relationship between subjective norms and social activity is therefore much more plausible. Since the values of the knowledge acquisition behaviour are low, this might indicate that participants register-under pressure from the organisation and colleagues - for a CoP, but when they see the low level of activity from others-except from the active community managers and ambassadors - they are less likely to make active contributions. The main objective of this study was to explore whether a more elaborated model can provide a better explanation of an individual's social behaviour in communal environments. An empirical 
study was conducted to test and validate the research model and to examine the specific impact of variables on different types of social behaviour in CoPs. Improving work performance and enhancing one's social reputation are two motivating factors for an individual's social interaction behaviour in a CoP.An individual's perceived ability to act in a CoP also stimulates his or her actual types of social interaction. Being informed, improving work performance and social pressure are the two significant indicators for starting knowledge-acquiring types of social behaviour, while one's perceived ability also contributes to the knowledge-acquiring types of social interaction.

This exploratory study is a first step towards testing individual social behaviour in a CoP by focusing on purely individual factors as reported in a survey. The focus will be on organisational factors in Chapter 7 and on the community factors that affect an individual's social behaviour in a CoP in Chapter 8. The model used in combination with the methodology contributes to our knowledge of individuals' changing social behaviour in a CoP. Using the perspective of the CMEM enables us to form a realistic image of factors that affect individual social behaviour and offers a starting point for interventions to stimulate this. The application of PLS-SEM is in this case the best option for testing the new MOAC model. Due to the exploratory nature of this research, PLS is used as the exploratory analysis method that functions as a prelude to an interpretive technique such as multiple linear regression or co-variance-based structural equation modelling. According to Henseler, Ringle \& Sinkovics (2009), PLS is a recommended method for statistical analysis in the early stages of theoretical development in order to test and validate exploratory models. This study is a first step towards the development of an explanatory model for the evaluation of individual and group behaviour in online CoPs. Future research should focus on improving the explanatory power of the variables in the model.

Coming to the limitations and directions for future research. As indicated, it was decided to use the extended MOAC framework as a basis for examining and explaining social behaviour in CoPs. The original MOA model approaches social behaviour from different angles and is therefore a rather extensive explanatory model compared with other social psychological models. However, the number of items used to construct a latent variable is minimal, especially for social reputation, which had one indicator. It is advisable to further explore the individual components of the research model. Therefore, future research requires a more extensive elaboration of the different latent variables to increase the explanatory power of the model. One of the other limitations is the construction of the exogenous variable ability, which is operationalized in this study as self-efficacy. This factor is based on perceptions and reports and forms a valid indicator for an individual's ability. However, it is not an actual measure of an individual's abilities. One can, for example, overestimate one's own capacities or give socially desirable answers. In line with this, we initially introduced nine indicators to measure three distinctive types of ability: perceived ability to express, perceived ability to contact and perceived digital skills. However, the results indicated that these three variables strongly correlate with each other, which instigated that we consider perceived ability as one latent variable with nine indicators. In line with the previous recommendation to improve the scale, we would highly recommend developing an improved scale for the 'ability' concept.

A third limitation in this study is related to the number of observers. Since most members in a CoP are observers, this group is in this study overrepresented. These members mainly consume knowledge (e.g., read posts, comments, newsletters) instead of creating or sharing knowledge. In addition, women respondents are over-represented, mainly due to the largely female-dominated care domain. For that reason, the results of the studies should be considered 
as a first empirical test of the MOAC framework, which needs to be validated and tested in more, larger and varied networks.

To conclude, our survey studied the relationships between the sub-constructs of the motivation: the motivation to be informed, the motivation to improve one's work performance, the motivation to improve one's social reputation and subjective norms with two types of social behaviour in a CoP, knowledge acquisition behaviour and social interaction behaviour. The findings indicate that when stimulating the two types of social behaviour in a CoP, it is worthwhile to focus on the motivation to improve work performance and to improve an individual's (perceived) ability to stimulate both types of social behaviour. Focusing on the motivation to be informed and subjective norms will mainly increase the knowledge acquisition behaviour and less strongly contribute to the often-preferred social behaviour. When the objective is to stimulate social interaction behaviour, a promising strategy seems to be focusing on the motivation to improve work performance, one's social reputation and one's (perceived) ability to perform. 



\section{7 \\ Organisational Factors Driving Social Behaviour in Communities of Practice}

\section{I. Introduction}

The research in this chapter examines organisational factors that affect an individual's social behaviour in a CoP. Organisations increasingly recognize the added value that CoPs can play in achieving their organisational goals. Knowledge sharing within these CoPs is recognized as one of the most fundamental organisational practices for improving organisational performance (Rasula, Vuksic, \& Stemberger, 2012). Therefore, organisations increasingly start initiatives that support the development of these practices. Some examples are e-learning sessions, expert consultations, conferences, and informal and CoPs. In this study, the focus is on the last: The CoP.The CoP enables an organisation to unite employees in order to improve, for example, mutual trust, cooperation, innovative capacity, work efficiency, and work effectiveness (Lee et al., 20I5). However, the initiation, design and organisation of a CoP by an organisation's management board is often a complicated project. There is a great deal of debate regarding what organisation management can and must do to stimulate the development of CoPs in the organisation because "people are the key actors in online communities, and they cannot be shaped or programmed in the way physical materials or software can" (Kraut \& Resnick, 20II, Pp. 6). The ambition of organisations to successfully design a CoP from scratch is therefore often an illusion. Nonetheless, organisations can always steer the development to some extent. Organisations can play an important role in fulfilling the preconditions that stimulate social interactions of community members. CoPs specifically organized for the benefit of both the employee and the organisation require not only participation and effort from the individual but also effort and support from the organisation.

As shown in Chapter 6, the motivation to be informed, to improve work performance and self-efficacy are important indicators for an individual's social behaviour in a CoP. However, not only the individual factors determine individual social behaviour, organisational factors also play a prominent role in this. Opportunities form external conditions that stimulate an individual's social activity in CoPs (e.g. Siemsen et al., 2008). For example, bottom-up projects initiated by employees should be stimulated and supported by the organisation (i.e., through time and social and technological support). However, if an individual professional wants to share his or her knowledge in a CoP with experts with a common interest and/or expertise, these professionals also need time to perform the knowledge exchange and facilities in which to do so (Baran \& Cagiltay, 20l0). Organisations that offer their professionals opportunities to collaborate may structurally affect an individual's active behaviour within information systems. An opportunity represents the extent to which the situation is conductive to completing a particular action (Maclnnis \& Jaworski, 1989), and it includes the contextual factors that facilitate or impede certain behaviours (Gruen et al., 2007; Macinnis et al., 1991). Hughes (2007) argues that opportunity is just as the ability of an individual-a moderating variable that influences the link between motivation and behaviour. Nonetheless, opportunity can also be a factor that directly affects an individual's social behaviour if that person is participating because the conditions offered by the organisation are up to par. Individual activities within virtual collective 
environments may be negatively affected by a lack of external resources, such as time offered by an organisation (Baran \& Cagiltay, 20 I0; Szulanski, 1995). The autonomy for individuals to act in the manner they prefer (Cardona, Lawrence, \& Bentler, 2003; Schneckenberg, 2009) and the facilitating conditions that make it possible for individuals to participate (Bhattacherjee, 200 I; Venkatesh et al., 2003) are two stimulating factors. The opportunity of an individual to act in a certain manner is measured in various ways. Hughes (2007) conducted an extensive study to evaluate the application of the MOA model in different contexts and manners. Siemsen et al. (2008) has found significant evidence for the relationship between time availability-one of the variables in the factor of opportunity-and knowledge sharing. If an organisation offers its professionals time to participate in a CoP, these professionals are more likely to participate. However, the opposite is also possible. When individuals perceive a great deal of time pressure during their working day, it is very likely that they will be less prone to actively participate in knowledge sharing activities with colleagues.

Determining which factors affect social behaviour within groups, CoPs and other social activities remain an important area of research. In Chapter 6, the first part of the MOAC model was examined - the individual perspective. In this chapter, the second part of the MOAC model will be explored-the organisational perspective. We expect that an individual's action is largely affected by his or her motivation and ability to act, the opportunities offered to the individual, and the perception of communality within the online environment. The level of support that the community members receive from the organisation is included under the construct of 'opportunity', which is an organisational factor.A few hypotheses are presented concerning the influence of opportunity on an individual's social behaviour within online communities. These hypotheses are tested using the same sample as that in Chapter 6. In the remainder of this chapter, the underlying concepts of the construct opportunity are briefly described, the method of the research is briefly reviewed, and the results are introduced and discussed in the context of the following sub-question:Which organisational factors do professionals report as potential drivers of and barriers to their social behaviour in CoPs?

\subsection{Theory}

The subdivision between knowledge acquisition behaviour and social interaction behaviour used as the primary types of social behaviour in Chapter 6 are also used in Chapters 7 and 8. The potential organisational factors will be introduced and explained in the paragraphs below.

\subsection{Opportunity}

Many studies have been conducted to test the influence of the work environment on job performance (Fuller, Marler, \& Hester, 2006; Rajagopal et al., 2012; Theurer, Tumasjan, \& Welpe, 2018). Regarding the context of this research project, the focus is on the relationship between the work environment - the organisational context-and the social activities of community members in a CoP.The definition used for a CoP, which was introduced in Chapter 2, is as follows: "a social network where people in an organisational context come together around a common topic, passion or interest and regularly interact on- and offline with a focus on knowledge management, innovation, learning and social networking". The social activities in a CoP can be split into two concepts: knowledge acquisition behaviour and social interaction behaviour. The first refers to the knowledge-consuming behaviour of individuals in a CoP (Lampe et al., 20I0), and the latter refers to the more active, contributing behaviour of an individual in a CoP (Faraj, von Krogh, Monteiro, \& Lakhani, 20l6). 
The literature has shown that there is a strong relationship between the suitability of the work environment for collaborating and distributing knowledge and the actual social behaviour in these kinds of activities (Fuller et al., 2006; Kang, Lee, \& Kim, 2017; Pyrko et al., 2017). Organisational management retains an important role in influencing the behaviour of employees. In this chapter, the focus is on three of the preconditions that can be facilitated or stimulated by organisational management: the time to participate that is offered by the organisation, the individual work autonomy that is provided, and the facilitating conditions which are optimized by the organisation. In the following paragraphs, the concepts are briefly discussed.

\section{Time}

The growth in the adoption of information and communication technology (ICT) has resulted in a transition from purely physical CoPs to increasingly virtual CoPs. This mitigates the constraints of time and space (Correia et al., 2009) and implies that community members who are willing to actively contribute to a CoP have more options to participate. They can access the online platform supporting the CoP anytime and anywhere. However, employees are often short on time, squeezing in their efforts in the CoP between tasks as they go about their day. In many cases, this results in limited time investment from the employees in the CoP (Pyrko et al., 2017). Despite the fact that many researchers mention a lack of time as an important aspect in determining actual behaviour (Hara \& Hew, 2007; Ndlela \& Du Toit, 200I), most academics did not take this aspect into account when studying the social behaviour of practitioners in CoPs. For example, Hew and Hara (2007) mention that social behaviour in CoPs is commonly hindered by a lack of time. Wu, Zhu, Zhong and Wang (20I2) also declare that time and effort are crucial aspects that could impact social behaviour in CoPs. Leaders in an organisation must be aware of the value of investing in knowledge management, but at the same time, knowledge management also requires the investment of allowing people to take time for knowledge sharing, knowledge capturing, consulting, and networking, for example (Ndlela \& Du Toit, 200 I). Ölander and Thøgersen (1995) also emphasize the importance of the factor of time and define it as an important aspect of social behaviour; in their motivation, opportunity, and ability framework, they define time as a situational factor for opportunity. Time represents the extent to which the situation is conductive to completing a particular action (Maclnnis \& Jaworski, 1989), and it includes the contextual factors that facilitate or impede certain behaviours (Macinnes et al., 1991; Gruen et al., 2006). The results of this discussion around the factor of time are considered in the following hypotheses:

HIa: A lack of time negatively relates to the level of knowledge acquisition behaviour in a community of practice.

H I b: A lack of time negatively relates to the level of social interaction behaviour in a community of practice.

\section{Work-autonomy}

Professionals are often perfectly able to make informed decisions that contribute to the realization of valuable outcomes for organisations, the group and the individual. The individual's perception of a job's motivating potential of a CoP for a professional is an important indicator in this (Cardona, Lawrence, \& Bentler, 2003). If professionals are given the freedom to organize their own independent work processes (e.g., plan their own working days and working habits), a situation arises in which most professionals return optimal results (Theurer et al., 2018). Particularly in asynchronous environments, such as CoPs where the virtual component plays a pivotal role, professionals must be able to make decisions in organizing their activities themselves. The increased workload and accompanying expected flexibility that the employee must have 
also require a similar attitude from the employer.This shift in autonomy at work is often defined as a shift towards workplace autonomy or job autonomy (Slemp, Kern, \& Vella-Brodrick, 20I5). Professionals in organisations increasingly combine on-site and off-site working, which increases the professionals' work autonomy (Van Yperen,Wörtler, \& De Jonge, 2016).According to these authors, "blended working refers to smooth and seamless time- and location- independent working, which increases workers' job autonomy and intrinsic work motivation" (Van Yperen, Wörtler, \& De Jonge, 2016 , p. I8I.). However, it is also plausible that it is the other way aroundthat an individual's work autonomy contributes to the intensity of blended working, which might occur in several forms (e.g., blended learning, CoP participation, network events).

Scheduling specific time to actively participate in knowledge management processes is one activity-stimulating activity, giving individuals the autonomy to decide when and how to carry out specific tasks in the organisation (Foss, Minbaeva, Pedersen, Reinholt, 2009) is another. When employees are given this freedom, they have control over setting their own schedules and procedures used to do perform in a meaningful manner. Many studies have found a relationship between work autonomy and social activity, personal initiative, and the feeling of responsibility (Fuller, Marler, \& Hester, 2006; Parker, Wall, Jackson, 1997). Gagnè (2003) found evidence that work autonomy strongly relates to engagement in prosocial behaviour. Especially with the increasing job demands in today's workplace, the desire of employees to manage their own agendas is increasing. An increase in work autonomy can, according to Van Yperen et al. (2016), maintain employee work motivation in the case of perceived job demands being high. This implies that when ICT is effectively used-by blending on-site and off-site workingsubstantial positive consequences can be realized for employees' effectiveness and the quality of their work. In addition, such effective use might have a positive influence on an individual's responsibility for task performance (Fuller et al., 2006). An employee who feels that he or she has work autonomy can decide how to effectively use a knowledge management system and when to share knowledge through it (Kang et al., 2017).

As discussed, autonomy is strongly connected to the freedom to determine one's own schedule, especially in knowledge-intensive organisations. However, the freedom of expression is also an important form of autonomy (Schepers \& Van den Berg, 2007). Having a culture in which one is allowed to have a different opinion is a typical example of this. Shih \& Huang (20l4) argue that talking freely and openly to others is an indication of a shared trust relationship-a situation that is needed to increase individual social behaviour in CoPs. The combination of the freedom of speech and the autonomy to determine one's own schedule helped in formulating $\mathrm{H} 2 \mathrm{a}$ and $\mathrm{H} 2 \mathrm{~b}$.

H2a: Work autonomy positively relates to the level of knowledge-acquisition behaviour in a community of practice.

H2b: Work autonomy positively relates to the level of social interaction behaviour in a community of practice.

\section{Facilitating conditions}

In the adoption of (new) technologies, organisations often focus on creating an atmosphere by ensuring optimal facilitating conditions. The concept of facilitating conditions was introduced in the technology acceptance model (TAM) (Venkatesh, Morris, Davis \& Davis, 2003) and can be defined as organisational support for users of technology that can influence system use (Peñarroja, Sánchez, Gamero, Orengo, \& Zornoza, 2019). Venkatesh (2003) points to the organisational and technical infrastructure available in an organisation to support the use of 
information technologies as a typical example of facilitating conditions. Venkatesh's work shows that the organisation has a fundamental role in supporting employees in collaboration and knowledge-exchange processes. The conditions created by organisations are the services offered by an organisation to encourage users to adopt a certain technology. The conditions generally also refer to individuals' perceptions of the accessibility of technological and/or organisational resources (i.e., knowledge, resources and training) available to help them use a system for the benefit of their own work (Venkatesh, Morris, Davis \& Davis, 2003). These conditions form a fundamental indicator for the adoption of a technology because when users have insufficient training or significant difficulties for using the system, the intention to use the technology is reduced. Research on Jeon, Kim, \& Koh (20II) and Peñarroja et al. (2019) has shown that optimal facilitating conditions contribute to community members' social behaviour in physical and virtual CoPs. Bock, Kankanhalli, \& Sharma (2006) also found a significant relationship between, on the one hand, the facilitating conditions and, on the other hand, the use of electronic knowledge repositories to maximize an individual's learning performance. Thus, optimal facilitating conditions have a positive influence on the use of the offered technologies (Nistor et al., 20l4). One of the likely consequences of optimal facilitating conditions in an organisation is posed by Peñarroja et al. (2019). These authors state that organisational facilitating conditions give the impression to the community members that the organisation values social behaviour in the CoP, which ultimately may encourage members to collaborate more frequently. However, facilitating conditions are also often seen as a necessary precondition for social behaviour in general. Without optimal support for the use of organisational technologies, the chances of social software being adopted dramatically decrease.

The role of facilitating conditions in the context of social behaviour in CoPs has resulted in the following two hypotheses.

H3a: Facilitating conditions (technological and methodological support) positively relate to the level of knowledge acquisition behaviour in a community of practice.

H3b: Facilitating conditions (technological and methodological support) positively relate to the level of social interaction behaviour in a community of practice.

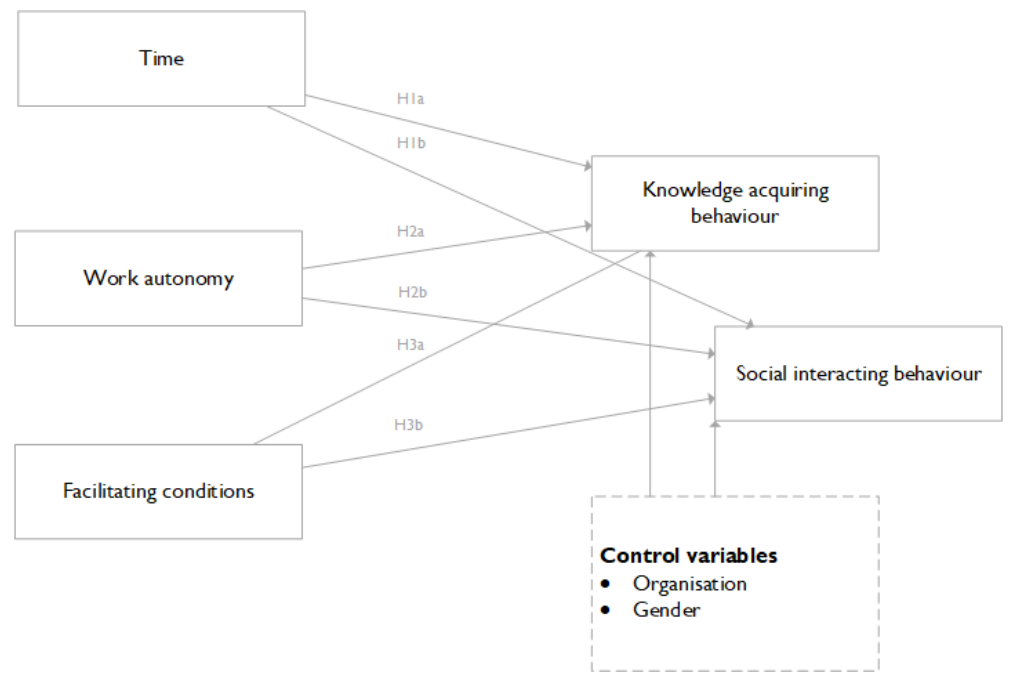

Figure 7.I. Conceptual Model Organisational Perspective 


\subsection{Method of research}

In Chapters 6,7 and 8, the same method of research is used to test the relationship between the MOAC variables and the level of social behaviour in CoPs. Therefore, in all these chapters, the number of respondents is 221 professionals from four different cases, with the same sample characteristics as defined in Chapter 6 (Table 6.I). The types of social behaviour have been measured in the same manner with the same 6-point scale that measures the intensity of social behaviour ranging from never to multiple times a day as in Chapter 6. The three exogenous variables are also measured with the same 7-points Likert scale to measure time (2 items), work autonomy ( 3 items) and facilitating conditions ( 2 items). The scales used in this survey can be found in Appendix 7.I.

An informal selection interview with the managers responsible for the development of the CoP was used to determine the extent to which the social platform meets the characteristics of a CoP.The four cases differ in nature, but all the CoPs use the same online community software, with the same options for social interaction. The method defined in Chapter 6 is also used in this study to ensure the replicability of the research.

\subsection{Data Analysis and results}

The partial least squares (PLS) techniques of structural equation modelling (SEM), which uses a principal component-based estimation, were used for the analysis. Unique to partial least squares structural equation modelling (PLS-SEM), when compared to other statistical modelling techniques, is the fact that PLS techniques enable researchers to model, estimate, and simultaneously test complex theories with empirical data while accounting for measurement error. PLS-SEM focuses on the analysis of variance and is particularly useful for this research due to its capability to validate predictive models with a limited number of respondents and with a skewed distribution. The specific tool that was used for this analysis was SmartPLS 3.0, which was created by Ringle,Wende, \&Will (2005). It can test two sub-models in a structural equation model: the structural model, which specifies the relationships between the independent and dependent latent variables, and the measurement model, which specifies the relationships between the latent variables and their observed indicators. The properties of PLS-SEM make it suitable for exploratory research settings that are "simultaneously data-rich and theoryprimitive" (Sarstedt et al., 20l4). The method to test for common method variance described in Chapter 6 has also been used in this chapter. The common method variance score for this test is $33,4 \%$, which is well below the threshold of $50 \%$. This shows that the common method variance is not of great concern for this study and it is therefore unlikely that it biases the interpretation of our results.

Table 7.I

Constructs, variables (codes), sources, number of items, composite reliability, mean, standard deviation, and average variance extracted (AVE).

\begin{tabular}{lccc} 
Construct Variable Source $\quad$ Items & $\begin{array}{l}\text { Composite Mean \& SD AVE } \\
\text { reliability }\end{array}$ \\
\hline
\end{tabular}

\begin{tabular}{llllll}
$\begin{array}{l}\text { Social } \\
\text { behaviour }\end{array}$ & $\begin{array}{l}\text { Social } \\
\text { interaction } \\
\text { behaviour }\end{array}$ & $\begin{array}{l}\text { Millen, Fontaine, } \\
\text { \& Miller (2002) }\end{array}$ & 0.905 & $2.13(0.96)$ & 0.66 \\
\hline
\end{tabular}




\begin{tabular}{|c|c|c|c|c|c|c|}
\hline Construct & Variable & Source & Items & $\begin{array}{l}\text { Composite } \\
\text { reliability }\end{array}$ & Mean \& SD & AVE \\
\hline & $\begin{array}{l}\text { Knowledge } \\
\text { acquisition } \\
\text { behaviour }\end{array}$ & $\begin{array}{l}\text { Bock, Orces, } \\
\text { Kim, \& Lee } \\
(2005)\end{array}$ & 2 & 0.788 & $3.13(1.11)$ & 0.66 \\
\hline \multirow[t]{3}{*}{ Opportunity } & Time (TI) & $\begin{array}{l}\text { Orlikowski } \\
\text { (1993) }\end{array}$ & 2 & 0.973 & $3.50(1.73)$ & 0.95 \\
\hline & $\begin{array}{l}\text { Work } \\
\text { autonomy } \\
\text { (WA) }\end{array}$ & $\begin{array}{l}\text { Cardona, } \\
\text { Lawrence \& } \\
\text { Bentler (2003); } \\
\text { Salanova, Peiró } \\
\text { \& Schaufeli } \\
(2002)\end{array}$ & 3 & 0.910 & $5.50(1.13)$ & 0.77 \\
\hline & $\begin{array}{l}\text { Facilitating } \\
\text { conditions } \\
\text { (FC) }\end{array}$ & $\begin{array}{l}\text { Bhattacherjee, } \\
\text { (200I); } \\
\text { Venkatesh et al., } \\
(2003)\end{array}$ & 2 & 0.981 & $4.13(1.81)$ & 0.96 \\
\hline
\end{tabular}

\subsection{Measurement model}

The approach used in this chapter is largely identical to the one used in Chapter 6. Even as in Chapter 6 with the individual factors, in this chapter the organisational factors in this conceptual model are also reflective in nature, and each latent variable is reflected in a few indicators and tested as such.

Reliability and validity are calculated in the measurement model by means of indicator reliability (Appendix 7.2) internal consistency, convergent validity, discriminant validity, and testing for multicollinearity. Given de exploratory nature of this study, the indicator reliability is of an acceptable level with scores between .63 and .98 (Hulland, 1999). The composite reliability for each of the model's constructs is above the recommended level of 0.7 , indicating good internal consistency of the data (Hair et al., 20 I I). Regarding the convergent validity, the scores are all well above the recommended 0.5 advised by Bagozzi \& Yi (1988). This indicates that all latent variables, on average, explain more than half of the variance in its indicators. A confident discriminant validity is realized when the squared root of the average variance extracted (AVE) is above 0.50 and that for each construct, it is higher than the correlation between it and the other variables. Table 7.2 shows that the square root of the AVE (diagonal values) of each latent variable is greater than its correlations with the other latent variable. The discriminant validity is thus reached, and based on that, we can conclude that the main constructs measure different aspects. Garson (2016) indicates that the Fornell-Lacker criterion-the squared AVE-is an accepted method for assessing the discriminant validity of a measurement model.

The risk of multicollinearity has been determined by testing for the indicator's variance inflation factor (VIF) value. This value must-according to Sarstedt et al. (20I4) —be kept as low as possible but must at least be below a threshold of 5 . The values in this model are well below this value, with the highest score being I,547. 
Table 7.2

The discriminant validity of the measurement model

Discriminant validity

Latent variable correlations

\begin{tabular}{llllllll}
\hline Constructs & SIB & KAB & TI & WA & FC & ORG & GEN \\
\hline $\begin{array}{l}\text { Social interaction } \\
\text { behaviour (SIB) }\end{array}$ & 0,808 & & & & & \\
$\begin{array}{l}\text { Knowledge } \\
\text { acquisition } \\
\text { behaviour (KAB) }\end{array}$ & 0.625 & 0,791 & & & & & \\
Time (TI) & 0.098 & 0.164 & 0,974 & & & \\
$\begin{array}{l}\text { Work autonomy } \\
\text { (WA) }\end{array}$ & 0.284 & 0.338 & 0.147 & 0,878 & & \\
$\begin{array}{l}\text { Facilitating } \\
\text { Conditions (FC) }\end{array}$ & 0,429 & 0,463 & 0.231 & 0.483 & 0,981 & \\
$\begin{array}{l}\text { Organisation } \\
\text { (ORG) }\end{array}$ & 0.293 & 0.440 & 0.134 & 0.210 & 0.335 & 1,000 & \\
Gender (GEN) & 0.068 & 0.051 & 0.024 & -0.099 & -0.062 & 0.052 & I,000 \\
\hline
\end{tabular}

\subsubsection{Structural model}

Given the adequate measurement model in this study, the hypotheses can be tested by examining the structural (inner) model. We applied the PLS-SEM procedure based on SmartPLS 3.0 and analysed the coefficient of determination (R2), which is the significance of the structural path coefficients. The structural model was constructed to test the relationship between the exogenous variables and the endogenous variables. As discussed in Chapter 6, the PLS-SEM does not have an adequate goodness-of-fit measurement-a fact that must be considered when interpreting the results. In the bootstrapping, the recommended 5000 subsamples (Lee et al., 2015) were performed to test the statistical significance of each PLS path coefficient using t-tests. The estimates for the PLS path coefficients are used to examine the direct effects of the hypothesized relations between the endogenous and exogenous variables.

The endogenous constructs in this model received rather small R2 values - smaller than the rule of thumb outlined by Hair et al. (20II). These authors consider R2 values of $0.75,0.50$, and 0.25 for endogenous latent variables to be substantial, moderate and weak, respectively. However, this determination fully depends on the field of research, as, according to Garson (20I6), a value of 0.25 might be considered "high" if the state of the art in the given subject and field had previously led to values that were even lower. Especially in cases of the unpredictable humans, low R2-values often occur. In this research, the level of activity and the commitment 
from the community members largely differ, which has implications for the explained variance. The explained variance in this model is minimal for both endogenous variables, $22.3 \%$ for the social interaction behaviour and $32.4 \%$ for the knowledge acquisition behaviour.

Table 7.3 indicates a positive significant relationship between the facilitating conditions in an organisation and the actual intensity of social behaviour by the community members, segregated by knowledge acquisition behaviour $(\mathrm{H3a} ; \beta=0.292, \mathrm{p}<0.00 \mathrm{I})$ and social interaction $(\mathrm{H} 3 \mathrm{~b} ; \beta=$ $0.340, p<0.00 \mathrm{I})$. Work autonomy is significantly related to the knowledge acquisition behaviour $(\mathrm{H} 2 \mathrm{a} ; \beta=0.138, \mathrm{p}<0.05)$. Work autonomy is not significantly related to the social interaction behaviour, while time has no significant relationship with both types of social behaviour.A path coefficient larger than 0.2 is considered substantial, argues Chin (1998), and Lohmöller (1989) even considers relations larger than 0.1 to be relevant.

In this chapter with a focus on the organisational factors, more attention is given to the differences between the organisations. Regarding the control variables, both organisation and gender have positive significant effects on the dependent variables-namely, male (mean = 2.03 , s.d. $=0.1 \mathrm{I})$ and female $($ mean $=2.17$, s.d. $=.08)$ versus the social interaction behaviour and male $($ mean $=2.93$, s.d. $=0.13)$ and female $($ mean $=3.21$, s.d. $=.09)$ versus the knowledge acquisition behaviour. Regarding the organisations, the financial service organisation (mean = 2.15 , s.d. $=0.16$ ), large hospital $($ mean $=1.78$, s.d. $=0.09)$, youth care institution (mean = 2.60 , s.d. $=0.13)$, and organisation for the care of disabled people $($ mean $=2.28$, s.d. $=0.12)$ controlled for the social interaction behaviour. The financial service organisation (mean $=3 . \mathrm{II}$, s.d. $=0.16)$, large hospital $($ mean $=2.62$, s.d. $=0.1 \mathrm{I})$, youth care institution $($ mean $=3.80$, s.d. $=0.15$ ), and organisation for the care of disabled people (mean $=3.39$, s.d. $=0.13$ ) controlled for the knowledge acquisition behaviour. Due to the skewed distribution of the sample with a disparate distribution for both the participants from the organisation and gender, controlling for these variables is desirable. Omitting these control variables from the model results in a reduced model variance. Skewed distributions in CoPs is not illogical since the activity levels differ greatly per individual.

In the questionnaire, the respondents also provide their function in the organisation. Segmenting these functions into two groups leads to the following categories: managers and practitioner. It is especially interesting whether there are differences between managers and employees regarding their reporting of the time they have, to participate in the CoP.As a result, especially the managers significantly report more time to show social interaction behaviour $(B=0.212)$, while the employees often lack time to show this type of behaviour $(B=-0.70, p<0.05)$ ). 
Table 7.3

Summary of the PLS results

\begin{tabular}{|c|c|c|c|c|c|}
\hline Hypothesis & $\begin{array}{l}\text { Exogenous } \\
\text { variable(s) }\end{array}$ & $\begin{array}{l}\text { Endogenous } \\
\text { variable(s) }\end{array}$ & $\begin{array}{l}\text { Path- } \\
\text { coefficients }\end{array}$ & $\begin{array}{l}\text { Adjusted } \\
\text { R2 }\end{array}$ & Result \\
\hline Hla & Time & $\begin{array}{l}\text { Knowledge } \\
\text { acquisition } \\
\text { behaviour }\end{array}$ & 0.037 & 0.324 & $\begin{array}{l}\text { Not } \\
\text { supported }\end{array}$ \\
\hline HIb & Time & $\begin{array}{l}\text { Social } \\
\text { interaction } \\
\text { behaviour }\end{array}$ & -0.016 & 0.223 & $\begin{array}{l}\text { Not } \\
\text { supported }\end{array}$ \\
\hline $\mathrm{H} 2 \mathrm{a}$ & $\begin{array}{l}\text { Work } \\
\text { Autonomy }\end{array}$ & $\begin{array}{l}\text { Knowledge } \\
\text { acquisition } \\
\text { behaviour }\end{array}$ & $0.138 *$ & 0.324 & Supported \\
\hline $\mathrm{H} 2 \mathrm{~b}$ & $\begin{array}{l}\text { Work } \\
\text { Autonomy }\end{array}$ & $\begin{array}{l}\text { Social } \\
\text { interaction } \\
\text { behaviour }\end{array}$ & 0.105 & 0.223 & $\begin{array}{l}\text { Not } \\
\text { supported }\end{array}$ \\
\hline $\mathrm{H} 3 \mathbf{a}$ & $\begin{array}{l}\text { Facilitating } \\
\text { Conditions }\end{array}$ & $\begin{array}{l}\text { Knowledge } \\
\text { acquisition } \\
\text { behaviour }\end{array}$ & $0.292 * * *$ & 0.324 & Supported \\
\hline H3b & $\begin{array}{l}\text { Facilitating } \\
\text { Conditions }\end{array}$ & $\begin{array}{l}\text { Social } \\
\text { interaction } \\
\text { behaviour }\end{array}$ & $0.340 * * *$ & 0.223 & Supported \\
\hline $\begin{array}{l}\text { Control } \\
\text { variable }\end{array}$ & Organisation & $\begin{array}{l}\text { Knowledge } \\
\text { acquisition } \\
\text { behaviour }\end{array}$ & $0.304 * * *$ & 0.324 & Supported \\
\hline $\begin{array}{l}\text { Control } \\
\text { variable }\end{array}$ & Organisation & $\begin{array}{l}\text { Social } \\
\text { interaction } \\
\text { behaviour }\end{array}$ & $0.156 * *$ & 0.223 & Supported \\
\hline $\begin{array}{l}\text { Control } \\
\text { variable }\end{array}$ & Gender & $\begin{array}{l}\text { Knowledge } \\
\text { acquisition } \\
\text { behaviour }\end{array}$ & 0.065 & 0.324 & $\begin{array}{l}\text { Not } \\
\text { supported }\end{array}$ \\
\hline $\begin{array}{l}\text { Control } \\
\text { variable }\end{array}$ & Gender & $\begin{array}{l}\text { Social } \\
\text { interaction } \\
\text { behaviour }\end{array}$ & $0.091 *$ & 0.223 & Supported \\
\hline
\end{tabular}




\subsection{Discussion \& Conclusions}

A CoP is usually initiated by the management of an organisation that sets certain goals from a strategic point of view. Introducing a CoP can then contribute to achieving these goals. In this chapter, the primary focus is on those factors that lie within the sphere of influence of the organisation. Interventions by organisations regarding these factors should contribute positively to the individual social behaviour of colleagues in CoPs. This identification of the organisational factors is captured in the following sub-question:

Which organisational factors do professionals report as potential drivers of and barriers towards their social behaviour in CoPs?

The results are a clear reflection of most CoPs worldwide; most people are active on a minimal level. However, even though an appropriate number of observers are tolerable for online CoPs, too many observers might have negative consequences for the viability of the CoP (Sun et al., 2014). Stimulating active behaviour in a CoP is therefore fundamental for the survivability of the CoP.This is also shown in the relationships in the model. The role that an organisation can play in increasing the chances of the sustainable development of CoPs within the organisation has been tested in this study with three concepts: time, work autonomy, and facilitating conditions. As an answer to the sub-question, the first result shows a positive significant relationship between work autonomy and the level of knowledge acquisition behaviour $(\mathrm{H} 2 \mathrm{a})$ in the CoP and not with the social interaction type of behaviour. This implies that individuals have the freedom to contribute but that their social behaviour is limited to the consumption types of social behaviour in a CoP.A possible explanation for this result may be that participants copy the behaviour of others. The group of passive users who only read content is overrepresented in the CoPs. When (new) users always see the same people contributing, the other participants might see the CoP as a club. Here, then, is a role for the community manager or, at a higher level, for organisational management to stimulate an individual to interact. In addition, community management must emphasize that making relevant contributions contributes to meaningful knowledge exchange within the organisation and thereby a positive improvement of practice. By setting the right example that reflects the desired behaviour, management shows their commitment to prove to the other community members that participating in the CoP is a valuable activity when improving their work practices (Martinsons, Davison, \& Huang, 2017; $\mathrm{Xu}$, Quaddus, \& Gao, 20I4). An extensive literature review was conducted by Sun et al. (20I4) to identify the primary factors that explain the behaviour of observers. One of the primary barriers for observers to act in the way they act is the fact that pro-social behaviour is not stimulated. Liao \& Chou (20l I) argue that observers are more affected by the shared vision of the group; therefore, enhancing pro-sharing norms would have a stronger influence on observers than on posters.

The positive significant relationships between the facilitating conditions and the level of social behaviour (both knowledge acquisition $(\mathrm{H} 3 \mathrm{a})$ and social interaction $(\mathrm{H} 3 \mathrm{~b})$ ) imply that the organisation has adequate support for using collaborative technologies. A result that corresponds with the findings of Jeon, Kim, \& Koh (20II), stating that facilitating conditions significantly affects knowledge-sharing behaviour. However, in this study, the actual intensity of social behaviour largely fluctuates for the social interaction types of social behaviour $\left(x^{-}=2.13\right.$, s.d. $=0.96)$ and the more passive types of social behaviour $\left(x^{-}=3.13\right.$, s.d. $\left.=1 . I I\right)$. This indicates that enough attention is paid to the digital component of the CoP but that the participants are not immediately convinced to make an active contribution themselves. A plausible explanation 
for this phenomenon is given by Fadel \& Durcikova (20l4) and Peñarroja et al. (2019), who state that organisational constructs, such as facilitating conditions or other organisational conditions, can be viewed as enabling environmental mechanisms to support knowledge exchange. Thus, providing optimal support in the use of the supporting technologies for the CoP is an important condition for individuals to act in a CoP.

Time allocated by the organisation is not recognized as a fundamental factor that affects an individual's social behaviour in a CoP.A plausible explanation for this can be found in the work of Pyrko et al. (2017). These authors state that the more inactive community members argue that a lack of time is the primary reason for their inactivity. In that case, the factor of time is used as an argument to explain their behaviour. However, a more extensive review of their interview data resulted in another explanation for a limited activity: the lack of clear immediate value of the CoP does not justify their time investment. If community members recognize the (immediate) value of the CoP, their willingness to participate will often increase simultaneously. Some of these triggers are engaging discussions, easily applicable lessons, relevant documents and valuable contacts. Thus, if one recognizes the added value of a CoP, one's willingness to invest time in or make time for social behaviour in a CoP increases. The literature review conducted by Nistor et al. (20I5) suggests that it is not only an individual's willingness to invest time in a CoP that is affected when the individual perceives the immediate value of the CoP. The amount of time invested by a community member positively relates to that member's sense of community. However, Nistor et al.'s study shows evidence to the contrary. Time spent in the CoP may apparently decrease the sense of community.A possible explanation they give is that community newcomers may be more enthusiastic about the new social environment, and this enthusiasm may fade in time. However, an additional study towards differences between managers and employees regarding their reported time and the types of social behaviour they conduct showed significant differences. Managers report more time to socially interact in a CoP compared to the employees who predominantly indicate insufficient time to participate. This confirms the contradictory between the perceptions of the manager and the employee. The manager feels that there is enough time to share and develop knowledge, while the employees do not share this feeling (Bun,Vries, Kolfschoten, \& Veen, 20I I).

Another significant positive relationship exists between the organisation and the levels of social behaviour. The levels of activity largely differ by organisation, which follows the organisational differences depicted in Chapter 4 and is also an unsurprising result. The more active CoPs in this sample exist in a situation in which the CoP is the primary source of information and the employees work in different locations. In particular, situations where employees work in different locations are stimulating for collaborative activities, a result confirmed by research of Karam, Straus, Byers, Kase, \& Cefalu (2018). These authors state that in their study, teachers who had co-located peers tended to participate more often in online collaborative activities than did those with more local colleagues.

The development of a quantitative scale with a set of organisational factors makes it possible to have a more thorough understanding of the opportunities for organisations to stimulate the development of CoPs. The inclusion of organisational factors as an indicator of social behaviour therefore also appears to be an important condition for meaningful interpretations of community developments, one of the objectives of the CMEM.The study symbolizes a new step towards better monitoring and control of community development in organisations. The final scale, with 7 items to measure the organisational factors and 7 items to measure the knowledge acquisition behaviour and social interaction behaviour, provides practitioners with potential 
points for improvement in current practices and developments in their CoPs. Researchers should interpret these variables as a first step in which organisational factors that contribute to better insight in the monitoring and evaluation of developments in CoPs are identified. For future research, it is recommended that the scale for the three organisational factors included in this research be elaborated upon. The fact that this research is part of a larger study on the influence of individual, group and organisational factors on individual social behaviour in CoPs made us decide to reduce the length of the questionnaire in order to ensure that the response rate was satisfactory and reliable ${ }^{2}$. 



\section{8 \\ Community Factors Driving Social Behaviour in Communities of Practice}

\section{I. Introduction}

Chapter 7 presents the specific organisational factors that contribute to the social behaviour of individuals in a CoP.These results provide insight into those factors that can be controlled and/ or facilitated by the management of an organisation. In this chapter, the final component of the MOAC model will be tested; this section primarily focuses on the community-specific factors that might contribute to individuals' social behaviours in the CoPs.

Building on the idea of connectivism, organisations are increasingly trying to create an atmosphere in which individuals, departments and groups can work together. Organisations are increasingly trying to support employees in order to improve cooperation, increase their competitive position, improve their innovative capacity and be able to operate more efficiently and effectively in a continuously changing market. One of the answers of organisations to these developments is the initiation of CoPs. Especially for the sake of knowledge management, collaboration and social bonding in a CoP, it is an important concept. By initiating a CoP, organisations often aim to reduce the social distance between the layers in an organisation and to guarantee high-quality knowledge management. A fundamental precondition for calling a CoP a CoP is the perception of social bonding in the workplace (Ifinedo, 20l4) in the form of attachment, commitment and involvement with other members and with the organisation, as well as mutual personal norms (Hirschi, 2002). Hooff \& Ridder (2004) emphasize the relevance of having employees who are committed to the organisation as an important indicator for knowledge-donating activities.

Introduction an online community software package is one step; designing a relevant and sustainable CoP is the next-often more complicated-step. Whether a CoP is perceived as a CoP is a matter of perception and experience. Some people experience a certain platform as a CoP, while others define it as a knowledge management system. In this chapter, the factor of communality is introduced as an exploratory concept to define the extent to which community members recognize typical characteristics of a communal environment. It is assumed that the extent to which community members feel committed to a CoP contributes to the type and intensity of their social behaviour in the CoP. In the context of this study, communality is operationalized in three typical characteristics of a CoP. These characteristics are a common identity, a mutual language and the degree of shared leadership. Therefore, the factor of communality is divided into three variables: shared identity, shared language and shared leadership. The more intense one perceives the concepts to be, the larger one's sense of community. In this chapter, we examine the possible relations between the three factors under the concept of communality in relation to the degree of social behaviour in a CoP.

Taking the abovementioned factors into consideration results in the following sub-question:

Which community factors affect the social behaviour of professionals in a community of practice? 


\subsection{Theory}

In the former chapters, the first three components of the original MOA framework were analysed from two perspectives: the individual perspective and the organisational perspective. In Chapter 6, a distinction is made between the different types of social interaction in a CoP. The definitions and operationalization described in that chapter also apply in this chapter. In this section, the last explanatory factor introduced in the MOAC model will be examined: the communality. Communality represents the group perspective regarding individual community behaviour. In this section, we introduce the concepts underlying the communality already briefly introduced in Chapter 3.

\subsection{Communality}

The unique mind-set that exists within a CoP is one of "communality". Communality holds a few facets of social capital, namely, social identification, shared vision and shared language, complemented by shared leadership. One of the unique features of CoPs is the lack of a single leader who controls the activities and outcomes in the groups. Zhu, Kraut, \& Kittur (20I2) posit that leadership behaviours within online communities should come from members at all levels, not simply from people in high-level leadership positions. Another characteristic of a CoP is shared language (Cadiz, Sawyer, \& Griffith, 2009). Members in a CoP create a common vocabulary to interact with each other to facilitate the transfer of information. Having a shared language may also be a way in which groups establish a sense of exclusivity (Cadiz et al., 2009). However, shared language may also increase the interactions between individuals within communities, which reduces the barrier to participate. This component of a CoP also strongly aligns with the aspect of a specific component of the description posed by Wenger, McDermott, \& Snyder (2002). One of the components in their structural model is domain. A domain is the shared identity that must be established in a CoP in order for the CoP to become effective. According to Chiu, Huang, Cheng, \& Sun (2015, p. 505), domain is the "community identification of an individual's sense of oneness with or emotional bond to the online community". In the following paragraphs, we will pay special attention to shared identity, shared language, and shared leadership as important factors which are expected to affect individual social behaviour in a CoP.

\section{Shared identity}

A domain is the sense of common identity that a CoP must establish to be effective (Cadiz et al., 2009, p. I04I). Shared identity, group identity and collective identity are three terms that are often used interchangeably. Such identity is often seen as one of the fundamental determinants for an individual's obligation to a community and its members (Figueiredo \& Scaraboto, 20 I6). Crowley, Mcadam, Cunningham, \& Hilliard (2018) even name identity as one of the fundamental components of a CoP.According to Marshall (1997, p.185), "identity is the principle that is most fundamental to all self-organizing systems. It contains the organisation's meaning, purpose, and intentionality and provides the coherence around which system stability emerges".

Specifically, in the context of CoPs, Wenger, Trayner \& De Laat (201 I, p.9) confirm Marshall's (1997) statement by claiming that a community becomes a community when it meets the precondition that it stimulates "the development of a shared identity around a topic or set of challenges". One definition for a shared identity is introduced by Turner, Hogg, Oakes, Reicher \& Wetherell (1987, p.843), who state that a shared identity "in everyday life emerges when people define a collection of people as members of the same category". However, limiting 
shared identity to only occurring when one is recognized as a member of a CoP is probably too limited for a convincing definition of shared identity. The question remains: what makes an identity a shared identity? To a large extent, a part of the answer lies in an individual's social identity and the group norms and values existing in the CoP. Ellemers, Gilder, Haslam, \& Gilder (20l4) introduce social identification, which is composed of social identity and group norms, as an important determinant of the shared identity in a CoP. According to these authors, it is important for community management to create a sense of shared identity to energize, direct and sustain work-related behaviours. The group norms then act as guidelines for an individual's own behaviour. Acting according to the shared identity depends on the willingness of the individual to identify with groups that seem to contribute to a positive sense of self, such as high-status or high-power groups (Haslam, Postmes, \& Ellemers, 2003). A limited sense of shared identity in a CoP reduces the chance of members having trust in each other and the possibility that mutual trust in general will develop (Lundkvist, 2004).

Providing support in a CoP to create a shared identity is therefore one of the tasks of community management. By using group identity theory as a perspective to look at the development of a shared identity, it becomes clear that CoP leadership, especially in the initial phases of a CoP, contributes to the development of a shared identity (Borzillo et al., 20I I). Ultimately, joint activities, mutual involvement, and openness and transparency contribute to creating a climate in which the development of a shared identity can arise. Ren et al. (20I2) found evidence that community members with a strengthened perception of group identity are more frequent visitors to the community and that their self-reported self-attachment grows proportionally. Nahapiet \& Ghoshal (1998) argued that identification acts as a resource influencing the motivation to combine and exchange knowledge. However, the opposite also occurs, distinct and contradictory social identities within groups constitute significant barriers to the creation, sharing and acquisition of knowledge.

This downside is also acknowledged by Wenger, Trayner \& De Laat (20I I), who state that a shared identity might also become an obstacle for outsiders to participate in the community. In that case, the shared identity forms a barrier for potentially relevant participants, a critical consequence for increasing social distances. Wenger, Trayner \& De Laat (20I I) emphasize that it is fundamental to stimulate network-building activities to reduce the barriers (Wenger,Trayner \& De Laat, 20I I).

However, it is expected that the perception of a shared identity in the CoP positively contributes to an individual's social behaviour. This leads to the following two hypotheses:

H la: Shared identity positively relates to the level of knowledge acquisition behaviour in a community of practice.

H I b:Shared identity positively relates to the level of social interaction behaviour in a community of practice.

\section{Shared language}

CoPs are completely dedicated to the social interactions of individuals within the community. For these social interactions to take place in a meaningful way, it is fundamental that the participants speak the same language: a shared language. Speaking about a shared language is not only about the language itself; according to Lesser and Storck (200I, p.836), it is also about "the acronyms, subtleties, and underlying assumptions that are the staples of day-to-day interactions". It facilitates common ground for the community members so that messages and 
signals are understood by everyone within that community. The creation of an own vocabulary with terms and jargon relevant in the context of a certain task also prevents others from interpreting the information. Liao \& Chou (20II) found significant evidence that a shared language positively affects the attitude towards a member's knowledge adoption. It makes the exchange of knowledge more efficient and effective.Wenger (1998) suggests an interdependent process of social behaviour and reification of knowledge. Behaviour in a CoP revolves, to a large extent, around the concept of 'reification', which means "giving concrete form to something that is abstract and which mainly comes from manifesting the experience of the participants. Reification in CoPs can take the form of tools, procedures, stories or language" (Hildreth \& Kimble, 2004, p. 27). To become understood by colleagues and peers in a certain domain and to stimulate the process of reification, it is important to create a common language that is understood by the relevant people. It allows people to communicate with each other, regardless of their work location and the existence of mutual relationships. If participants in a CoP do not speak the 'same language', problems arise in the transfer of knowledge, cooperation and mutual communication. Chiu, Hsu, \&Wang (2006) found evidence that a shared language affects the information quality in a community and is therefore seen as a vital precondition for social behaviour in CoPs.A shared understanding among CoP members is supported when building an own vocabulary, enhancing the efficiency of communication in the CoP (Chang \& Chuang, 20 I I). Speaking the same language is expected to positively contribute to an individual's social experience. This leads to the following two hypotheses:

H2a: Shared language positively relates to the level of knowledge acquisition behaviour in a community of practice.

H2b: Shared language positively relates to the level of social interaction behaviour in a community of practice.

\section{Shared leadership}

Equality in social groups is an important incentive for participants to increase their activity, as it contributes to the feeling of mutual trust (Edelmann et al., 2017). Thus far, the focus has been purely on team structure to encourage members to engage in the practice of shared leadership. The focus should be more on the desired behaviour. A study by Wood (2005) suggests that members engaging in shared leadership practices have to feel that they are also legitimized to interchangeably take the role of a community leader within the community's organizing structure. By giving more responsibilities to the community members, these members become able to express and experience a greater sense of commitment and empowerment within the communities.

Approaching the concept of shared leadership only from the perspective of organisational structure is, as already discussed by Wood (2005), often insufficient. This rather new form of leadership should be seen as a set of actions rather than a designated role (Johnson et al., 20 I5), it is therefore very close to a degree of equality. Pearce \& Sims (2000, p. I I6) introduced shared leadership as "the process of shared influence between and among individuals". Communities often do not have a formal leader who is responsible for the direction of the community, but leadership often emerges through interactions. Johnson et al. (20I5, p. I67) point out that "the combination of open, voluntary participation and the paucity of formal leadership roles in online communities means that leadership is inherently shared". Although everyone can enact some level of leadership behaviour in a CoP, this does not imply an equal distribution and the same effectiveness of leadership behaviours across individuals (Zhu et al., 20I2). According to Johnson et al. (20I5), a shared form of leadership is strongly represented in online communities 
focused on knowledge exchange. Therefore, the perception of equality in CoPs might be a motivating factor for an individual's social behaviour in a CoP. Although the body of literature related to the shared nature of online community leadership is not very large, the shared leadership phenomenon is, according to Johnson et al. (20I5) and Levina \& Arriaga (20I4), in line with findings about developing roles, less stringent boundaries, and the individual's position taken to obtain or maintain influence in online communities.

The final hypothesis in this chapter focuses on the role of shared leadership in the social behaviour of community members in a CoP; the result is the following hypotheses:

H3a: Shared leadership positively relates to the level of knowledge acquisition behaviour in a community of practice.

H3b: Shared leadership positively relates to the level of social interaction behaviour in a community of practice.

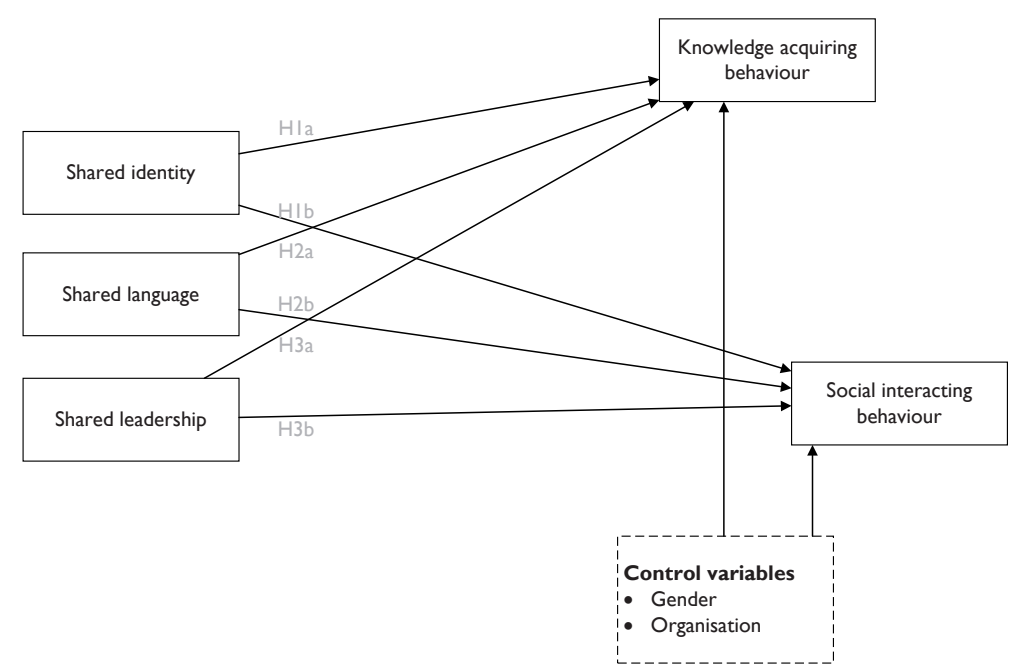

Figure 8.I. Conceptual Model - Community Perspective

\subsection{Method of research}

In the survey described in Chapter 6, data have been collected to analyse those factors that affect the social behaviour of individuals in CoPs. In this chapter, a subset from the questionnaire especially related to the concept 'communality' is used with the underlying variables: shared leadership, shared language and shared identity. These three constructs were measured with a 7 -point Likert scale varying from I $=$ fully disagree to $7=$ fully agree, and the score level of activity was measured with a scale for intensity varying from I = never to 6 = multiple times a day. The scales used in this survey can be found in Appendix 8. I. These factors are related to the factor social behaviour, which was tested in the previous chapters. The sample distribution in this research is the same as the one in Chapter 6 and thus has an unequally divided group in terms of gender and organisation (Table 6.I).

As discussed in Chapter 6, this model has also been evaluated using partial least squares structural equation modelling (PLS-SEM) and in this chapter the same steps have been taken as in the previous two chapters. First, the common method variance (CMV) has been tested by 
means of the Harman's single-factor test. The result is a satisfying score of $33.95 \%$, which is well below the threshold of $50 \%$ (Podsakoff et al., 2003). Therefore, it is assumed that CMV is not of great concern and thus will bias the interpretations of the findings.

\subsection{Results}

In Table 8. I, relevant background information is given to explain the design of the scale, but also to indicate what the underlying descriptive statistics are.

Table 8.I

General statistics of the group scales

\begin{tabular}{|c|c|c|c|c|c|c|}
\hline Construct & Variable & Source & Items & $\begin{array}{l}\text { Composite } \\
\text { reliability }\end{array}$ & $\begin{array}{l}\text { Mean \& } \\
\text { SD }\end{array}$ & AVE \\
\hline \multirow[t]{2}{*}{$\begin{array}{l}\text { Social } \\
\text { behaviour }\end{array}$} & $\begin{array}{l}\text { Social } \\
\text { interaction } \\
\text { behaviour }\end{array}$ & $\begin{array}{l}\text { Millen, } \\
\text { Fontaine, \& } \\
\text { Miller (2002) }\end{array}$ & 5 & 0.905 & $2.13(0.96)$ & 0.66 \\
\hline & $\begin{array}{l}\text { Knowledge } \\
\text { acquisition } \\
\text { behaviour }\end{array}$ & $\begin{array}{l}\text { Bock, Orces, } \\
\text { Kim, \& Lee } \\
(2005)\end{array}$ & 2 & 0.788 & $3.13(1.11)$ & 0.65 \\
\hline \multirow[t]{3}{*}{$\begin{array}{l}\text { Commu- } \\
\text { nality }\end{array}$} & $\begin{array}{l}\text { Shared } \\
\text { Language } \\
\text { (SLa) }\end{array}$ & $\begin{array}{l}\text { Chiu et al } \\
(006)\end{array}$ & 3 & 0.933 & $5.03(1.01)$ & 0.82 \\
\hline & $\begin{array}{l}\text { Shared } \\
\text { Leadership } \\
\text { (SLe) }\end{array}$ & $\begin{array}{l}\text { Woods } \\
(2005)\end{array}$ & 5 & 0.967 & $5.50(1.13)$ & 0.94 \\
\hline & $\begin{array}{l}\text { Shared } \\
\text { Identity } \\
\text { (SI) }\end{array}$ & $\begin{array}{l}\text { Woods } \\
(2005)\end{array}$ & 2 & 0.893 & 4.91 (1.04) & 0.81 \\
\hline
\end{tabular}

\subsection{Measurement model}

The first step in the PLS-SEM analysis is creating the measurement model. In this model, we examine the reliability and validity of the constructs in the model through indicator reliability (Appendix 8.2) convergent validity, internal consistency, reliability of measures, discriminant validity, and testing for multicollinearity. The indicator reliability does not show concerning (cross-)loadings, with loadings between .62 and .97. Following the guidelines from Hulland (1999) and Kwong-Kay Wong (2013), who indicate that in case of exploratory research loadings of .40 are acceptable. The scores for this scale are all well-above this threshold.

Regarding convergent validity, the model shows - as set out in Table 8.I - that each latent variable's average variance extracted (AVE) is above the acceptable threshold of 0.5 (Bagozzi $\& \mathrm{Yi}$, 1988). This implies that the items used to measure the constructs are indeed related. To measure the internal consistency reliability of the measures, the composite reliability measure is used as an indicator. Composite reliability scores above 0.7 indicate a satisfying internal 
consistency reliability (Hair et al., 20I I). In this model, the lowest score is 0.799 , well above the requested 0.700 . For measuring the discriminant validity, the Fornell-Larcker Criterion analysis for checking discriminant validity is used. In the diagonal of the table, the square root of the average variance extracted (AVE) is presented, and the other numbers represent the latent variable correlation. If the square root of the AVE is larger than the latent variable correlations, the discriminant validity is established (Fornell \& Larcker, 198I). Table 8.2 shows the discriminant validity in the measurement model. The discriminant validity is measured by calculating the square root of the average variance extracted (AVE) in each latent variable. The value of the square root should exceed the correlation shared between the constructs in the model (Fornell \& Lacker, I98I). The square root of the AVE is shown diagonally in Table 8.2, while the correlations between the latent variables represent the latent variable correlation. Table 8.I shows that the results of the measurement model were acceptable, since all values exceed the standard levels.

The VIF values are calculated to test the model for multicollinearity. The results show that all values score well below the threshold of 5 (Sarstedt et al., 20I4), with the highest score being 3.856. This indicates that the structural model results are not negatively affected by collinearity.

Table 8.2

The discriminant validity of the measurement model

Discriminant validity

Latent variable correlations

\begin{tabular}{|c|c|c|c|c|c|c|c|}
\hline Constructs & SIB & $\mathrm{KAB}$ & SI & SLa & SLe & ORG & GEN \\
\hline $\begin{array}{l}\text { Social interaction } \\
\text { behaviour (SIB) }\end{array}$ & 0,807 & & & & & & \\
\hline $\begin{array}{l}\text { Knowledge } \\
\text { acquisition } \\
\text { behaviour (KAB) }\end{array}$ & 0.625 & 0,790 & & & & & \\
\hline Shared identity (SI) & 0,443 & 0,404 & 0,898 & & & & \\
\hline $\begin{array}{l}\text { Shared language } \\
\text { (SLa) }\end{array}$ & 0,418 & 0,395 & 0,677 & 0,907 & & & \\
\hline $\begin{array}{l}\text { Shared leadership } \\
\text { (SLe) }\end{array}$ & 0,423 & 0,391 & 0,666 & 0,676 & 0,968 & & \\
\hline $\begin{array}{l}\text { Organisation } \\
\text { (ORG) }\end{array}$ & 0,294 & $0,44 I$ & 0,274 & 0.281 & 0,251 & 1,000 & \\
\hline Gender (GEN) & 0,069 & 0,049 & 0,052 & 0,120 & $0,1 \mid I$ & 0.052 & 1,000 \\
\hline
\end{tabular}

\subsubsection{Structural model}


The measurement model has shown acceptable results, which ensures that the structural model can be tested. The structural model in this study presents the statistics for the path significance of hypothesized relationships between the endogenous and exogenous variables by using path coefficients $(B)$ and $R$-squares $\left(R^{2}\right)$. The strength of the relationship between the variables is indicated by $B$, which represents the impact of each exogenous variable on the correlations. The PLS-SEM results for the $B$ and $R^{2}$ values are shown in Table 8.3. The significance levels of the path coefficients are estimated using the bootstrapping method, with a sub-sample of 5000 . The significance levels show whether the hypotheses have a significant influence in order to establish the theoretical model of the research.

A summary of the results with the significant relationships can be found in Table 8.3. The endogenous constructs knowledge acquisition behaviour and social interaction behaviour show rather small R2 values (respectively $31 \%$ and $26 \%$ ), which is not necessarily a problem since the purpose of the model is to explain unpredictable and highly variable individual social behaviour (Garson, 2016).The results illustrate that the shared identity perceived by community members affects their levels of social behaviour; significant positive relationships show this for knowledge acquisition behaviour $(\mathrm{HIa} \beta=0.148, \mathrm{p}<0.05)$ and social interaction behaviour $(\mathrm{HI} ; \beta=0.209$, $P<0.0 \mathrm{I})$. Another significant positive relationship exists between the individual's perception of shared leadership in the CoP and the social interaction type of social behaviour in the CoP $(\mathrm{H} 3 \mathrm{~b} ; \mathrm{B}=0.159, \mathrm{p}<0.0 \mathrm{I})$ and not between the shared leadership and knowledge acquisition behaviour $(\mathrm{H} 3 \mathrm{a}) \mathrm{A}$ relationship between shared language and the two types of social behaviour is minimal and not significant, which leads to not accepting $\mathrm{H} 2 \mathrm{a}$ and $\mathrm{H} 2 \mathrm{~b}$.

Despite the relatively low scores on the path coefficients, these remain significant. Chin (1998) considers path coefficients higher than 0.2 to be valid indicators, while Lohmöller (1989) even considers relations larger than 0.1 to be substantial. The results are controlled for the variables organisation and gender. Organisation is significantly related to both types of social behaviour, which implies that there are relatively large differences between the reported intensity of the social interaction between the organisations. Just as in the Chapters 6 and 7, the results must thus be interpreted with caution.

Table 8.3

Summary of the results

\begin{tabular}{llllll}
\hline Hypothesis & $\begin{array}{l}\text { Exogenous } \\
\text { variable(s) }\end{array}$ & $\begin{array}{l}\text { Endogenous } \\
\text { variable(s) }\end{array}$ & $\begin{array}{l}\text { Path- } \\
\text { coefficients }\end{array}$ & $\begin{array}{l}\text { Adjusted } \\
\text { R2 }\end{array}$ & Result \\
\hline Hla & $\begin{array}{l}\text { Shared } \\
\text { identity }\end{array}$ & $\begin{array}{l}\text { Knowledge } \\
\text { acquisition } \\
\text { behaviour }\end{array}$ & $0.148^{*}$ & 0.307 & Supported \\
\end{tabular}




\begin{tabular}{|c|c|c|c|c|c|}
\hline Hypothesis & $\begin{array}{l}\text { Exogenous } \\
\text { variable(s) }\end{array}$ & $\begin{array}{l}\text { Endogenous } \\
\text { variable(s) }\end{array}$ & $\begin{array}{l}\text { Path- } \\
\text { coefficients }\end{array}$ & $\begin{array}{l}\text { Adjusted } \\
\text { R2 }\end{array}$ & Result \\
\hline HIb & $\begin{array}{l}\text { Shared } \\
\text { identity }\end{array}$ & $\begin{array}{l}\text { Social } \\
\text { interaction } \\
\text { behaviour }\end{array}$ & $0.209 * *$ & 0.259 & Supported \\
\hline $\mathrm{H} 2 \mathrm{a}$ & $\begin{array}{l}\text { Shared } \\
\text { language }\end{array}$ & $\begin{array}{l}\text { Knowledge } \\
\text { acquisition } \\
\text { behaviour }\end{array}$ & 0.106 & 0.307 & $\begin{array}{l}\text { Not } \\
\text { supported }\end{array}$ \\
\hline $\mathrm{H} 2 \mathrm{~b}$ & $\begin{array}{l}\text { Shared } \\
\text { language }\end{array}$ & $\begin{array}{l}\text { Social } \\
\text { interaction } \\
\text { behaviour }\end{array}$ & 0.125 & 0.259 & $\begin{array}{l}\text { Not } \\
\text { supported }\end{array}$ \\
\hline H3a & $\begin{array}{l}\text { Shared } \\
\text { leadership }\end{array}$ & $\begin{array}{l}\text { Knowledge } \\
\text { acquisition } \\
\text { behaviour }\end{array}$ & 0.144 & 0.307 & $\begin{array}{l}\text { Not } \\
\text { supported }\end{array}$ \\
\hline H3b & $\begin{array}{l}\text { Shared } \\
\text { leadership }\end{array}$ & $\begin{array}{l}\text { Social } \\
\text { interaction } \\
\text { behaviour }\end{array}$ & $0.159 * *$ & 0.259 & Supported \\
\hline $\begin{array}{l}\text { Control } \\
\text { variable }\end{array}$ & Organisation & $\begin{array}{l}\text { Knowledge } \\
\text { acquisition } \\
\text { behaviour }\end{array}$ & $0.335^{* * * *}$ & 0.307 & Supported \\
\hline $\begin{array}{l}\text { Control } \\
\text { variable }\end{array}$ & Organisation & $\begin{array}{l}\text { Social } \\
\text { interaction } \\
\text { behaviour }\end{array}$ & $0.162 * *$ & 0.259 & Supported \\
\hline $\begin{array}{l}\text { Control } \\
\text { variable }\end{array}$ & Gender & $\begin{array}{l}\text { Knowledge } \\
\text { acquisition } \\
\text { behaviour }\end{array}$ & -0.005 & 0.307 & $\begin{array}{l}\text { Not } \\
\text { supported }\end{array}$ \\
\hline $\begin{array}{l}\text { Control } \\
\text { variable }\end{array}$ & Gender & $\begin{array}{l}\text { Social } \\
\text { interaction } \\
\text { behaviour }\end{array}$ & 0.018 & 0.259 & $\begin{array}{l}\text { Not } \\
\text { supported }\end{array}$ \\
\hline
\end{tabular}

\subsection{Discussion \& Conclusions}

The previous two chapters (6 \& 7) focused on the individual and organisational factors that affect an individual's social behaviour in a CoP. In this chapter, the relationship between an individual's perception of the presence of community-specific characteristics in a CoP to which they belong and the individual types of social behaviour they perform are examined. Even though research on social behaviour in CoPs has been conducted from various perspectives, 
the actual characteristics of a CoP are often left out of consideration. In this study, three characteristics of a CoP are tested as indicators of social behaviour in a CoP..The sub-question to be answered is as follows:

\section{Which community factors affect the social behaviour of professionals in a community of practice?}

Before answering this question, we first focus on the endogenous variables in this studynamely, the degree of social behaviour, split into two types of social behaviour: the knowledge acquisition behaviour and the social interaction behaviour. In general, the rather low R2 values and path coefficients in the conceptual model for the community perspective are largely caused by the large variance in the social behaviour of individuals in a CoP.A CoP that is introduced within an organisation often attracts a great deal of attention from a number of 'ambassadors', such as the communication department or HR, who are familiar with various forms of communication and knowledge exchange.A substantial proportion of the other colleagues-or (potential) participants-recognize the relevance of the CoP but see insufficient importance in actively participating (Harris, 20I5). This also explains to a considerable extent why, in general, the $R^{2}$ values are low, especially those that focus on common behaviour. Thus, the results of this study provide an indication of how to improve our understanding of communality and increase the level of the communality.

In the study, three independent variables (shared identity, shared language, and shared leadership) were examined with the two dependent variables (knowledge acquisition behaviour and social interaction behaviour). The results of this study show that from a community-based perspective, the strongest enabling factor is the individual's perception of a shared identity (e.g., belonging to a group and shared norms and values) versus the two types of social behaviour in a CoP: knowledge acquisition behaviour $(\mathrm{HIa})$ and social interaction behaviour $(\mathrm{HIb})$. According to the social identity theory, people base their social identity on belonging to a certain group (Shih \& Huang, 20I4), which is strongly related to the perceived internalization operationalized in the shared norms and values. A study of Garas, Garcia, Skowron, \& Schweitzer (20I2) has shown that social norms play an important role in shaping the behaviour of users in online communities. Therefore, it is recommended that pro-sharing norms-norms that stimulate members to distribute their knowledge to others-be stimulated in order to motivate members to be of value for the community-a statement that is acknowledged by Orlikowski (1993). The fact that a shared identity stimulates an individual's social behaviour in a CoP also confirms the statement of Kimmerle et al. (2013) that with a growing collective identity, the knowledge repertoire and practice-related resources increase. When a shared identity-consisting of, for example, mutual interests and goals-increases, the level of social activity in a CoP grows proportionally. This also corresponds with the findings of Bonacich \& Schneider (1992), cited in Sun et al. (20I4, p.II2), who state that "communities with a stronger group identity usually have a greater number of member contributions". Thus, it is recommended that practitioners focus on the development of a shared identity when designing and/or developing a CoP within an organisational context.

The second positive significant relationship exists between the perception of a form of shared leadership with the social interaction behaviour in a $\operatorname{CoP}(\mathrm{H} 3 b)$.A positive relationship has also been found between shared leadership and knowledge acquisition behaviour, albeit less strongly and not significantly.A plausible explanation for the latter is that community members primarily acquiring knowledge and information from the CoP, are less inclined with the 'culture' in the CoP and thus have - in general - a less strong feeling about the degree of (shared) leadership. The positive significant relationship between shared leadership and social interaction behaviour in a 
CoP is in line with the results of the study of Day, Gronn, \& Salas (2004) and the argumentation of Johnson et al. (20I5). The latter state that leadership in online communities emerges through social interactions. They argue that "the combination of open, voluntary participation and the paucity of formal leadership roles in online communities means that leadership is inherently shared" (Johnson et al., 2015, p. 167). Many online communities, including those studied in this dissertation, give formal authority to designated administrators and moderators, thereby giving them the freedom to perform the role in their own way. However, these formal authorities are often recruited from the most active and engaged participants and are needed for an online community to grow from a couple of users to a large group. Nevertheless, as the results indicate, these people are not recognized as formal, hierarchical leaders. Therefore, this seems to indicate that these formal authorities are promoting freedom of expression and communication without imposing too much formal regulation. This ultimately leads to participants having the feeling that they are making decisions together without the hassle of a hierarchical order.

The absence of a positive significant relationship between shared language and intensity in performing the types of social behaviour is remarkable. No significant evidence has been found that having a common language contributes to the intensity of social behaviour, which confirms the findings of Chiu et al. (2006), who state that having a shared language contributes only to the quality of the social interactions and not by definition the quantity. However, it was our assumption that having a shared language-which implicitly indicates an equivalent level of thinking and a form of commonality - contributes to an increased intensity of social behaviour. To conclude, the aim of this chapter was to explore the role of perceived shared identity, perceived shared language, and perceived shared leadership in individual social behaviour in a CoP.An empirical study was conducted to test and validate the research model and to investigate the impacts of the variables. The empirical results show that perceived shared identity has a significant positive relationship with the intensity of knowledge acquisition behaviour and social interaction behaviour, while shared leadership is only positively related to the intensity of social interaction behaviour. In addition, no significant evidence was found for a possible relationship between the shared leadership and knowledge acquisition behaviour and for having a shared language and the different types of social behaviour. Focusing on the development of a shared identity and a shared form of leadership without hierarchy or individual leaders is likely to influence the intensity of individual social behaviour of community members in a CoP. Providing special attention to activities and tactics to improve these two shared concepts is therefore recommended in order to improve both types of social behaviour in a CoP-setting ${ }^{3}$.

\footnotetext{
${ }^{3}$ Since parts of the survey introduced in Chapter 6 predominantly apply to Chapters $7 \& 8$, the general limitations also apply to these chapters.
} 



\section{PART IV: GROUP BEHAVIOUR IN COMMUNITIES OF PRACTICE}

In the Chapters 6, 7 and 8, we used a traditional theoretical model in which we searched for causal relationships between explanatory variables on the one hand and perceived types of social behaviour on the other.Actual behaviour will be central in the coming chapters. The fact that social relationships are increasingly being collected and stored within the online platforms - by means of web-based social interaction data - offers us the opportunity to analyse actual behaviour. Part IV revolves around answering the following research question: How does group behaviour develop in a community of practice?

In Chapter 9, the web-based social interaction data will be used to analyse community development patterns. These patterns reflect the group processes in communities of practice over a certain period.

Chapter 10 deals with the creation of network positions within communities of practice. In addition, this chapter will link the survey answers from Chapter 6 till 8 of those community members who have given permission for this to the web-based social interaction data. In doing so, we test whether the MOAC variables are related to these network positions.

In the final chapter, the social interaction patterns of the community members will be analysed in depth by using ERGMmodels. Besides that, the constructs from the survey were linked as attributes to the community members to test whether relationships exist between the social interaction pattern and the attributes of the individual community members.

Parts of this dissertation will be used for future scientific publications 



\section{9 \\ Development Phases of Communities of Practice}

\subsection{Introduction}

In Part III, developments in a CoP were examined from the individual's perspective by testing the relationship between individual, organisational and community factors and an individual's social behaviour in a CoP. In this chapter, the developments in CoPs are approached from another perspective, namely, from the point of view of community development patterns. In general, professionals can use online community software to interact, consult with peers and create, improve or reference knowledge, regardless of time or place (Cook-Craig \& Sabah, 2009). However, at present, there is limited understanding of the functioning of CoPs and what leads to an increase or decrease in the health of these communities. The digital traces collected and stored in the online community software offer new, more 'data driven' opportunities to improve our insights into these developments. It is the question of how we can describe and analyse behaviour patterns in CoPs based on these digital traces.

To answer these questions, three steps will be followed: (I) identify and formalize the developments in the CoPs through the identification of community development patterns, (2) identify community member roles in CoPs, and (3) analyse relationships between community developments and the distribution of member types.This description of community development patterns, member types and the relationships between them enables us to describe CoP developments, understand them, and recommend interventions. These three steps come together in one sub-question:Which aspects of community development patterns characterize the development of a community of practice? Fulfilling this objective supports us in revealing the relationship between the structure of CoPs and the actual behaviour of individuals in a dynamic atmosphere.

\subsection{Theory}

\subsection{Continuing Professional Development}

Organisations and their employees have a mutually interdependent relationship with each other (Holt Larsen, 2004). An organisation expects motivated, professional, and highly informed employees who are continuously willing to improve themselves and their practices, while employees want and must continuously develop themselves to meet their own ambitions as well as those of their organisation. Therefore, the professional development of individuals within an organisation is of high priority for most organisations. Where once the focus was on formal learning in training programmes, organisations increasingly come into a stage of creating a professional culture of lifelong and continuous learning with a focus on social learning in communal spaces. The role of social learning in communal spaces has gained increasing attention due to its capacity to foster the professional development of individuals and improve business outcomes for organisations (Lave \& Wenger, 1991). Based on these claimed benefits, organisations increasingly began to create CoPs. Initially, these CoPs started as environments 
to stimulate knowledge management within organisations. To stimulate knowledge management within CoPs, individuals organize their own conferences, presentations, workshops, seminars and other opportunities for members to exchange ideas, build skills, and develop their own professional networks (Wenger, 2000). Developments in Information and Communication Technologies (ICT) have led to an massive growth in online options for knowledge management, collaboration, social bonding and professional learning in general (Vries, 2003). To take full advantage of these (new) opportunities, an increasing number of organisations opt for the purchase of a licence for an online community platform. Professionals use this online community software for knowledge management, collaboration and other social purposes (Jiménez-Zarco et al., 20l4).

\subsubsection{Community development}

Community development, evolution and lifecycles are attracting much interest among academics, consultants and commercial organisations (Wenger, McDermott \& Snyder, 2002;Wenger, 1998). In the literature, two types of stage models are found: life cycle models (from beginning to end) and evolution models (from low to high levels of maturity) (Andriessen, 2005). Wenger (1998) and McDermott (2000) have created a life cycle model to understand the development of CoPs; these models are mostly based on observations, interpretations and expertise. Gongla \& Rizzuto (200I) introduced the evolution model, which describes how communities transform themselves, becoming more capable at each stage. These lifecycle and evolution models are predominantly focused on ideal situations, which show an upward trend. However, reality is more volatile, and most CoPs develop in a less predictable direction. Members loose interest or have a lack of time, managers decide to refocus their knowledge management activities or technology does not meet the requirements set during initiation (Ardichvili et al., 2003)—all of these are reasons why communal activities decrease. On the other hand, if the technology and the community purposes are adopted and adapted in the organisational context (Rowe, Fernandez, Angeletou, \& Alani, 2012) and if the motives and motivations of the individual members are positive (Ardichvili et al., 2003), the community activities almost automatically increase. In general, one of the commonly agreed-upon facts is that communities have a development path, which means that they emerge, grow and have a life span. These life spans are longer for some communities than for others. In this research, the development of CoPs is studied by using the usage data derived from the online databases of CoPs, defined as webbased social interaction data. This data makes it possible to observe, analyse and test individual and group behaviour. Due to the exploratory nature of this dissertation, the existing theories and literature are used as inputs. However, our primary goal is not to test one of the existing lifecycles or maturity models but to generate insights into existing social behaviour patterns identifiable in each CoP. However, these social behaviour patterns can be placed on the path of community development, which is comparable to lifecycle or maturity models.

\subsubsection{Community Member Roles}

Identifying social behaviour patterns is the first step, and the second step is interpreting these patterns-namely, determining what affects the ups and downs in the development of a CoP. It is assumed that the community members and their type of behaviour largely contribute to the developments in the CoP, including the direction of these developments. Community members are the lifeblood of online communal spaces (Cowan \& Menchaca, 2014). The way community participants move through a community largely differs by individual. A CoP often starts because of the enthusiasm of some participants. Wenger (2006, p.2) compares 
the enthusiasm of these few participants with the start of a relationship: "It has the same mixture of excitement and apprehension, the relationship is palpable; it feeds the imagination, but the prospects remain unclear". In addition to this limited group of enthusiasts, the vast majority consists of participants who can be characterized by their legitimate peripheral form of social behaviour. Lave \&Wenger (199I) describe this form of social behaviour as the process where newcomers start in the peripheral area of a CoP and become, at changing paces, more experienced members and then eventually become old timers. In peripheral activities, new community members become familiar with the tasks, language, and structure and organizing principles of the community. This group, which forms a substantial part of the whole community, develops in different directions, resulting in a variety of member types. The author's research perspective largely determines how the roles or member types are identified and defined, but in general, three recurring member types with varying titles can be identified: the community leader, the contributor and the information absorber (Drew, McCallum \& Roggenhofer, 2016). Many CoPs start with a formally assigned community leader, and this leader intends to stimulate others to become active community members. The resulting active group can be defined as the contributors; these group members share their knowledge, experiences and best practices. HOwever, most of these studies look at developments and the role of community members in it from a helicopter perspective. By looking at existing structures and developments, insight is often gained into a greater variety of community members. Purely focusing on the group of contributors show several other types, for example, posters (Drew \& McCallum, 2016), commenters (Miller \& Proctor, 2016), chatters (Purvis et al., 200I), advisers (Wellman, 1997), appreciators (Bista et al., 20I2) and networkers (Nardi, Whittaker, \& Schwarz, 2002). This last group consists of inactive participants (Iriberri \& Leroy, 2009). This group has registered for a CoP but then shows little or no activity. In summary, the focus in this study is on identifying the following member roles within the CoPs: information absorber, inactives, commenters, posters, networkers, appreciators, chatters, advisers, and community leaders.

\subsection{Method}

In this chapter, different complementary methods and techniques are used to show and evaluate community developments in CoPs. The underlying approaches form an important component of the community monitoring and evaluation methodology (CMEM), as described in Chapter 5. Following the subdivision of Banerjee, Bandyopadhyay, \& Acharya (2013)-introduced in Chapter 5-the methods used in this chapter are related to the analysis of descriptive and prescriptive community analytics.

A typical data-driven approach has been applied (Figure 5.2) with data science methods to gain insight into community development. Data science has been used as a general term to refer to a wide set of skills and practices required to operate in the big data area (Doran, 20I5). In this inductive type of research, we conducted a web usage mining approach. Web usage mining is an area of web mining that deals with techniques to discover usage patterns in web data to improve our understanding of (online) behaviour (Han \& Tang, 20I5). This approach—described in Chapter 5-describes the process from data gathering to strategy development. In this study, the four central cases are supplemented with three other comparable cases that also use the same type of technology as the main four cases. These cases are selected after interviews with the respective community managers, based on these interviews it can be concluded that these initiatives fit within the definition of a CoP as used in this study. However, after consultation with the community managers, it was decided to let these cases only participate in this part of the study. Sending out a questionnaire such as in the previous chapter could not proceed 
because the community members had recently taken part in another study. This fact could considerably influence the results. In total, we extracted all online social interactions of the community members in seven Dutch CoPs. The key motivation for the decision to focus on the entire network instead of individual sub-communities is when using the entire network as a focus area, the amount of data increases and the amount of bias will be reduced due to the volume (Vaudel et al., 2015). In Table 9. I, a brief overview of the statistics of all CoPs is given.

Each of these seven CoPs consists of a plurality of domain-specific groups; these groups are called sub-communities. The fact that the CoPs in this study are all equipped with the same technology ensures that the data resulting from the platform have the same structure for each organisation and ensures that each CoP has an equal chance that a certain form of interaction occurs more frequently. The choice of the type of technology in combination with the way in which the community managers describe their online environment led us to decide to include these seven cases in the study. The use of multiple cases enabled us to replicate and compare the results in one case with another.

The first step in the data-driven process is to understand the businesses in these cases. Good insight is required into the background of the organisation, the objectives they have with the CoPs, etcetera. Table 9.I briefly shows the composition of each composition, unfortunately insight into the explicit objectives of the last three cases is missing. Data gathering, data processing and data mining are the next steps in the data driven process and have been conducted by using Microsoft Excel 20 I 3, MySQLWorkbench, IBM SPSS Statistics 23 and Tableau Software I0.I and forms in conjunction with the subsequent steps pattern evaluation, knowledge presentation, and strategic decision-making the basis for the following paragraphs.

Table 9.I

Description of the CoPs in the first 14 months

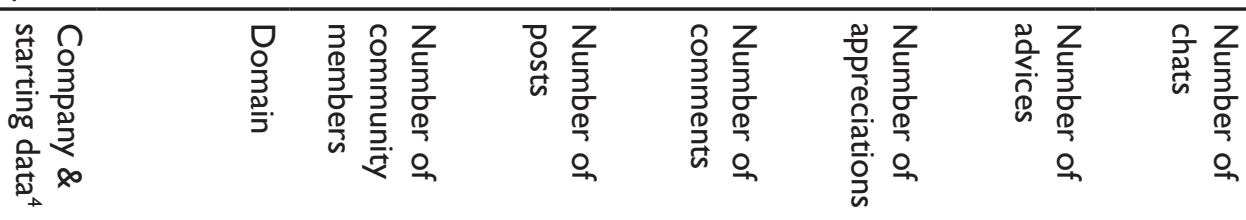

$\begin{array}{llllllll}\begin{array}{l}\text { Company } \\ \text { A }(2013)\end{array} & \begin{array}{l}\text { Healthcare } \\ \text { (internal) }\end{array} & 1305 & 405 & 517 & 111 & 79 & 397 \\ \begin{array}{l}\text { Company } \\ \text { B }(2013)\end{array} & \begin{array}{l}\text { Youth Care } \\ \text { (internal) }\end{array} & 1228 & 3019 & 4201 & 1587 & 470 & 1416 \\ \text { Company } & \begin{array}{l}\text { Disability } \\ \text { nursing } \\ \text { (internal) }\end{array} & 397 & 1992 & 1562 & 1212 & 213 & 920 \\ & & & & & & & \\ \text { Company } & \begin{array}{l}\text { Finance } \\ \text { (internal) }\end{array} & 330 & 2722 & 2131 & 732 & 670 & 1414 \\ \text { D (2014) } & & & & & & \end{array}$

${ }^{4}$ The cases A, B, C and D are also central cases in the previous and coming chapters. The cases D, E and F are new cases. 


\begin{tabular}{|c|c|c|c|c|c|c|c|}
\hline 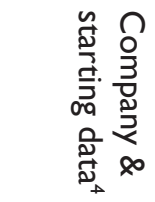 & $\begin{array}{l}\text { ○ } \\
\frac{0}{3} \\
\frac{0}{3}\end{array}$ & 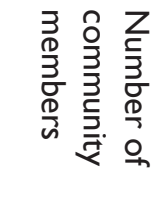 & 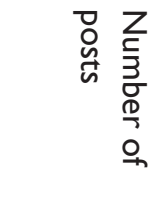 & 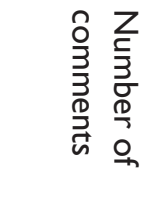 & 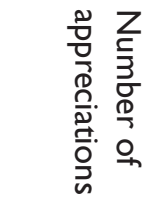 & 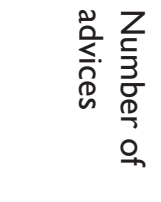 & 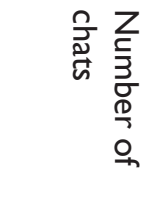 \\
\hline $\begin{array}{l}\text { Company } \\
\text { E (2008) }\end{array}$ & $\begin{array}{l}\text { Food } \\
\text { industry } \\
\text { (internal) }\end{array}$ & 248 & 515 & 184 & 21 & 0 & 212 \\
\hline $\begin{array}{l}\text { Company } \\
F(2016)\end{array}$ & $\begin{array}{l}\text { Care for } \\
\text { the elderly } \\
\text { (internal } \\
\text { and } \\
\text { external) }\end{array}$ & 649 & 243 & 566 & 837 & 332 & $|23|$ \\
\hline $\begin{array}{l}\text { Company } \\
\text { G (20I2) }\end{array}$ & $\begin{array}{l}\text { Social care } \\
\text { (Internal } \\
\text { and } \\
\text { external) }\end{array}$ & 525 & 679 & 1428 & 402 & 431 & 2448 \\
\hline
\end{tabular}

\subsection{Data collection, gathering, processing and mining}

In this study, web-based social interaction data (e.g., messages, comments and likes) from online community software form an important resource for obtaining objective and real-time information about the community behaviour of individuals. The web-based social interaction data were collected, stored and processed in agreement with the participating seven organisations. These seven CoPs were recruited based on three criteria: (I) all CoPs must use the same technology to ensure that the data and subsequent analysis are reliable, (2) each CoP must focus on collaboratively improving practice and (3) each CoP must have been established for at least 12 months.

By using data mining techniques, the raw web-based social interaction data were processed by searching for patterns and relationships that are of significant value for describing, explaining, predicting and controlling the development of CoP behaviour.The web-based social interaction data were stored in a MySQL database and prepared for research purposes.

After completing the registration for a CoP, each member has automatically created a profile in the online environment, this also applies to the participants in this study.A profile gives them the opportunity to start different types of social interaction. In this dissertation, seven types of social interaction are distinguished: (I) visiting the community, (2) creating a post, (3) sending a comment to a post, (4) recommending a post to another community member, (5) appreciating the post of another community member, (6) following another community member's social interaction behaviour, and (7) chatting on a one-to-one basis with other community members. In the following paragraphs, these types of social interactions have a major role in achieving 
the three objectives: (I) identify and formalize the developments in the CoPs (2) identify community member roles in CoPs, and (3) analyse relationships between the developments and the distributions of member types.

\subsubsection{Methods to get insight into community development}

Most CoPs are organized with the idea that they should contribute to the improvement of current organisational or disciplinary practices. Not all CoPs perfectly realize this objective; some fail in the adoption phase, some in the implementation phase and others in the use phase. Creating a CoP that evolves in a preferred direction requires a clear vision, specific objectives and a great deal of time investment. In this part of the study, the development processes of seven different CoPs are analysed in detail. Some CoPs had already existed for more than nine years, while one CoP had started only 14 months prior to the study. To improve the generalizability of the results, the focus is on the first 14 months of all CoPs. These 14 months are considered to be the start-up phase of the CoP, and the development processes of the CoPs within these 14 months are analysed in-depth.

We evaluate this development by focusing on different community development patterns that are unique to each CoP and the intensity of the social interactions over the duration of the CoP that lead to these developments. To improve the validity of the results, we decided to standardize the scores. Standardized scores summarize a score's relative position in a group; a group in this study consists of all participants in each CoP.The general accepted approach to determine the standardized scores is to use the z-scores However, a z-score describes an individual's score relative to the group and is based on the group mean. In this study, the mean score of each group differs by form of interaction and case. If the z-scores were used, a distorted view of the situation would be given. For that reason, a specific approach to standardize the scores for each CoP has been developed. In this approach, the total number of times a certain type of social interaction occurs during a given month in one CoP is divided by the total number of all types of social interaction during the first 14 months of that community. The resulting score is multiplied by the total score of all social interactions in all CoPs for one month. In this case, five of the seven types of social interaction were included in the calculations. The forms visiting the community and following another community member's social interaction behaviour are not included in the calculations since this part of the research focuses on individual contributions to the CoP which is of benefit for the group. This approach ensures that we obtain the most reliable picture of the relative intensity of each form of interaction in the CoPs. This results in a valid approach for comparing all scores in the CoPs.

\subsubsection{Methods to get insight into community member roles}

This part of the study is an exploratory attempt to identify and analyse community member roles within a CoP based on the particular form(s) of social interaction they exhibit. Everyone behaves in a unique way, which can vary from knowledge gaining to knowledge sharing to discussing. In this part of the study, we focus on identifying unique member roles, as well as identifying which member roles are the most common, and in which phases we see these member roles the most. However, since we use large amounts of data from seven CoPs, the external validity also automatically increases.

In the process of identifying community member roles, we have chosen to standardize the values associated with each form of interaction. Since each CoP in this study started at different 
times, the values fluctuate notably. However, to improve our understanding of the activities and development, it is crucial to standardize the values to make them comparable.The normalization process has resulted in values between zero and one, and the min-max normalization has been used to achieve this (Jiménez-Zarco, González-González, Saigí-Rubió \& Torrent-Sellens, 20I4).

$$
\mathrm{z}^{\mathrm{i}}=\frac{\mathrm{x}^{i}-\min (\mathrm{x})}{\max (\mathrm{x})-\boldsymbol{\operatorname { m i n }}(\mathrm{x})}
$$

One normally uses z-scores to standardize the values on certain variables. However, inactive participants can -in that case-obtain a relatively higher score on one form of interaction than on another even when the score on both forms of interaction is zero. The resulting scores between one and zero have been weighted to make a clear distinction between cognitive stressing activities. The value of 'visiting the CoP' is, in that case, devalued so that it can be seen in proportion.

\subsection{Results}

The results are evaluated as follows: in the first step, the community development patterns are described as an indication of the developments within all CoPs. These results are based on the standardized scores of each CoP.The second step is to determine what member roles exist in a CoP and the third step consists of a member role distribution in the CoPs.

\subsection{Community development}

There is no fixed answer to the question how CoPs evolve over time, as this differ by situation. The way a CoP starts also differs with each situation. In some CoPs, community members actively and enthusiastically start conversing with others, while in other communities, a wellconsidered, gradual implementation trajectory is being carried out in which only a pre-selected group of community members are invited to participate in the CoP. In this sophisticated implementation strategy, the focus is on implementing the CoP in the work processes of the professionals. For this study, the web-based social interaction data form the basis for identifying overarching patterns that describe the general development of a CoP.

Figure 9.I gives an overview of the total normalized score of all activities in the seven CoPs. The normalized score ensures that all organisations are treated equally and that outliers in the differences between the organisations become proportional. However, especially when the community development patterns of all CoPs are analysed in one graph, it is important to evaluate these patterns with caution. Extreme outliers cannot be fully normalized, which means they can have a significant impact on community development patterns. Generally, each CoP shows a considerable increase in the activities after a run-up of a month or sometimes a few months; after this sharp rise, a slowdown occurs. Thereafter, the social activities in most CoPs gradually increase in size. The CoPs are recognized and accepted by a growing number of professionals. To understand the background of these developments, it has been decided to extract the types of social interaction that determine this general development. In Figure 9.2, the types of social interaction are distributed in the first 14 months of the seven CoPs, defined as the start-up phase. 


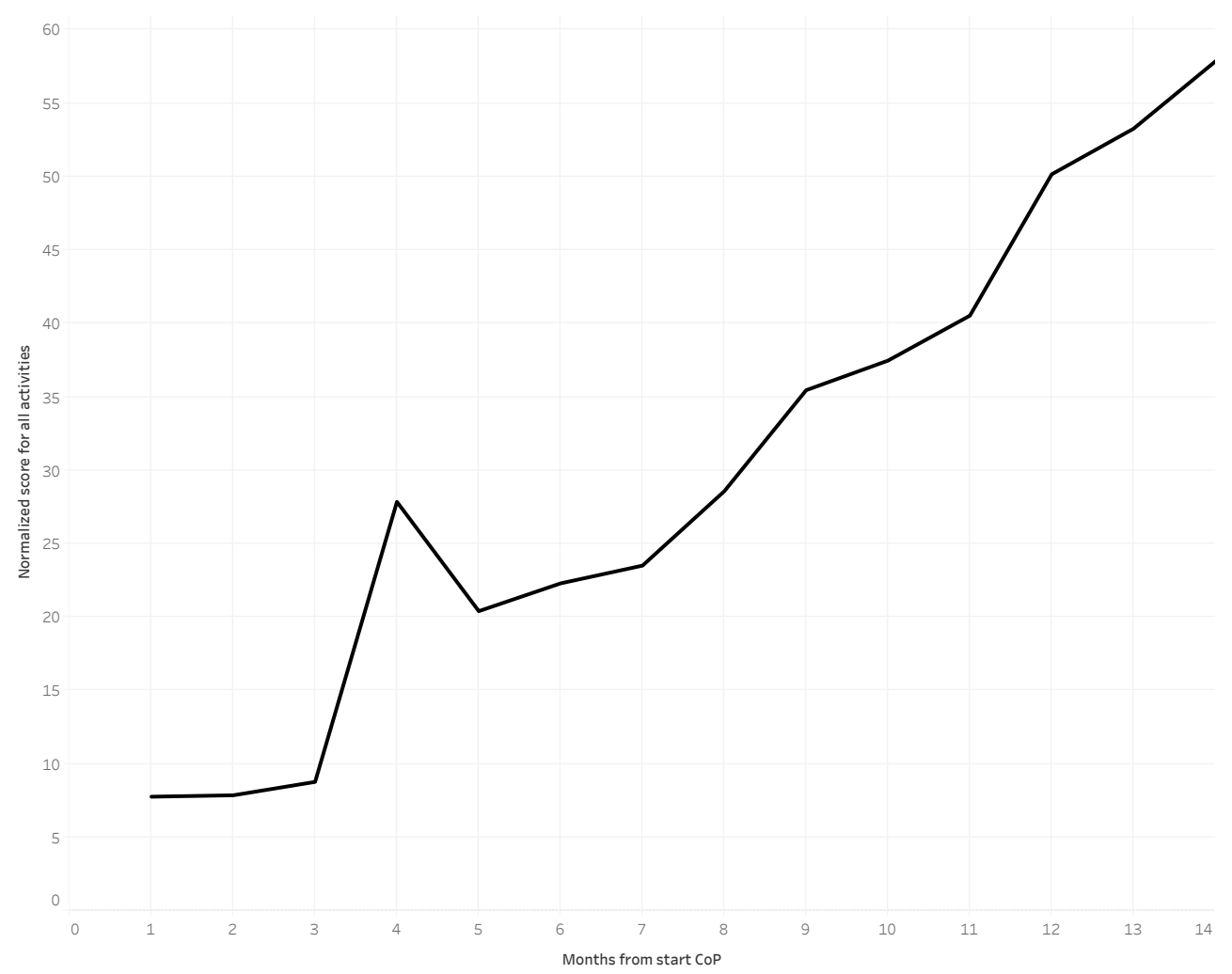

Figure 9.I. Start-up phase: Normalized scores of the number of social interactions in the first 14 months of 7 CoPs

What stands out in the overall development of social interaction in CoPs shown in Figure 9.I is the general pattern of a sharp peak after a couple of months, a rapid decrease following this peak and the continuous growth after this dip. By researching and analysing the establishment of these developments, it is possible to completely map the types of social interaction that form the basis of the CoP.The types of social interactions that determine the development of social interactions in a CoP are shown in Figure 9.2. The overview is constructed by the normalized scores of these active forms of communication. Over time, some remarkable patterns can be identified. One salient trend is the clear increase in one-to-one chats after four months. Some plausible explanations for this might be the need for informal conversations (e.g. to interact, gossip and be in contact with other colleagues), but also the fact that some members actively encourage others to participate in the CoP via a one-to-one message is possible. The result is an increase in almost all other types of activity: creating posts, commenting on posts and appreciating posts. 


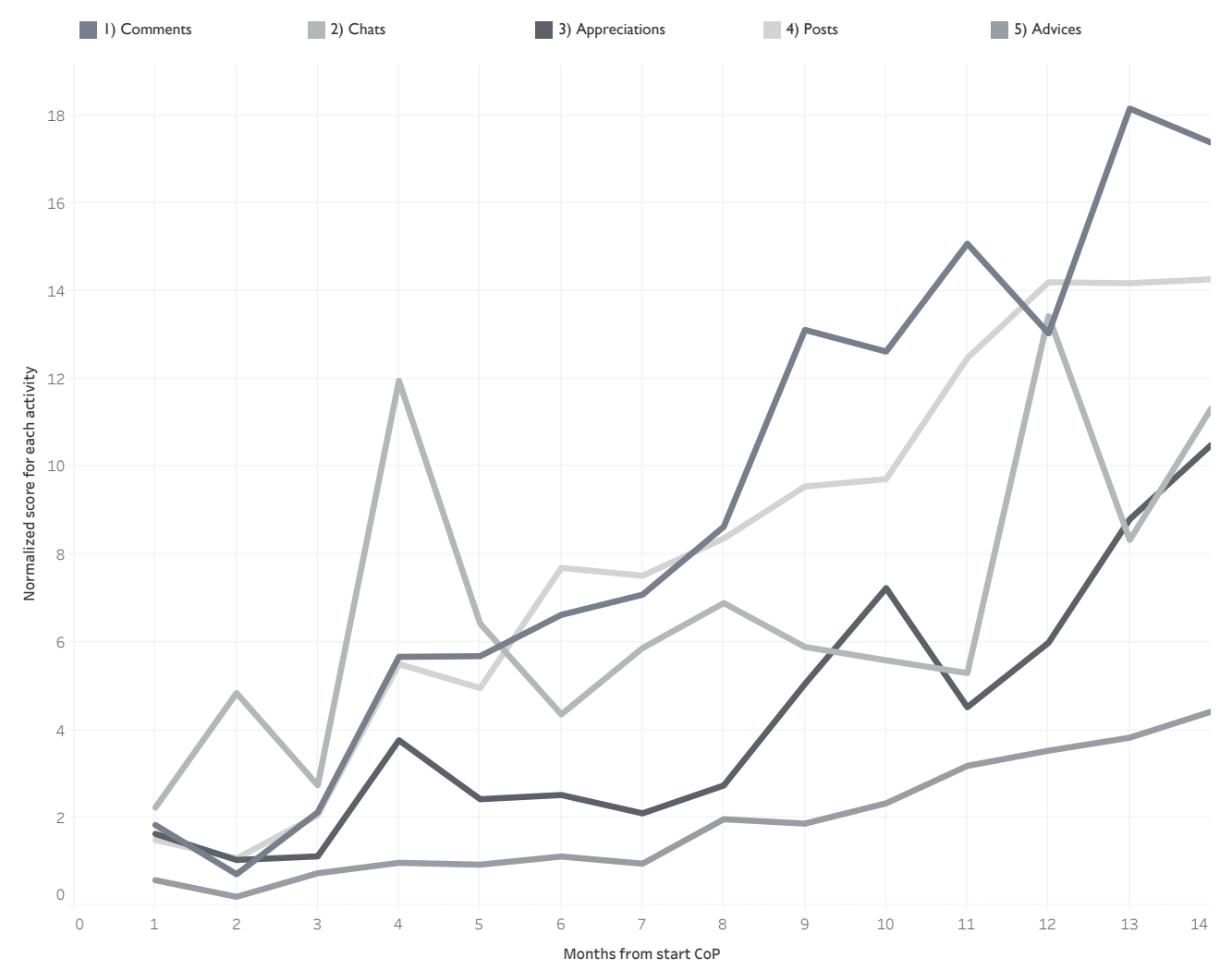

Figure 9.2. Start-up phase: community development patterns for the seven cases

Figures 9.3 to 9.9 show the visualization of typical community development patterns within the seven cases. By evaluating the development of the CoPs based on the implicit goals that are (possibly) set within the CoPs, it is possible to give an indication of the success of these CoPs. From the perspective of a community of practice, this research uses a highly interactive group of participants who want to advance organisational practice by means of a variety of social interaction types as a measurement for a successful CoP. In this study, the goals defined in Chapter 3 are used to measure the realization of the implicit goals. These goals are: stimulating the collective learning, innovating and improving practice, making the knowledge management more efficient, create tangible, measurable and value-adding benefits for the organisation, and increasing the opportunities for professional networking.

The goals set in Company A (Chapter 4) are to stimulate the collective learning, innovating and improving practice and to increase the opportunities for professional networking. The results indicate that the CoP (Figure 9.3) is heading towards realizing these objectives, since there is a steep increase in the social interactions after 8 months. However, in general the amount of social interactions is comparable in quantity, so this implies that the amount of posts is almost similar as the amount comments. The developments thus display a knowledge-centred pattern, but the last two months show signs of a community-centred pattern, with larger differences between the amount of comments and posts. A typical community-centred development is characterized by the sharp increase in the number of social interactions, but also in the parallel increase in these social interactions. Not one of the types of social interactions is increasing 
strongly, but a significant part of these forms. In contrast to the other typical community-centred development patterns in the other cases, those in company A show a longer run-in-period. This also has something to do with the implementation strategy in Company A. The company has as described in Chapter 4 (large hospital) - been gradually implemented within the organisation, according to the diffusion approach. The consequence of this is that the intensity of social behaviour starts later.

The CoP in company B (Figure 9.4) shows a typical community-centred development, all types of social interaction increase almost simultaneously. The organisational objectives set by this organisation are to improve the collective learning, more efficient knowledge management, and increasing the opportunities for professional networking. The CoP shows highly variable success as witnessed by the large differences in intensity of social interactions during the starting phase of the CoP. The number of posts in any case indicate that the knowledge management is at an adequate level. The collective learning symbolized in the amount of posts, comments, appreciations and advices are also promising. Achieving the latter implicit goal - increasing the opportunities for professional networking - also looks promising, given the considerable amount of chat conversations within the CoP. In general, the CoPs a typical community-centred pattern which indicates that the community is increasingly being accepted by its participants and increasingly integrated into daily work practice.

Considering primarily the types of social interaction in the CoP of Company C (Figure 9.5), a somewhat similar pattern is visible as in the CoPs of organisations $A$ and $B$. The objectives that appear to be set by Company $\mathrm{C}$ - defined in Chapter 4 - are improving the collective learning and ensuring more efficient knowledge management. The trends show a promising increase in all types of social interaction. However, after further zooming in on developments within this community, the picture emerges that - not including the outlier in month 4 - the dominant type of social interaction is the posting of messages. This might indicate a more knowledge-centred pattern, with social behaviour primarily focused on publishing messages that should be read by others. The data further show a clear peak in attention for the CoP after four months. The amount of chats, posts, comments and appreciations rose sharply in that month. After this, a very strong decrease can be observed, after which the developments of almost every type of social interaction - with the exception of advices - gradually increase.

Company D (Figure 9.6) also show a development which can be characterized as a knowledgecentred pattern. The goals set by this organisation (Chapter 4) are to improve the collective learning and to make knowledge management more efficient. The results show a knowledgecentred pattern development, which indicate that publishing posts in the CoP is the primary type of social interaction. This result is in any case an indication that knowledge management is organized more efficiently. However, the other types of social interaction lag the sharing of knowledge, which forms an indication that improving the collective learning - in the starting phase - is not yet achieved. Only in a few locations (months 4, 7 and II), the more communitycentred types of social interaction shown.

For the companies E, F and G, the goals set with the CoP have not been explicitly mentioned by the community managers, so that implicit goals are used for these cases. It seems that Company E (Figure 9.7) primarily focuses on developing an adequate knowledge management basis, for example, improving collective learning, innovating, or improving professional networking do not seem to be an issue. The first 14 months show a somewhat negative trend, particularly in the intensity of social interactions. The amount of social interactions is quite low, as evidenced 
by the values on the normalized scale and during the timespan of the 14 months, the amount of social interactions is only decreasing. In general, some of the community members only use it to donate some knowledge, but at a minimal level. Community management seems to have carried out several interventions to activate community members, for example in the form of personal chats and public posts, nevertheless there was a sharp decline in the activity of the participants. In addition, the intensity of interactions in this CoP is at all lower than in the other companies, as can be seen in the normalized scores. The patterns in this CoP indicate that the implicit objectives will not be achieved during the sample of this study.

The CoP in both organisation F and $G$ contrasts with the other communities since these are not primarily focused on communication within organisations (intra-organisational), but also on organisations with a focus outside the boundaries of their own organisation (interorganisational). The developments in the CoP of Company $\mathrm{F}$ show a development that points to an implicit goal that focuses on improving professional networks. The pattern that can be recognized in this CoP shows a more dialogue-centred development (Figure 9.8) in which participants predominantly have one-to-one chats. This could imply that the members in this community mainly participate in the CoP for consulting purposes and to develop and/or strengthen the one-to-one relationships between participants. This CoP is an interdisciplinary network of healthcare professionals represented in different organisations. Therefore, members often consult colleagues/peers from other organisations in case issues transcend business and organisations.

The CoP initiated in organisation G shows a typical community-centred development pattern (Figure 9.9). Whereas in other CoPs, the posts are overrepresented - which indicates a knowledge-centred pattern - the emphasis here is on responding to posts from the very beginning and to chat on a one-to-one basis. The relatively high scores on the personal chats in this CoP can be explained by the fact that the community members are highly centralized, the come from all kinds of organisations that operate from different locations. It is therefore very likely that the participants are particularly looking for relevant professionals who contribute to their own professional network. The results indicate that the following implicit goals are set and are on track to be achieved: improving the continuous learning of individuals, of organizing knowledge management more efficiently and of an improvement of the professional networking activities between community members.

One of the similarities that emerges clearly is a strong increase in the number of social interactions after a short - or in some cases, a somewhat longer - starting time. After the first rising line, a decent valley usually follows, which is, for many community managers or information specialists a signal to organise interventions to stimulate the activity (again). 


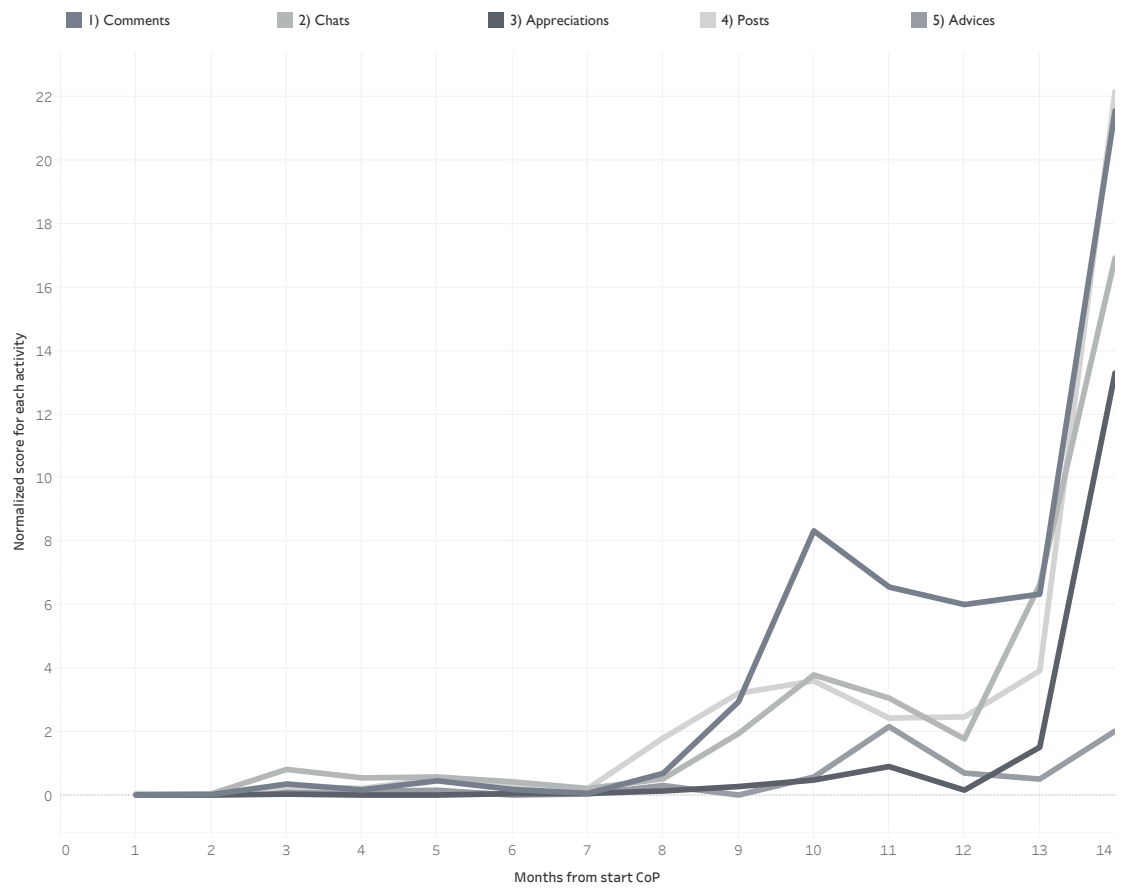

Figure 9.3. Knowledge-centred development in Company A

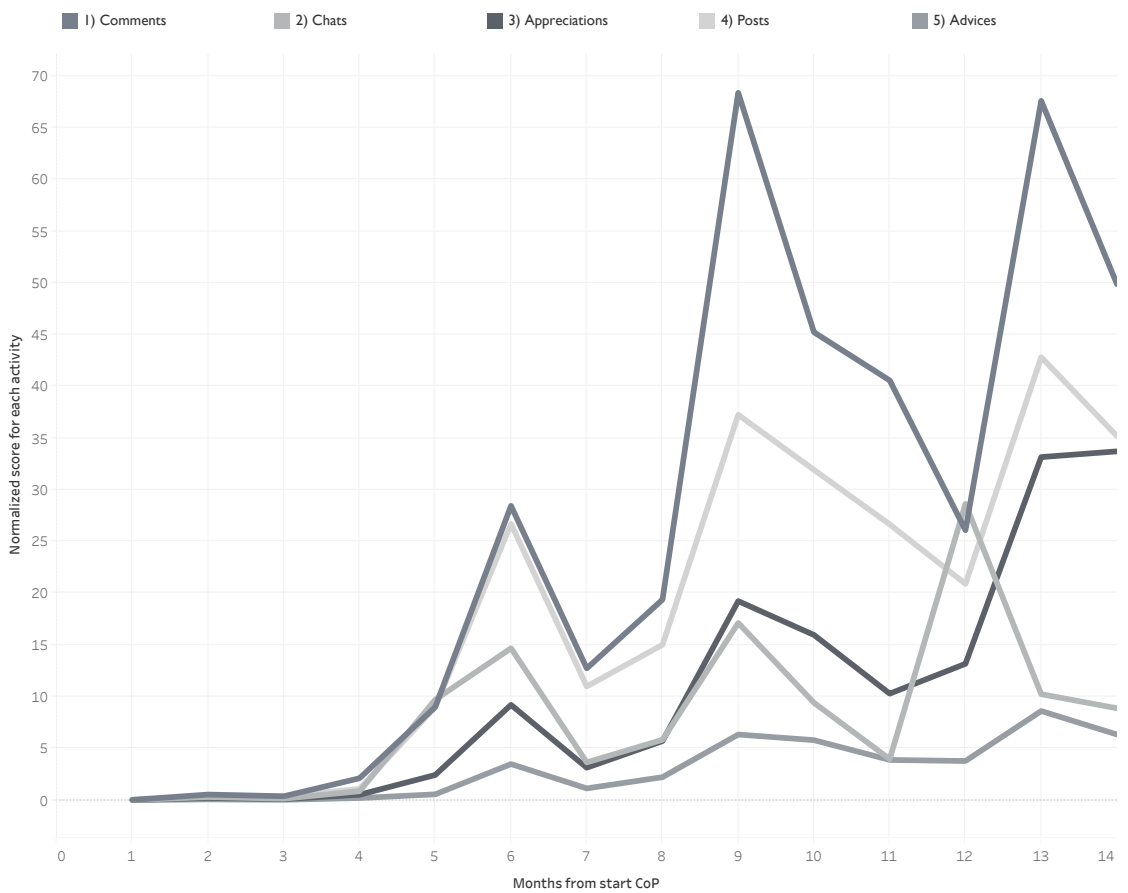

Figure 9.4. Community-centred development in Company B 
1) Comments

3) Appreciations

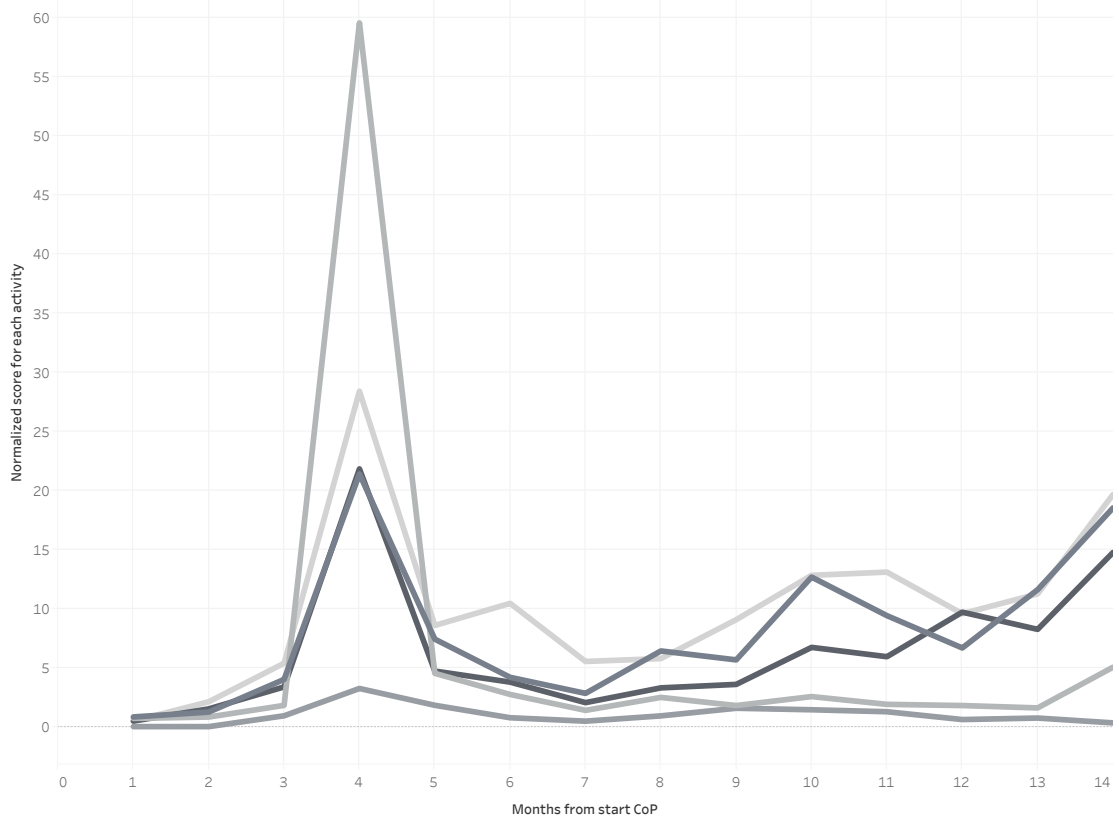

Figure 9.5. Knowledge-centred development in Company C

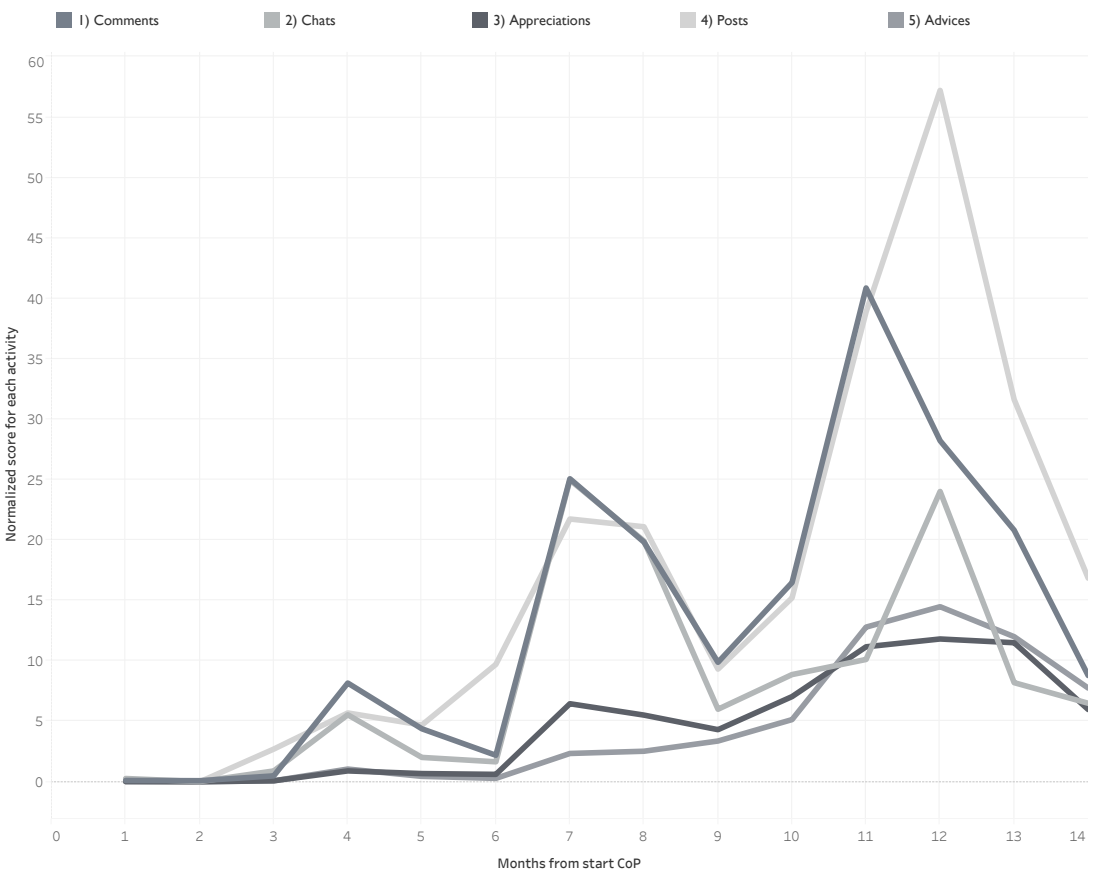

Figure 9.6. Knowledge-centred development in Company D 


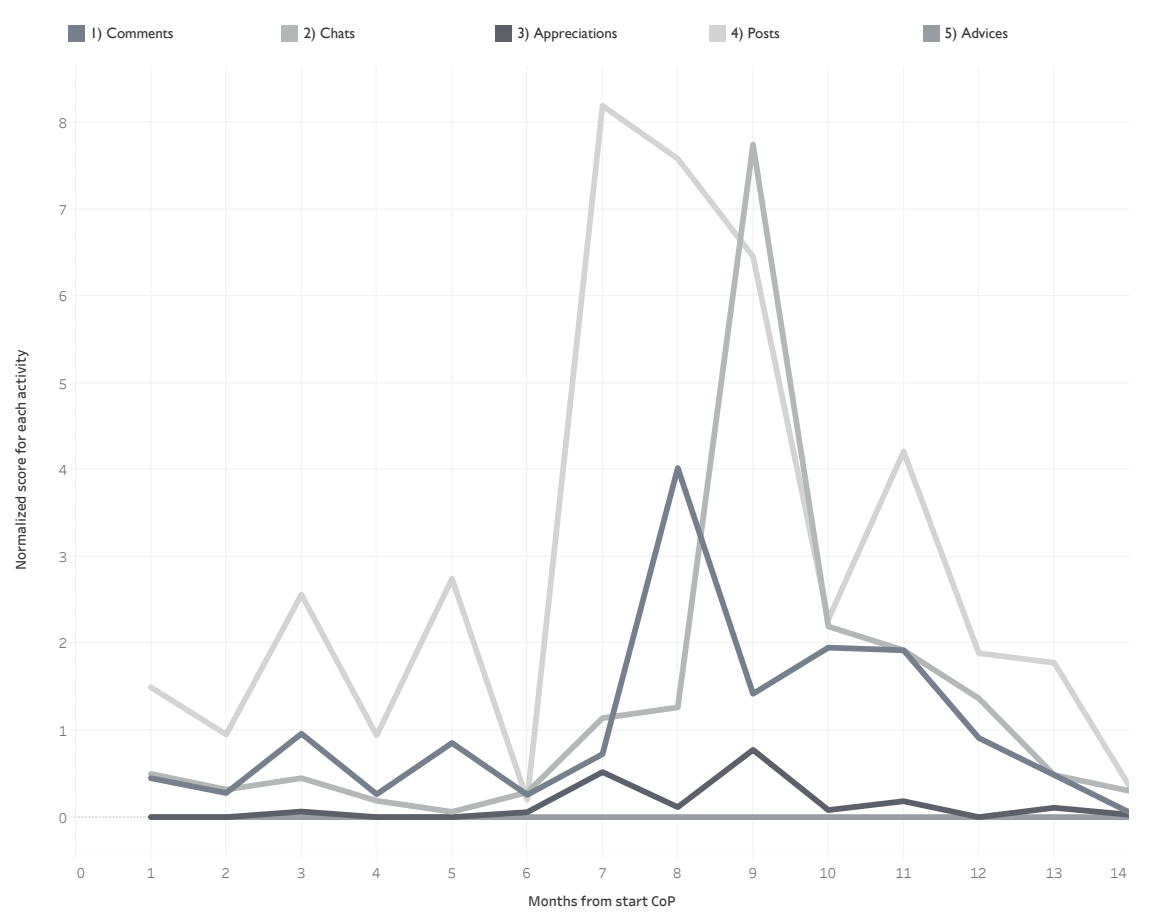

Figure 9.7. Knowledge-centred development in Company E

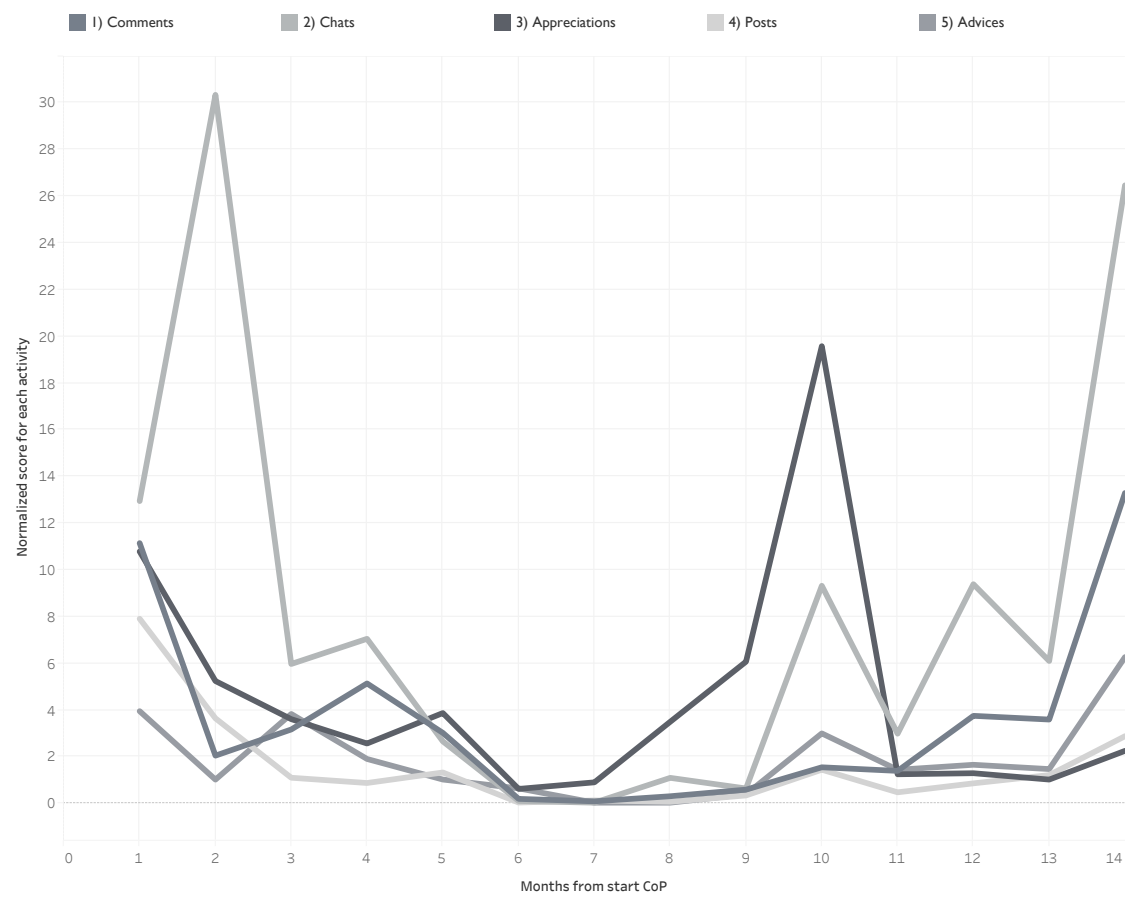

Figure 9.8. Dialogue-centred development in Company F 


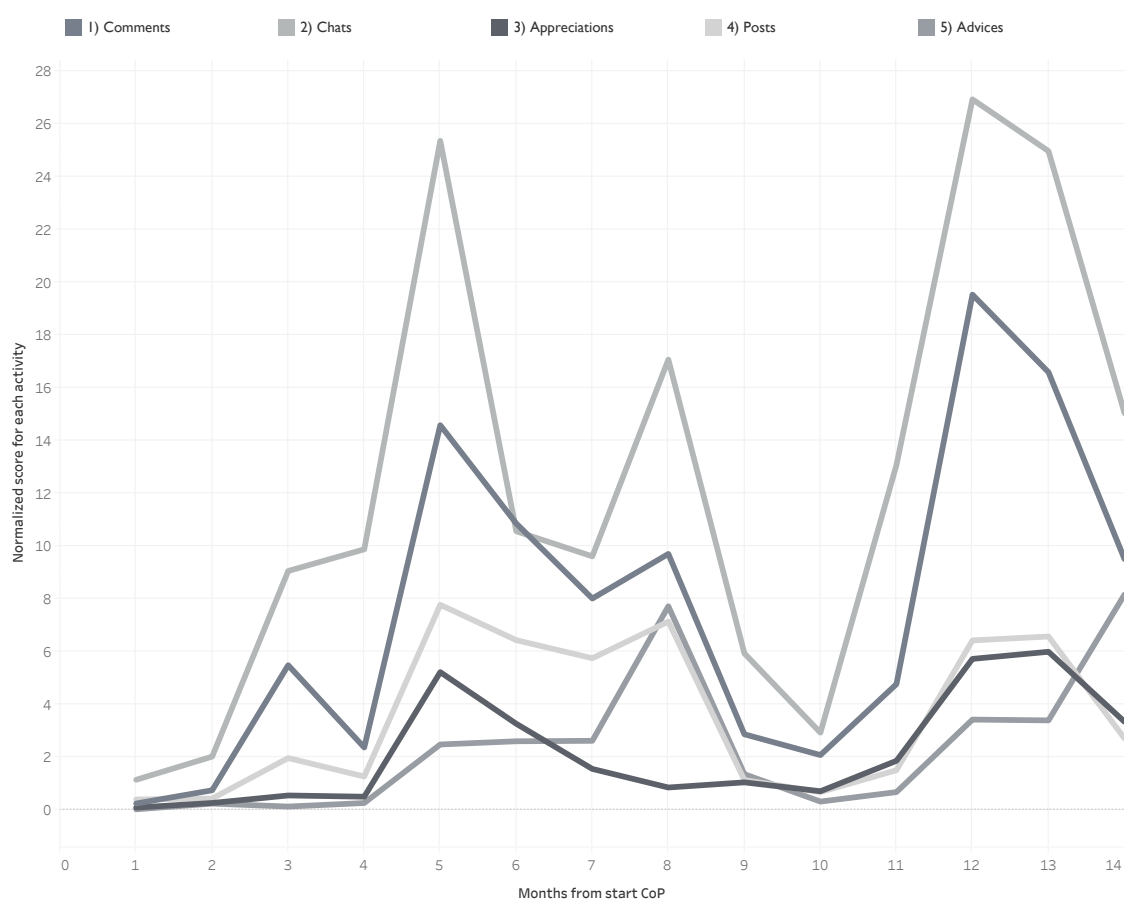

Figure 9.9. Community-centred development in Company G

\subsubsection{Community member roles}

As a community manager it is valuable to know what types of members participate in the community. Focusing on the behaviour of the community members offers opportunities to recognize patterns that depict certain types of community members. Each participant in a community takes part in different way; some are active message posters; others choose specifically to engage in discussion or dialogue, and there are also participants who combine all of these types of social interaction. Building and maintaining a modern virtual CoP means generating motivation and enthusiasm among new and existing community members to continuously optimize the knowledge and innovation level within the CoP. However, the enthusiasm of community members differs greatly by person. Some community members actively participate in the community from the beginning by sharing knowledge, connecting people, and discussing relevant topics. Others are primarily keen to fulfil their own information needs and thereby attach less value to sharing their own knowledge and experiences.

The constellation of individual types of social interaction from an individual user can be recognized as that user's type of membership. Since this study consists of seven CoPs in just as many organisations, the intensity of social behaviour largely differs. Some CoPs began nine years ago, while others started just one year ago. To reduce the skewness in the data, the numbers for each form of interaction have been normalized for each individual community member. The normalized scores give an indication of the relative activity compared to the other CoPs instead of the absolute activity that would only be relevant for one CoP. 
Since each interaction within the system counts for an increase of one for that form of interaction, visiting the CoP will for everyone, be the dominant form of interaction. To ensure that the intensity of each type of social interaction is in proportion, we have weighted visiting the cognitively less intensive type of social interaction - by a coefficient of ten. Otherwise each member would be just an information absorber instead of a more active member.

The normalized and weighted scores provide an indication of those activities in which the community member is relatively the most active. Based on these scores and clusters of these scores, we categorized these members into member roles. In Table 9.2, these member roles are defined, and insight is given into the distribution of these member roles across all communities (Figure 9.10). Only a minimal number of participants belong to the group of community leaders. This group of members is the driving force of every community, and they are continuously keen on stimulating others to participate. They act as the flywheels or catalysts of the entire community and often have the formal role of a community manager or an ambassador. Most of the participants belong to the category of information absorbers or inactive users. Information absorbers rarely contribute to the CoP but frequently visit the CoP to acquire knowledge or information on the experiences of others that might be beneficial for their own daily work. It is for that reason very likely that these participants apply the acquired knowledge in their own practical situations. Another substantial group consists of inactive users. Members in this group have created an account, but only visit the CoP sporadically. It is very likely that this group of participants do not identify with the group identity or the set objectives and directions. These members are often forced by management to register for the CoP. Other active members in the CoP are posters $(5.0 \%)$, commenters $(6.2 \%)$, appreciators $(4.2 \%)$, advisers $(0.5 \%)$, chatters (3.1\%) and networkers (5.0\%).

Table 9.2

Description and operationalization of the member roles in 7 cases

\section{Member type Description Operationalization Percentage of total population in CoPs}

$\begin{array}{lll}\begin{array}{l}\text { Information } \\ \text { absorber }\end{array} & \begin{array}{l}\text { A person who } \\ \text { regularly visits } \\ \text { the CoP without } \\ \text { making any } \\ \text { contributions to it. }\end{array} & \begin{array}{l}\text { Relatively high scores } \\ \text { on the "visit" form of } \\ \text { interaction; relatively low } \\ \text { scores on the other forms } \\ \text { of interaction. }\end{array} \\ \text { Inactives } & \begin{array}{l}\text { A person who } \\ \text { registered for the } \\ \text { community but } \\ \text { (almost) never } \\ \text { came back. }\end{array} & \begin{array}{l}\text { Low scores on all forms of } \\ \text { interaction. }\end{array} \\ & \begin{array}{l}\text { A person } \\ \text { who regularly } \\ \text { comments on the } \\ \text { contributions of } \\ \text { others. }\end{array} & \begin{array}{l}\text { Relatively high scores on } \\ \text { the active and passive } \\ \text { "comments" forms of } \\ \text { interaction; relatively low } \\ \text { scores on the other forms } \\ \text { of interaction. }\end{array} \\ & & 6.2 \%\end{array}$




\begin{tabular}{|c|c|c|c|}
\hline Member type & Description & Operationalization & $\begin{array}{l}\text { Percentage of total } \\
\text { population in CoPs }\end{array}$ \\
\hline Posters & $\begin{array}{l}\text { A person } \\
\text { who regularly } \\
\text { contributes to } \\
\text { the CoP with new } \\
\text { posts. }\end{array}$ & $\begin{array}{l}\text { Relatively high scores } \\
\text { on the active "creating } \\
\text { posts" form of interaction; } \\
\text { relatively low scores on the } \\
\text { other forms of interaction. }\end{array}$ & $5.0 \%$ \\
\hline Networkers & $\begin{array}{l}\text { A person who uses } \\
\text { the CoP mainly to } \\
\text { follow (interesting) } \\
\text { colleagues. }\end{array}$ & $\begin{array}{l}\text { Relatively high scores on } \\
\text { the active 'following' form } \\
\text { of interaction; relatively low } \\
\text { scores on the other forms } \\
\text { of interaction. }\end{array}$ & $5.0 \%$ \\
\hline Appreciators & $\begin{array}{l}\text { A person who } \\
\text { reads posts } \\
\text { or comments } \\
\text { and expresses } \\
\text { appreciation } \\
\text { for these } \\
\text { contributions. }\end{array}$ & $\begin{array}{l}\text { Relatively high scores on } \\
\text { the active "appreciating } \\
\text { others' contributions" form } \\
\text { of interaction; relatively low } \\
\text { scores on the other forms } \\
\text { of interaction. }\end{array}$ & $4.2 \%$ \\
\hline Chatters & $\begin{array}{l}\text { A person who uses } \\
\text { the CoP mainly for } \\
\text { chatting with other } \\
\text { colleagues. }\end{array}$ & $\begin{array}{l}\text { Relatively high scores on } \\
\text { the active and passive } \\
\text { "chat" forms of interaction; } \\
\text { relatively low scores on the } \\
\text { other forms of interaction. }\end{array}$ & $3.1 \%$ \\
\hline Advisers & $\begin{array}{l}\text { A person who } \\
\text { informs (gives tips } \\
\text { to) others about } \\
\text { relevant content in } \\
\text { the CoP. }\end{array}$ & $\begin{array}{l}\text { Relatively high scores } \\
\text { on the active "send } \\
\text { recommendations" form of } \\
\text { interaction; relatively low } \\
\text { scores on the other forms } \\
\text { of interaction. }\end{array}$ & $0.5 \%$ \\
\hline $\begin{array}{l}\text { Community } \\
\text { leaders }\end{array}$ & $\begin{array}{l}\text { A person who is } \\
\text { actively involved in } \\
\text { all aspects of the } \\
\text { community. }\end{array}$ & $\begin{array}{l}\text { Relatively high scores on } \\
\text { at least the active forms } \\
\text { of interaction but also on } \\
\text { the more passive forms of } \\
\text { interaction. }\end{array}$ & $0.2 \%$ \\
\hline
\end{tabular}

The distribution of community member roles in each CoP is shown in Figure 9.10. The results reveal that most individuals in all CoPs use the CoP as a source of information, knowledge and experience. Just a small proportion of individuals within a CoP are community leaders, and these people stimulate others to participate.The distribution of the remaining types of members differs by community, but community $\mathrm{E}$ - the community focused on the care of elderly people-in particular shows some remarkable differences with the other communities. The CoP consists of a noticeably large number of posters and inactive members compared to the other CoPs. 
A more in-depth analysis of these specific cases shows that the number of inactive users is a consequence of the fact that many participants were not intrinsically motivated to participate in the CoP, and a substantial group of CoP members were automatically registered at the start of the CoP. This resulted in a large number of members but, consequently, a large number of inactive members as well. The number of posters in this community is also significantly higher than in the other CoPs. A more in-depth analysis of this member type shows that, especially in the first nine months, the number of posts in the CoP was remarkably high. The distribution of member roles in company $\mathrm{F}$ - the CoP that is focused on improving the production and distribution of food-shows a special number of appreciators and chatters. These two member roles are less visible within the community than the posters and commenters. For example, the chatters want to interact in a one-to-one mode with each other, while the appreciators want to acknowledge their appreciation for the meaningful contributions of others. In both company C (disability nursing) and company $\mathrm{G}$ (general social care), the number of commenters is remarkably high compared to the other member roles. This might indicate that the CoP consists of a diverse group of people who are willing to improve the knowledge in their domain by commenting on the input of others.

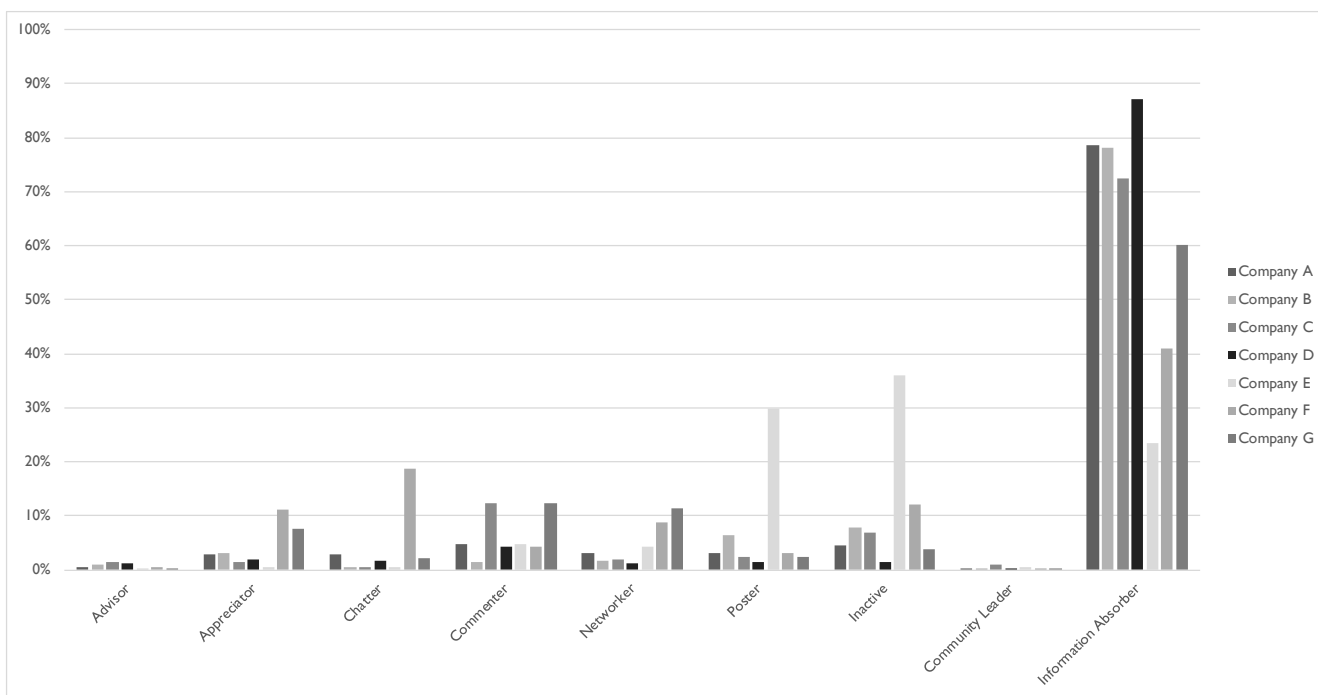

Figure 9. 10. Division of member roles within each CoP

\subsubsection{Influence of member roles in the development of CoPs}

The highs and lows visible in the Figures 9.3 to 9.9 show remarkable trends. To explain these trends, we checked for the most active member roles during that period. In three of the seven cases, the activities in the first few months of a CoP were mostly initiated by the community leaders. These individuals were motivated to stimulate the intensity of interactions and to improve the breadth and depth of the knowledge base. After a period, the number of interactions became increasingly dominated by the three most active types of community members: the posters, commenters and community leaders. This might be an indication of the increasing adoption of the CoP by other members and might also imply that the community leaders succeeded in their job. In the last few months, each CoP has reached its highest number of members, and the CoPs have become incorporated in daily practice, which means that individuals have come to know the extra features of the platform. Almost every community has 
followed the widely recognized phases from adoption to implementation to use; in each stage, both the intensity and variety of activities has risen. The other four cases develop themselves more gradually with no specific roles who are overrepresented in the development.

\subsection{Conclusions \& Discussion}

\subsection{Discussion}

The core of this research is to identify and analyse the development of CoPs from three perspectives. These perspectives are structured around three objectives: (I) identify and formalize the developments in the CoPs (2) identify community member roles in CoPs, and (3) analyse relationships between developments and the distribution of member roles. To realize the first objective, the scores of all social activities were transformed and analysed to ensure that each CoP could be compared. The second objective has been realized by assigning member roles to individual users based on a constellation of their social activities. To achieve the third objective, the results from the first objective were reflected upon along with the member roles originating from the second objective. The result is a methodology that solely uses available online data, data that is collected and stored within almost every online community software platform. This fact alone makes it a particularly suitable method for application at the community management level, and in the CMEM.

Community Development: In this study, we have a sample of seven CoPs which differ in purpose, progress and success. Based on the results, we see clear developments. Existing community phase models predominantly focus on 'ideal' community development based on successive phases (Iriberri \& Leroy, 2009). Nonetheless, analysing the starting phase in the graph purely based on all activities in all organisations, but also after closer zooming in on the different cases, shows that the developments in CoPs is characterized by small peaks and lows during the first five months: the implementation phase. After these five months, the community activity gradually increases each month. Two decent explanations for this are that in the first few months all community members participate out of curiosity or the CoP is initiated as a strategic CoP. The first implies a 'novelty fatigue', which represents the point at which individual excitement or curiosity about something new are beginning to disappear (Feenberg \& Bakardjieva, 2004). Declining visitor numbers and related social activity are the result. Second, in case of strategic decisions, the CoP is initiated by top management, and because enrolment is "strongly encouraged by top management", these members feel pressured to visit the CoP now and then (Van Yperen et al., 2016). A plausible explanation for the decrease in social activity after a few months is also given by Dubé, Bourhis, \& Jacob (2005), who recognize the environment, the relevance of the CoP's objectives to the professional's daily work, and the degree to which the CoP is embedded in the organisational structure of an organisation as the three most important structuring pre-conditions for successfully launching a CoP. Regardless, in these first few months, the core of the CoP is formed. Community members who see the importance of the CoP will continue to participate after these first months. Reflecting again on these developments with the community member life cycle initiated by Sonnenbichler $(2010)$ in mind, reminded us that most CoPs initially see significant development, after which a temporary dip in the growth occurs. This also largely corresponds with the findings of Rowe, Fernandez., Angeletou \& Alani (2012), who have shown that the progression in a CoP has its ups and downs, especially in the first few months of a CoP.After these months, reconsiderations in the implementation strategies and in the expectations seems to occur which results in a more sustainable progression. 
These findings are based on the normalized scores of all activities in the seven cases.An additional test has been conducted to evaluate the developments in the separate cases. Each case has been individually evaluated to determine which typical community development patterns emerge. The establishment of individual patterns differs greatly per case, but the development of these cases is often comparable. Two of the seven cases show typical community development patterns. These patterns are similar in the composition and normative intensity of the different types of social behaviour. However, the momentum that these patterns emerge differ per case. Four cases show a knowledge-centred pattern which implies that the focus and/or behaviour of the community members in the first 14 months of the CoP is on optimizing knowledge management within the organisation. A last typical pattern is the dialogue-centred pattern, this implies a more personal communication centred type of social behaviour in the CoP. It appears that these organisations and its employees are willing to reduce the social distance between the employees by encouraging dialogue between them. It is also striking that in the inter-organisational CoPs the normalized amount of personal conversations (chats) forms a considerable proportion of the total number of social interactions. This could imply that in this community form, the participants have a greater need to find relevant contacts beneficial for their work.

Member roles: Most community members use CoPs as a resource for information and knowledge. However, there is also a substantial number of community members who participate in a more active manner. For a variety of reasons, these individuals continuously ensure the further development of knowledge within the network. Wenger \& Snyder (2000) describe this core group of community members as the intellectual and social leaders, as their passion for their work and their desire to improve collaboration within their professional domain "energizes the community". This group - in this study defined as the community leaders - is the community's lifeline and is crucial for the success of the community. However, the group of posters and commenters also play a significant role in the success of the CoP, as they ensure the content production and thus the heart of the knowledge base due to their contributions in conversations (Fagnan, Rabbany, Takaffoli, Verbeek, \& Zaïane, 20I4). Other types of community members identified in this study are the networkers, appreciators, chatters, advisors, information absorbers and the inactives.

Influence of member roles in the development of CoPs: The dominant group in the first few months is usually reserved for the community leader. This often formally assigned (group of) individual(s) actively engage in the CoP to stimulate the social interactions between registered members. In most cases these individuals succeed in this activity; other members see the benefits of social interaction behaviour, adopt the CoP and incorporate it into their daily labour use and create threads or comments or express appreciation (Wenger, 2000). This active core group of a CoP also takes responsibility for the motivation and encouragement of the information absorbers by virtually and physically visiting groups of people to introduce to them the benefits and opportunities of the CoP and by demonstrating fewer active forms (e.g. appreciating or advising) of social interaction that still stimulate the development of the CoPs. This explanation is given by the formal community managers who were interviewed in Chapter 4. Given the increasing diversity in the types of social interaction, it is very likely that these community leaders are successful in their actions. 


\subsubsection{Conclusions}

Every CoP has a unique genesis. For example, some are introduced by their end users, while others are initiated at the management level. The central research question in this study was as follows: Which aspects of community development patterns characterize the development of a community of practice? In answering this question, web-based social interaction data are used to show the community development patterns that show community developments. By splitting the research question into three complementary objectives, we were able to formulate an adequate answer to this question. The three objectives were: $(I)$ to identify and formalize the developments in the CoPs (2) to identify community member roles in CoPs and (3) to analyse the relationships between developments and the distribution of member roles.

Community development: the development of most CoPs shows strong similarities with a symmetric triangle in an uptrend. This means that the developments are continuously subject to ups and downs. Most CoPs often start with an active period with several individuals, while the attention of many subsequently reduces; the CoP is not yet fully integrated in the work processes of these members. As soon as the active group of community members notice the diminished activity, new strategic choices are made, after which new activities are organised. The result of this is a new wave of activities, that encourages present members, as well as new members to participate (again). This moment is often a deciding one for the success of a CoP. If few people feel encouraged in this phase, many participants become demotivated. This development seems to occur in case $\mathrm{E}$, where the social activity within the CoP decreases.

Member roles: A CoP consists of a diverse group of community members who start all kinds of different types of social interaction. The majority consists of individuals who benefit from the knowledge and expertise of others. In relative terms, a much smaller number of the community members are actively involved in creating, sharing or appreciating content: they are the catalysts of social interactions and knowledge management. The relatively large number of information absorbers in the CoPs gives no reason to doubt a CoP's success. It is likely that the information absorbers gather knowledge and put this knowledge- consciously or unconsciously - into practice, which is also beneficial for the whole organisation.

Influence of member roles in the development of CoPs: An in-depth analysis of the relationship between the member roles and the stages in the CoP show that in three of the seven cases, a community leader actively engages in the process of activating community interactions. In the other four cases, the CoP has, from the beginning, had an engaging group of members. The dominant member roles in these four cases are: commenters, posters and chatters.

Existing research predominantly focus on CoPs from an ideal perspective, which defines successive steps towards developing a successful CoP.This research gives a realistic and factual picture of the development of CoPs. In this study, the focus was on two important topics in the development of CoPs: the development of social interactions and the types of members who participate in the CoPs. Developing the description of the community development patterns and defining the member roles and relationships between them enabled us to describe the development of CoPs and understand their development. For community leaders, information specialists and HR professionals responsible for the development of employees and their social capital, it is recommended that the insights in this chapter will be used for the systematic 
monitoring of their own CoPs. CoPs can develop in multiple preferred directions, by focusing on certain patterns, by formulating measurable objectives and by organizing activities to steer or control these patterns. Continuous monitoring of trends and developments in a CoP gives community management control over the different layers of the community. For example, when a community primarily consists of inactive members, it is worthwhile to start a discussion (onor offline) with these individuals to identify their needs and expectations. If the CoP mainly consists of posters, it might be a beneficial idea to help these posters writing posts that start discussion. In the end, the quality of the community will always improve if there are grounds for interaction and discussion.

\subsubsection{Limitations}

The exploratory nature of this research automatically leads to a few choices that are open to discussion. First limitation in this study is the limited context assigned to the use of web-based social interaction data. The quantity gives a reliable picture of the community developments but does not provide insight into the reasons behind these developments. For future research, it is recommended that the web-based social interaction data in this study are combined with content analysis in the CoP to explain the reasoning behind certain developments.

Second, the purpose of this explorative research was to provide insight into developments within and from CoPs, using the web-based social interaction data from seven cases that use the same online community software. We made use of seven cases with in total 35.299 social interactions divided over five types of social interactions. To be able to generalize, this type of data-driven needs and to avoid the impact of outliers, it is preferable to have access to a substantial number of cases and at least for instance 500.000 social interactions. The more data we can collect from different cases, the smaller the uncertainty about certain developments (Bishop, 2013).

A final limitation in this study is the lack of insight into the order of responses. Based on the web-based social interaction data it is not possible to trace when a reaction is based on another reaction or on the general post. Insight into these data would provide an even better picture of the processes that influence the community development. It is therefore recommended to create a situation in follow-up research where threads and conversations can be made transparent. 



\section{0}

\section{Individual Network Positions in Communities of Practice}

\section{I. Introduction}

In the previous chapters, the CoP has been studied from multiple perspectives varying from determining the factors that affect individual community behaviour to defining how communities develop over time. In this chapter we conduct an explorative study to identify members' social network positions in CoPs and the relative distribution of these positions in a CoP.

Ideally, CoPs emerge spontaneously without any top-down interventions. The reality is that the creation of a CoP is often part of a systematic and strategic approach led by an organisation to promote the effective management of intellectual and social capital (Lesser and Storck, 200 I). To ensure that organisations fully benefit from the advantages of CoPs, it is crucial to adopt an appropriate knowledge management system (KMS), including a knowledge management strategy, knowledge processes and knowledge management tools (Canzano, 2004), that pays special attention to the continuing professional development of the community members (Macia \& García, 2016). Setting up a good KMS means - among other things - that the right people are put in the right positions within the CoP (Bourhis, Dubé, \& Jacob, 2005). By assigning formal roles, the first step is to gain control over the developments in the CoP. However, community members also take on informal social positions. Some members are born connectors, while others are typical contact points or knowledge distributors. According to Zhang \& Watts (20I5), community members regularly gain new identities during the process and throughout duration of the CoP; this results in the identification of competent and incompetent members, transformations from newcomers to experts, and other users' loss of interest - temporarily or permanently - in the developments within the CoP. Identifying these positions, the changes within these positions, and the roles these people have helps organisations to give members tailored support and provides insight into the social capital that someone offers or develops (Chang \& Chuang, 20I I; Kovanovic, Joksimovic, Gašević, \& Hatala, 20I4).

Thanks to the Internet and its relational data storage, it has become much easier to quickly identify and pinpoint the social position of an individual by using social network analysis techniques. The social network perspective has proven to be an effective and efficient enabler for the identification of various social network positions (Kratzer et al., 2016). Identifying these positions is a first step, and the second step is to identify those factors that relate to the composition of these social network positions. The research question that accompanies this chapter is as follows: Which factors affect the formation of social network positions in CoPs?

\subsection{Theory}

\subsection{Data Revolution}

Big (social) data is playing an increasingly large role in current academic research. As stated by Mayer-Schonberger \& Cukier (2013), all aspects of life are now transformed into quantifiable 
data. Additionally, research in the field of online CoPs has an ever-expanding role reserved for 'new', bigger, data-based analysis. Traditional qualitative and quantitative research methods can be complemented by large datasets that are more objective (Schäfer \& Es, 2017). Datasets that can be used to understand the complex and dynamic reality of current society. Undeniably, these social data involve a process of abstraction that forces researchers to make certain compromises as the data are generated, collected, selected and analysed (Elmer, Langlois, \& Redden, 2015), but every step in this process is a step towards a methodology that enables us to test theoretical models faster and possibly even more objectively. Faraj et al. (2016) pose that existing research on online communities has mainly focused on why people joint these communities and less on the activities of these individuals in these communities. The online community software that aims to help the development of CoPs generates large amounts of valuable social interaction data-data that show who speaks to whom, when they speak, how often they speak, et cetera. Plenty of options to improve our understanding of the activities. These data offer new perspectives on developments in CoPs; one of these developments is pinpointing the position of individual community members based on their social interactions in the CoP.

\subsubsection{CoP network positions}

The individual social network position is often used as a valid indicator for the social capital that someone offers (Kovanovic et al., 2014) and the social capital that someone develops (Chang \& Chuang, 20II). Following the social capital literature, a community member's social network position is reflected by his or her relationships with others in a social system. The actor who holds a more central position often possesses more direct social relationships with others in the social system. The individual's $s$ o c i a network position is an important source of group identity, social support, and friendship (Hsiao \& Chiou, 20I2).

Speaking about member types and social network

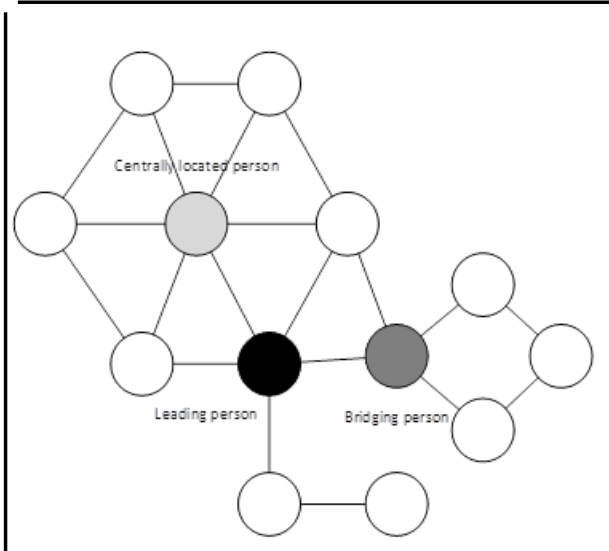

Figure 10.I. CoP network positions positions presupposes that an individual's identity is fundamentally temporal. Wenger (1999) mentions that membership in a CoP is a temporary event, it changes throughout the course of the community membership from for example, a peripheral position to an insider position or a boundary position. To obtain a good indication of the position of individual community members, it is worthwhile to measure the node centrality of individuals. Previous studies have often used these centrality measures to determine whether an individual has a leadership position; the more central the individual is in a network, the more leading his or her position (Johnson et al., 20I5). However, objectively looking at network centrality could imply that it can be interpreted from more perspectives than only a leadership perspective.

The position of an individual in a social network is often measured by using one of the centrality measures introduced by Freeman (1978). The term centrality refers to the relative central location of given units within a structure. In his earliest work Freeman (1978) conceptualized centrality as patterns of communication where different individuals functioned as hubs of a 
wheel or as the point at the centre of a star. In the following three paragraphs, the three dominant network centrality measures are prominently featured: degree centrality (a centrally located person), betweenness centrality (a bridging person), and closeness centrality (a leading person). Figure 10.I shows a visual representation of the three primary network positions in this study.

\section{Centrally located person}

If researchers define centrality, it is often based on degree centrality. Cho, Gay, Davidson, \& Ingraffea $(2007$, p6) define centrality as "the degree to which an actor is connected to others in a given social network". This definition highly corresponds with the description used by Wellman \& Berkowitz (1988), where centrality signifies two nodes that are directly reachable from one another and are thus only one path-distance away. It is assumed that network members with a high degree centrality must have a more active, powerful, respected, and visible location in the network than the more peripheral members. One of the main arguments is that these network members have many ties and connections to other members in the network or graph (Freeman, 1978). Community members with a high degree centrality are often called hubs or central connectors. With a high degree of centrality, these individuals have the most influence in a network since they have many direct connections. The central position of these individuals often makes an organisation quite vulnerable if these people leave the organisation.

\section{Bridging person}

Another centrality measure introduced by Freeman (1978) is the betweenness centrality. This measure aims to calculate along how many paths an individual network member is the bridging person, which results in an estimation of the extent to which that person can disrupt or distort system-wide communications but also stimulate social interaction. For given network member A, betweenness centrality is mathematically calculated as the number of shortest paths between any two other network members that "pass through" network member A (Freeman, 1978). Betweenness centrality is a meaningful measure because it takes into account both the local connections of a network member and the member's global position in the network (Johnson et al., 20I5). Persons with high betweenness are often positioned in the collaborative and communication network between people who are not directly connected. Therefore, these network members are often seen as brokers, bridges, lead users or boundary spanners (Kratzer et al., 2016). These individuals position themselves between different clusters in a network and are critical to gathering and transferring information.

\section{Leading person}

Closeness centrality is a third centrality measure that tests the degree to which an individual is close to all other members in a network. This measure can be conceptualized as the position where a person has "easy access to important information without having a great number of contacts" (Brass \& Burkhardt, 1993, p.446). The peripheral location of a network member is defined as the sum of its distances to all other nodes. It gives an indication of how long it will take to sequentially disseminate a message to all other members in the network. It is assumed that people with a high closeness centrality have or take a more leading role in a network (Sonnenbichler, 20l0), since these individuals have the opportunity to reach a large group of people.According to Freeman (1978), the closeness of a focal person can be conceptualized as independence and therefore represents a measure of independent access to others without interference from others. 


\subsubsection{Influencing factors}

What factors affect the formation of social network positions? That is the question in this chapter.The relationship between motivations, opportunities, abilities and communality (MOAC model) and individual social network positions will be examined. The MOAC model builds on the work done by psychologists to determine the factors influencing an individual's behaviour. The original MOA model (Maclnnis \& Jaworski, 1989) is already a well-established theoretical base for understanding work performance (Siemsen et al., 2008). Academic researchers using the influencing factors in the MOA model interpret the first factor 'motivation' differently. Some construe motivation as a driving force for behaviour by measuring the level of motivation (Kettinger et al., 20I5), while others take the perspective in which it is an act or process of motivating (Siemsen et al., 2008). In this research, motivation in the MOAC model is approached from the perspective of the driving factors that influence or explain individual behaviour. The second element in the MOA model is opportunity, opportunity reflects the presence of enabling environmental mechanisms that facilitate task performance (Fadel \& Durcikova, 20I4). The third element is ability which is defined as the internal capability related to an action (Siemsen et al., 2008). This can be the ability to spread information, influence others or obtain and apply relevant information (Cross \& Cummings, 2003). The last element added to the MOA model is communality. Participating in and feeling attached to a group is a central aspect of communality also defined as a sense of community (Abraham,Ajith, 2010). The group attachment of members to a CoP might be a good indicator for the level of social behaviour of an individual (Hrastinski, 2009), since people who have a stronger feeling of alignment with the norms and values in a community are more willing to actively take part in it. In this study, the extensive MOAC model in its original form is used in relation to individual network positions within a CoP.Which means that the motivation, opportunity, ability and communality are used as latent variables instead of the underlying variables as outlined and tested in chapters 6 to 8 . In the coming paragraphs, the four elements in the MOAC model will be described in more detail.

An individual's motivation

Motivation is often defined as an individual or unit's willingness to act (Rothschild, 1999; Siemsen et al., 2008), and motives are often considered an important building block for motivation in forming an attitude towards community behaviour (Chen, Yang, \& Tang, 20I3). Strong motives for showing behaviour are a good indicator for the motives people have but also provide insight into the 'why' question of a behaviour. Professionals who are highly committed to the purposes of a community are more willing to actively work in the CoP (Liu, Wagner, \& Chen, 20I4). Individual commitment stems from their motivation to perform in a certain manner. The more diverse their types of motives are, the more positive their perception regarding the CoP will be (Chen et al., 2013). It is likely that people with a high diversity of motives to participate also have a more intense motivation to do so. A considerable number of motives lie at the basis of the decision to participate in a CoP.Wasko \& Faraj (2005) conducted a study to examine the motives of community members to share their knowledge. To understand this, theories of collective action were applied to examine how individual motives and social capital influence knowledge contribution in electronic networks. Their study resulted in a number of diverse motives for knowledge exchange; these are primarily intrinsic, such as the willingness to improve one's reputation, social network position and enjoyment. Ardichvili et al. (2003) conducted a comparable study and showed that several motives affect knowledge exchange, and these motives can be divided into two categories: organisation-based considerations and self-based considerations. A supporting organisational culture belongs to the category of organisation-based considerations, and reputation and career stage belong to the category 
of self-based considerations. Particularly in the context of group behaviour, as discussed in Chapter 3, some motives are important primarily because they are related to knowledge exchange, knowledge acquisition and social interaction: informing (Hara. \& Hew, 2007; Lee \& Ma, 20I2), work performance (Bock et al., 2005; Han et al., 2007; Kulkarni et al., 2007), social reputation (Bettiga et al., 2018; Kulkarni et al., 2007) and subjective norms operationalized as social pressure (Lewis, Agarwal, \& Sambamurthy, 2003; Scarbrough, 2003). In this part of the dissertation, the motivation has been constructed based on the intensity with which certain motives apply.

HIa: The more intense an individual's motivation, the higher the centrality-located position of that community member.

HIb: The more intense an individual's motivation, the higher the leading position of that community member.

HIc: The more intense an individual's motivation, the higher the bridging position of that community member.

HId: The more central the position of a community member, the higher the individual's motivation to participate.

Hle: The higher the leading position of a community member is, the higher the individual's motivation to participate.

HIf: The more bridging the position of a community member, the higher the individual's motivation to participate.

\section{An individual's opportunity}

An individual's motivation describes the mental state of the community member.An individual's reported opportunity refers to the conditions supported by the organisation in which the community member participates (Hung, Sirakaya-Turk, \& Ingram, 20l I; Turner \& Pennington, 2015). Opportunity is the context in which, among other actions, knowledge exchange, collaboration and social networking can occur. Opportunity is positively associated with knowledge sharing, which indicates that people who have more opportunities have a greater chance of achieving a more significant position within the CoP (Turner \& Pennington, 20I5). Ibarra \& Andrews (1993) suggest that position in a network affects the positive perceptions people have of their organisations, which implies that these people have a more coloured view of the opportunities they have to participate in a CoP. Therefore, there is a significant chance that a person's position in a network is affected by his or her perception of the opportunities he or she has to act in a meaningful manner. One of the factors identified often as a barrier to social interaction behaviour in CoPs is the lack of time for such activities due to the stressful daily professional situations in which they should be embedded (Correia et al., 2009; Orlikowski, 1993). In addition, the flexible way of working that has been introduced in many organisations has entrenched employees' desire for more freedom to organize and plan their work activities; these employees want more work autonomy (Cardona, Lawrence \& Bentler, 2003; Salanova, Peiró, \& Schaufeli, 2002). In the end, the online component of CoPs requires fertile grounds for employees to participate in CoPs. The online support systems must contribute to the efficient and effective social behaviour of the community members. Bhattacherjee (200I), Kettinger et al. (20I5) and Venkatesh, Morris, Davis \& Davis (2003) define this fertile climate as excellent facilitating conditions; people need practical, barrier-free and secure access to online systems with special attention for support and training.

The previous research described in chapter 7 has shown that the factors of time, work autonomy and facilitating conditions are latent variables for the construct of opportunity. In this 
exploratory study, it is assumed that these variables can be combined under the construct of opportunity, which affects the various social network positions. People with an increased sense of reported opportunity are more inclined to take up certain relevant social network positions.

H2a: The higher an individual's reported opportunity, the higher the centrality of the position of the community member.

H2b: The higher an individual's reported opportunity, the higher the leading position of the community member.

H2c: The higher an individual's reported opportunity, the higher the bridging position of the community member.

H2d: The more central the position of a community member, the higher the perception that community behaviour-stimulating opportunities are offered.

H2e: The higher the leading position of a community member, the higher the perception that community behaviour-stimulating opportunities are offered.

H2f: The more bridging a community member's position, the higher the perception that community behaviour-stimulating opportunities are offered.

An individual's ability (self-efficacy)

Communication media - the foundation of online-based CoPs-have enhanced the individual's capacity to find information pertinent to the performance of daily tasks (Malone, 2002). Despite the benefits of these communication media, these media also require digital skills to meaningfully use these media (Tseng \& Kuo, 20I4). However, ability-or self-efficacy (Bandura, 1977) — can be defined more broadly than only digital skills. These new communication media also require new forms of collaboration and social interaction (Cadiz et al., 2009). Thus, ability is also the extent to which an individual possesses the necessary skills and abilities required to commit an action (Macinnis et al., 199I). Wasko \& Faraj (2005) have found evidence that individuals with higher levels of expertise are more likely to provide useful advice and share relevant knowledge than people who feel that their expertise is inadequate. Therefore, the selfconfidence of an individual regarding the skills he or she has is an important indicator of the actual behaviour that he or she will exhibit in the CoP and thus for the position that he or she will consciously or unconsciously take in the CoP.These skills are operationalized in three fields: an individual's ability to express him or herself in a meaningful manner (Siemsen et al., 2008; Turner \& Pennington, 2015), the ability to contact and consult peers and acquaintances (Cadiz et al., 2009), and the digital skills to use digital communal spaces (Tseng \& Kuo, 20I4; Venkatesh et al., 2003). The existing research on social network positions has primarily focused on individual ability as an outcome of a social network position. People with a central social network position are often associated with good performance and promotion in their organisation (Brass, 1984; Cross \& Cummings, 2003). However, it is assumed that this also applies in the inverses: an individual's social network position is affected by the individual's ability to participate in a CoP in a meaningful manner, in this research operationalized as perceived ability or self-efficacy. The hypotheses defined to analyse the role of an individual's ability in relation to his or her network position are as follows:

H3a: The higher an individual's perceived ability, the higher the centrality of the position of the community member.

H3b: The higher an individual's perceived ability, the higher the leading position of the community member.

H3c: The higher an individual's perceived ability, the higher the bridging position of the community member. 
H3d: The more central the position of a community member, the higher the perception of the individual's ability.

H3e: The higher the leading position of a community member, the higher the perception of the individual's ability.

H3f: The more a community member has a bridging position, the higher the perception of the individual's ability.

\section{An individual's sense of community (communality)}

Creating, improving and using social capital in an organisation is one of the ultimate ambitions of organisations. The social capital theory primarily concerns the formation and maintenance of social relations that help individuals acquire the power to obtain available resources through their identity as members of the social network that forms the foundation of each CoP (Tseng \& Kuo, 20l4). Maximizing the use of social capital helps organisations become more efficient, effective, innovative and sustainable compared to other organisations.

To realize this, it is important for organisations to create an atmosphere that supports the development and improvement of social capital. Creating a sense of community is then one of the first tasks of the community manager, as this often stimulates the social interactions between individual community members. The sense of community is often seen as an incentive for both individual and collective community behaviour. By investing in social capital, which stimulates social behaviour, an individual may gain personal rewards while at the same time contributing to the creation of social relations as a positive network externality (Adler \& Kwon, 2002; Heinz \& Rice, 2009). Such an investment in developing social capital can produce additional network externalities aside from social relations, such as connectivity and communality (Nahapiet \& Ghoshal, 1998).

To create an effective community, it is, according to Kruse \& Louis (1993), crucial to have shared values and norms of behaviour: "While Western philosophy has often emphasized the importance of individual moral development and responsibility, the communitarian perspective focuses on the balance between the individual and the group. The development of strong, ethically based and morally binding norms of behaviour are one characteristic of an effective community" (Kruse \& Louis, 1993, p.5)It is assumed that when focusing on a communitarian perspective, the sense of community and ultimately the community commitment of individuals will increase. Dawson (2008) found - in an educational context - evidence that students' sense of community membership was positively related to their closeness and degree centrality. In this research, the term communality is used as an umbrella term for the perception of a communality feeling in CoPs. The discussions around social network positions have resulted in the following hypotheses:

H4a: The higher an individual's perceived communality perception, the higher the centrality of the position of a community member.

H4b: The higher an individual's perceived communality, the higher the leading position of a community member.

H4c: The higher an individual's perceived communality, the higher the bridging position of a community member.

H4d: The more central a community member is in a CoP; the higher the individual's perceived communality will be.

H4e: The higher the leading position of a community member, the higher the individual's perceived communality. 
H4f: The more bridging a community member's position, the higher the individual's perceived communality.

\subsection{Method}

The method used as part of the community monitoring and evaluation methodology (CMEM) contributes to our understanding of the importance of network positions in CoPs. In this study, the focus is on descriptive and diagnostic community analytics (Banerjee et al., 20I3). These analytics are operationalized in social network positions determined by using social network analysis (SNA) techniques. The endogenous network effects for example represented as social network position play an important role in tie formation dynamics. In general, these network effects are often estimated based on the statistics derived from the network and computed by counting, for example, local network configurations in the observed network (Wang \& Robins, 2009).A more in-depth description of the social network position is defined in 10.3.2. The datadriven process introduced in Chapter 5 has been followed to ensure that the data is valid and prepared for analysis and interpretation. More specifically, this means that the data is prepared for the network analyses. In this study, the same four cases form the core as in chapters 6 to 8 . These four cases all meet the definition of a CoP as used in this dissertation: a social network where people in an organisational context come together around a common topic, passion or interest and regularly interact on- and offline with a focus on knowledge management, innovation, learning and social networking.

\subsection{Stimulating factors}

In Chapter 3, the motivation, opportunity, ability, and communality model were introduced as a model for understanding social behaviour in CoPs. In Chapters 6, 7 and 8, the individual constructs were tested based on the area to which they each apply. Motivation and ability were therefore assigned to the individual level, opportunity to the organisational level, and communality to the collective level.

The underlying factors (e.g., subjective norms, social reputation, facilitating conditions) under the motivation, opportunity, ability and communality model (MOAC model) defined in Chapter 3 were tested with a survey in Chapters 6,7 and 8. With that approach, it became possible to explain individuals' social behaviours in CoPs. However, for this chapter, the focus is on the parent variables - motivation, opportunity, ability, and communality-in explaining the network positions of individuals in the community network. For the sake of parsimony and clarity, the underlying factors in four independent variables have been merged in order to examine the relationships with the different network positions. These four independent variables are motivation, opportunity, ability and communality. The results of the survey are combined with web-based social interaction data in this chapter. By giving their full name and email address, respondents enabled us to merge the web-based social interaction data with the survey data. After merging the datasets, all traceable data were deleted to ensure anonymity and guarantee that ethical standards were respected. The final document has only unique identifiers without further traceable background information for all participating community members. The four factors will be used to empirically test the influence of the factors on individual social network positions in the four CoPs participating in this study. Only those respondents who gave approval to merge their answers in the questionnaire with their behaviour in the CoP are analysed.This resulted in a rather smaller sample of 144 respondents. 


\subsubsection{Social network positions}

Insight into community member roles: a network perspective

The four CoPs participating in this study all use the same online community software. The digital traces that the participants in the CoP leave are registered and stored and are after processing used in this study to analyse for network patterns.

These data consist of the social interactions between individuals within their shared CoP. It is possible for each member in the CoP to start five different types of social interaction with a colleague (Table I0.I). Converting these actions by means of the social networking analysis and visualization tool Gephi (Bastian, Heymann, \& Jacomy, 2009) into network graphs for each individual CoP results in a one-mode network graph with different types of social relationships. This network graph will be studied by means of the three centrality measures: degree centrality, closeness centrality and betweenness centrality (Table 10.2). Normalized scores from the last two centrality metrics are used to ensure that the data is less skewed. Community members who have not entered in any type of social interaction are scored with a ' 0 ' on each form of centrality.

Table 10.I

Types of Social Interaction

\section{Type of social interaction Implication}

Comment

Recommendation

Appreciation

Following

Chatting

\section{Person A is commenting the post of Person B}

Person $\mathrm{A}$ is recommending a post to Person $\mathrm{B}$

Person A appreciates the contribution of Person B

Person A follows the contributions of Person B

Degree centrality is a simple way to gauge the prestige of a node; the more connections a node has the higher its degree centrality. In the formula, i represents an individual community member, $j$ represents all other nodes, $N$ is the total number of nodes, $x$ is the adjacency matrix in which cell $x i j$ is defined as $I$ if node $i$ is connected to node $j$, and 0 if this is not the case. Degree centrality is often the first value people look at in social networks to gauge importance. In the context of this study, this could mean that individuals with a high degree centrality are regularly consulted by peers or proactively interact with others. Closeness centrality measures how many steps it takes on average for an individual node to reach every other node in the network (Abraham, Ajith, 20I0). In formula $d,(y, x)$ represents the distance between vertices $x$ and $y$. Closeness centrality often refers to a normalized form that represents the average length of the shortest paths instead of their sum. Finally, betweenness centrality measures the extent to which an actor has a bridging position between two other actors (Kratzer et al., 2016). The formula for betweenness centrality shows $\sigma_{\text {st }}$, which stands for the total number of shortest paths from node $s$ to node $t$, and $\sigma_{\text {st }}(v)$ stands for the number of those paths that pass through v. 
Table 10.2

Formulas for Centrality Measures

$\begin{array}{lll}k(i)=\sum \frac{n}{i} x_{i j} & C(x)=\frac{1}{\Sigma_{y} d(y, x)} & g(v)=\sum_{s \neq v \neq t} \frac{Q_{s t}(v)}{Q_{s t}} \\ \text { Degree Centrality } & \text { Closeness Centrality } & \text { Betweenness Centrality }\end{array}$

Insight into social behaviour stimulating factors

The four constructs of motivation, opportunity, ability and communality (MOAC) are operationalized in latent variables and were tested by conducting an extensive survey with the community members in four organisations. Motivation is measured via four latent variables that are proven to be stimulating factors for acting in a certain manner: the aim to stay informed, the aim to improve work performance, the motive to ensue or maintain a social reputation, and the social pressure from peers and acquaintances to participate. Opportunity is measured with three latent variables: available time to act, level of work autonomy, and facilitating conditions that stimulate individual social behaviour. Ability is measured with the following variables: the perceived ability to express oneself in a communal environment, social networking skills with others for consultation or social interaction (perceived ability to contact), and perceived digital skills to use the increasingly online technologies that are fundamental to current CoPs. The last construct, communality, is operationalized in an individual's perception of a shared language, the extent to which a sense of shared identity is present in the CoP, and the perceived form of shared leadership.

A significant number of community members $(n=144)$ completed the survey and gave approval to connect their answers to their web-based social interaction data. We used a 7-point Likertscale for each of the items. The scale ratings run from I = strongly disagree to $7=$ strongly agree. The first part of this study focused on calculating the centrality measures for each community member during his or her entire period of social behaviour in the community, purely based on the web-based social interaction data and test by means of PLS-SEM (software package: SmartPLS) which stimulating factors affect these network positions. The community is, in that case, seen as a large network of community members interacting with each other. In the second part of this study a comparable method has been applied, with the difference that the community is split into smaller units of analysis to identify whether the social network positions and the variables that explain the emergence of these social network positions change compared to the full dataset (models 3 through 6). The decision to split the social activities is made based on the maturity of the CoP and the number of social activities in the CoP, which also varied in each case.

\subsection{Results}

\subsection{Explaining the individual social network positions}

Merging survey data with the social network positions in a CoP provides us with insight into potential explanatory variables that give an indication of the position of individuals in their network. The position of individuals has been tested by using centrality measures: degree centrality, closeness centrality and betweenness centrality. These measures give an indication of the position of an individual compared to others. A comprehensive survey among I44 respondents shows the indicators that affect an individual's social behaviour in a CoP. The duration of each CoP has been split into two phases: the first 9 months of a CoP and the second 
9 months of the CoP.The decision for this split is based on the duration of the shortest running CoP. The shortest running CoP is 18 months. In order to obtain comparable data sets, the same durations were chosen. This resulted in 97 respondents for phase I (models 2 and 5) and 126 respondents for phase 2 (models 3 and 6). In total 6 research models were analysed: the relationship between MOAC and the social network positions and vice versa with the complete dataset of the CoPs and for the two phases.As indicated in 10.3.2.2., models were analysed using SmartPLS, a PLS structural equation modelling tool that assesses the psychometric properties of the measurement model and estimates the parameters of the structural model.

\subsubsection{Measurement model}

In the first step of creating the measurement model all specific items for the motivation, opportunity, ability and communality were added to the measurement model. The resulting scores in the cross loadings have made us decide to remove all items with scores lower than the threshold of 0.4 set by Hulland (1999). The result is that five low scoring items in the motivation construct are removed, two items in the opportunity construct, and two items in the communality construct.After this step, the indicator reliability was again tested by means of cross-loadings (Chin, 1998), which validates that each item in the resulting model loads higher on the assigned construct than on other constructs. Following the standard set by (Hulland, 1999; Kwong-Kay Wong, 2013) leads to the composition of the constructs (Appendix I0.I) based on cross-loadings higher than 0.4 (Appendix 10.2). This standard applies to exploratory research in which this research is carried out. However, all loadings score between the acceptable 0.6 and 0.99 .

In the next step, the discriminant validity has been calculated. The calculation and evaluation of the discriminant validity of the constructs has been done by following the threshold set by Fornell \& Larcker (198I). These authors suggest that the square root of the average variance extracted (AVE) for each latent variable must be larger than the correlations among the other latent variables. This is the case for all models in this study, where the overarching model (Model I) shown in Table 10.3 is an important indicator. The composite reliability of the models has been measured to indicate their internal consistency reliability. The scores of the composite reliability of the different measures range from 0.68 to 0.92 , which exceeds the recommended threshold value of 0.6 (Bagozzi \& Yi, 1988). The threshold for the average variances extracted (AVE) is with scores higher than 0.5 , which is also higher than the threshold for the convergent validity (Bagozzi \& Yi, 1988), this confirms the convergent validity. The results are shown in Appendix 10.3.

Table 10.3

The discriminant validity of the measurement model

\begin{tabular}{llllllll}
\hline MOT & OPP & AB & COM & BRI & CP & LEA \\
\hline
\end{tabular}

Motivation $\quad 0.791$

(MOT)

Opportunity $\quad 0.415 \quad 0.802$

(OPP)

$\begin{array}{llll}\text { Ability }(A B) & 0.342 & 0.70 \mathrm{I} & 0.775\end{array}$ 


\begin{tabular}{lccccccc}
\hline & MOT & OPP & AB & COM & BRI & CP & LEA \\
\hline $\begin{array}{l}\text { Communality } \\
\text { (COM) }\end{array}$ & 0.352 & 0.475 & 0.468 & 0.828 & & & \\
Bridge (BRI) & 0.216 & 0.216 & 0.202 & 0.180 & 1.000 & & \\
$\begin{array}{l}\text { Central } \\
\text { person (CP) }\end{array}$ & 0.436 & 0.477 & 0.395 & 0.322 & 0.638 & 1.000 & \\
Leader (LEA) & 0.193 & 0.393 & 0.413 & 0.348 & 0.293 & 0.416 & 1.000 \\
\hline
\end{tabular}

\subsubsection{Structural model}

Table I0.4 and Table I0.5 represent the six structural models. In this results section, we report only the significant relationships between the factors. In the full network, a positive significant relationship has been found between motivation $(B=0.27, p<0.0 \mathrm{I})$ and degree centrality and between the opportunities one gets $(B=0.29, p<0.0 \mathrm{I})$ and degree centrality. There is also a positive relationship between the motivation of an individual and closeness centrality $(B=0.14$, $p<0.05)$, between the perception of ability and the betweenness centrality $(B=0.23, p<0.05)$, and between communality and the betweenness centrality $(B=0.17, p<0.05)$.

The second model - which reflects the first phase of a CoP, shows a positive significant relationship between an individual's motivation $(B=0.15, p<0.05)$ and degree centrality, between the opportunities one gets $(B=0.17, p<0.05)$ and the degree centrality and between the communality and the degree centrality $(B=0.1 \mathrm{I}, \mathrm{p}<0.05)$. There is also a positive path coefficient between an individual's perceived ability and closeness centrality $(B=0.224, p<$ 0.05).

In the third model, which is a representation of the second phase, the beta values of the individual's motivation $(B=0.16, p<0.05)$ and individual's opportunities $(B=0.22, p<0.05)$, and communality $(B=0.12, p<0.05)$ are positively related to the score on degree centrality. Betweenness centrality is positively affected by an individual's motivation $(B=0.1 \mathrm{I}, \mathrm{p}<0.05)$. 


\begin{tabular}{|c|c|c|c|c|c|c|c|c|c|c|}
\hline & & \multicolumn{3}{|c|}{ Model I:All phases } & \multicolumn{3}{|c|}{ Model 2: first phase } & \multicolumn{3}{|c|}{ Model 3: second phase } \\
\hline & & 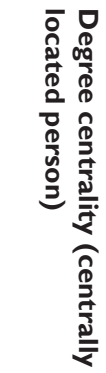 & 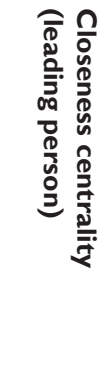 & 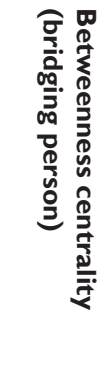 & 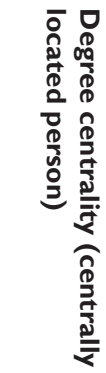 & 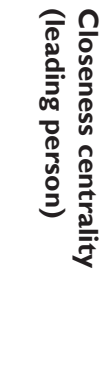 & 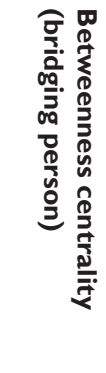 & 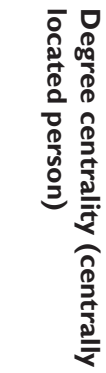 & 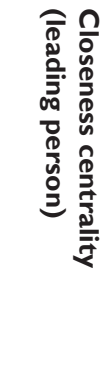 & 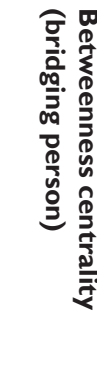 \\
\hline \multirow{4}{*}{$\begin{array}{l}\text { t- } \\
\text { score }\end{array}$} & Motivation & $0.272 * *$ & $0.137 *$ & -0.009 & $0.145^{*}$ & 0.086 & 0.101 & $0.160 *$ & 0.052 & $0.107^{*}$ \\
\hline & Opportunity & $0.285^{* *}$ & 0.082 & 0.156 & $0.172 *$ & 0.040 & 0.115 & $0.223^{*}$ & 0.202 & 0.067 \\
\hline & Ability & 0.076 & 0.069 & $0.227^{*}$ & 0.038 & $0.224 *$ & 0.067 & 0.078 & 0.191 & 0.102 \\
\hline & Communality & 0.076 & 0.061 & $0.170 *$ & $0.112 *$ & 0.110 & 0.069 & $0.120 *$ & 0.120 & 0.098 \\
\hline R-Square & & 0.212 & 0.302 & 0.072 & 0.212 & 0.129 & 0.072 & 0.202 & 0.206 & 0.080 \\
\hline
\end{tabular}

The fourth model is again a representation of a network overview based on all social activities in the CoPs. However, this model differs from the first model since in this model and in model 5 and 6 relationships are tested between the social network position measures and the factors from the MOAC model. This approach provides insight into the impact that network positions have on the various explanatory variables. Insights that contribute to an improved picture of the mutual differences between the network positions. In model 4, a positive significant relationship is found between degree centrality and the motivation $(B=0.50, p<0.01)$. Degree centrality $(B$ $=0.49, \mathrm{p}<0.0 \mathrm{I})$, closeness centrality $(B=0.24, \mathrm{p}<0.05)$ and betweenness centrality $(B=-0.17$, $P<0.05)$ are also positively connected to the individual's opportunity. A positive relationship has also been found between degree centrality $(B=0.33, p<0.0 \mathrm{I})$ and closeness centrality $(B$ $=0.3 \mathrm{I}, \mathrm{p}<0.0 \mathrm{I})$ and an individual's perceived ability to perform. Degree centrality $(B=0.29$, $p<0.05)$ and closeness centrality $(B=0.26, p<0.05)$ positively relates to the perception of communality.

The fifth model, which addresses the first phase in the CoPs, shows a positive significant relationship between degree centrality $(B=0.70, p<0.05)$ and individual's opportunities and with the communality $(B=0.63, p<0.05)$. Further, a significant positively relationship has been found between the closeness centrality and one's perceived ability to perform $(B=0.29, p<$ 0.05).

In the sixth model, which represents the second phase of the CoPs, positive relationships are found between degree centrality and motivation $(B=0.57, p<0.05)$, opportunity $(B=0.55, p$ $<0.05)$, perceived ability $(B=0.27, p<0.05)$, and communality $(B=0.31, p<0.05)$. A positive significant relationship has also been found between closeness centrality and opportunity $(B=$ $0.25, p<0.05)$ and perceived ability $(B=0.30, p<0.05)$. Finally, a negative significant relationship 


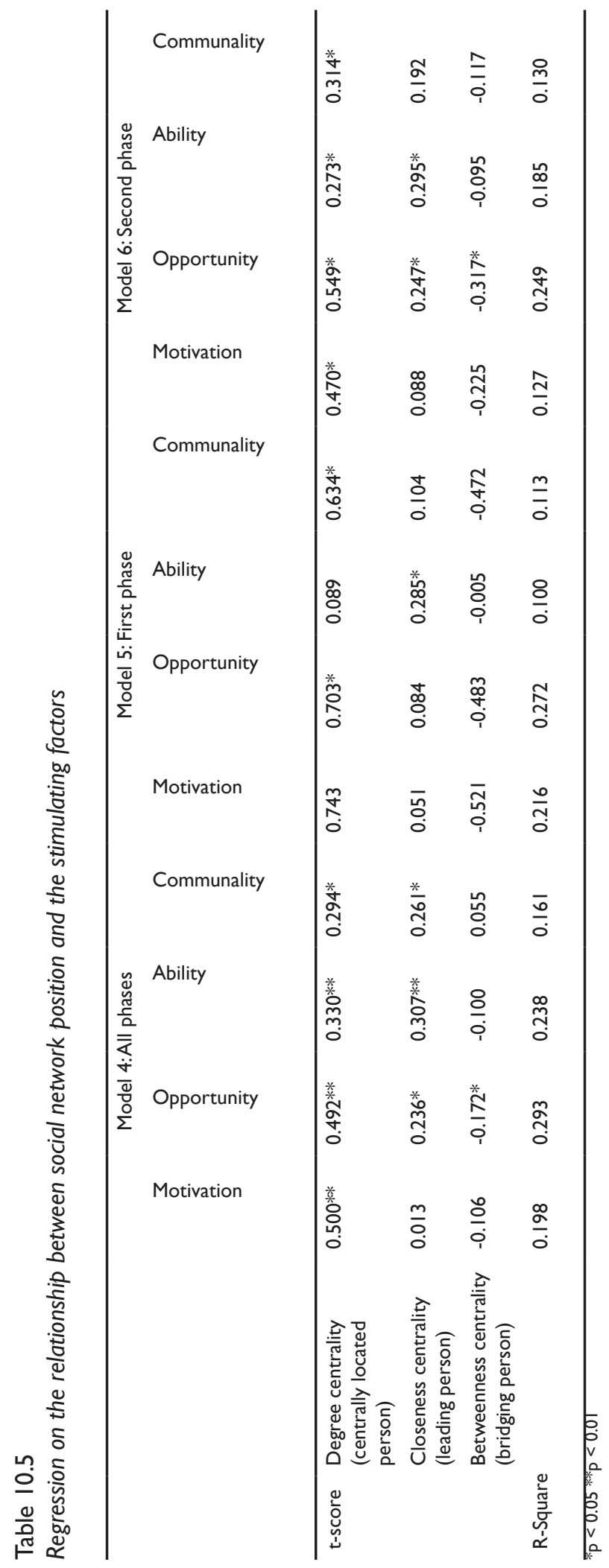


has been found between the betweenness centrality and one's opportunities $(B=-0.317, p<$ 0.05).

\subsection{Conclusion \& Discussion}

This research revolves around answering the following sub-question: What is the relationship between the formation of the social network positions and the motivation, opportunity, ability and communality? This study focuses on two components related to the central position: first, determining the social network position of an individual in a CoP and second, studying the relationship of the constructs in the MOAC model, motivation, opportunity, ability, and communality, with these social network positions. These two components were examined from two perspectives: first, the effect of the MOAC factors on the social network positions and second, the effect of social network positions on MOAC factors. These examinations were done by exploring the contribution of a social network position to the individual's motivation, reported opportunities, perceived ability and perceived communality. In addition to that, these two perspectives were analysed by using the complete dataset of four CoPs, as well as two subsets of these datasets: the first half of the CoP's duration and the second half of the CoP's duration.

The results in the first model show a positive significant relationship between motivation $(\mathrm{HIa})$ and opportunity $(\mathrm{H} 2 \mathrm{a})$ and the social network position of 'centrally located person', between motivation and the 'leading person' ( $\mathrm{HI}$ b) and between ability $(\mathrm{H} 3 \mathrm{c})$ and communality $(\mathrm{H} 4 \mathrm{c})$ and the 'bridging person'. Thus, the results show that degree centrality (centrally located person) is affected by the intensity of motivation. The higher the scores on the different types of motivation to participate, the higher the degree centrality. A plausible explanation for this is the fact that the more active a person is, the more convinced that person is of the added value of the CoP.The higher motivated people thus have more connections with people than do people who are less motivated. Another factor that positively relates to degree centrality is an individual's opportunity. Community members who perceive more opportunities to actively participate in a CoP, are more willing to interact with others.

The leading position of a person measured by his or her closeness centrality is largely influenced by their motivation. Community members in this network position in general have a higher motivation to exhibit the behaviour that leads to this position. The bridging person - who has an important position for transferring knowledge and information to others - are largely affected by their ability to share knowledge and information and the sense of communality that they perceive within the CoP.An individual's ability is largely influenced by his or her self-confidence and wide-ranging knowledge in a certain domain (Baran \& Cagiltay, 2010). These people are convinced of their own ability to support the development of the community and therefore act in this bridging manner between other community members. A plausible explanation for the relationship between the high ability of an individual and the high betweenness centrality of that individual lies in the fact that people who have a bonding nature know how to connect with others. These individuals can express themselves in a meaningful manner, know how to contact others and have the digital skills to act in a valuable way in online environments. There is also a positive significant relationship between the communality and the bridging position of an individual in a CoP.The fact that people with a high betweenness centrality score form the critical step between two others provide them with access to a variety of sub-communities. The development of a sense of community may therefore arise earlier. Bridging persons are important persons for distributing (new) knowledge to and through the network: Granovetter 
(1973) describes them as weak ties in a network. However, the explained variance in the last situation $(R 2=.072)$ is lower than in the other two situations $(R 2=.302$ and .212$)$.

In the first part of the discussion, we described the results of the first model representing the full network. In the continuation of the discussion we focus on the second model for the first phase and the third model for the second phase. In addition to that, the relationship between the network positions and the MOAC-variables will also be discussed in the models 4 to 6 . Due to the exploratory nature of the second part of this research resulting in the Models 2 and 3 and 5 and 6, no hypotheses have been formulated for these models.

In model 4, we found significant evidence for hypothesis $\mathrm{HId}$, which implies that people who are more centrally located are more motivated to act in a CoP. There is also evidence for the hypotheses $\mathrm{H} 2 \mathrm{~d}, \mathrm{H} 2 \mathrm{e}$ and $\mathrm{H} 2 \mathrm{f}$, these hypotheses are all connected to the individual's opportunities. The more central the position of a person is in a CoP, the higher his or her reported opportunities. The perception of an individual's ability is higher for the centrally located people ( $\mathrm{H} 3 \mathrm{~d})$ and for the leaders $(\mathrm{H} 3 \mathrm{e})$. Finally, the centrally located people and the leading persons have a higher sense of communality formalized as communality. These results reflect those of Dawson (2008) who also found that the degree centrality (centrally-located person) and closeness centrality (leading person) affect an individual's sense of communality. People with a position that is more leading or more centrally located position experience the community as a more communal environment. The striking results in this model are the relationships between the social network positions and the individual's opportunity and ability. People who have a more central position in a CoP are more positive about the opportunities they receive from, for example, their organisation. The people with more significant positions in the CoP also feels that they are quite able to contribute to the CoP in a meaningful manner. These perceptions of individuals regarding the factors in the MOAC-framework and their actual network position show a strongly polarizing development. A development that increasingly characterizes online communities (Bell, 2007).

Focusing specifically on the two phases in the CoP is focusing on the models 2, 3, 5, and 6. These models open the opportunity to obtain a clearer view of the role of the community process in the development and relationships in the CoPs. Models 2 and 3 show that an individual's motivation, the opportunities, and the perception of communality positively affect an individual's central location in the network of the CoP.The path coefficients and explained variance are also increasing from the first phase to the second phase. The increase in the coefficients explain how strong the effect is of one variable on another variable. The weight of the coefficients gives us an indication of the importance and thus enables us to rank the statistical value (Kwong-Kay Wong, 20I3). The ability is related to the leading position of a community member. This implies that the higher the ability, the higher their leading role in the CoP.The motivation of individuals has a rather strong relationship on the community members with a more bridging position in the CoP, this only applies to the second phase of the CoP. This could imply that community members who participate in the second part of the community process are more motivated to play an important role within the CoP.The relationships in the remainder of the two models are comparable.

Models 5 and 6 show more substantial differences between the relationships. In the first phase of the CoPs, significant positive relationships exist between the centrally located persons and their reported opportunities and communalities between the community members. The leading person positively relates to the individual's ability, so the shorter the paths from an actor to all 
the other actors, the higher their self-efficacy. In the second phase, more significant relations are found. The more centrally a person is located in the CoP, the higher their motivation, reported opportunities, perception of their ability and sense of community. Further on, the more leading a person is in the network, the higher their reported opportunity and their ability. An increase in the bridging position result in a decrease in their opportunities. So, people who take a more bridging role in the CoP, are more negative about their opportunities. A possible explanation for this might be that the community members with a high betweenness centrality score are not fully embedded in a community. They connect different communities, but probably do not feel a member of any of them, they also do not feel supported by the organisation to eventually feel this way.

The link between actual behaviour (the web-based social interaction data) and the explanatory variables (the survey) from the MOAC model provides support in explaining an individual's network position in a CoP. Following the community analytics in the CMEM-depicted in Chapter 5-this approach contributes to the construction of descriptive and diagnostic community analytics. This study forms a first step towards merging objective data shown as an individual's actual behaviour, with subjective data, tested via their motivation, opportunities, abilities and communality. The results in this study yields new insights into the role of network positions and de development of these positions. However, due to exploratory nature it is highly recommended to use this study and its approach as a starting point for improving the methods to evaluate social network positions and explaining them by using additional resources. By dividing the research data into multiple models, we have made a first step towards investigating network positions over a certain period. Especially when we are able to collect and store sequential data (e.g. comment on a comment) in a adequate way, we will be able to determine even more precise and accurate the individual network positions. However, generally speaking the ever-increasing number and size of datasets and the possibilities to visualize these developments create promising possibilities for real-time monitoring and network positions. 



\section{1}

\section{Social Interaction Patterns in Communities of Practice}

\section{II.I. Introduction}

This chapter focuses on identifying and evaluating social interaction patterns in the CoPs by extracting the patterns in the network structures of these CoPs. In this study, the same webbased social interaction data has been used and processed for analysis as in the preceding chapters. However, in this case the focus is on the generation of bipartite networks of community members and the types of social interactions they engage in. These data offer unique opportunities to understand the social processes in CoPs. In the previous chapter, the combination of survey data with web-based social interaction data has been used to examine network positions in CoPs. In this chapter, another perspective has been chosen to investigate the development of CoPs. By merging the web-based social interaction data with survey data, we can explore the structural mechanisms that shape how community members engage in CoPs. In this study, the first steps are to seize and capitalize on the value of these datasets and to study how these communities have developed based on these datasets. By drawing on the two-mode networks of 125 community members and 5 types of social interactions generated from four CoPs in as many organisations, we examine structural logistics and individual stimulating factors that stimulate the creation of different types of social interaction. All of this is done with the following sub-question in mind:What structural processes and individual-level factors shape the network structures in communities of practice?

\section{II.2. Theory}

Ideally, CoPs are formed independently and organically without the involvement of, for example, organisations. However, in practice, it is not that simple. CoPs are frequently sponsored as subcommunities within larger organisations as a means of promoting knowledge exchange and collaboration among professionals who have a similar profession or practice. In many CoPs, specific persons are assigned to take certain roles in the community-for example, community managers and ambassadors. The more successful and interactive CoPs evolve more organically than the less interactive groups; these must be stimulated by community managers and other peers (Jiménez-Zarco et al., 2014). In general, the CoPs help practitioners to make sense of concrete information (e.g., practice guidelines, trends and developments) in the context where it is used. A good understanding of the developments in these CoPs supports, for example, community managers, information managers and communication experts in ensuring the management of a continuous and up-to-date monitoring process. In this theory section, the structural effects and individual attribute effects relevant for analyzing the behavior in CoPs are described in more detail.

\section{II.2.I. Structural effects}

CoPs are initiated as an online meeting point for professionals in a variety of organisations to collaboratively improve and innovate their practices. The social technologies developed to 
support this objective often offer a variety of functionalities to stimulate social interaction between community members. In this chapter, we approach social interactions from a sociological perspective and therefore introduce some relevant social network concepts.

A social network is "a social structure consisting of a set of actors (such as individuals or organisations) and a set of dyadic ties between these actors" (Wasserman \& Faust, 1994). These dyadic ties occur in different capacities ranging from knowledge exchange to appreciation to social relations. In large populations such as networks or communities, socially connected individuals often have correlated behavioural patterns, often seen from the perspective of network dependency (Lane, Pengyu, Zhou, \& Zhao, 20l4). Numerous social processes affect these network dependencies, including social influence (Robins, Pattison, \& Elliott, 200I) and social selection (Robins, Elliott \& Pattison, 200I). However, social processes affect network dependencies, and specific individual attributes affect these dependencies (e.g. age, gender, political preference).

\section{Power law}

The power law (Hindman, 2009; Pareto, 1896) or Matthew effect (Merton, 1968) is a term used for the same phenomenon, that describes and validates, in more common language, how "the rich get richer and the poor get poorer". Merton (1968) has identified the Matthew effect in the sociology of science as the fact that renowned academics often receive more credit for their work than a researcher from a lesser-known institution does, even if both academics' work is completely comparable. This implies that in general, credit will usually be given to those researchers who already have a central position in their domain. By comparing this phenomenon with crowdsourcing behaviour, it becomes clear that only a few people create the majority of ideas (Stephens, Chen, \& Butler, 2016). Network centrality measurement is a measure for identifying and determining how unevenly centrality is distributed in a network. The less active members of a community are mainly situated in the peripheral areas of a network, while the active members are located in the centre of a network (Lundkvist, 2004). It is assumed that the composition of the underlying networks in CoPs is increasingly based on power law distributions and less on normal distributions.

HI: A few community members start many interactions while many community members start a few interactions

\section{Shared affiliation}

Focusing on the social structures in a CoP results in attention for the shared affiliation effect or alternating-two-paths (Wang et al., 20I3). Zappa \& Lomi (20I6, p.345) explain that the shared affiliation phenomenon originates from "affiliation-based closure arc tests". This implies that community members are more likely to interact with other community members who have a commonality. In the study of Wang et al (20I3), this was shown by the tendency of youngsters who co-attend one event to co-attend multiple events. This results in a set of young people who co-attend a series of events, and another group who also co-attend another series of events. It is assumed that a positive tendency will be found for community members who co-initiate one type of social interaction to co-initiate more types of social interactions. This results in the following hypothesis:

H2: Community members who both initiate one type of social interaction are more likely to start more types of social interaction 


\section{I.2.2. Individual Attribute Effects}

The individual attribute effects in this study are used to analyse the possible relationship between the individual attributes and the presence of social ties. This fits seamlessly with the exponential random graph modelling technique in which actor attributes are treated as exogenous or explanatory variables that affect the presence of social ties.

\section{An individual's motivation}

Professionals who are highly committed to the purposes of an organisation are more willing to share their knowledge (B. van den Hooff \& de Leeuw van Weenen, 2004). This fact is also likely to apply to the commitment of individuals to CoPs in organisations and their active behaviour in these communities. Individual commitment stems from their motivation to perform in a certain manner, the more diverse and intense their types of motivation, the more positive their perception regarding the CoP (Chen et al., 20I3). A considerable number of motivations lie at the basis of the decision to participate in a CoP. Particularly in the context of group behaviour, some motivations are primarily important because they are related to knowledge exchange, knowledge acquisition and social interaction: being informed (Hara \& Hew, 2007; Lee \& Ma, 20I2), improving work performance (Bock, Orces, Kim, \& Lee, 2005; Han et al., 2007; Kulkarni et al., 2007), improving social reputation (Bettiga et al., 20I8; Kulkarni et al., 2007) and subjective norms operationalized as social pressure (Lewis, Agarwal, \& Sambamurthy, 2003; Scarbrough, 2003). Together, these different reasons form individual motivation which leads to the following hypothesis:

H3: Community members with a high level of motivation to participate in a CoP are more likely to reciprocate their type of social interaction with other community members.

\section{An individual's opportunities}

Individuals' motivations to act are often strongly interrelated with the opportunities they receive from organisational management, their colleagues, or themselves. One of the first steps when designing a CoP is convincing organisational management of the added value of a CoP for their organisation, the CoP is strongly dependent on the financial support of the organisation with regard to the continuity of the CoP (Verburg \& Andriessen, 2006). Opportunity-a component in the motivation, opportunity and ability model (Macinnis et al., 1991) — represents the extent to which the situation is conductive to accomplishing a particular action, and it includes the contextual factors that facilitate or hamper certain behaviour. Fadel \& Durcikova (20I4, p. 3606) describe opportunity as the "presence of enabling environmental mechanisms that facilitate task performance". Factors such as organisational norms, intellectual demands of the task, facilitating conditions or other climate factors can be viewed as enabling environmental mechanisms to support knowledge exchange in CoPs and are therefore categorized as opportunity variables (Fadel \& Durcikova, 20I4). If a community member wants to learn, it is crucial that he or she has the opportunity to do so. This means that an organisation should facilitate and support its colleagues in participating in an active matter. Three of the factors which are important when speaking about the opportunity to stimulate group behaviour in CoPs are: time (Orlikowski, 1993), work autonomy (Cardona, Lawrence \& Bentler, 2003; Salanova, Peiró, \& Schaufeli, 2002) and facilitating conditions (Bhattacherjee, 200I;Venkatesh, Morris, Davis \& Davis, 2003). To help individuals gain their optimal strength, it is crucial to offer them the opportunities they need. 
This results in the following hypothesis:

H4: The more opportunities a community member has, the higher the level of reciprocity in his or her types of social interaction.

An individual's ability

Communication media, which are fundamental for online-based CoPs, have enhanced individuals' capacity to find information that is pertinent to the performance of their daily tasks (Malone, 2002). Ability can be seen as the extent to which an individual possesses the necessary skills and abilities required to commit an action (Macinnis et al., 199I). Wasko \& Faraj (2005) have found evidence that individuals with higher levels of expertise are more likely to provide useful advice and share relevant knowledge than people who feel that their expertise is inadequate. Therefore, an individual's perception that he or she can contribute knowledge, expertise and skills to the community is an indicator of the actual intensity of that person's social behaviour.

To behave in a meaningful matter, it is crucial to have the skills and confidence to do so. Siemsen et al., (2008) and Turner \& Pennington (20I5) recognize an individual's ability to express him or herself in a meaningful manner as one of the key components in the exchange of knowledge. Siemsen et al. (2008) define this as the extent to which skills and proficiencies are required to share knowledge with co-workers. Group behaviour is also a matter of interaction and therefore of contacting others; Cadiz et al. (2009) define such behaviour as social networking skills, while Venkatesh et al. (2003) and Tseng \& Kuo, (20l4) mention digital skills as crucial skills for meaningful social behaviour in CoPs. Thus, the ability-which is often operationalized as selfefficacy — of an individual to act in a certain manner is a strong indicator for that individual's type of social interaction and the intensity of interactions, which results in the following hypothesis.

H5: Community members with a high ability to participate in a CoP are more likely to reciprocate the types of social interaction.

\section{An individual's perception of community}

The sense of community in a CoP is seen as an important indicator for a healthy community (Talò, Mannarini, \& Rochira, 20I4). Nistor, Daxecker, Stanciu, \& Diekamp (20I5) define sense of community as "the socio-emotional quality of communities". It is crucial for community members to be committed to the norms and values of the community. Indicators for a sense of community are the presence of a shared language (Chang \& Chuang, 20I I), shared leadership (Johnson et al., 20I5) and shared identity (Kimmerle et al., 20I3). Increasing the emotional value of these indicators for individuals helps to stimulate the sense of belonging to the community (or sense of communality). It is therefore very likely that community members with a higher sense of community will be more active in the community than community members with a lower sense of community. This statement is also confirmed by Talò, Mannarini, \& Rochira (20l4), who studied the relationship between sense of community and social behaviour in communities. They found a significant, positive and moderately strong relationship between different types of social behaviour in the adult population and specific cultural contexts. This brings us to the following hypothesis.

H6: Community members with a high sense of communality are more likely to reciprocate the types of social interaction. 


\section{I.3. Data and Methods}

The methods and techniques that will be used in this study to provide a reliable picture of the social interaction patterns within a CoP form an important part of the community monitoring and evaluation methodology (CMEM), since the CMEM provides insight into descriptive, diagnostic, and prescriptive community analytics, which are vital analytics for providing a good picture of developments within CoPs and the underlying stimuli.

The first step in this study entails the survey with community members to identify factors that are expected to affect their social behaviour. The survey used for this purpose was introduced and tested in Chapters 6 through 8 . In this chapter, the same connection has been made as in the previous chapter between the survey data and the web-based social interaction data gathered from the online platform. The respondents were asked to give their permission for connecting their answers to a web-based social interaction dataset before we connected the data. Only those respondents who gave approval for connecting their survey data with their online behaviour in the CoP were incorporated in this research. By giving their full name and email address, respondents enabled us to merge the community data with the survey data. After merging the datasets, all traceable data were deleted to ensure anonymity and guarantee that ethical standards were respected. The final document has only unique identifiers without further background information for all participating community members.

The online survey was designed to gather the perceptions of community members regarding their social behaviour in CoPs. In this study_conducted from December 2015 to April 2016community members were asked for their individual motivations for social behaviour, the reported opportunities offered to them by their organisation, their perceived ability to perform and their perception of communality. In addition, the respondents gave their demographic data such as age, gender, job function and experience.

The web-based social interaction dataset consists of data that represent the relation of an individual community member with a certain type of social interaction (e.g., creating a post or commenting on a post). Since this research has an explorative character, we decided to explore the developments in CoPs from a network perspective. Bipartite graphs and attributes from the survey were used to study the relationship between the social structures in the CoPs and the factors that affect these structures. Every time a community member interacts in an online CoP-be that by writing a post, commenting on a post or even logging into the virtual space-the member creates a digital footprint. Community analytics is the process of using the data from these footprints to improve the group behaviour potential. The webbased social interaction data consisted of numerous types of social interaction. In total, the I 44 members created 5.437 posts and 7.739 comments, sent 3.393 tips and 5.708 likes and started 1.800 chats. All interactions of an individual community member were registered but unstructured. Following the data-driven process, additional data cleaning and conversion were required and conducted to create individual profiles. These individual profiles can be connected to the responses these users gave to a survey. This survey aimed to identify those factors that explain the online behaviour of individuals in CoPs. The motivations, reported opportunities, perceived abilities and perceived communalities associated with these CoPs form the basis for this formative evaluation.

This combination of survey results with web-based social interaction data provides a unique opportunity to analyse the social network by using the survey results as attributes for the 
individual actors in the network, a new approach to reducing the distance between objectivity and subjectivity. In this case, the attributes form an indicator or exogenous variable that might be an indicator for the structural development (endogenous variable) of the bipartite network graphs.

\section{II.3.I. Case description}

Four organisations approved the use of web-based social interaction data from their online community software for research purposes. We analysed data from a time period ranging from 17 to 33 months from four CoPs. All members of these CoPs were invited to participate in a survey studying the factors that affect their social behaviour in the CoPs. As already indicated, a total of 144 community members agreed for their survey response to be used in combination with their behaviour in the CoP. Due to missing values, a total of 125 participants were ultimately incorporated in this study. This offers several opportunities to examine the developments within CoPs. These four CoPs - as described in detail in the chapters 9 and $10-$ make use of these same online community technology, which ensures that the cases - despite the domain-specific differences - are comparable in the types of behaviour. Each member in the CoP voluntarily created a personal profile with his or her real name.

\section{II.3.2. Endogenous variables}

The endogenous variables were estimated from network statistics computed by counting the number of specific local network configurations in the observed network (Wang, Robins, 2009). A proven statistical method to analyse these local network configurations is introduced by Wasserman \& Pattison (1996); these authors allow global network structure to be understood by considering and focusing on local processes.

\section{Statistical analysis bipartite network graphs}

Social systems are complex interaction networks where nodes represent the entities (e.g. individuals, organisations, groups) and edges characterize the relations or interactions among them. These social networks arise from a wide variety of scientific domains such as sociology, communication and management. Online social platforms such as Facebook, Linkedln and Instagram are examples of interesting (semi-)public interaction networks, while organisations increasingly start their own internal social knowledge networks to stimulate the interactions between colleagues. The study of the underlying complex networks can provide insight into their structure, properties and behaviour.

In this study, a comparable perspective is chosen, as introduced in the work of Stephens, Chen, \& Butler (2016). These authors have studied the development of social interactions in organisations by framing it as inter-organisational crowdsourcing and used the perspective of bipartite network analysis. The main purpose of this approach is to obtain a better understanding of the structural processes driving idea challenges that stimulate the generation and selection of ideas. More specifically, an exponential random graph model (ERGM) has been created to handle bipartite network data structures. The purpose of an ERGM is to understand what type of tendencies or forces might affect the structure of an observed network (Pilny \& Proulx, 2017). ERGMs represent statistical models specifically designed to analyse data about all kinds of networks, such as biological, computational and social networks.

Usually, ERGMs are focused on one-mode networks in order to examine local processes 
such as reciprocity, transitivity and homophily (Lusher, Koskinen \& Robins, 2013). However, methodological advances have resulted in an extension of the perspectives and possibilities of (social) network analysis. Network researchers such as Agneessens \& Roose (20l7) and Wang, Pattison, \& Robins, (2013) have extended the ERGMs by adding possibilities to analyse clusters, node attributes, and bipartite networks. Wang, Pattison, \& Robins (20I3) proposed a set of new local configurations for ERGM specifications that are relevant for bipartite networks, enabling scholars to study new logics that create the overall bipartite network structure.

We aim to understand which attributes explain the structure of a given network. An exponential random graph modelling approach is used to study this phenomenon. Standard regression modelling techniques are focused on the independence of variables, while it is the lack of independence that makes a network. As discussed by Asur, Parthasarathy, \& Ucar (20I5), interaction networks have often a modular setting. By identifying the modularity represented in common patterns in the interactions between nodes, certain clusters or communities can be defined. These clusters or communities represent a group of people with similar interests, contacts or professions.

For the purpose of this study, we have looked at networks from two perspectives: social structure effects and attribute effects. The social structures in the network were analysed to determine whether there is a social affiliation effect and a spread of activity. These two forms were related to the first and second hypotheses. To determine whether two persons with the same attribute start comparable types of social interactions, we merged the survey data containing the motivational factors with the social network data. These analyses were conducted to provide answers to hypotheses 3 to 6 .

The bipartite network has two distinct sets of nodes, and ties are defined between only the nodes from these different sets (Wang et al., 20l3). The focal points of the analysis were four matrices: the first matrix shows the whole network of social interactions; in the second matrix, the focus is on the most active posters; the third matrix shows a moderate group of posters; and the fourth matrix gives insight into the least active posters in a community. The composition of these groups is based on the weighted scores that they have in relation to each other. This decision was made to prevent the more active organisations from having a greater influence on the results. Each matrix has the same structure, with $x=[x i j]$ and with a model-dependent number of rows (professionals) and $\mathrm{m}=5$ columns (type of social interaction). If the ith community member started an interaction with the jth type of social interaction, then the cell $\mathrm{xij}=$ $\mathrm{I}$; otherwise, $\mathrm{xij}=0$. If the ith professional started a certain type of social interaction more often, the value in the cell increases. We understood the observed network $x$ as one possible realization of set $X$ of random tie variables $\mathrm{Xij}$, thus connecting the data structure to the bipartite ERGM (Wang et al., 20I3). "The purpose of ERGM is to figure out what type of tendencies or forces might

Table II.I

Composition of the survey

\begin{tabular}{ll} 
Construct & Number of items \\
\hline Motivation & I5 items (ordinal) \\
Opportunity & 5 items (ordinal) \\
Ability & 9 items (ordinal) \\
Communality & 7 items (ordinal) \\
Age & I item (nominal) \\
Organisation & I item (nominal) \\
\hline
\end{tabular}


be responsible an observed network looking the way it does" (Pilny \& Proulx, 2017, p.7). The ERGM offers insight into network properties through the simultaneous inclusion of structural parameters and attributes in testing observed data with simulated data while accounting for the interdependent nature of the data. The logic behind it is similar to the logistic regression approach (Harris, 20I8).

A model with variables such as age and gender are proposed to predict the presence or absence of a dependent variable. For the logistic regression, this implies that the model represents a curve, and in ERGM analysis, the model represents an actual network. The main difference is that since ERGM attempts to create a network, each specified effect is not independent from the others (i.e., a high value in one effect might inherently mean a low value for another), which is why ERGM uses such a complex combination of simulation and inferential statistics (Pilny \& Proulx, 2017). To check model adequacy, a goodness of fit (GOF) assessment will be conducted. GOF aims to answer the question whether the simulated networked created by the ERGM formula resembles to the observed network. The ERGM simulates thousands of networks and compares the average statistics of those networks with the observed version. This ultimately leads to an identification of the places where the scores look similar and places where they look different. The higher the similarity, the better the model fit.

The exogenous variables or attributes applied to the social network datasets are age, organisation, and scores on 'motivation', 'opportunity', 'ability' and 'communality'.

\section{I.3.3. Exogenous variables}

The exogenous variables-often called the attribution effects or exogenous actor covariate effects - indicate whether a network develops in a certain direction. In this study, the network data from four CoPs were merged with the results of an extensive survey. The constructs used in this chapter are already examined in Chapter 10. This has led to the same variables used to measure the four constructs (Table II.I). The variables that we not included in the definition of scales for motivation, opportunity, ability and communality in Chapter 10 are also excluded in this chapter (see Appendix 10.1 and Appendix 10.2).

\section{II.3.4. Software}

The unstructured form of the data requires a multiplicity of software packages. The web-based social interaction data were collected in MySQL; cleaned, transformed and processed in Excel; and analysed with MPNET. The survey data were analysed by using SPSS, SmartPLS, and the combination of both data sources with the ERGM software package MPNET-developed by Wang, Robins and Pattison (2009) — was used to accommodate bipartite network tie types, as is needed to examine the relationship between people and their type of social interaction in CoPs. MPNET is a proven, powerful tool for the statistical analysis of, among others, one-mode networks, multilevel networks and bipartite social networks.

\section{I.3.5. Research Reliability and Validity}

Scientific research ultimately always depends on valid and reliable research methods, and this combination guarantees both scientific value and meaning. In assuring the reliability and validity of this research, several measures have been applied. Reliability improvement has been realized by treating all CoPs equally. Since CoPs are complex, to guarantee the validity of the research, 
we adopted a multi-method approach (Mccully \& Lampe, 20II). In this technique, a certain situation will be observed from many complementary perspectives, and relevant observations and explanations will be collected. In this field of research, the study of community development is common. Community development is analysed by using survey data merged with web-based social interaction data to obtain a clear picture of how and why communities evolve in a certain manner.

\section{II.3.6. Analysis}

Network analysis is a systematic approach for capturing the relations among a given set of nodes. One of the more advanced statistical models used to analyse the evolution of networks is the exponential random graph model (ERGM), also called a $\mathrm{p}^{*}$ model (Lusher et al., 20I3). ERGMs provide a methodology for investigating network structures and processes empirically. Standard statistical methods such as t-tests, analysis of variance (ANOVAs), and regressions assume independence between variables, whereas ERGM focuses on (social) networks, where variables often depend on each other. Because an ERGM can incorporate dependence, it is a more principled approach to understanding the patterns of network ties. The attributes related to individual nodes determine the interrelationship between the nodes.

\section{I.4. Results}

\section{II.4.I. Bipartite networks of group behaviour in CoPs}

Visualizing bipartite networks offers many opportunities. It provides insight into the diverse member types, key players and the most popular types of social interaction. Figure II.I shows the bipartite network for the four communities, only those community members who gave permission to merge their survey responses with the web-based social interaction data are shown. The figure shows that a community member (circle) creates a certain type of social interaction (square). The size of the circles describes the number of social interactions that the person started, the size of the relationships describes the number of times an individual creates a specific type of social interaction.

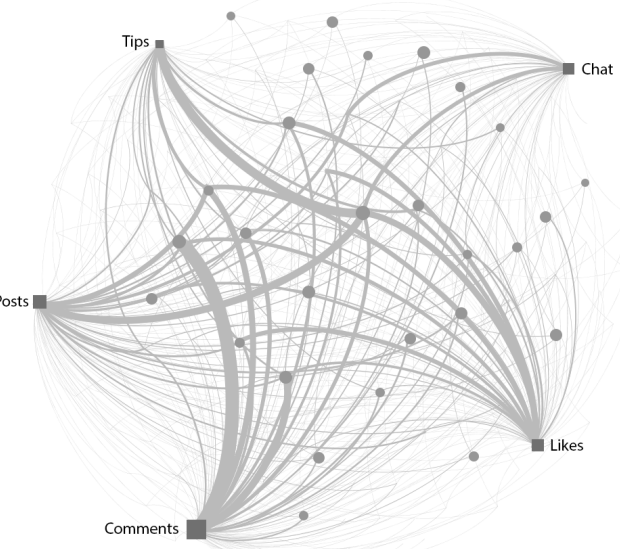

MPNET is used to define and analyse the Figure II.I. Bipartite network community network structures in the CoPs. Originally, behaviour

the data represented relationships between

an individual community member and his or her unique action within the CoP; actions are embodied by, for example, creating a comment on post $X$. Since not all community members gave permission to merge their survey responses with the web-based social interaction, analysing networks with these members would result in many isolates. Therefore, we have decided to summarize the unique social interactions as just the type of social interaction. This means that instead of person $A$ creating a comment on post $X$, the relationship is that person $A$ creates a comment. In this study, we used an approach with one type of attribute. The nodal attributes, 


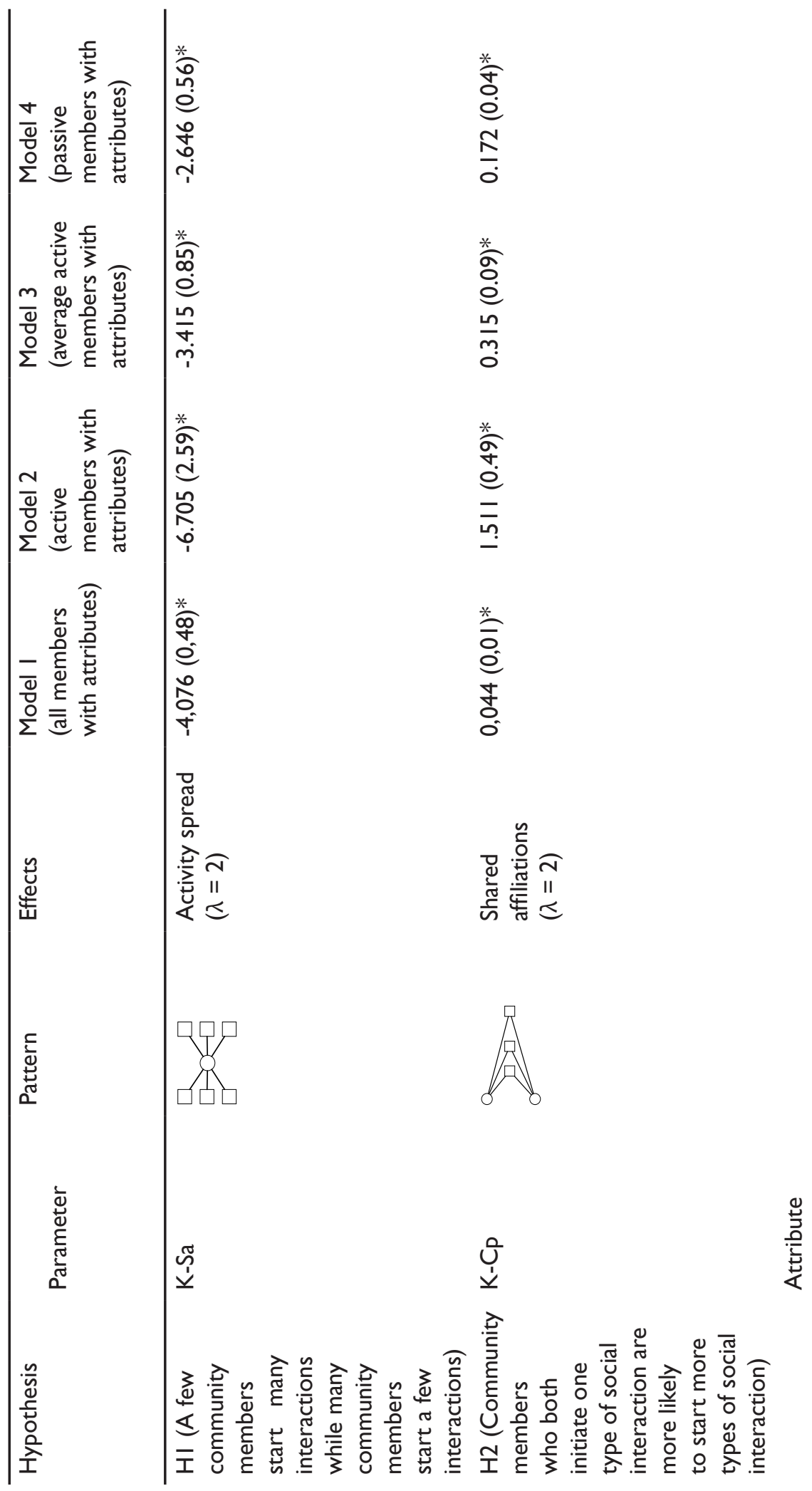




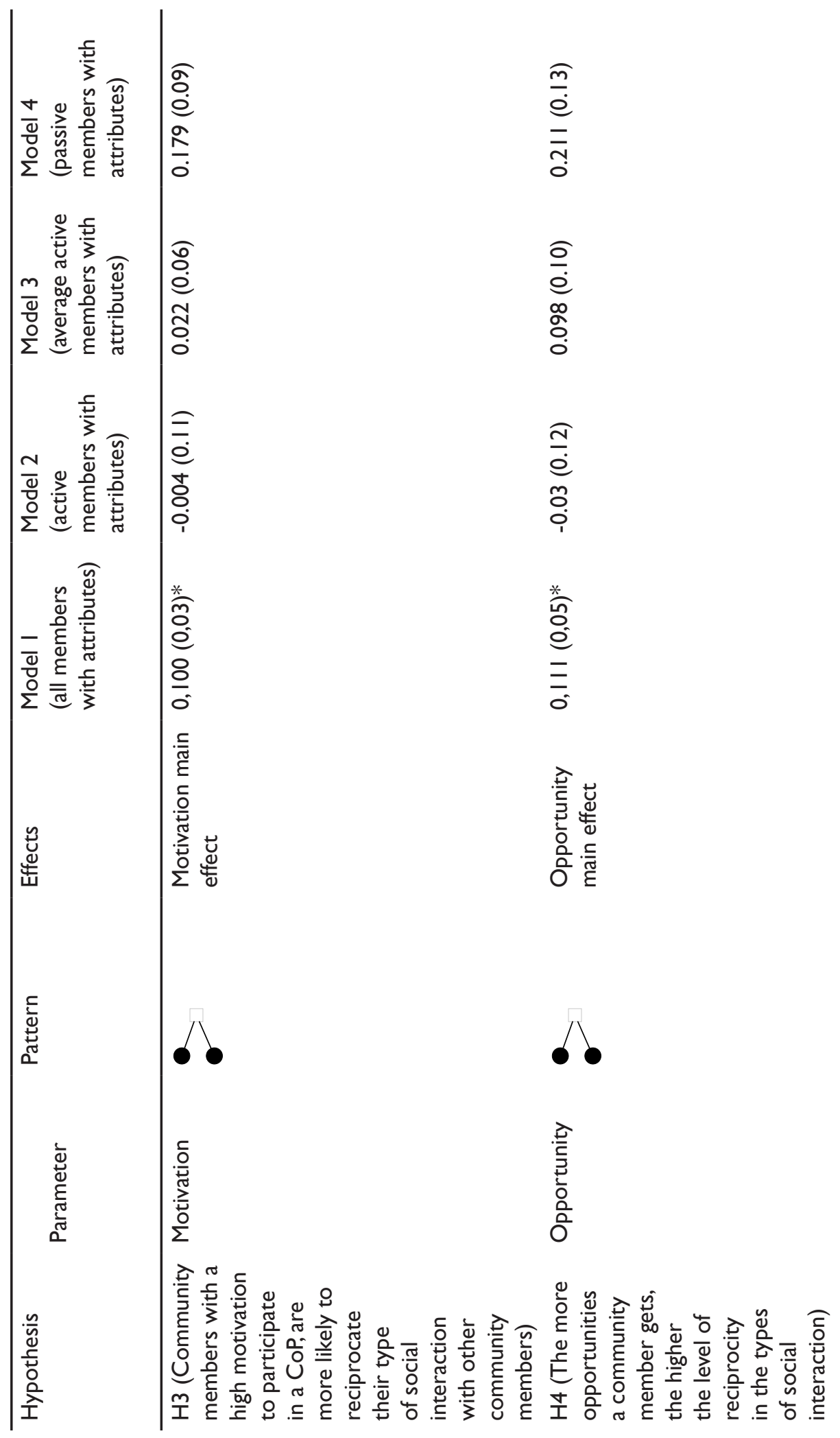




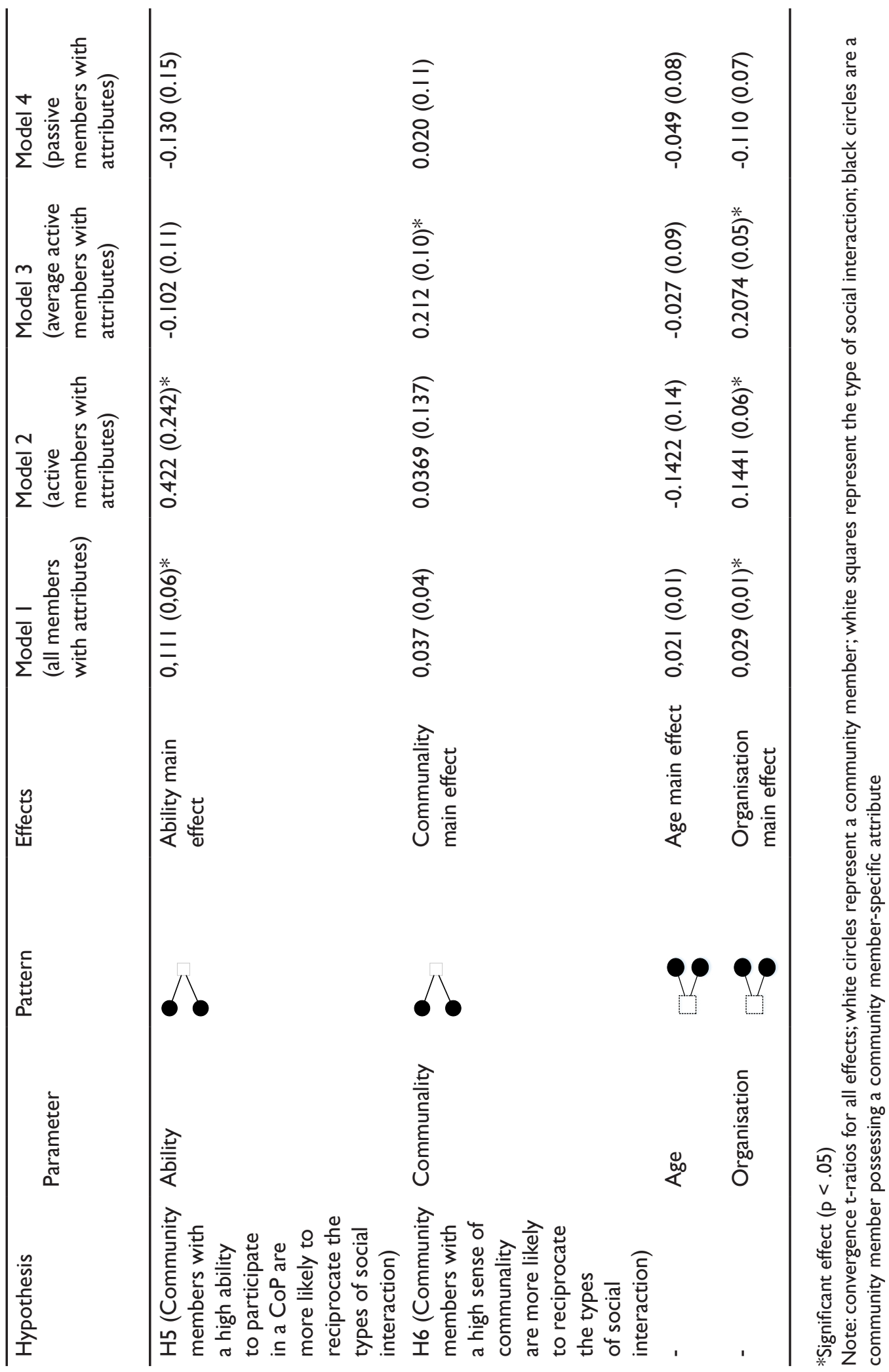


which are formed by the exogenous variables from a survey. These exogenous variables are the motivation, opportunity and ability to participate and the communality the community members perceive regarding the community to which they belong. The web-based social interaction data and the survey data provided insight into various characteristics of the community members that might affect the type of social interaction. This insight offers possibilities for predictions and interventions of social behaviour. When certain characteristics of community members are known, we will be able to predict what they might do and how we can intervene to steer this behaviour in a certain direction.

\section{I.4.2. Structural effects}

In Table I I.2, we reported estimates from models I, 2, 3 and 4. Since this study is exploratory in nature, the large differences in the intensity of social behaviour led us to develop models for the different levels of activity (active, moderately active and passive social behaviour) in addition to an overarching model. This will enable us to provide a better picture of behaviour by the level of intensity. These models were specified with two structural configurations and have a good overall heuristic measure of model goodness-of-fit (GOF). We found a significant effect for hypothesis I in all models, demonstrated with negative, significant estimates for a community activity spread effect [K-Sa] $(-4.08,-6.7 \mathrm{I},-3.42,-2.65, \mathrm{p}<.05)$, indicating low variations in the degree distributions of the community members regarding starting different types of social interactions. This implies that there are several active community members and just a small number of community members who participate rather passively. Therefore, the vast majority of the participants in a CoP have entered into some type of social interaction at least once. There is also support for hypothesis 2 which indicates that community members who start one type of social interaction are more likely to start more types of social interaction [K-Cp] (0.04, I,5 I, 032, 0, I7, $\mathrm{p}<.05)$.

\section{I.4.3. Attribute effects}

Focusing on the attribute effects, we tested the hypotheses for all four models. The result of this is that the chance that each hypothesis fits with the models is rather small. However, for the full model (model I) we found evidence for hypotheses 3,4 and 5. In the case of hypothesis 3 , this implies that considering the complete sample of community members, there is a small but positive association $(0,100, p<.05)$ between the level of motivation and the amount of different types of social interaction that are entered into. For hypothesis 4 , a positive linkage $(0, \mathrm{II} I, \mathrm{p}<.05)$ is identified between the opportunities one gets and the amount of different social interaction types a community member initiates. For hypothesis 5 , an individual's ability has a positive relation $(0,1 \mathrm{II}, \mathrm{p}<.05)$ with the variety of social interaction types that the community member initiates. However, hypotheses 3 and 4 should be rejected in models 2,3 and 4. Hypothesis 5 is accepted for model $2(0.422, p<.05)$ and hypothesis $6(0.212, p<.05)$ is accepted for model 3. In all other cases, the hypotheses are rejected for the corresponding models. Since the dataset consisted of four organisations, we also decided to keep it as an attribute in the analysis. In models I, 2 and 3 this resulted in a matching organisation homophily (2-path connection; $0.028,0.151 \& 0.205$ ). The principle of homophily holds that people associate with other groups of people who are most like themselves. In an organisational sense, this implies that the organisations with the more active communities have community members who initiate similar types of social interaction. 


\section{I.4.4. Goodness of fit}

A goodness-of-fit (GOF) assessment is conducted to test whether the simulated networks are close to our observed networks. To check for this model adequacy, goodness of fit (GOF) tests were conducted which are based on a simulation approach. For the purpose of this study, a total of 10 million iterations are simulated, sampling every thousandth network and then counted the number of predefined structural configurations for the sample graphs in order to create distributions of graph statistics. T-statistics were calculated for each relevant observed graph statistic based on the mean and standard deviation from the simulated distribution. The GOF test takes a closer look at the similarities and differences between the two types of models. The more similar the scores in both networks look, the better the model fit. All four models in this research show a fairly good fit since the t-scores are all below 2 .

\section{I.5. Conclusions \& Discussion}

This study analyses structural processes and individual-level motivational factors that shape the network structure of group behaviour in CoPs. Coming back to our research question formulated in the beginning of this chapter-What structural processes and individual-level factors shape the network structures in communities of practice? - a number of steps were set to answer this question. First, just like in Chapter 10, the results from the survey have been merged with the web-based social interaction data that represents the actual behaviour of the respondents in the community. This offered us a unique opportunity to examine the social structures in CoPs from another perspective than purely focusing on one type of dataset. Bipartite ERGMs were drawn to explain the observed patterns of dual associations between community members and types of social interactions through local network processes. In addition to that the motivational factors were used to analyse the role of these factors in shaping the network structure for social interaction types. This analysis with ERGM-application in combination with motivational factors is a unique first attempt to link different research methods together to increase the triangulation and better explain the network patterns. The community platforms capture real-time digital trace data from all community members' social behaviour, which enables us to analyse the bipartite relations for the entire duration of each CoP. This work thus enriches the handful of existing studies of bipartite ERGMs. Both perspectives result in some interesting findings.

The results show that in all communities a substantial group of people engage in many types of social interactions and that in the communities the number of activities is negatively spread through the network. This indicates that the types of social interactions are less widespread across the CoP than we initially assumed. There is large group of community members - who are in the sample - that initiate a lot of different types of social interactions, while a small group is only entering a limited number of social interaction types. This implies that the community members know the technical possibilities of the online community software, but that - in the light of the activity levels shown in the previous chapters - these opportunities are not being fully exploited. In relation to the attributes connected to all individuals, we found significant relations for the full model (model I) between community member's level of motivation, level of opportunities and ability to perform and the number of social activity types they are involved in. In the case of predominantly active community members (model 2), there is also a significant association between the ability to perform and the number of different types of social interactions one starts. When an individual has a higher self-efficacy (ability), he or she is more likely to start in different types of social interaction. When an individual's self-efficacy 
is lower, he or she is also involved in fewer types of social interaction. It is remarkable that in model 3 - with the group of moderately active people - a significant positive relationship has been found between the sense of community (communality) and the initiative-taking behaviour regarding the different types of social interaction. This was especially expected in model 2 with the predominantly active community members.A possible explanation for this might be that the active group of participants in this case approach the CoP primarily as a means for knowledge management and less to jointly improve / develop a practice. The smaller active group may focus more on the development of high-quality discussions

This research demonstrates how social structures underlying group behaviour in CoPs help to identify significant social interaction patterns in CoPs. From a practical perspective, this implies that individuals with a higher level of motivation, who experience more opportunities and can act in a meaningful manner, are more inclined to engage in different types of social interaction. The hypothesis that individuals with a greater sense of communality are more inclined to fully embrace the possibilities of online community software is not significantly proven, but nevertheless there is a small positive relationship in its favour. In short, it is very important to increase the individual motivation in order to support the development of CoPs.An organisation can be supportive by providing opportunities (time, work autonomy or facilitating conditions) or by focusing on improving abilities (by means of training, coaching or (technical) support).

From an academic perspective, this research approach, whereby the results of a survey were directly connected to web-based social interaction data, is a unique approach to increase the overall reliability and validity of explanations of social behaviour. This work is one of the first attempts to merge two different disciplines of academic research: communication science and sociology. In communication science, the focus is predominantly on the form, reasoning and consequences of communication, and in sociology the focus is on the study of society, including patterns of social relationships and social interaction. In this study, the reasoning for social interactions was analysed by using the specific statistical inferential social network method ERGM.

Returning to the positioning of the methods and techniques used in this study within CMEM, we can conclude that these methods and techniques provide valuable insight into social interaction patterns. These patterns can form the basis for improved insight into predictive and prescriptive models, allowing for more targeted interventions. Recurring patterns provide a breeding ground for predictions and, ultimately, prescriptions. As in chapters 9 and 10, more data will lead to more valid insight into network configurations based on structural patterns.

The explorative character of this research also automatically leads to some limitations; therefore, it must be interpreted as an important first step in the design of a multi-method approach for the evaluation of social interaction patterns. As is normally with survey research, a limited number of respondents participated in the survey and an even smaller group gave approval for linking their answers to the web-based social interaction data. One consequence of this is that one-mode networks are difficult to develop, given that many attribute variables are missing. We had access to their network data, but we were not able to connect it directly to the motivating factors. Proceeding with a social network with only those community members who gave permission for merging their community behaviour with the survey data would have automatically resulted in many isolates. We therefore decided to analyse the data from a bipartite perspective by focusing on the social structures consisting of the community members on the one hand and the different types of social interactions they engage in on the other. Due 
to the limited number of social interaction types, this study entailed a highly skewed distribution of actors, with a large number of actors and a small number of social interaction types. Another limitation is the fact that for bipartite networks, connections should be binary variables. In the case of this research, a substantial difference exists in the intensity of individual activity. Some people created 200 posts, while others created only one. By making these variables binary, the results would be highly skewed. Therefore, we decided to weight the scores and split networks into smaller sets to have three representative networks with comparable samples and one complete network. 



\section{PART V:}

CONCLUSIONS \&

DISCUSSION
Part $V$ symbolizes the final part of this dissertation. Here we make some overarching conclusions. Give some limitations and directions for future research, discuss the main contributions of this research on a theoretical level and a practical level. and end with a brief discussion related to the research project and its results. 



\section{2 \\ Conclusions and Discussion}

\section{I2.I. Conclusions}

Realizing a thriving CoP is the goal that an increasing number of organisations have set themselves when they express the ambition to optimize knowledge management, cooperation and social interaction between employees. Organisations want to capitalize on the enormous potential that often exists among their employees to optimize the efficiency, effectiveness and innovativeness of the organisation. The purpose of this research was to create a better understanding of community developments and to support those developments. For this dissertation, the following definition of a CoP has been formulated: a social network where people in an organisational context come together around a common topic, passion or interest and regularly interact on- and offline with a focus on knowledge management, innovation, learning and social networking. Common objectives that organisations have when introducing a CoP include stimulating collective learning, innovating and improving practices, making knowledge management more efficient, creating tangible, measurable and value-adding benefits for the organisation, and increasing opportunities for professional networking. A good understanding of the objectives that organisations have for CoPs seems crucial for monitoring and evaluating developments within the CoPs (Chapter 2).

A general acceptance and adoption of CoPs is not yet widespread in organisations. Wenger \& Snyder (2000) pose three explanations for this, and with a little adaption, these explanations still stand: (I) organisations do not use the term 'community of practice' when speaking about their initiative(s), (2) only the forward-thinking organisation has fully adopted CoPs, and (3) fundamentally, there is a lack of expertise for building and maintaining CoPs and/or to integrating them with the rest of an organisation. This exploratory study focused on this last explanation and aimed to provide insight into how and why knowledge management and strategies for the continuing professional development of individuals and groups in CoPs develop, all of which is included in the following general research question: How and why do communities of practice in organisations develop? This study aimed to identify those factors that enable the development of a thriving CoP. Insight into these factors enables us to stimulate or manipulate these developments. The focus has been on evaluating and monitoring the developments within CoPs. To create an accurate picture, we developed an extended version of the motivation, opportunity, and ability model (MOA model) with a fourth construct: communality. The subsequent framework (the MOAC framework) formed the basis for this dissertation. The studies were categorized with three goals in mind: (I) studying factors that affect individual social behaviour in CoPs, (2) understanding group processes in CoPs, and (3) developing a community evaluation and monitoring methodology. Modelling behaviour in CoPs and developing this methodology was expected to contribute to expertise in building and maintaining a CoP. 


\section{I2. I. I. Individual Social Behaviour in Communities of Practice}

The first research question primarily focuses on an individual's social behaviour and follows a traditional socio-psychological approach to determine what factors affect this behaviour in CoPs. The research question is as follows:

What individual, organisational and community factors affect the social behaviour of community members in a community of practice?

The idealistic image that organisations have of the developments in CoPs often does not reflect reality. Community members see-as much of the literature already indicated-a CoP more as a source of knowledge and information and less as a place to stimulate the joint development of practices. This is seen as the result of the role of computer-mediated communication in society. It is much easier to join online communities than to join conventional CoPs, and the membership criteria for becoming a member of an online community are less strict. According to Zhang and Watts (20I5), it is often easy to become a community member if one is connected to the digital infrastructure where the community meets. This also has consequences for the intensity of the social behaviour. The fact that individuals can increasingly watch social interactions in an anonymous way also results in a rather substantial group of knowledgeacquiring community members. However, the fact that-even with the relatively large group of light users of online community software-it remains clear that the collective contributions from all community members can, despite the minimal individual contributions, still have a significant effect. Aside from a wide variety of other factors that can influence individual social behaviour in CoPs, chapters 6,7 and 8 focused on a set of potentially important factors brought together in the MOAC model, which was defined in Chapter 3 and tested in four cases which are described in Chapter 4. These factors are categorized along three dimensions: individual factors, organisational factors and community factors.

In Chapter 6, the focus was primarily on the individual factors that are related to an individual's social behaviour in a CoP. Many community members who primarily search for knowledge within a CoP feel social pressure from the organisation (management and/or colleagues) to at least be a visible member, which is often realized by registering for the CoP.This is because there is a small, negative relationship between the presence of subjective norms (e.g., social pressure) and the extent to which individuals consume knowledge and information. Thus, the members who experience more social pressure are less inclined to consume knowledge and information and vice versa. This negative relationship is also noticeable in the social interaction behaviour but is weaker and not significant. The motivation to improve the social reputation of individual members, on the other hand, contributes to social interaction behaviour within the CoP. Participants who want to profile themselves within the CoP also show this through more active types of social behaviour. The social interaction behaviour in a CoP is largely affected by the motivation to improve one's work performance, while the level of knowledge acquisition behaviour is predominantly affected by the motivation to be informed and to improve work performance. Regarding the individual's ability to participate in a meaningful way, we can conclude that this is positively related to any type of social behaviour. Speaking about an individual's ability to make meaningful contributions to a CoP is speaking about that individual's self-perceived ability to gain knowledge-operationalized as self-efficacy — to share knowledge and to create social connections with other community members. In short, the participants all feel that they have the capacity to make a meaningful contribution to the CoP. An individual's ability in this dissertation is used synonymously with the concept of self-efficacy, which is one's 
belief in one's ability to "execute courses of action required to deal with prospective situations" (Bandura, 1982, p. I22). The results in this chapter and in the chapters 7 and 8 are controlled for organisation and gender.The results show strong relationships between the organisation and an individual's social behaviour, which implies that - as may be assumed within CoPs - the variance is influenced by the organisations participating in the CoPs.

From an organisational perspective, it seems to be important to let employees perform optimally within the CoPs. The role of the organisation is to stimulate individual and group behaviour in the CoP. It is very important that the preconditions for participating in the CoP are optimally fulfilled. In this section, we report the important conclusions that lie within the sphere of influence of the organisation. The organisation has a substantial impact on the success of a CoP. In Chapter 7, the individual perceptions of the extent to which an organisation facilitates social activity in the CoP were examined. The results show that the level of individual work autonomy is rather high for those who participate in CoPs and show a positive significant relationship with the knowledge acquiring type of social behaviour in the CoPs. However, the intensity of social behaviour is rather low, which can indicate that organisations provide community members with enough freedom to perform in the way they want, but this freedom does not result in more social activity. The organisational support operationalized in technological and substantive support was also positively and significantly related to the types and intensity of social behaviours.

Specific community-related factors that might affect an individual's social behaviour in a CoP are defined in Chapter 8. This chapter focused on individuals' perceptions of communality in the CoPs. Three factors were the primary focus in this chapter: shared identity, shared leadership, and shared language. It was an assumption that these three factors formed an important indicator for the level of communality in CoPs. The results show that shared identity and shared leadership contribute to the social behaviour of community members in a CoP.The larger the shared identity and level of shared leadership in a CoP were, the higher the social behaviour. In short, speaking generally about the individual social behaviour of community members in CoPs, we can conclude that the behaviour is mainly affected by the motivation to be informed, the individual's ability operationalized as self-efficacy, the reported facilitating conditions, and the shared identity that is experienced within the CoP.

\section{I.2. Group Behaviour in Communities of Practice}

In this part of the conclusion, the emphasis is on improving our knowledge and understanding of developments in group behaviour within CoPs. The research question formulated for this is as follows:

How does group behaviour develop in a community of practice?

To answer this research question, we look at three perspectives to evaluate group behaviour. Community development patterns, the network positions of individuals based on their behaviour in the group, and the role of social interaction patterns based on bipartite network graphs. In the three chapters, the group behaviour and the role of the individual in this behaviour are central. More insight into group behaviour ensures that developments within the CoPs can be better interpreted.

First, we determine the community development patterns in CoPs. The analysis of web-based 
social interaction data in Chapter 9 uncovered remarkable community development patterns. Based on the community development patterns we found, we can conclude that in most cases, the norms for a successful CoP-which, in this dissertation, stands for a highly interactive group of participants who want to advance organisational practices by means of a variety of types of social interaction-are not met in the starting phase of a CoP. Most CoPs show a clear start-up phase in which the first primary goal seems to be to optimize knowledge management exemplified in a knowledge-centred pattern. In only two cases-the youth care institution and an in- and externally focused CoP focused on social care - the starting phase of the CoP, defined as the first fourteen months of a CoP, lead to an interactive community in which the focus was not primarily on one of the types of social interaction (e.g., creating a post, commenting on a post) visualized in a community-centred pattern. Considering the general community development patterns in all of the CoPs show a turbulent first five months with strong fluctuations in the various types of social interaction, a development that has been recognized by McDermott (2000). Without intensive support from a community management group, CoPs then lose attention within the foreseeable future, which means that social activities steadily decline.

In the final chapters (both chapters 10 and II), various datasets were linked in order to look at the possibility of gaining better insight into the group processes within CoPs. The four organisations and their employees who gave permission to merge their answers from a survey with their web-based social interaction data from the online platform were evaluated to identify remarkable patterns and test for possible explanations based on the survey data. This approach ensured that the one-dimensional evaluation by means of a survey was enriched with the time factor by examining all social activities that took place over a certain timeframe within a CoP. Using the perspective of individual community network positions and the motivating factors related to these positions yields remarkable results (Chapter 10), one of which is the relationship between social network positions and an individual's reported opportunity and perceived ability. People who have a more central position in a CoP are more positive about the opportunities they get from-for example-their organisation. The people with a more significant position in the CoP also feel that they are perfectly able to contribute to the CoP in a meaningful manner. These perceptions of individuals regarding the factors in the MOAC framework and their actual network position show a strongly polarizing development. The more centrally positioned an individual is within a CoP, the greater that person's sense of individual ability and perception of the opportunities offered. The less centrally positioned people are less likely to feel that they have the capacity to make a meaningful contribution to the CoP and feel less strongly that the organisation creates optimal conditions - a development that is increasingly characterizing online communities (Bell, 2007).

Focusing on the fluctuations and patterns in the development of CoPs, we can conclude that an ever-increasing polarization within the CoPs may indicate a power law distribution. Power law-also referred to as the Matthew Effect (Stephens et al., 2016)-refers to an active group of participants that become increasingly active compared to the less active participants. A further investigation of the social interaction patterns results in the insight that community members' social interaction behaviour is highly centralized around a small group of people (Chapter II). This is shown in the social interaction patterns of activity spread and shared affiliations. The spread of activity indicates that community members' active behaviour is highly centralized around a small number of people. The degree of shared affiliation indicates that community members who share one type of social interaction in a CoP are more likely to start other types of social interactions. While considering the MOAC factors and their relationship to both the 
social interaction patterns and an individual's network position, we merged the data files (the survey and web-based interaction data). Generally, we can conclude that the level of motivation, one's ability to perform and the opportunities that individuals receive from their organisation positively contribute to community members who initiate similar types of social interactions in a CoP. A more imaginative interpretation of this result is that people with a higher level of motivation often exhibit similar behaviours.

The common denominator in these three studies is the use of a complementary set of methods and techniques to evaluate group behaviour based on web-based social interaction data using a pattern recognition approach. The results show that group behaviour within CoPs largely revolves around the contributions of a small group of participants who are responsible for the lion's share of social interactions. The result is that the often-stated objective of reducing the social distance between employees - which with the ambition to reduce barriers between departments also applies to the four cases in this dissertation-does not yet seem to have been achieved by the development of these CoPs (four cases). In contrast, the social distance between the participants seems to be increasing, given the strong relationship between the level of degree centrality in a CoP and the factors of opportunity and ability. The social interaction patterns also show a similar result, given that community members with a higher level of motivation, degree of reported opportunities, and perceived ability are more likely to reciprocate their type of social interaction with other community members. Depending on the group of active contributors, the group behaviour develops in a certain direction, which is usually a knowledge-centred direction or, in two case, a community-centred direction. In any case, the form of polarization that can be identified within the group behaviour is an important indicator of the value given to the CoP by the participants.

In short, speaking about group behaviour in CoPs is speaking about predominantly knowledgecentred developments, with a special role for a few centrally located people and in which the social distance between the participants is increasing rather than decreasing.This is also reflected in the social interaction patterns through the degree of shared affiliation, where community members often co-initiate similar behaviour. In addition, the degree of motivation, the reported opportunities offered by the organisation, and the perceived ability (self-efficacy) are strongly related to showing comparable types of social behaviour. Thus, the higher the scores on these factors, the more likely it is that people will show similar types of social behaviour.

\section{I2. I.3. Community monitoring and evaluation methodology}

The primary purpose in this dissertation was to generate an improved understanding of developments in CoPs and expertise for building and maintaining CoP. To not only increase the understanding of these developments within CoPs but also to monitor and evaluate them in a more continuous manner, this dissertation has taken the first step taken towards the development of a community monitoring and evaluation methodology. Together, the methods and techniques used in this dissertation form a first step towards a community monitoring and evaluation methodology (CMEM). CMEM is a multi-method approach that combines subjective data with objective data in a predominantly data-driven manner.The research question that was formulated for this purpose is as follows:

What is a relevant methodology for the monitoring and evaluation of individual and group behaviour in communities of practice? 
The developed CMEM combines various research disciplines, methods and techniques to establish a meaningful approach for evaluating and monitoring developments within CoPs. The methodology is a multi-method approach that uses subjective data alongside objective data, uses large existing datasets that are approached with data-driven principles, and applies a complementary set of methods and techniques that provide (new) insights into the developments in CoPs (Chapter 5 and in various parts of the empirical studies).

By taking advantage of the increases in technology use, it is possible to enrich subjective data collected directly from study participants with objective data that can be obtained from their actual use of these technologies. In this dissertation, the integration of methods and techniques made meaningful use of existing data sets like those that are collected and stored within most online community software packages, and this use maximizes the insights offered here. Browsing the related databases creates opportunities to recognize interaction patterns and networks that provide insight into the behaviour of community members and the developments in this behaviour. Looking purely at this web-based social interaction data offers the opportunity to describe what was plainly evident and omit the truly complex subjective data (Morse, 20I5). This new opportunity supports us in the design of CMEM by acquiring insights from descriptive, predictive and even prescriptive community analytics, but it lacks explanatory power for these interaction patterns and networks - a situation that occurs in most studies using webbased social interaction data (Mccully \& Lampe, 20I I; Saarinen et al., 20I5). Oftentimes, these studies will use mixed-method approaches, whereby web-based social interaction data will be supplemented with, for example, subjective data. For this methodology, it was decided to include a questionnaire to determine the perceptions of individuals and to relate them to web-based social interaction data. By developing a questionnaire that also includes specific community factors (communality), an extensive version of the motivation, opportunity, and ability model, the MOAC model, was developed. The factors from this MOAC model are related to behaviour and interaction patterns in order to gain more insight into the creation and development of CoPs and the social behaviour in it. The composition of this hybrid model is based on the MOA model, the theory of planned behaviour (TPB), the technology acceptance model (TAM), social identity theory, and social capital theory. We followed the advice of Hughes (2007) in blending aspects of other theories with construct relationships borrowed from the MOA model. Therefore, the MOAC model is not the basis for this research but rather the main hook to which various explanatory variables can be connected. The original MOA model merges the explanatory variables for individual behaviour with both organisation-specific variables and variables that influence individual capacity in one model.

The combination of subjective (questionnaire) with objective (web-based social interaction) data enables the interpretation of patterns and developments that are useful for both the monitoring and the evaluation of CoP developments. This combination provides opportunities to "make sense of complex phenomena, to dissect and/or to synthesize, to abstract, to theorize, and to recognize how this fits into the work of others, all of which enables generalization and application" (Morse, 20 15, p.587). In addition, the combination enables us to provide a better, more complete picture of the developments that occur in real-life settings. This dissertation used a multi-method, hybrid study design with separate but coherent studies, and techniques that were appropriate for the data sets and that result in an improved quality of the findings. In particular, the methods used to handle large data sets contribute to our understanding of developments in CoPs and allow us to compare various CoPs regardless of their size. The increasing number of methods that contribute to the ambition to make more meaningful statements about large data sets is growing each year.The resulting data structures that become 
available through, for example, digital technologies often differ from typical—traditional—studies, thus requiring methodological innovation (Mahrt \& Scharkow, 20I3). One of the drawbacks of using data from digital technologies is that tried and tested methods and standards of social science research are not always compatible with the data formats and structures for digital media research. The structure of the data storage often changes over time-for example, due to the efficiency and effectiveness of data processing - which can ultimately lead to structured data suddenly becoming unstructured. However, it remains important-as already stated by Erhan et al. (2019) — to deal with issues of data quality in objective data such as uncertainty (e.g., content, reading time), inconsistency and redundancy. Identifying and interpreting interaction patterns with the reality and background of the cases in mind is therefore crucial to prevent incorrect analyses as far as possible.

To answer to the third research question, we developed the community monitoring and evaluation methodology (CMEM) that uses different datasets to allow an analysis of the behaviour of individuals within CoPs from different perspectives. In this dissertation, individual behaviour and group behaviour were analysed by using a questionnaire and merging it with web-based social interaction data. The result is a methodology that provides adequate insight into the developments within CoPs and, by using the questionnaire, also provides possible explanations for these developments.

This dissertation has yielded several interesting results with the identification of factors that affect certain social behaviour within communities of practice, with an improved insight into developments in group behaviour, but certainly also with the development of a methodology for monitoring and evaluating community development. Knowledge and understanding that form a valuable contribution to both science and practice.

\subsection{Limitations and directions for future research}

In the individual chapters, we have already focused on the various limitations of the associated studies. In this section, we discuss the overarching limitations and give directions for future research. In the section that follows, we focus on the theoretical implications of the findings in this dissertation. The practical implications of the results will be discussed in the penultimate section, after which we end with a discussion. In this chapter, we describe five of this work's overarching limitations and possibilities for future research. As limitations are an opportunity for further research, we would highly recommend considering the limitations here as such. In general, these limitations and directions for future research can be classified at three levels: the theoretical level, methodological level and practical level.

First, on the theoretical level, a study of the factors that affect an individual's behaviour in CoPs can be conducted from various perspectives and with multiple theories in mind. Research can, for example, focus primarily on one of the factors from the MOAC model or from comparable theories such as the Theory of Planned Behaviour (TPB), Theory of Reasoned Action (TRA) or TechnologyAcceptance Model (TAM) and can also focus on specific personality traits, participants or organisational characteristics or on social capital theory or social identity theory. Given the exploratory nature of this research project, it was decided to include a significant number of indicators that might influence the behaviour of individuals in social environments. Theories such as TPB, TRA and TAM are rather narrow in nature, which makes them less suitable for the exploratory context of this dissertation. This, in combination with the consideration that the questionnaire should not be too long, led us to include a minimal number of items to cover a 
few variables. For example, the variables of social reputation, subjective norms, and facilitating conditions are measured with one or two indicators. Another extreme is the variable of work performance. This variable was initially split into two smaller units: work efficiency and work quality. However, since these variables are highly correlated with each other, we decided to merge them into one variable. For future research, at least two research directions are possible with regard to the further elaboration of the MOAC model: (I) a specific focus on components of the MOAC model, primarily, for example, the motivation, opportunity, ability or communality or (2) explanatory and/or confirmatory research to further test the MOAC model as a theory.

Second, we address limitations of and directions for future research at the methodological basis of this research. In Chapters 6, 7 and 8, a cross-sectional survey was used in which data were collected at one time point. In Chapters 10 and II, the survey data were connected to the webbased social interaction data, which were longitudinal in nature. Therefore, the chance that the motivations, reported opportunities, perceived abilities and/or perceived communality changed during the process of the CoP's maturation was likely. A longitudinal survey to compare the results would therefore offer an adequate opportunity to control for these changes but also to provide insight into differences and developments. Another option to improve the explanatory power of the methods is to periodically test components of the MOAC model in the CoPs by means of short, easy-to-complete questionnaires integrated directly into the software of the CoP. This increases the efficiency and cost-effectiveness of collecting, storing and converting suitable data sets and involves larger datasets with more statistically robust findings (Erhan et al., 2019).

Another methodological limitation is the fact that this research was conducted in the Netherlands with only Dutch respondents. To enable others to use the questionnaire in other countries and cultures, we have translated the questionnaire as accurately as possible into English. The fact that the research was conducted in the Netherlands also reduces the external generalizability of the results to other countries and cultures. Other countries are not included in the measurements, so different cultural aspects can affect the developments within a CoP.

An interesting limitation relates to the phenomena of group polarization. When analysing the developments in the CoPs, we discovered a pattern that looked suspiciously like group polarization. Group polarization is the result of increasingly homogenous group dynamics. Central community members become increasingly central, which consequently means that fewer central community members are becoming less central. In addition, the central community members also perceive an increasing amount of organisational support (opportunity) and improved skills and competencies (ability). This form of group polarization is purely based on factual social behaviour, but since the substantive conversations are missing, it is difficult to determine the reason for this. For future research, it is advisable to include qualitative methods (e.g., content analysis) in addition to quantitative research methods to create an even better understanding of the developments.

A last methodological limitation is the absence of sequential analytics. The procedure of the online community software provider was to store all types of social interactions in separate tables, with references between the 'post table' and the 'comment-table'. Therefore, we have been able to establish a relationship between a given post and a given comment, but not between two comments. Literally, some comments referred to previous comments, but technically, a link was made between a comment and a post. Insights into threads-operational in sequential analytics-enable researchers to gain a better understanding of the influence that certain CoP 
members can have on the CoP's development. For example, the comment of one person might have more influence on the development than would the comment of another person.

The third level is the practical level, with specific emphasis on a direction for future research. The CMEM developed in this dissertation is currently a conceptual methodology tested in several cases. It is recommended to set a next step to an actual visualization of the CMEM as monitoring and evaluation dashboards and testing the applicability and usefulness for the stakeholders. It is expected that the technical processing of the CMEM into practically usable dashboards will contribute to enabling an organisation's management, community managers and community members to make statements in a meaningful way but also to make directed interventions regarding community development activities.

\subsection{Theoretical implications}

After considering the different outcomes of the studies described in each individual chapter, we come to the following theoretical implications that contribute to our understanding of the monitoring and evaluation of CoPs in work contexts. In general, this dissertation contributes to theory development in three ways: (I) the multi-level application of the MOAC model, (2) the extension of the MOA model with the communality factor, and (3) the integration of complementary methods and techniques for the evaluation and monitoring of developments in CoPs that use new forms of data acquisition.

First, the original MOA model is mainly focused on individual social behaviour; in this research, it has been divided into three levels, namely, individual, organisational and community. The result is the motivation, opportunity, ability and community model (MOAC model). The results show that this model is, to a large extent, suitable as an overarching model for evaluating social behaviour within CoPs and provide with expertise for building and maintaining CoPs. Since the MOAC factors are more extensively operationalized than they are in the original MOA model, we can explain social behaviour more thoroughly. Hughes (2007) suggested using the MOA model as a hybrid theory that blends aspects of other theories. With the MOAC model, the hybrid models in which the MOA model is an important component have been taken a step further.

Second, adding communality to the MOA model has been done with the assumption that this factor is an important indicator of social behaviour in a CoP. The opportunity in its definition reflects the context supported by the organisation. However, the CoP also produces its own identity and has its own unique atmosphere, which is expected to have a major impact on individual behaviour in CoPs. This is the main reason that the factor of communality was added to the MOA model. Initially, the MOA model was designed to provide insight into the influencing factors for social behaviour, which would involve explaining behaviour and also sketching out a framework or context. The opportunity factor plays an important role in this process. However, the opportunity factor primarily focuses on the influence of organisations, while CoPs also produce their own unique atmospheres that are presumed to have a large influence on individual behaviour in CoPs. That is the main reason that the factor of communality was added to the MOA framework to measure whether the existence of a sense of community within or between organisations is present. In this thesis, a 'sense of community' is interpreted as a concept that is similar to 'communality'. The studies in this dissertation have shown that the underlying variables (shared identity and shared leadership) used to measure communality at the community level also affect social behaviour on its own to a varying extent. Further, the reported communality has been tested by relating it to network positions. The results 
indicate that the level of degree centrality becomes more closely related to the perception of communality as the development of the CoP progresses.

Third, the integration of methods and techniques used in this dissertation to monitor and evaluate community developments from various perspectives is unique in nature and forms a promising start for more research into community developments in which the role of existing datasets is enriched with additional datasets. The methodology describes developments within CoPs from the perspective of community development patterns, network positions, and social interaction patterns. This provides insight into the influence of individual behaviour on group behaviour but also into group behaviour in general. In addition to these implications, this approach enables other researchers to investigate actual social group behaviour in other more objective and valid ways, as web-based social interaction data provide a more accurate reflection of social behaviour compared to most other forms of data collection (Scharkow, 2016). This feeds a strongly emerging inter- and cross-disciplinary scientific field: data science (Cao, 2017).

Given these three theoretical implications we contributed to the development of expertise by understanding the community developments by applying the MOAC model in a multi-level manner, by further expanding it with the contextual factor communality, and by integrating complementary - increasingly data-driven - methods and techniques suitable for the evaluation and monitoring of developments in CoPs that use new forms of data acquisition.

\subsection{Practical implications}

Turning to the practical implications, the findings of this research contribute to practice by guiding the monitoring and evaluation of developments in CoPs. One of the discussions in the literature is whether CoPs can be created and managed by organisational management. In this thesis, we did not address this issue specifically but have delved into various parts of community development where the role of the organisation should not be underestimated. This section deals with the practical implications at three levels: (I) organisational management, (2) community management, and (3) online community providers.

\subsection{Implications for organisational management}

CoPs were initially introduced in organisations to reduce the social distance between employees, as stated in the case description in Chapter 4. Technological developments have made it easier to take this step to reduce such distance.All of one's colleagues can be approached at the touch of a button. However, an analysis of group behaviour shows increasing polarization within the CoP networks. It seems that the more centrally positioned community members experience more opportunities from the organisation and experience an increased individual capacity. Given the data that this polarization demonstrates, developments within CoPs seem to indicate an increasing social distance between employees. Organisations can improve the opportunities and individual capacity of the other community members through improved training and, in doing so, can respond more effectively to work autonomy needs-for example, by clarifying the relevance of the CoP at both the individual and the community level.

\subsubsection{Implications for community management}

First, implicit and explicit goals and objectives set by organisations do not seem to be achieved fully or at all in the first fourteen months of the CoP.Within the four cases in this dissertation, 
explicit objectives were set prior to the implementation of the CoPs. The inclusion of these objectives in a community management plan and compliance with these objectives can offer the possibility to contribute to meaningful interventions in certain developments. The results from the studies show that most CoPs show predominantly knowledge-centred developments, which implies that just one objective is being met: improving knowledge management. Another objective-improving collective learning-is, in most cases, realized due to the large group of knowledge absorbers. The objective of improving professional networking activities is often not met, as the primary type of social interaction focuses on knowledge sharing and knowledge consumption. Another, more implicit objective that many organisations have set themselves is to increase the social interactions between employees both within and between departments. This objective does not seem to have been achieved because most CoPs focus primarily on one type of social interaction exemplified in the community development pattern: the knowledgecentred pattern. Community managers can use community-development patterns to track developments in the CoP. In addition, community managers can use insights regarding the network positions of individual community members within the CoPs to strategically monitor and steer individual behaviour within the group. This enables community managers to intervene in a more targeted way to achieve their implicit and explicit objectives. For example, if the goal is to spread knowledge through the organisation more rapidly, then identifying the bridges in the organisation is recommended. To identify the most committed community members, determining the centrally positioned community members is recommended, and to steer or stimulate discussions to some extent, it is recommended that the community leader will be identified. He/she will be able to direct social interactions in a certain direction. The peripheral group in the CoPs should be strongly involved through, for example, training sessions and personal and group discussions to determine needs that the CoP can meet.

Second, as reported in the first three empirical chapters (Chapters 6,7 and 8), the difference between the knowledge-acquisition behaviour and the social interaction behaviour are linked to different variables in the MOAC model. Practitioners must therefore consider the changing factors that influence the behaviour of different participants. A good insight into the types of users, the position of individual users in the network, the level and types of activity and the goals and objectives set with regard to the CoP is therefore crucial for the member-tailored stimulation of social behaviour. As seen in Chapter 9, different types of community members can make strong contributions towards certain developments, and in addition, the level of degree centrality (Chapter 10) is largely affected by an individual's opportunities and his or her perceived ability. By including these two factors in a community development policy, community members are more likely to show more active social behaviours. One of the typical examples of how these two variables can be integrated into a community development policy is by paying special attention to the possibilities (e.g., best practices, the results of experiments, and lessons learned) within the CoP that are unique compared to existing alternatives.

Third, it has been found that the level of social behaviour in the CoPs was predominantly low. The objectives set by the organisations, such as cross-departmental cooperation, were not put into practice in many organisations. Participants in the CoPs mainly participated to be informed-a finding that corresponds to the behaviour in almost all public and private social media in which the vast majority absorbs information instead of sharing knowledge. However, in some CoPs, there are promising signs of actual community-developing activities. Regarding the motivation to improve work performance, a significant link is found with the more active type of social behaviour in a CoP_-social interaction behaviour-and not with knowledgeacquisition behaviour. This might imply that community members willing to improve their work 
performance feel more connected to the CoP. For community management, this is an indication that adequate interventions are needed to show the added value of the CoP regarding work performance. One way to realize this is by creating a content strategy based on storytelling with typical examples of how the CoP has contributed to the more efficient or effective execution of existing work activities.

Fourth, the results from the different studies also substantially contribute to the community management strategies of community managers. Making recommendations for practice ultimately remains somewhat context-sensitive, since what applies to one party not automatically applies to another party. Nevertheless, looking at recurring patterns, we can conclude that some patterns occur in the majority of communities. For example, taking the results in Chapter 9 into consideration, one recurring social behaviour pattern is that almost every CoP fluctuates in the start-up phase of the community's implementation.A CoP often makes a great start, after which the enthusiasm of some of the community members slowly fades away. Community managers who directly implement the CoP by including the entire organisation should, in that case, immediately look for the possible cause of this decrease in enthusiasm. Through an adequate response from the community manager to-in this case-a diminishing enthusiasm among (a few) community members, the possibility arises of quickly repairing the damage suffered. The community development patterns act as a warning system for community managers to keep an eye on the developments.

\subsubsection{Implications for online community providers}

As stated in the introduction of this dissertation and later in each chapter, it was our ambition to create a relevant methodology for the monitoring and evaluation of individual and group behaviour in CoPs. Particularly in the last three chapters of this dissertation-Chapters 9, 10 and II-special attention was given to web-based social interaction data already available to most of the community managers. As stated in the previous paragraph, a multi-method, hybrid methodology has been developed that incorporates both subjective and objective data. This methodology offers extensive opportunities to approach CoPs from a variety of perspectives. Looking at community development patterns and the key positions in a CoP and analysing specific social interaction patterns ensures an increasingly precise view of the background of community development. The adequate use of these insights and the enrichment of the 'hard' behavioural data with the 'soft' social data contributes to better community management policies and strategies.

An initial suggestion is to incorporate the methods and techniques used in this dissertation into well-structured and actionable dashboards to provide community managers with guidance for monitoring and evaluating the developments in their CoPs and intervening where possible or necessary. An example of such a dashboard might be a web page or application that is linked to the database of the online community software and shows visualizations based on the continuous stream of new web-based social interaction data combined with unique data from each individual community member (e.g., age, gender, occupation, polls). One of the dashboards could, for example, show a social network graph that displays the relevant people in the community based on their network positions. Since historical data are also available, it is also possible to gain insight into the historical development of individual network positions during the period at the start of the CoP.

\subsection{Discussion}


To conclude this dissertation, three statements for discussions will be made. As stated at the beginning of this dissertation, CoPs are seen by organisations as an important method for developing a knowledge-driven strategy with individual continuous professionalization and social learning. Reflecting on the results of this research, we argue that organisations can benefit much more from these CoPs, especially considering the increasing technological possibilities. Technical possibilities make the development of CoPs in online environments an increasingly attractive method for optimizing knowledge management, collaborating and social networking. A growing number of organisations are therefore deciding to purchase online community software in order to offer employees the opportunity to improve their individual and collective knowledge levels, to stimulate collaboration and to start social networking activities. As a result, it seems that organisations chose a CoP and the underlying technology mainly from an ideological point of view in order to create a networked or learning organisation and less as a way of working to achieve goals in which the working method behind a CoP can be of particular value: for example, creating a common identity, improving solidarity, and reducing the social distance between employees. However, whether a CoP will be of value depends on the organisational goals. The results show that goals are often set implicitly in order to, for example, improve the exchange of knowledge but that no explicit goals are set that are also provided with key performance indicators.

Reflecting on the developments taking place within the CoPs, we can state that they are often still at a stage of exploration and experimentation. Since the introduction of the concept of a 'community of practice' (Lave \& Wenger, 1991), many organisations have started initiating CoPs with varying degrees of success (Chua, 2006). The results from this study show that the CoPs where the focus is primarily on increasing the number of online social interactions are, in particular, developing more poorly. Within these CoPs, there is more often a fluctuation in social activities. Where a clear goal and vision seem to have been chosen, the development is much more stable. However, in general, most CoPs in this study seem to be less in line with what is normatively defined as a CoP. Most CoPs in this thesis show fluctuations that are too strong to speak of the CoP as having a stable development. This finding brings us to the proposition that when CoPs are fully supported by the management layers within the organisation, when the integration into existing organisational policies is improved, and when goals and objectives are clearly formulated, evaluated and monitored, the CoPs should be better able to grow from the more conceptual or experimental phase to a more advanced phase.

The development of a community monitoring and evaluation methodology (CMEM) is an important step towards making meaningful use of structured and unstructured datasets. However, there is still more work to be done. This mainly concerns the size of the datasets which improves the generalizability of the results and the methodological possibilities for dealing with the various data sources. Further expansion of the CMEM may move in the direction of text mining, which would enable us to answer questions such as "What are relevant message types that affect community development patterns?" and "Which individual communication styles are strongly related to social interaction patterns in CoPs?"

This research has contributed to our expertise in building and maintaining CoPs and our understanding of the how and why of developments in these CoPs. Our CMEM contributes with an approach for actual and data-driven monitoring and evaluation of CoPs in organisations. Based on these contributions, we go beyond the hype about CoPs in organisations and take a new step towards a more targeted development of CoPs. 



\section{References}

Abraham, Ajith, A.-E. H. \& V. S. (2010). Computational Social Network Analysis. Statewide Agricultural Land Use Baseline 20 I5. London: Springer-Verlag. doi: I0. I0 I7/CBO978I I074I 5324.004

Adler, P. S., \& Kwon, S. (2002). Social Capital: Prospects for a New Concept. Academy of Management Review, 27(I), I7-40. doi: I0.5465/AMR.2002.59223 I4

Agneessens, F., \& Roose, H. (2008). Local Structural Properties and Attribute Characteristics in 2-mode Networks: $p$ * Models to Map Choices of Theater Events. The Journal of Mathematical Sociology, 32(3), 204-237, doi: I0.1080/00222500802 I 48685

Ajzen, I. ( 1985). From intentions to actions:A theory of planned behavior. In Springer-Verlag (Ed.), Action control: From cognition to behavior. Berlin, Heidelberg, New York.

Ajzen, I. (199I). The theory of planned behavior. Organization Behavior and Human Decision Processes, 50(2), I79-2II. doi: I0.1016/0749-5978(9I)90020-T

Ajzen, I., \& Albarracín, D. (2007). Predicting and Changing Behavior:A Reasoned Action Approach. In Prediction and Change of Health Behavior.Applying the Reasoned Action Approach. London: Lawrence Erlbaum Associates Publishers.

Alavi, M., Kayworth, T. R., \& Leidner, D. E. (2004).An Empirical Examination of the Influence of Organizational Culture on Knowledge Management Practices. Journal of Management Information Systems, 22(3), I9I-224. doi: I0.2753/MIS0742-I 222220307

Andersson, T., Formica, P., \& Curley, M. G. (2009). Knowledge-driven entrepreneurship: the key to social and economic transformation. Springer Science \& Business Media.

Andrews, D. C. (2002). Audience-Specific Online Community Design. Communications of the ACM, 45(4), 64-68. doi: I0.I I 45/505248.505275

Andriessen, J. H. (2005). Archetypes of knowledge communities. Proceedings of the Second Communities and Technologies Conference, Milano 2005, I9|-2 I3. doi: I0. 1007/I-4020-359 I8-II

Ang, L. (20I I). Community relationship management and social media. Journal of Database Marketing and Customer Strategy Management, I8(I), 3 I-38. doi: I0.1057/dbm.20 I I.3

Annabi, H., Mcgann, S. T., Pels, S., \& Arnold, P. (20I2). Guidelines to Align Communities of Practice with Business Objectives:An Application of Social Media. Proceedings of the 45th Hawaii International Conference on System Sciences, 3869-3878. doi: I0.I I09/HICSS.20 I 2.297 
Ardichvili, A. (2008). Learning and Knowledge Sharing in Virtual Communities of Practice: Motivators, Barriers, and Enablers. Advances in Developing Human Resources, 10(4), 54 I-554. doi: $10.1177 / 1523422308319536$

Ardichvili,A., Page,V., \& Wentling, T. (2003). Motivation and barriers to participation in virtual knowledge-sharing communities of practice. Journal of Knowledge Management, 7(1), 64-77. doi: |0.| | 08//36732703/0463626

Argote, L. (20I2). Organizational learning: Creating, retaining and transferring knowledge. Springer Science \& Business Media.

Asur, S., Parthasarathy, S., \& Ucar, D. (20I5). An Event-based Framework for Characterizing the Evolutionary Behavior of Interaction Graphs. ACM Transactions on Computational Logic, 2(3), I-35. doi: I0.I|45/I28 I | 92.I28I290

Atzmueller, M. (20I4). Data Mining on Social Interaction Networks. Journal of Data Mining \& Digital Humanities, 20 I4. Retrieved from https://arxiv.org/pdf/I 3 I 2.6675.pdf;

Backstrom, L., Huttenlocher, D., \& Kleinberg, J. (2006). Group Formation in Large Social Networks: Membership, Growth, and Evolution. In Proceedings of the 12th ACM SIGKDD international conference on Knowledge discovery and data mining, 44-54). doi: I0.1|45/II50402.1I504I2

Bagozzi, R. P., Dholakia, U. M., \& Pearo, L. R. K. (2007). Antecedents and consequences of online social interactions. Media Psychology, 9(December), 77-I I4. doi: I0.1080/I52I3260709336804

Bagozzi, R. P., \& Yi, Y. ( 1988$)$. On the evaluation of structural equation models. Journal of the Academy of Marketing Science, I 6(I), 74-94. doi: I0. I007/BF02723327

Bakardjieva, M., \& Feenberg,A. (2002). Community technology and democratic rationalization. Information Society, I8(3), I8I-192. doi: I0.1080/01972240290074940

Baker,A.T., \& Beames, S. (20I6). Good CoP: what makes a community of practice successful? Journal of Learning Design, 9(I), 72. doi: 10.5204/jld.v9il.234

Bandura,A. (1977). Self-efficacy:The exercise of control. (Freeman, Ed.). New York.

Bandura,A. (1982). Self-efficacy mechanism in human agency. American Psychologist, 37(2), I22-I47. doi: I0.1037/0003-066X.37.2.122

Banerjee, A., Bandyopadhyay, T., \& Acharya, P. (20I3). Data Analytics: Hyped Up Aspirations or True Potential? Vikalpa, 38(4), I-I I. doi: I 0. I I77/0256090920 I 3040 I

Barab, S.A., MaKinster, J. G., \& Scheckler, R. (2003). Designing system dualities: Characterizing a Web-supported professional development community. Information Society, 19(3), 237-256. doi: 10.1080/01972240309466

Baran, B., \& Cagiltay, K. (20I0). Motivators and Barriers in the Development of Online 
Communities of Practice. Eurasian Journal of Educational Research, 10(39), 79-96. doi: I0.1 108/13673270310463626

Barnett, S., Jones, S.C., Caton, T., Iverson, D., Bennett, S. \& Robinson, L. (20I4) Implementing a virtual community of practice for family physician training:A mixed-methods case study. Journal of Medical Internet Research, I6(3), I-2I. doi: 10.2196/jmir.3083

Barragán-Ocaña,A., Quijano-Solís, Á.,Vega-Díaz, G., \& Sánchez-Lara, B. (20I2). Communities of practice: a focus from complex systems. International Journal of General Systems, 4 I (7), 74I-755. doi: I0.1080/0308I079.20I2.689625

Barry, M., Kuijer-Siebelink,W., Nieuwenhuis, L., \& Scherpbier-de Haan, N. (20I7). Communities of practice:A means to support occupational therapists' continuing professional development. A literature review. Australian Occupational Therapy Journal, 64(2), I85-193. doi: $10.1111 / 1440-1630.12334$

Bastian, M., Heymann, S., \& Jacomy, M. (2009). Gephi:An open source software for exploring and manipulating networks. In Third International AAAI Conference on Weblogs and Social Media, 36I-362. San Jose, CA. doi: I0.I I36/qshc.2004.0I0033

Bayton, J. (1958). Motivation, Cognition, Learning-Basic Factors in Consumer Behavior. Journal of Marketing, 22(I), 282-289. doi: 10.2307/I247/ I9

Behringer, N., \& Sassenberg, K. (20I5). Introducing social media for knowledge management: Determinants of employees' intentions to adopt new tools. Computers in Human Behavior, 48, 290-296. doi: 10.1016/j.chb.2015.01.069

Bell,V. (2007). Online information, extreme communities and internet therapy: Is the internet good for our mental health? Journal of Mental Health, I 6(4), 445-457. doi: I0.1080/0963823070|482378

Bender, S., \& Fish,A. (2009). The transfer of knowledge and the retention of expertise : the continuing need for global assignments. Journal of Knowledge Management, 4(2), I25-137. doi: |0.| | 08/|367327001037225 |

Bennet, A., \& Bennet, D. (2003). The partnership between organizational learning and knowledge management. In C.W. Holsapple (Ed.), Handbook on Knowledge Management (I st ed., pp. 439-460). Heidelberg: Springer-Verlag.

Bettiga, D., Lamberti, L., \& Noci, G. (2018). Investigating social motivations, opportunity and ability to participate in communities of virtual co-creation. International Journal of Consumer Studies, 42(I), I55-I63. doi: I0.I I I I/ijcs. 12409

Bettman, J. R. ( 1979). Memory Factors in Consumer Choice:A Review. Journal of Marketing, 43(2), 37-53. doi: $10.2307 / / 250740$

Bhattacherjee, A. (200I). Understanding Information Systems Continuance:An ExpectationConfirmation Model. MIS Quarterly, 25(3), 35 I-370. doi: I0.2307/325092 I 
Bigné, E., Ruiz, C., Andreu, L., \& Hernandez, B. (20I5).The role of social motivations, ability, and opportunity in online know-how exchanges: evidence from the airline services industry. Service Business, 9(2), 209-232. doi: 10.1007/s I I628-013-0224-8

Bishop, C. M. (2013). Pattern Recognition and Machine Learning. Journal of Chemical Information and Modeling, 53(9), I689-I699. doi: I0. I0I7/CBO978I I074I5324.004

Bista, S. K., Nepal, S., \& Paris, C. (2012). Engagement and Cooperation in Social Networks : Do Benefits and Rewards Help? Proceedings of the I I th International Conference on Trust, Security and Privacy in Computing and Communications Engagement, I405-I4 I0. doi: I0. I I09/ TrustCom.20I2.145

Bock, G.-W., Robert,W., \& Kim,Y.-G. (2005). Behavioral Intention Formation in Knowledge Sharing: Examining the Roles of Extrinsic Motivators, Social-Psychological Forces, and Organizational Climate. MIS Quarterly, 29(I), 87-I I I. doi: I0.2307/25 I48669

Bock, G., Kankanhalli,A., \& Sharma, S. (2006). Are norms enough? The role of collaborative norms in promoting organizational knowledge seeking. European Journal of Information Systems, 15(4), 357-367. doi: 10.1057/palgrave.ejis.3000630

Bock, G.W., Zmud, R.W., Kim,Y., \& Lee, J. (2005). Behavioral Intention Formation in Knowledge Sharing : Examining the Roles of Extrinsic Motivators, Social Psychological Forces, and Organisational Climate. MIS Quarterly, 29(I), 87-III. doi: I0.2307/25I48669

Bode, N.W. F., Sutton, A., Lacey, L., Fennell, J. G., \& Leonards, U. (20I7). A method for detecting characteristic patterns in social interactions with an application to handover interactions. Royal Society Open Science, 4, I-16. doi: http://dx.doi.org/I0.1098/rsos. I60694

Bonacich, P., \& Schneider, S. ( 1992 ). Communication networks and collective action. Social Networks, 9(4), 225-245. doi: 10.1016/0378-8733(87)90006-2

Borzillo, S., Aznar, S., \& Schmitt, A. (20I I).A journey through communities of practice: How and why members move from the periphery to the core. European Management Journal, 29(I), 25-42. doi: 10.1016/j.emj.2010.08.004

Bourhis, A., Dubé, L., \& Jacob, R. (2005). The Success of Virtual Communities of Practice:The Leadership Factor. Electronic Journal of Knowledge Management, 3(I), 23-34. Retrieved from file://C:/Users/vollenbroekw/Downloads/ejkm-volume3-issue I-article50.pdf

Bouwman, H., Van den Hooff, B., Van de Wijngaert, L., Van Dijk, J. (2005). Information \& Communication Technology in Organizations. London: Sage Publications.

Boxall, P., \& Purcell, J. (20I I). Strategy and Human Resource Management. Basingstok: Palgrave Macmillan.

Brandtzaeg, P. B., \& Heim, J. (20I I).A typology of social networking sites users. International Journal of Web Based Communities, 7(I), 28. doi: 10.1504/ijwbc.20I I.038I 24

Brass, D.J. \& Burkhardt, M. E. (1993). Potential Power and Power Use:An Investigation 
of Structure and Behavior. The Academy of Management Journal, 36(3), 44 I-470. doi: $10.2307 / 256588$

Brass, D. J. (1984). Being in the Right Place:A Structural Analysis of Individual Influence in an Organization. Administrative Science Quarterly, 29(4), 5 I8. doi: I0.2307/2392937

Brown, J. S., \& Duguid, P. (199I). Organizational Learning and Communities-of-Practice:Toward a Unified View of Working, Learning, and Innovation. Organization Science, 2(I), 40-57. doi: I0.1287/orsc.2.1.40

Bryant, S. L., Forte,A., \& Bruckman,A. (2005). Becoming Wikipedian: transformation of participation in a collaborative online encyclopedia. In Proceedings of the 2005 international ACM SIGGROUP conference on Supporting group work, I-I0.

Bun, E.,Vries, P. De, Kolfschoten, G., \& Veen,W. (20I I). Communities of Practice and the Challenge of Management Support. In Proceedings ofthe International Conference on Knowledge Management and Information Sharing, I86-193. doi: 10.5220/0002305801860193

Cabrera, Á., Collins, W. C., \& Salgado, J. F. (2006). Determinants of individual engagement in knowledge sharing. International Journal of Human Resource Management, I 7(2), 245-264. doi: I0.1080/09585| $905004046 \mid 4$

Cadiz, D. ., Sawyer, J. E. ., \& Griffith, T. L. . (2009). Developing and validating field measurement scales for absorptive capacity and experienced community of practice. Educational and Psychological Measurement, 69(6), I035-1058. doi: I0. I I77/00 I 3 I64409344494

Cambria, E., Rajagopal, D., Olsher, D., \& Das, D. (20I4). Big Social Data Analysis. In Big Data Computing, (40I-4I4). Taylor \& Francis Group.

Campo, M., \& Chaudhury, H. (20I2). Informal social interaction among residents with dementia in special care units: Exploring the role of the physical and social environments. Dementia, I I(3), 40 I-423. doi: I0. I I77/I47I30I2II42II89

Canzano, D. (2004). Knowledge Management and Collaborations : Knowledge. In Proceedings of I-KNOW'04, June 30 - July 2, 2004, 175-183). Graz, Austria.

Cao, L. (2017). Data Science: Challenges and Directions. Communications of the ACM, 60(8), 59-68. doi: 10.1 |45/3015456

Cardona, P., Lawrence, B. S., \& Bentler, P. M. (2004). The Influence of Social and Work Exchange Relationships on Organizational Citizenship Behavior. Group \& Organization Management, 23(2), 219-247. doi: I0.I I77//05960 I I0325740 I

Castillo De Mesa, J., Gómez Jacinto, L., López Peláez,A., \& Palma García, M. (2019). Building relationships on social networking sites from a social work approach. Journal of Social Work Practice, 33(2), 20I-2 I5. doi: I0.1080/02650533.20I9.1608429

Caverlee, J., Liu, L., \& Webb, S. (2008). Socialtrust: tamper-resilient trust establishment in online communities. Proceedings of the 8th ACMIEEECS Joint Conference on Digital Libraries, I04-II 3. 
doi: $10.1 \mid 45 / 1378889.1378908$

Ceaser, D. K. (20I8). 'Because the ego started to grow bigger than the project itself': a case study of founder's syndrome on an educational community of practise. Ethnography and Education, I3(4), 459-476. doi: I0.I080/I7457823.20 I7.I38432 I

Chang, H. H., \& Chuang, S. S. (20I I). Social capital and individual motivations on knowledge sharing: Participant involvement as a moderator. Information and Management, 48(I), 9-18. doi: 10.1016/j.im.2010.11.00I

Chen, C. J., \& Hung, S.W. (2010). To give or to receive? Factors influencing members' knowledge sharing and community promotion in professional virtual communities. Information and Management, 47(4), 226-236. doi: I0.10I6/j.im.20I0.03.00I

Chen, G., Yang, S., \& Tang, S. (20I3). Sense of virtual community and knowledge contribution in a P3 virtual community. Internet Research, 23(I), 4-26. doi: I 0. I I 08/ I 066224 I 3 I I 295755

Chen, I.Y. L., Chen, N.-S., \& Kinshuk. (2009). Examining the factors influencing participants' knowledge sharing behavior in virtual learning communities. Educational Technology and Society, I 2(I), I34-I48.

Chin,W.W. (1998). The Partial Least Squares Approach to Structural Equation Modeling. In Modern Methods for Business Research, (295-336). London: Lawrence Erlbaum Associates Publishers.

Chiu, C., Hsu, M., \& Wang, E. (2006). Understanding knowledge sharing in virtual communities: An integration of social capital and social cognitive theories. Decision Support Systems, 42(3), |872-1888. doi: 10.1016/j.dss.2006.04.00|

Chiu, C. M., Huang, H.Y., Cheng, H. L., \& Sun, P. C. (20I5). Understanding online community citizenship behaviors through social support and social identity. International Journal of Information Management, 35(4), 504-519. doi: I0.1016/j.jijinfomgt.2015.04.009

Cho, H., Gay, G., Davidson, B., \& Ingraffea, A. (2007). Social networks, communication styles, and learning performance in a CSCL community. Computers and Education, 49(2), 309-329. doi: I0.1016/j.compedu.2005.07.003

Christson, A., \& Adedoyin,A. (2016). Deploying virtual communities of practice as a digital tool in social work: a rapid review and critique of the literature. Social Work Education, 35(3), 357-370. doi: 10.1080/026I5479.2016.1 I54660

Clifton, R.A. (1999). The education of university students:A social capital perspective. College Teaching, 47(3), I I4-I I8. Retrieved from http://www.jstor.org/stable/27558954

Cohen,W. M., \& Levinthal, D.A. (1990). Absorptive Capacity:A New Perspective on Learning and Innovation. Administrative Science Quarterly, 35(I), I28. doi: I0.2307/2393553

Conrad, C., \& Poole, M. S. (1998). Strategic organizational communication into the twenty-first century (4th ed.). New York: Harcourt Brace College. 
Cook-Craig, P., \& Sabah,Y. (2009). The role of virtual communities of practice in supporting collaborative learning among social workers. British Journal of Social Work, 39, 725-739. doi: 10.1093/bjsw/bcp048

Correa, J.M., Martínez-Arbelaiz,A. \& Aberasturi-Apraiz, E. (20I5). Post-modern reality shock: Beginning teachers as sojourners in communities of practice. Teaching and Teacher Education, 48, 66-74. doi: 10.1016\%2Fj.tate.2015.02.007.

Correia,A., Paulos, A., \& Mesquita,A. (2009).Virtual communities of practice: examining the motivations and constraints in their knowledge creation and knowledge transfer processes'. OLKC Conference, 8(I), I-16. Retrieved from http://www.feweb.vu.nl/olkc2009/ Papers/7BAnaRamalho.pdf

Couper, G., Denny, H. \& Watkins, A. (20I6). Teaching the Sociocultural Norms of an Undergraduate Community of Practice. TESOL Journal, 7(I), 4-39. doi: 10.1002/tesj. 187

Cowan, J. E., \& Menchaca, M. P. (20I4). Investigating value creation in a community of practice with social network analysis in a hybrid online graduate education program. Distance Education, 35(I), 43-74. doi: I0.1080/0I587919.2014.8938।3

Cross, J. N., \& Cummings, J. (2003). Tie and Network Correlates of Individual Performance in Knowledge-Intensive Work. The Academy of Management Journal, 47(6), 928-937. doi: $10.2307 / 20159632$

Crowley, C., Mcadam, M., Cunningham, J.A., \& Hilliard, R. (2018). Community of Practice : A flexible construct for understanding SME networking roles in the Irish artisan cheese sector. Journal of Rural Studies, 64(August), 50-62. doi: 10.1016/j.jrurstud.2018.08.014

Crues, R.L., Crues, S.R. \& Steinert,Y. (2018). Medicine as a Community of Practice: Implications for Medical Education. Academic Medicine, 93(2), I85-191. doi: I0.1097/ ACM.0000000000001826.

Cundill, G., Roux, D.J. \& Parker, J.N. (20I5). Nurturing communities of practice for transdisciplinary research. Ecology and Society, 20(2), 22. http://dx.doi.org/ I0.575 I/ES-07580200222

Curran, J.A., \& Murphy,A. L. (2009). Bridging the Gap : Knowledge Seeking and Sharing in a Virtual Community of Emergency Practice. Evaluation \& the Health Professions, 32(3), 3|4-327. doi: 10.1 I77/0|63278709338570

Dahlander, L., \& Frederiksen, L. (2012). The Core and Cosmopolitans:A Relational View of Innovation in User Communities. Organization Science, 23(4), 988-I007. doi: I0. I 287/ orsc. III 0.0673

Dainton, M., \& Zelley, E. D. (2005). Applying communication theory for professional life:A practical introduction. Thousand Oaks: Sage publications.

Dawson, S. (2008). A study of the relationship between student social networks and sense of community. Educational Technology \& Society, I I (3), 224-238. 
Day, D.V., Gronn, P., \& Salas, E. (2004). Leadership capacity in teams. Leadership Quarterly, I5(6), 857-880. doi: I0.1016/j.leaqua.2004.09.001

Dennen, V. (20I4). Becoming a blogger:Trajectories, norms, and activities in a community of practice. Computers in Human Behavior, 36, 350-358. doi: I0.1016/j.chb.20 I4.03.028

Deursen,A. J.A. M.Van, Dijk, J.A. G. M.Van, \& Peters, O. (20I I). Rethinking Internet skills:The contribution of gender, age, education, Internet experience, and hours online to mediumand content-related Internet skills. Poetics, 39(2), I25-144. doi: I0.10 I6/j.poetic.20 I I.02.00 I

Dholakia, U. M., Bagozzi, R. P., \& Pearo, L. K. (2004). A social influence model of consumer participation in network- and small-group-based virtual communities. International Journal of Research in Marketing, 2 I (3), 24I-263. doi: I0.I0I6/j.ijresmar.2003.I2.004

Dolinska A. \& D'Aquino, P. (20I6). Farmers as agents in innovation systems. Empowering farmers for innovation through communities of practice. Agricultural Systems, I42, I22-I 30. http://dx.doi.org/10.1016/j.agsy.2015.1 I.009

Doran, D. (20I5). On the discovery of social roles in large scale social systems. Social Network Analysis and Mining, 5(I), I-I8. doi: 10.1007/s I3278-0I5-0290-0

Drew, J., McCallum, B., \& Roggenhofer, S. (2016). Journey to lean: making operational change stick. Springer.

Drohomeretski, E., Gouvea Da Costa, S. E., Pinheiro De Lima, E., \& Garbuio, P.A. (20I4). Lean, six sigma and lean six sigma:An analysis based on operations strategy. International journal of Production Research, 52(3), 804-824. doi: 10.1080/00207543.2013.8420I5

du Plessis, M. (2008). The strategic drivers and objectives of communities of practice as vehicles for knowledge management in small and medium enterprises. International Journal of Information Management, 28(I), 6I-67. doi: I0.10 I6/j.ijinfomgt.2007.05.002

Du Plessis, M. (2005). Drivers of knowledge management in the corporate environment. International Journal of Information Management, 25(3), 193-202. doi: 10.1016/j. ijinfomgt.2004.12.00I

Dubé, L., Bourhis, A., \& Jacob, R. (2005). The impact of structuring characteristics on the launching of virtual communities of practice. Journal of Organizational Change Management, I8(2), |45-I66. doi: I0.1 108/09534810510589570

Duncan-Howell, J. (2007). Online Communities of Practice and their Role in the Professional Development of Teachers (Doctoral dissertation). Retrieved from https://core.ac.uk/ download/pdf/I08852II.pdf

Dwyer, C., Hiltz, S. R., \& Passerini, K. (2007). Trust and privacy concern within social networking sites:A comparison of Facebook and MySpace. Proceedings of the Thirteenth Americas Conference on Information Systems (AMCIS), (January), Paper 339.

Edelmann, N., Krimmer, R., \& Parycek, P. (20I7). How Online Lurking Contributes Value to A 
Conceptual Approach to Evaluating the Role of Lurkers in E-Participation. In 2017 Fourth International Conference on eDemocracy \& eGovernment (ICEDEG) (pp. 86-93). IEEE. doi: I0. I I09/ICEDEG.2017.79625 I 7

Ellemers, N., Gilder, D. De, Haslam, S.A., \& Gilder, D. D. E. (20I4). Motivating Individuals and Groups at Work:A Social Identity Perspective on Leadership and Group Performance.The Academy of Management Review, 29(3), 459-478. doi: http://dx.doi.org/ 10.2307/20 I59054

Elmer, G., Langlois, G., \& Redden, J. (20I5). Compromised data: From social media to big data. (Eds.). Bloomsbury Publishing USA.

Emerson, R. M. (1976). Social Exchange Theory. Annual Review of Sociology, 2, 335-362. doi: 10.3982/ECTA7I95

Erhan, L., Ndubuaku, M., Ferrara, E., Richardson, M., Sheffield, D., Ferguson, F. J., ... Liotta, A. (2019). Analyzing Objective and Subjective Data in Social Sciences: Implications for Smart Cities. IEEE Access, 7, 19890-19906. doi: I0.I I09/ACCESS.2019.2897217

European Commission. (2016). A New Skills Agenda for Europe:Working together to strengthen human capital, employability and competitiveness. Retrieved from https://eur-lex.europa.eu/ legal-content/EN/TXT/?uri=CELEX:520 I6DC038 I

Evans, O., \& Steptoe,A. (2002). The contribution of gender-role orientation, work factors and home stressors to psychological well-being and sickness absence in male- and femaledominated occupational groups. Social Science and Medicine, 54(4), 48I-492. doi: 10.1016/ S0277-9536(0I)00044-2

Evans, C., Yeung, R., Markoulakis, R. \& Guilcher, S. (20I4).An Online Community of Practice to Support Evidence-Based Physiotherapy Practice in Manual Therapy. Journal of Continuing Education in the Health Professions, 34(4), 215-223. I doi: I0.1002/chp.21 253

Fadel, K. J., \& Durcikova,A. (20I4). Enhancing the Motivation, Opportunity, and Ability of Knowledge Workers to Participate in Knowledge Exchange. Proceedings of 47th Hawaii International Conference on System Sciences, 3605-36 I4. doi: I0.I I09/HICSS.20I4.449

Fagnan, J., Rabbany, R., Takaffoli, M.,Verbeek, E., \& Zaïane, O. R. (20 I4). Community Dynamics: Event and Role Analysis in Social Network Analysis.Advanced Data Mining and Applications. Proceedings of Advanced Data Mining and Applications - I Oth International Conference. doi: 10.1007/978-3-319-147|7-8_7

Faraj, S., von Krogh, G., Monteiro, E., \& Lakhani, K. R. (20I6). Online Community as Space for Knowledge Flows. Information Systems Research, 27(4), 668-684. doi: I0.I 287/isre.2016.0682

Feenberg,A., \& Bakardjieva, M. (2004).Virtual community: No “killer implication.” New Media and Society, 6(I), 37-43. doi: I0.I I77/I46/444804039904

Figueiredo, B., \& Scaraboto, D. (20I6). The systemic creation of value through circulation in collaborative consumer networks. Journal of Consumer Research, 43, I-76. doi: I0.1093/jcr/ ucw038 
Fishbein, M., \& Ajzen, I. (1975). Belief, attitude, intention and behavior:An introduction to theory and research. (Addison-Wesley., Ed.). Boston.

Fisher,W.A., Fisher, J. D., \& Harman, J. (2003). The Information - Motivation - Behavioral Skills Model :A General Social Psychological Approach to Understanding and Promoting Health Behavior. In Social Psychological Foundations of Health and Illness (pp. 82-106). doi: 10.1002/9780470753552.ch4

Fiske, D.W. (1982). Convergent-discriminant validation in measurements and research strategies. In D. B. \& L. H. Kidder (Ed.), Forms of validity in research (pp. 77-92). San Francisco: Jossey-Bass.

Flaman, L. M., Nykiforuk, C. I. J., Plotnikoff, R. C., \& Raine, K. (2010). Exploring facilitators and barriers to individual and organizational level capacity building: Outcomes of participation in a community priority setting workshop. Global Health Promotion, I 7(2), 34-43. doi: I0.1177/1757975910365225

Fong, P. S.W., \& Kwok, C.W. C. (2009). Organizational Culture and Knowledge Management Success at Project and Organizational Levels in Contracting Firms. Journal of Construction Engineering and Management, 135(December), I348-1356. doi: 10.106 I/(ASCE)CO.19437862.0000106

Fornell, C. G., \& Larcker, D. F. (I98I). Evaluating structural equation models with unobservable variables and measurement error. Journal of Marketing Research, I8(I), 39-50. doi: $10.2307 / 3151312$

Foss, N.J., Minbaeva, D.B. Pedersen, T., Reinholt, M. (2009). The later, the more deceptive? A patagon dated 1628 from Chateau-Regnault with a Brabantian obverse. Human Resource Management, 48(6), 87I-893. doi: I0.1002/hrm

Freeman, L. C. (1978). Centrality in Social Networks Conceptual Clarification. Social Networks, I(I968), 2I 5-239. doi: I0.10I6/0378-8733(78)9002I-7

Frohlich, M.T. (2002). Techniques for improving response rates in OM survey research. Journal of Operations Management, 20(I), 53-62. doi: I0.1016/S0272-6963(02)00003-7

Fulk, J., Schmitz, J., and Steinfield, C.A. (1990). A Social Influence Model ofTechnology Use. Organizations and Communication Technology. Newbury Park, CT: Sage Publications.

Fuller, J. B., Marler, L. E., \& Hester, K. (2006). Promoting felt responsibility for constructive change and proactive behavior: Exploring aspects of an elaborated model of work design. Journal of Organizational Behavior, 27(8), I089-I I20. doi: 10.1002/job.425.

Fung, M., Boushey, R.P., Watters, J., Morash, R., Smylie, J., Morash, C., DeGrasse, C., Sundaresan, S. (20I4). Piloting a regional collaborative in cancer surgery using a "community of practice" model. Current Oncology, 2 I (I), 27-34. https://dx.doi.org// 0.3747\%2Fco.2 I. I663

Gagnè, M. (2003). The Role of Autonomy Support and Autonomy Orientation in Prosocial Behavior Engagement. Motivation and Emotion, 27(3), 199-224. 
Gannon-Leary, P. \& Fontainha, E. (2007). Communities of Practice and virtual learning communities: benefits, barriers and success factors. Barriers and Success Factors. eLearning Papers, 5, 20-29. Retrieved from: https://papers.ssrn.com/sol3/Delivery.cfm/SSRN_ ID I0 $18066 \_c o d e 4 \mid$ 0864.pdf?abstractid $=|0| 8066 \&$ mirid $=$ |

Garas, A., Garcia, D., Skowron, M., \& Schweitzer, F. (20I2). Emotional persistence in online chatting communities. Scientific Reports, 2(402), I-34. doi: I0.1038/srep00402

Garrison, D. R., \& Arbaugh, J. B. (2007). Researching the community of inquiry framework: Review, issues, and future directions. Internet and Higher Education, 10(3), I57-I 72. doi: 10.1016/j.iheduc.2007.04.001

Garson, G. D. (2016). Partial Least Squares: Regression \& Structural Equation Models. Statistical Publishing Associates.

Gee, J. P., Hull, G., \& Lankshear, C. (2018). The new work order: Behind the language of the new capitalism. In The New Work Order (pp. I-I8I). Taylor and Francis.

Gholami, B. (20I5). Self-Organizing, Social and Adaptive Nature of Agile Information Systems Development Teams: Essays on Leadership and Learning (Doctoral dissertation). Retrieved from https://madoc.bib.uni-mannheim.de/3969I/I/DissertationBG.pdf

Gilbuena, D.M., Sherrett, B.U., Gummer, E.S., Champagne,A.B. \& Koretski, M.D. (20I5). Feedback on Professional Skills as Enculturation into Communities of Practice. Journal of Engineering Education, 104(I), 7-34. doi: I0.1002/jee.2006I.

Gilchrist, a. (2000). The well-connected community: networking to the edge of chaos. Community Development Journal, 35(3), 264-275. doi: I0.1093/cdj/35.3.264

Gleave, E.,Welser, H.T., Lento, T. M., \& Smith, M.A. (2009). A Conceptual and Operational Definition of "Social Role" in Online Community. In 42nd Hawaii International Conference on System Sciences (PP. I-I I). doi: I0.I I09/HICSS.2009.6

Godin, G., \& Kok, G. (1996). The theory of planned behavior: a review of its applications to health-related behaviors. American Journal of Health Promotion, I I (2), 87-98. doi: |0.4278/0890-I| |7|-|| I.2.87

Goffman, E. (1959). The presentation of self in everyday life. New York: Doubleday Anchor Books.

Goldie, J. G. S. (2016). Connectivism:A knowledge learning theory for the digital age? Medical Teacher, 38(I0), I064-1069. doi: I0.3109/0I42I59X.2016.II7366I

Gonçalves, D., Nogueira, I.C. \& da Silva, M.C.V. (2016). Inducing Supervision Practices among peers in a Community of Practice. Journal for Educators Teachers and Trainers, 7(2), 108-II9. Retrieved from: http://jett.labosfor.com/index.php/jett/article/download/234/29 I

Gongla, P., \& Rizzuto, C. R. (200I). Evolving communities of practice: IBM Global Services experience. IBM Systems Journal, 40(4), 842-862. doi: I0.I I47/sj.404.0842 
González-Howard, M. \& McNeill, K.L. Learning in a community of practice: Factors impacting english-learning students' engagement in scientific argumentation. Journal of Research in Science Teaching, 53(4), 527-553. doi: 10.1002/tea.21 310.

Goodyear,V.A., Casey,A. \& Kirk, D. (20I4). Tweet me, message me, like me: using social media to facilitate pedagogical change within an emerging community of practice. Sport, Education and Society, I9(7), 927-943. http://dx.doi.org/I0.1080/I3573322.20I3.858624

Goodyear,V.A. \& Casey,A. (20I5). Innovation with change: developing a community of practice to help teachers move beyond the 'honeymoon' of pedagogical renovation. Physical Education and Sport Pedagogy, 20(2), 186-203. doi: I0.1080/I7408989.20 I3.8I 7012

Granovetter, M. (1973). The Strength ofWeak Ties. The American Journal of Sociology. doi: $10.1086 / 225469$

Grant, R. M. (1996). Toward a Knowlegdge-Based Theory of the Firm. Strategic Management Journal, I7(S2), 109-122. doi: 10.1002/smj.4250171 I I0

Gruen,T.W., \& Czaplewski,A.J. (2005). How e-communities extend the concept of exchange in marketing: An application of the motivation, opportunity, ability ( MOA ) theory, Marketing Theory, 5(I), 33-49. doi: I0.I I77/I470593 I05049600

Gruen,T.W., Osmonbekov, T., \& Czaplewski,A. J. (2007). Customer-to-customer exchange: Its MOA antecedents and its impact on value creation and loyalty. Journal of the Academy of Marketing Science, 35(4), 537-549. doi: I0. I007/s I I747-006-00 I2-2

Guerin, C., Carter, S. \& Aitchison, C. (2015). Blogging as community of practice: lessons for academic development? International Journal for Academic Development, 20(3), 21 2-223. http:// dx.doi.org/I0.1080/I360I44X.20I5.1042480

Guptill, J. (2005). Knowledge management in health care. Journal of Health Care Finance, 3I(3), I0-I5. Retrieved from http://www.kmatwork.com/images/Journal_HC_Finance_ article_4-05.pdf

Haas, M. R., \& Hansen, M.T. (2007). Different knowledge, different benefits:Toward a productivity perspective on knowledge sharing in organizations. Strategic Management Journal, 28(I I), II33-II53. doi: I0.1002/smj.63।

Hafeez, K., \& Alghatas, F. (2007). Knowledge Management in a Virtual Community of Practice using Discourse Analysis. The Electronic Journal of Knowledge Management, 5(I), 29-42.

Retrieved from http://www.ejkm.com/issue/download.html?idArticle=86

Hair, J. F., Ringle, C. M., \& Sarstedt, M. (20I I). PLS-SEM: Indeed a Silver Bullet. The Journal of Marketing Theory and Practice, I9(2), I39-I52. doi: I0.2753/MTPI069-6679I 90202

Ham, M., Jeger, M., \& Ivković, A. F. (20I5). The role of subjective norms in forming the intention to purchase green food. Economic Research-Ekonomska Istraživanja, 28(I), 738-748. doi: I0.1080/I33I677X.20I5.1083875 
Han, J. J., Zheng, R. J., \& Xu,Y. (2007). The effect of individual needs, trust and identification in explaining participation intentions in virtual communities. In Proceedings of the Annual Hawaii International Conference on System Sciences (Pp. I-10). doi: I 0.I I09/HICSS.2007.525

Han, J., Kamber, M., \& Pei, J. (2012). Data Mining: Concepts and Techniques. San Francisco, CA, itd: Morgan Kaufmann. doi: I0.10I6/B978-0-12-38I479-I.0000I-0

Han, Y., \& Tang, J. (20I5, August). Probabilistic community and role model for social networks. In Proceedings of the 2 I th ACM SIGKDD international conference on Knowledge Discovery and Data Mining (pp. 407-4I6).ACM. doi: I0.I I45/2783258.2783274

Hara, N., \& Hew, K. F. (2007). Knowledge-Sharing in an Online Community of Health-care Professionals. Knowledge-Sharing Among Health-Care Professionals, 20(3), 235-26I. doi: I0.1 I08/095938407I0822859

Harris, B. J. K. (20I8). Building a Useful Exponential Random Graph Model. Sage Publications.

Harris, G. (20I5). Participation in Online Communities: Reconfiguring Relations of Participation. Retrieved from http://unsworks.unsw.edu.au/fapi/datastream/unsworks:3499I/ SOURCE02?view=true

Haslam, S.A., Postmes, T., \& Ellemers, N. (2003). More than a Metaphor: Organizational Identity Makes Organizational Life Possible. British Journal of Management, I4, 357-369. doi: I0.1 I I I/j.| 467-855I.2003.00384.x

Heinz, M., \& Rice, R. E. (2009). An Integrated Model of Knowledge Sharing in Contemporary Communication Environments. Annals of the International Communication Association, 33(I), |34-I75. doi: |0.1080/23808985.2009.| | 679086

Henseler, Jörg; Ringle, Christian M.; \& Sinkovics, R. R. (2009). The use of partial least squares path modeling in international marketing. New Challenges to International Marketing. Advances in International Marketing, 20, 277-3 I9. doi: I0. I I08/SI474-7979(2009)00000200 I4

Henseler, J., \& Sarstedt, M. (2013). Goodness-of-fit indices for partial least squares path modeling. Computational Statistics, 28(2), 565-580. doi: I0.1007/s00 I80-0 I2-03 I 7-I

Hew, K. F., \& Hara, N. (2007). Empirical study of motivators and barriers of teacher online knowledge sharing. Educational Technology Research and Development, 55(6), 573-595. doi: |0.1007/s | |423-007-9049-2

Hildreth, P., \& Kimble, C. (2004). Knowledge Networks: Innovation Through Communities of Practice. Heshey: USA: Idea Group Publishing.

Hindman, M. (2009). The Myth of Digital Democracy. New Jersey: Princeton University Press. Retrieved from http://press.princeton.edu/titles/878I.html

Hirschi, T. (2002). Causes of Delinquency. New Brunswick. NJ:Transaction Publishers.

Hlapanis, G., \& Dimitracopoulou, A. (2007). The school-teacher's learning community: 
matters of communication analysis. Technology Pedagogy and Education, I6(2), I33-I52. doi: I0.1108/0262I710410558468

Hoadley, C. (2012). What is a Community of Practice and How Can We Support It? Theoretical Foundations of Learning, 287-300. doi: I0.1080/09523987.2012.703429

Hoffmann, T., Desha, L., \& Verrall, K. (20I I). Evaluating an online occupational therapy community of practice and its role in supporting occupational therapy practice. Australian Occupational Therapy Journal, 58(5), 337-345. doi: I0. I I I I/j. I440-I630.20 I I.00954.x

Holt Larsen, H. (2004). Global career as dual dependency between the organization and the individual. Journal of Management Development, 23(9), 860-869. doi: I0.1080/I475939070|406745

Holton, E. H., \& Burnett, M. B. (1997). Qualitative research methods. Human resource development research handbook: Linking research and practice. San Francisco: BerrettKoehler Publishers.

Homans, G. C. . (1958). Social Behavior as Exchange. American Journal of Sociology, 63(6), 597-606.

Hooff, Bart Van Den, \& Ridder, J. a. De. (2004). Knowledge sharing in context: the influence of organizational commitment, communication climate and CMC use on knowledge sharing. Journal of Knowledge Management, 8(6), I I7-I30. doi: I0.I I08/I36732704I0567675

Hou, H. (20I5). What makes an online community of practice work? A situated study of Chinese student teachers' perceptions of online professional learning. Teaching and Teacher Education, 46, 6- I6. http://dx.doi.org/10.1016/j.tate.2014.10.005

Hrastinski, S. (2009). A theory of online learning as online participation. Computers and Education, 52(I), 78-82. doi: 10.1016/j.compedu.2008.06.009

Hsiao, C. C., \& Chiou, J.S. (20I2). The impact of online community position on online game continuance intention: Do game knowledge and community size matter? Information and Management, 49(6), 292-300. doi: 10.1016/j.im.2012.09.002

Hughes, J. H. J. (2007). The Ability-Motivation-Opportunity Framework for Behavior Research in IS. In proceedings of the 40th Annual Hawaii International Conference on System Sciences (HICSS'07), I-I0. doi: I0.I I09/HICSS.2007.518

Hulland, J. (1999). Use of Partial Least Squarres (PLS) in Strategic Management Research:A Review of Four Recent Studies. Strategic Management Journal, 20, 195-204. doi: I0.1002/ (SICI) I097-0266(I 99902)20:2< 195::AID-SMJ I 3>3.0.CO;2-7

Hung, K., \& Petrick, J. F. (20I I). Investigating the role of motivation , opportunity and ability ( MOA ) on travel intentions :An application of the MOA model in cruise tourism. In proceedings of the ttra International Conference (Pp. I-I3). doi: I0.2307/23934 I4

Hung, K., Sirakaya-Turk, E., \& Ingram, L. J. (20I I). Testing the Efficacy of an Integrative 
Model for Community Participation. Journal of Travel Research, 50(3), 276-288. doi: I0. I |77/00472875 I036278|

Hung, S., Durcikova, A., Lai, H., \& Lin,W. (20I I). The influence of intrinsic and extrinsic motivation on individuals ' knowledge sharing behavior. Journal of Human Computer Studies, 69(6), 4I5-427. doi: I0.1016/j.ijhcs.20II.02.004

Hung, S., Lai, H., \& Chou,Y. (20I5). Knowledge-sharing Intention in Professional Virtual Communities:A Comparison Between Posters and Lurkers. Journal of the Association for Information Science and Technology, 66(I2), 2494-25 I0. doi: I0.1002/asi.23339

Hurtubise, K., Rivard, L., Berbari, J., Héguy, L., \& Camden, C. (2017). Exploring engagement in a virtual community of practice in pediatric rehabilitation: who are non-users, lurkers, and posters? Disability and Rehabilitation, 4 I (8), I-8. doi: I0. I080/09638288.20 I 7.I4I6496

Huysman, M., \& Wulf,V. (2006). IT to Support Knowledge Sharing in Communities, Towards a Social Capital Analysis. Journal on Information Technology, 2 I ( I), I-5 I. doi: I0. I057/palgrave. jit.2000053

Ibarra, H., \& Andrews, S. B. (1993). Power, Social Influence, and Sense Making: Effects of Network Centrality and Proximity on Employee Perceptions. Administrative Science Quarterly, 38(2), 277-303. doi: 10.2307/23934I4

Ifinedo, P. (20I4). Information \& Management Information systems security policy compliance : An empirical study of the effects of socialisation, influence, and cognition. Information \& Management, 5 I (I), 69-79. doi: I0.1016/j.im.2013.10.00I

Iriberri, A., \& Leroy, G. (2009). A life-cycle perspective on online community success. ACM Computing Surveys, 4 I (2), I-29. doi: I0.I I45/I459352.I459356

Jahnke, I. (2010). Dynamics of social roles in a knowledge management community. Computers in Human Behavior, 26(4), 533-546. doi: 10.1016/j.chb.2009.08.010

Jeon, S. H., Kim, Y. G., \& Koh, J. (20I I). Individual, social, and organizational contexts for active knowledge sharing in communities of practice. Expert Systems with Applications, 38(10), |2423-|243I. doi: I0.1016/j.eswa.20II.04.023

Jeppesen, L. B., \& Lakhani, K. R. (20I0). Marginality and problem-solving effectiveness in broadcast search. Organization science, 2 I (5), I0 I6- I033. doi: I0. I287/orsc. 1090.049 |

Jian, G., \& Jaffres, L.W. (2006). Understanding employees' willingness to contribute to shared electronic databases:A three-dimensional framework. Communication Research, 33(4), 242-26I. doi: |0.| |77/0093650206289|49

Jiménez-Zarco,A. I., González-González, I., Saigí-Rubió, F., \& Torrent-Sellens, J. (20I4).The co-learning process in healthcare professionals:Assessing user satisfaction in virtual communities of practice. Computers in Human Behavior, 5 I, I303-13 I3. doi: 10.10 I6/j. chb.20I4.II.057 
Jin, Q. (2002). Design of a virtual community based interactive learning environment. Information Sciences, I40(I-2), I7I-19I. doi: I0.10I6/S0020-0255(0I)00I86-4

Johnson, S. L., Safadi, H., \& Faraj, S. (2015). The emergence of online community Leadership. Information and Organization, (July), 35-68. doi: I0.1287/isre.20I4.0562

Johnston, J. (2017). Courts' use of social media:A community of practice model. International Journal of Communication, I I , 669-683. Retrieded from https://ijoc.org/index.php/ijoc/article/ view/6I2I.

Kalafatis, S.E., Lemos, M.C., Lo, Y.J. \& Frank, K.A. (20I5). Increasing information usability for climate adaptation:The role of knowledge networks and communities of practice. Global Environmental Change, 32, 30-39. http://dx.doi.org/I0.1016/j.gloenvcha.2015.02.007.

Kane, G. C., Palmer, D., Philips Nguyen, A., Kiron, D., \& Buckley, N. (20I5). Strategy, Not Technology, Drives Digital Transformation. MIT Sloan Management Review \& Deloitte, (57I8I), 27. doi: http:/www2.deloitte.com/content/dam/Deloitte/cn/Documents/technology-mediatelecommunications/deloitte-cn-tmt-strategy-not-technology-drive-digital-transformationen-I50930.pdf

Kang,Y. J., Lee, J.Y., \& Kim, H.W. (20I7). A psychological empowerment approach to online knowledge sharing. Computers in Human Behavior, 74, 175-187. doi: 10.1016/j. chb.2017.04.039

Kankanhalli,A., Tan, B., \& Wei, K. (2005). Contributing Knowledge to Electronic Knowledge Repositories:An Empirical Investigation. Information Technologies and Knowledge Management, 29(I), I |3-|43. doi: I0.2307/25|48670

Kaplan, A. M., \& Haenlein, M. (20I0). Users of the world, unite! The challenges and opportunities of Social Media. Business Horizons, 53(1), 59-68. doi: 10.1016/j. bushor.2009.09.003

Karam, R., Straus, S. G., Byers, A., Kase, C.A., \& Cefalu, M. (20I8). The role of online communities of practice in promoting sociotechnical capital among science teachers. Educational Technology Research and Development, 66(2), 215-245. doi: 10.1007/s I I 423-0 I7954I-2

Katz, E., Blumler, J. G., \& Gurevitch, M. (1973). Uses and Gratifications Research. Public Opinion Quarterly, 37(4), 509-523. doi: 10.1086/268109

Kensington-Miller, B. (20I8). Surviving the first year: new academics flourishing in a multidisciplinary community of practice with peer mentoring. Professional Development in Education, 44(5), 678-689. http://doi.org/I0.1080/I94I5257.20 I7.I387867

Kettinger,W. J., Li,Y., Davis, J. M., \& Kettinger, L. (20I5). The roles of psychological climate, information management capabilities, and IT support on knowledge-sharing: an MOA perspective. European Journal of Information Systems, 24(I), 59-75. doi: I0. I057/ejis.2013.25

Kim, S. J., Hong, J.Y., \& Suh, E. H. (20I2).A diagnosis framework for identifying the current 
knowledge sharing activity status in a community of practice. Expert Systems with Applications, 39(18), I 3093-13107. doi: 10.1016/j.eswa.2012.05.092

Kimmerle, J., Thiel, A., Gerbing, K. K., Bientzle, M., Halatchliyski, I., \& Cress, U. (20I3).

Knowledge construction in an outsider community: Extending the communities of practice concept. Computers in Human Behavior, 29(3), I078-1090. doi: I0. I0I6/j.chb.20I2.09.0I0

Koh, J., Kim,Y., Butler, B., \& Bock, G. (2000). Encouraging participation. Ca-A Cancer Journal for Clinicians, 50(2), 69-74. doi: I0.1016/j.jclinepi.2007.09.00 I

Koh, J., \& Kim,Y. G. (2004). Knowledge sharing in virtual communities:An e-business perspective. Expert Systems with Applications, 26(2), I55-166. doi: I0.10 I6/S09574I74(03)00II6-7

Kovanovic, V., Joksimovic, S., Gašević, D., \& Hatala, M. (20I4).What is the Source of Social Capital ? The Association Between Social Network Position and Social Presence in Communities of Inquiry. In Proceedings of the Workshop on Graph-Based Educational Data Mining at Educational Data Mining Conference (G-EDM 2014), I I83, I-8. Retrieved from http://www.scopus.com/inward/record.url?scp=849223 I8465\&partnerID=8YFLogxK

Kratzer, J., Lettl, C., Franke, N., \& Gloor, P.A. (2016). The Social Network Position of Lead Users. Journal of Product Innovation Management, 33(2), 20 I-2 I6. doi: I0. I I I //jpim. I 229 I

Kraut, R. E., \& Resnick, P. (20I I). Building Successful Online Communities. London, England: MIT Press.

Kruse, S., \& Louis, K. (1993). An emerging framework for analyzing school-based professional community. Annual Meeting of the American Educational Research Association, (April), 23-44. Retrieved from https://files.eric.ed.gov/fulltext/ED358537.pdf

Kulkarni, U., Ravindran, S., \& Freeze, R. (2007).A knowledge management success model: theoretical development and empirical validation. Journal of Management Information Systems, 23(3), 309-347. doi: I0.2753/MIS0742-I2222303 |

Kwong-Kay Wong, K. (20I3). Partial Least Squares Structural Equation Modeling (PLSSEM) Techniques Using SmartPLS. Marketing Bulletin, 24(I), I-32. doi: I0. I097/ MNM.0b0I 3 e328332f80I

Lampe, C.,Wash, R., Velasquez,A., \& Ozkaya, E. (20l0). Motivations to participate in online communities. In Proceedings of the SIGCHI Conference on Human Factors in Computing Systems, 1927-1936. doi: I0.1 I45/I753326.17536I6

Lampel, J. \& Bhalla,A. (2007). The Role of Status Seeking in Online Communities : Giving the Gift of Experience. Journal of Computer-Mediated Communication, I2, 434-455. doi: I0.1 I I I/j.1083-610I.2007.00332.x

Lancini, A. (20I5). Evaluating Interorganizational Knowledge Management:The concept of IKM orientation. Electronic Journal of Knowledge Management, I3(2), I I7-I29. 
Lane, N. D., Pengyu, L., Zhou, L., \& Zhao, F. (20I4). Connecting personal-scale sensing and networked community behavior to infer human activities. In Proceedings of the 20/4 ACM International Joint Conference on Pervasive and Ubiquitous Computing - UbiComp '1 4 Adjunct, 595-606. doi: I0.1 |45/2632048.2636094

Langer, R., \& Beckman, S. C. (2005). Sensitive research topics: Netnography revisited. Qualitative Market Research, 8(2), I89-203. doi: I0.I I08/I35227505। 0592454

Lau, K.W., Lee, P.Y., \& Chung,Y.Y. (2019). Leadership \& Organization Development Journal A collective organizational learning model for organizational development Article information Article information: Leadership \& Organization Development Journal, 40(I), I07-123. doi: https:// doi.org/I0.I I08/LODJ-06-2018-0228

Lave, J., \&Wenger, E. (199I). Situated learning: Legitimate peripheral participation. Cambridge university press.

Lee, C., \& Ma, L. (20I2). News sharing in social media:The effect of gratifications and prior experience. Computers in Human Behavior, 28(2), 33I-339. doi: I0.10 I6/j.chb.20I I.10.002

Lee, L. L., \& Neff, M. (2004). How Information Technologies Can Help Build and Sustain an Organization's CoP: Spanning the Socio-Technical Divide? Knowledge Networks: Innovation Through, I65-183. doi: I0.4018/978-I-59|40-200-8.ch0I5

Lee, L., Reinicke, B., Sarkar, R., \& Anderson, R. (20I5). Learning Through Interactions: Improving Project Management Through Communities of Practice. Project Management Journal, 7(3), 40-52. doi: 10.1002/pmj

Leonardi, P. M. (20I4). Social media, knowledge sharing, and innovation:Toward a theory of communication visibility. Information Systems Research, 25(4), 796-8I6. doi: I0. I287/ isre.2014.0536

Lesser E. L. and Storck, J. (200I). Community of practice and organizational Performance. IBM Systems Journal, 40, 83 I-84I. doi: I0.I I47/sj.404.083 I

Levina, N., \& Arriaga, M. (20I4). Distinction and Status Production on User-Generated Content Platforms: Using Bourdieu's Theory of Cultural Production to Understand Social Dynamics in Online Fields. Information Systems Research, 25(3), 468-488. doi: I0. I 287/isre.20 I 4.0535

Lewis, W., Agarwal, R., \& Sambamurthy, V. (2003). Sources of influence on beliefs about information technology use:An empirical study of knowledge workers. MIS Quarterly, 27(4), 657-678. doi: 10.2307/30036552

Lewis, R. \& Kelly, S. (20I8). GP/GPN partner* perspectives on clinical placements for student nurses in general practice: Can a community of practice help to change the prevailing culture within general practice? BMC Family Practice, I9(I), I-I I. doi: I0. I I86/s I 2875-0 I80842-2.

Li, L. C., Grimshaw, J. M., Nielsen, C., Judd, M., Coyte, P. C., \& Graham, I. D. (2009). Use of communities of practice in business and health care sectors: a systematic review. 
Liao, S., \& Chou, E. (20I I). Intention to adopt knowledge through virtual communities: posters vs lurkers. Online Information Review, 36(3), 442-46 I. doi: I0. I I08/ I 468452 I 2 I I 24 I440

Liebeskind, J. P. (1996). Knowledge, Strategy, and the Theory of the Firm. Strategic Management Journal, I7, 93-107. doi: 10.1002/smj.4250171 109

Littlejohn, S.W., Foss, K.A., \& Oetzel, J. G. (20I7). Theories of Human Communication. Belmont, CA:Wadsworth.

Liu, L.,Wagner, C., \& Chen, H. (20I4). Determinants of Commitment in an Online Community: Assessing the Antecedents of Weak Ties and Their Impact. Journal of Organizational Computing and Electronic Commerce, 24(4), 27I-296. doi: I0. I080/I09 I9392.20 I4.956609

Lohmöller, J. (1989). Latent variable path modeling with partial least squares. Heidelberg: Physica.

Loyarte, E., \& Rivera, O. (2007). Communities of practice: a model for their cultivation. Journal of Knowledge Management, I I(3), 67-77. doi: I0.I I08/I36732707I0752 I I 7

Lu,Y., Zhao, L., \& Wang, B. (20I0). From virtual community members to C2C e-commerce buyers:Trust in virtual communities and its effect on consumers' purchase intention. Electronic Commerce Research and Applications, 9(4), 346-360. doi: 10.1016/j. elerap.2009.07.003

Lundkvist, A. (2004). User Networks as Sources of Innovation. In Knowledge Networks: Innovation Through (pp. 96-105). doi: 10.4018/978-1-59|40-200-8.ch02 I

Lusher, D., Koskinen, J., \& Robins, G. (20I3). Exponential Random Graph Models for Social Networks. Cambridge university press.

Macia, M., \& García, I. (20I6). Informal online communities and networks as a source of teacher professional development: A review. Teaching and Teacher Education Journal, 55, 29I-307. doi: http://dx.doi.org/I0.1016/j.tate.2016.01.02I

Maclnnis, D. J., \& Jaworski, B. J. ( 1 989). Information Processing from Advertisements: Toward an Integrative Framework. Journal of Marketing, 53(4), I-23. doi: http://dx.doi. org//0.2307//25/376

Maclnnis, D. J., Moorman, C., \& Jaworski, B. J. (1991). Enhancing and measuring consumers' motivation, opportunity, and ability to process brand information from ads. Journal of marketing, 55(4), 32-53. doi: 10.2307//251955

Mahrt, M., \& Scharkow, M. (20I3). The Value of Big Data in Digital Media Research. Journal of Broadcasting and Electronic Media, 57(I), 20-33. doi: I0.1080/08838I5I.20I2.76I700

Malhotra, A., Gosain, S., \& Hars, A. ( 1997). Evolution of a Virtual Community: Understanding Design Issues Through a Longitudinal Study. In Proceedings of the I8th International Conference on Information Systems, 59-73. doi: I0.I.I.I32.538I 
Malinen, S. (20I5). Understanding user participation in online communities:A systematic literature review of empirical studies. Computers in Human Behavior, 46, 228-238. doi: I0.10I6/j.chb.20I5.0I.004

Malone, D. (2002). Knowledge management. A model for organizational learning. International Journal of Accounting Information Systems, 3(2), I I I-I23. doi: I0. I0 I 6/S I467-0895(02)00039-8

Marchand, F., Mey, K. De, Debruyne, L., Haene, K. D., \& Meul, M. (2010). From individual behaviour to social learning: start of a participatory process towards sustainable agriculture. In Proceedings of the 9th European IFSA Symposium, 670-682. Retrieved from https://www. researchgate.net/publication/30325778I_From_individual_behavior_to_social_learning Start_to_a_participatory_process_towards_sustainable_agriculture

Marshall, S. P. (1997). Creating Sustainable Learning Communities for the Twenty-First Century. The Organization of the Future, I77-188. Retrieved from https:/www.decd.sa.gov.au/sites/g/ files/net69l/f/creating_sustainable_learning_communities_for_the_twenty-first_century.pdf

Martin, X., \& Salomon, R. (2003). Knowledge transfer capacity and its implications for the theory of the mul- tinational corporation. Journal of International Business Studies, 34(4), 356-373. doi: 10.1057/palgrave.jibs.8400037

Martinsons, M. G., Davison, R. M., \& Huang, Q. (20I7). Strategic knowledge management failures in small professional service firms in China. International Journal of Information Management, 37(4), 327-338. doi: 10.1016/j.ijinfomgt.2017.04.003

Matarrita-Cascante, D., \& Brennan, M. a. (20I2). Conceptualizing community development in the twenty-first century. Community Development, 43(3), 293-305. doi: I0.1080/I5575330.20II.593267

Mayer-Schonberger,V. \& Cukier, K. (20 I3). Big Data:A Revolution That Will Transform How We Live, Work and Think. John Murray.

Mayrhofen, A., Goodman, C. \& Holman, C. (20I5). Establishing a community of practice for dementia champions (innovative practice). Dementia, I4(2), 259-266. doi: I0.1177/1471301214542534

Mccully,W., \& Lampe, C. (20I I). Online and Offline Interactions in Online Communities. In Proceedings of the 7th International Symposium on Wikis and Open Collaboration (pp. 39-48). doi: | 0. I |45/2038558.2038566

McDermott, R. (2000). Community Development As a Natural Step. Knowledge Management Review, 3(5), I6-19.

Mcdermott, R., \& Archibald, D. (20I I). Harnessing Your Staff's Informal Networks. Exchange Organizational Behavior Teaching Journal, 3-7.

McKnight, D. H. (2005). Trust in information technology. In The Blackwell Encyclopedia of Management, 7, 329-33I. Retrieved from https://msu.edu/user/mcknig26/TrustinTechnology. doc 
McMillan, D.W., \& Chavis, D. M. (1986). Sense of Community:A Definition \& Theory. Journal of Community Psychology, I 4(4), 3 I5-325. Retrieved from https://pdfs.semanticscholar.org/ e5fb/8ece I08aec367| 4ee4I3876e6 Ib05I0e7c80.pdf

Merton, R. K. (1968). The Matthew Effect in Science. Science, I59(3810), 56-63. doi: I0.1 I26/ science. 159.3810 .56

Millen, D. R., Fontaine, M. a, \& Miller, M. J. (2002). Understanding the Benefits and Costs of Communities of Practice. Communications of the ACM, 45(4), 69-73. doi: I0.I |45/505248.505276

Miller, D., \& Proctor, A. (2016). Enterprise Change Management: How to Prepare Your Organization for Continuous Change. Kogan Page Publishers.

Mohtar, R.H. \& Lawford, R. (2016). Present and future of the water-energy-food nexus and the role of the community of practice. Journal of Environmental Studies and Sciences, 6(I), 192199. doi: $10.1007 / \mathrm{s}|34| 2-016-0378-5$.

Morgan, R. M., \& Hunt, S. D. ( 1994). The Commitment-Trust Theory of Relationship Marketing. Journal of Marketing, 58(3), 20-38. doi: 10.2307/I 252308

Morse, J. M. (20I5). “Data Were Saturated ....” Qualitative Health Research, 25(5), 587-588. doi: I0.II77/I0497323I5576699

Nahapiet, J., \& Ghoshal, S. (1998). Social Capital, Intellectual Capital, and the Organizational Advantage. The Academy of Management Review, 23(2), 242-266. doi: 10.2307/259056

Nardi, B.A.,Whittaker, S., \& Schwarz, H. (2002). NetWORKers and their Activity in Intensional Networks. Computer Supported Cooperative Work, I I ( I-2), 205-242. doi: I0.1023/A:I015241914483

Nash, J. E., \& Calonico, J. M. (1996). The meaning of social interaction:An introduction to social psychology. Rowman \& Littlefield Publishers.

Ndlela, L.T., \& Du Toit, A. S.A. (200I). Establishing a knowledge management programme for competitive advantage in an enterprise. International Journal of Information Management, 2 I (2), I5 I-I65. doi: I0.I0I6/S0268-40I2(0I)00007-X

Nias, J., Southworth, G., \& Yeomans, R. (1989). Staff relationships in the primary school:A study of organizational cultures. Continuum International Publishing Group Ltd.

Nickols, F. (2003). Communities of Practice An Overview. Distance Consulting, 6. Retrieved from http://www.nickols.us/CoPOverview.pdf

Nielsen, C. (2018). Relating Successful Business Models to Intellectual Capital and Knowledge Management Practices. Electronic Journal of Knowledge Management, I 6(I), 48-55. doi: I0.10I6/S0024-630I(99)00II5-6

Nistor, N., Baltes, B., Dascalu, M., Mihhaila, D., Smeaton, G., \& Trausan-Matu, S. (20I4). 
Participation in virtual academic communities of practice under the influence of technology acceptance and community factors. A learning analytics application. Computers in Human Behavior, 34, 339-344. doi: 10.1016/j.chb.2013.10.051

Nistor, N., Daxecker, I., Stanciu, D., \& Diekamp, O. (20I5). Sense of community in academic communities of practice: Predictors and effects. Higher Education, 69(2), 257-273. doi: |0.1007/s |0734-0|4-9773-6

Nonaka, I. (1994). A Dynamic Theory of Organizational Knowledge Creation. Organization Science, 5(I), I4-37. doi: 10.1287/orsc.5.1.14

Nonaka, I., \& Toyama, R. (20I5). The knowledge-creating theory revisited: knowledge creation as a synthesizing process. In The essentials of knowledge management (pp. 95- I I0). Palgrave Macmillan, London. doi: I0.1057/palgrave.kmrp.850000 I

Nonaka, I., Toyama, R., \& Konno, N. (2000). SECI , Ba and Leadership : a Unified Model of Dynamic Knowledge Creation. Long Range Planning, 33, 5-34.

Nykvist, B. (20I4). Does Social Learning Lead to Better Natural Resource Management? A Case Study of the Modern Farming Community of Practice in Sweden. Society and Natural Resources, 27(4), 436-450. doi: 10.1080/08941920.2013.861562.

Orlikowski,W. J. (1993). Case Tools As Organizational-Change - Investigating Incremental and Radical Changes in Systems-Development. MIS Quarterly, I 7(3), 309-340. doi: I0.2307/249774

Oshri, I., Fenema, P., \& Kotlarsky, J. (2008). Knowledge transfer in globally distributed teams: the role of transactive memory. Information Systems Journal, I8(4), 593-6 I 6. doi: I0. I I I / j. I 3652575.2007.00243.x

Pan, Y., Xu, Y. C., Wang, X., Zhang, C., Ling, H., \& Lin, J. (20I5). Integrating social networking support for dyadic knowledge exchange:A study in a virtual community of practice. Information and Management, 52(I),6I-70. doi: I0.10I6/j.im.20I4.I0.00 I

Panzarasa, P., Opsahl,T., \& Carley, K. M. (2009). Patterns and dynamics of users' behavior and interaction: Network analysis of an online community. Journal of the American Society for Information Science and Technology, 60(5), 91 I-932. doi: I0.1002/asi.21015

Pareto,V. (I896). Cours d'économie politique: professé à l'Université de Lausanne (Vol. I). Lausanne: F. Rouge.

Park, C.W., \& Mittal, B. (1985). A Theory of Involvement in Consumer Behavior: problems and Issues. Research in Consumer Behavior. Greenwich, United Kingdom: Jai Press.

Parker, S.K.,Wall, T.D., Jackson, P. R. ( 1997). “'That’ s Not My Job “: Developing Flexible Employee Work Orientations. The Academy of Management Journal, 40(4), 899-929. Retrieved from https://www.jstor.org/stable/256952

Pattison, P., \& Wasserman, S. (1996). Logit models and logistic regressions for social networks: 
Psychometrika, 6I(3), 40I-425. doi: I0.I348/0007I I099I59053

Pavlou, P.A., \& Fygenson, M. (2006). Understanding and Predicting Electronic Commerce Adoption:An Extension of the Theory of Planned Behavior. MIS Quarterly, 30(I), I I5-I43. doi: $10.2307 / 25 \mid 48720$

Pearce, C. L., \& Sims, H. P. (2000). Shared leadership:Toward a multi-level theory of leadership. In Advances in interdisciplinary studies of work teams (pp. I 15-139). Emerald Group Publishing Limited. doi: 10.1016/SI572-0977(00)07008-4

Peñarroja,V., Sánchez, J., Gamero, N., Orengo,V., \& Zornoza, A. M. (20I9). The influence of organisational facilitating conditions and technology acceptance factors on the effectiveness of virtual communities of practice. Behaviour and Information Technology, 38(8), I-13. doi: I0.1080/0|44929X.20I8.I564070

Pettigrew, A. M. ( 1979). On studying organizational cultures. Administrative Science Quarterly, 24(4), 570-58I. doi: 10.2307/2392363

Phang, C.W., Kankanhalli,A., \& Sabherwal, R. (2009). Usability and Sociability in Online Communities:A Comparative Study of Knowledge Seeking and Contribution. Journal of the Association for Information Systems, IO(10), 72I-747. Retrieved from https://aisel.aisnet.org/ jais/voll 0/iss 10/2

Pilny, A., \& Proulx, J. (2018). The Influence of Prototypical Communication in Dark Online Organizations: How to Speak Like a Monger. Journal of Language and Social Psychology, 37(2), 249-26I. doi: I0.II77/0261927X I772258|

Plaskoff, J. (2003). Intersubjectivity and community-building: Learning to learn organizationally. In W. Easterby-Smith, M.A. Lyles, \& K. E. (Ed.), The Blackwell handbook of organi- zational learning and knowledge management. Malden, MA: Blackwell Publishers.

Podsakoff, P. M., MacKenzie, S. B., Lee, J.Y., \& Podsakoff, N. P. (2003). Common method biases in behavioral research:A critical review of the literature and recommended remedies. Journal of applied psychology, 88(5), 879. doi: 10.1037/0021-9010.88.5.879

Polletta, F., \& Jasper, J. M. (200I). Collective identity and social movements. Annual Review of Sociology, 27, 283-305. doi: I0.1 146/annurev.soc.27.I.283

Preece, J., Nonnecke, B., \& Andrews, D. (2004). The top five reasons for lurking: improving community experiences for everyone. Computers in human behavior, 20(2), 201-223.doi: 10.1016/j.chb.2003.10.015

Pratte, G., Hurtubise, K., Rivard, L., Berbari, J. \& Camden, C. (2018). Developing a web platform to support a community of practice:A mixed methods study in pediatric physiotherapy. Journal of Continuing Education in the Health Professions, 38(I), 19-24. doi: 10.1097/ CEH.0000000000000185

Probst, G., \& Borzillo, S. (2008).Why communities of practice succeed and why they fail. European Management Journal, 26(5), 335-347. doi: I0.1016/j.emj.2008.05.003 
Purvis, R. L., Sambamurthy, V., \& Zmud, R.W. (200I).The Assimilation of Knowledge Platforms in Organizations. Organization Science, I 2(2), I I7-I35. doi: I0. I287/orsc. I 2.2. I I 7. I 0 I I5

Pyrko, I., Dörfler,V., \& Eden, C. (2017). Thinking together:What makes Communities of Practice work? Human Relations, 70(4), 389-409. doi: I0.I I 77/00 I87267I666I040

Qi, C., \& Chau, P.Y. K. (2018).Will enterprise social networking systems promote knowledge management and organizational learning ? An empirical study. Journal of Organizational Computing and Electronic Commerce, 28(I), 3 I-57. doi: I0.I080/I09 I9392.20 I8.I40708 I

Quinn, E. D., Cook, A., \& Rowland, C. (2019).An online community of practice to improve intervention for individuals with complex communication needs. Augmentative and Alternative Communication, 35(2), I42-I47. doi: I0.I080/074346I8.20I9.I566400

Quinn, R. E., \& Rohrbaugh, J. (1983).A Spatial Model of Effectiveness Criteria :Towards a Competing Values Approach to Organizational Analysis. Management Science, 29(3), 363377. doi: 10.1287/mnsc.29.3.363

Rajagopal, K., Joosten-Ten Brinke, D., Van Bruggen, J., \& Sloep, P. B. (20I2). Understanding personal learning networks:Their structure, content and the networking skills needed to optimally use them. First Monday, I 7(I), I-I2. doi: I0.52 I0/fm.v I 7il .3559

Rasula, J.,Vuksic, V. B., \& Stemberger, M. I. (20I2). The impact of knowledge management on organizational performance. Economic and Business Review, I4(2), I47-I68. doi: I0.78|3/2075-4I24.20I3/5-I/a. 18

Reinholt, M., Pedersen, T., \& Foss, N. J. (20I I).Why a central network position isn't enough: The role of motivation and ability for knowledge sharing in employee networks. Academy of Management Journal, 54(6), I277-I297. doi: I0.5465.amj.2009.0007

Reed, M.G., Godmaire, H., Abernethy, P. \& Guertin, M.A. (20I4). Building a community of practice for sustainability: Strengthening learning and collective action of Canadian biosphere reserves through a national partnership. Journal of Environmental Management, 145, 230-239. doi: I0.1016/j.jenvman.2014.06.030

Ren, Y., Harper, F. M., Drenner, S., Terveen, L., Kiesler, S., Kraut, R. E., ... Yuqing Ren Sara Drenner, Loren Terveen, Sara Kiesler, Robert E. Kraut, F. M. H. (20I2). Building Member Attachment in On-line Communities:Applying Theories of Group Identity and Interpersonal Bonds. MIS Quarterly, 36(3), 84I-864.

Ridings, C., Gefen, D., \& Arinze, B. (2006). Psychological Barriers : Lurker and Poster Motivation and Behavior in Online Communities. Communications of the Association for Information Systems, 18, 329-354. doi: 10.17705/ICAIS.01816

Ringle, C.,Wende, S., \& Will,A. (2005). SmartPLS 3.0. [Software]. Retrieved from www. smartpls.de

Robins, G., Elliott, P., \& Pattison, P. (200I). Network models for social selection processes. Social Networks, 23(I), I-30. doi: I0.I0I6/S0378-8733(0I)00029-6 
Robins, G., Pattison, P., \& Elliott, P. (200I). Network models for social influence processes. Psychometrika, 66(2), I6I-I89. doi: I0.1007/BF02294834

Rodman, L., \& Trespalacios, J. (2018). Knowledge-Sharing and Potential Virtual Communities of Practice in the US Coast Guard's Afloat Community: a Qualitative Pilot Study. TechTrends, 62(6), 602-6II.doi: |0.1007/s | |528-0|8-029|-8

Rothschild, M. (1999). Carrot sticks and promises: a conceptual framework for the management of public health and social issue behaviors. Journal of Marketing, 63(4), 24-37. doi: I0.2307//25 I 972

Rowe, M., Fernandez, M., Angeletou, S., \& Alani, H. (20I2). Community Analysis through Semantic Rules and Role Composition Derivation. Journal of Web Semantics, I8(I), 31 -47. doi: I0.1016/j. websem.20I2.05.002

Ruuska, I., \& Vartiainen, M. (2005). Characteristics of knowledge sharing communities in project organizations. International Journal of Project Management, 23(5), 374-379. doi: 10.10 I6/j. ijproman.2005.01.003

Ryan, J. (20I5). It ain't just what you do and the way that you do it: why discourse matters in higher education communities of practice. Higher Education Research and Development, 34(5), I00 I-I0I3. doi: 10.1080/07294360.2015.101 1087

Saarinen, S., Heimonen, T., Turunen, M., Mikkilä-erdmann, M., Raisamo, R., Erdmann, N., ... Keskinen, T. (20I5). Identifying User Interaction Patterns in E-Textbooks. The Scientific World Journal, 20I5, I-I2. doi: http://dx.doi.org/I0.II55/2015/98I520 Research

Safadi, H., \& Faraj, S. (20I4). Toward a Local Perspective on Online Collaboration. Collective Intelligence 20 /4, I-4. Retrieved from https://arxiv.org/pdf/ | 406.2977

Salanova, M., Peiró, J. M., \& Schaufeli,W. B. (2002). Self-efficacy specifity and burnout among information technology workers: An extension the job demand-control model. European Journal of Work and Organizational Psychology, I I (I), I-25. doi: I0. I080/I3594320 I43000735

Sarstedt, M., Ringle, C. M., Smith, D., Reams, R., \& Hair, J. F. (20I4). Partial least squares structural equation modeling (PLS-SEM):A useful tool for family business researchers. Journal of Family Business Strategy, 5(I), I05-II5. doi: 10.1016/j.jfbs.2014.01.002

Scarbrough, H. (2003). Knowledge management, HRM and the innovation process. International Journal of Manpower, 24(5), 50 I-5 I6. doi: I0.I I08/0 I4377203 I049I053

Schäfer, M.T., \& Van Es, K. F. (20I7). The datafied society: Studying culture through data. Amsterdam University Press. doi: 10.5 I I 7/9789462981 362

Scharkow, M. (2016). The Accuracy of Self-Reported Internet Use - A Validation Study Using Client Log Data The Accuracy of Self-Reported Internet Use - A Validation Study Using Client Log Data. Communication Methods and Measures, I (1), I3-27. doi: I0. I080/I 93 I 2458.20 I 5. I I I8446

Schein, E. H. (1985). Organizational culture and leadership. San Francisco: Jossey-Bass. 
Schepers, P., \& Van den Berg, P.T. (2007). Social Factors of Work-Environment Creativity. Journal of Business and Psychology, 2 I (3), 407-428. doi: I0.I007/s I 0869-006-9035-4

Schneckenberg, D. (2009).Web 2.0 and the empowerment of the knowledge worker. Journal of Knowledge Management, I3(6), 509-520. doi: I0.I I08/I36732709I0997I50

Schneider, B., Ehrhart, M. G., \& Macey,W. H. (20I3). Organizational Climate and Culture. Annual Review of Psychology, 64, 36 I-388. doi: I0.I I46/annurev-psych-I I 30 I I-I 43809

Schofield, K., Analoui, B., Brooks, J. \& Hussain, S.F. (2018). International Journal of Training \& Development, 22(3), 2 I0-22 I. doi: I0. I I I / ijtd. I 2129

Schwieger, D., Melcher, A., Ranganathan, C., \&Wen, J. H. (2004). Appropriating electronic billing systems:Adaptive structuration theory analysis. Human Systems Management, 23(4), 235-243. Retrieved from http://iospress.metapress.com/index/V9J43KPN5Q2PLRYV.pdf

Seibert, S. (20I5). The Meaning of a Healthcare Community of Practice. Nursing Forum, 50(2), 69-74. doi: I0.1 I I I/nuf.I2065

Senge, P. M. (1990). The fifth discipline:The art and practice of the learning organization. New York: Currency Doubleday.

Sessa,V. I., \& London, M. (20I5). Continuous learning in organizations: Individual, group, and organizational perspectives. Psychology Press.

Shih, H. P., \& Huang, E. (20I4). Influences of Web interactivity and social identity and bonds on the quality of online discussion in a virtual community. Information Systems Frontiers, I6(4), 627-64I. doi: 10.1007/s 10796-012-9376-7

Siemens, G. (2005). Connectivism: A Learning Theory for the Digital Age. International Journal of Instructional Technology \& Distance Learning, 2(I), I-8. Retrieved from http://itdl.org/journal/jan_05/ article0I.htm

Siemsen, E., Roth,A., \& Balasubramanian, S. (2008). How motivation, opportunity, and ability drive knowledge sharing:The constraining-factor model. Journal of Operations Management, 26(3), 426-445. doi: 10.1016/j.jom.2007.09.00I

Slater, S. F., \& Narver, J. C. (1995). Market Orientation and Learning Organization. Journal of Marketing, 59(3), 63. doi: $10.2307 / 1252120$

Slemp, G. R., Kern, M. L., \& Vella-Brodrick, D. (20I5).Workplace Well-Being:The Role of Job Crafting and Autonomy Support. Psychology of Well-Being, 5(I), I-I7. doi: I0. I I86/s I 36 I 2-0 I 5-0034-y

Smith, R. G., \& Farquhar,A. (2000). The road ahead for knowledge management. Al Magazine, 2 I (4), 17-40. doi: 10.1609/aimag.v21 i4.1528

Sonnenbichler,A. C. (20I0).A Community Membership Life Cycle Model. ArXiv, I006(427I), I-9. Retrieved from http://arxiv.org/abs/I006.427I 
Stephens, B., Chen,W., \& Butler, J. S. (2016). Bubbling up the good ideas: A two-mode network analysis of an intra-organizational idea challenge. Journal of Computer-Mediated Communication, 2 I (3), 2 I 0 229.doi: 10.1111 /jcc4.12158

Stieglitz, S., Meske, C., \& Schallenmuller, S. (20I3). Adoption of Social Media for Internal Usage in a Global Enterprise. In Proceedings of the 27th International Conference on Advanced Information Networking and Applications Workshops Adoption (pp. 1483-1488). doi: 10.1 I09/WAINA.2013.212

Stiglitz, J. E. (2015). Knowledge and Information. In Global Public Goods (pp. 306-325). New York: USA: Oxford university press.

Sukoco, B. M., \& Wu,W. (2010). The personal and social motivation of customers' participation in brand community. Journal of Business, 4(5), 6I4-622. Retrieved from http://www.academicjournals. org/app/webroot/article/article I3807| 3202_Sukoco and Wu.pdf

Sun, N., Rau, P. P. L., \& Ma, L. (20I4). Understanding lurkers in online communities:A literature review. Computers in Human Behavior, 38, I I0-I I7. doi: I0. I0 I6/j.chb.2014.05.022

Szulanski, G. (1995). Exploring internal stickiness: Impediments to the transfer of best practice within the firm. Strategic Management Journal, I 7(S2), 27-43. doi: I0.1002/smj.4250 I 71 I 05

Talò, C., Mannarini, T., \& Rochira,A. (20I4). Sense of Community and Community Participation:A Meta-Analytic Review. Social Indicators Research, I I 7(I), I-28. doi: I0.1007/s I I 205-0 I 3-0347-2

Thakadu, O.T., Irani, T.A., \& Telg, R. (20I3). Predictors of Knowledge-Sharing Behaviors Among Community-Based Natural Resources Organizations in the Okavango Delta, Botswana. Science Communication, 35(5), 572-602. doi: I0.II77/I0755470I247082 I

Theurer, C. P., Tumasjan, A., \& Welpe, I. M. (20I8). Contextual work design and employee innovative work behavior:When does autonomy matter? PLoS ONE, I3(I0), I-35. doi: I0.6084/ m9.figshare.4235 I86.Funding

Thompson,W. E., Hickey, J.V., \& Thompson, M. L. (2016). Society in focus:An introduction to sociology. Rowman \& Littlefield.

Tichy, N. M., Tushman, M. L., Fombrun, C., \& Tushman, M. L. ( 1979). Social Network Analysis For Organizations. The Academy of Management Review, 4(4), 507-5 I 9. doi: I0.5465/amr. 1979.4498309

Timans, W., Ahaus, K., van Solingen, R., Kumar, M., \& Antony, J. (2016). Implementation of continuous improvement based on Lean Six Sigma in small-and medium-sized enterprises. Total Quality Management \& Business Excellence. Total Quality Management \& Business Excellence, 27(3-4), 309-324. doi: I0.1080/I4783363.20I4.980 I 40

Tomasello, M. (20I4). A natural history of human thinking. Harvard University Press.

Tremblay, D. G., \& Psyché,V. (20I2). Analysis of processes of cooperation and knowledge sharing in a community of practice with a diversity of actors. Computer Science and Information Systems, 9(2), 917-94I. doi: I0.2298/CSISI I I 223020T 
Tseng, F. C., \& Kuo, F.Y. (20I4). A study of social participation and knowledge sharing in the teachers' online professional community of practice. Computers and Education, 72, 37-47. doi: I0.1016/j. compedu.2013.10.005

Tsvyk,V.A., \& Tsvyk, I.V. (2018). Individual Professionalization in Information Society: Challenges and Prospects. RUDN Journal of Sociology, I 8(3), 4 I 8-430. doi: I0.22363/23 I3-2272-20 I 8- I8-3-4 I 8-430

Turner, J. C., Hogg, M.A., Oakes, P. J., Reicher, S. D., \& Wetherell, M. S. (1 987). Rediscovering the social group:A self-categorization theory. Cambridge, MA, US: Basil Blackwell.

Turner, J. H. (1988). A theory of social interaction. Stanford University Press. doi: 10.2307/2579266

Turner, T., \& Pennington,W.W. (20I5). Organizational networks and the process of corporate entrepreneurship: how the motivation, opportunity, and ability to act affect firm knowledge, learning, and innovation. Small Business Economics, 45(2), 447-463. doi: I0.1007/s I I I87-0 I 5-9638-0

van den Hooff, B., \& de Leeuw van Weenen, F. (2004). Committed to share: commitment and CMC use as antecedents of knowledge sharing. Knowledge and Process Management, I I(I), I3-24. doi: 10.1002/kpm. 187

Van der Zee, H.T. M. (1995). De vijf denkparadigma's binnen het opleidings- en ontwikkelingsveld. Tijdschrift Voor Organisatiekunde En Sociaal Beleid, 2, I07-134.

Van Dijk, J. (20I2). The network society (3rd ed.). Sage Publications.

van Dijk, J.A. G. M. (2006). Digital divide research, achievements and shortcomings. Poetics, 34(4-5), 22I-235. doi: I0.10I6/j.poetic.2006.05.004

Van Dijk, J.A. G. M. (1997). The Reality of Virtual Communities. In Trends in Communication I (pp. 39-63).Amsterdam: Boom Publishers.

Van Maanen, J., \& Barley, S. R. (1984). Occupational communities: Culture and control in organizations. Research in Organizational Behavior, 6, 287-365. Retrieved from https://apps.dtic.mil/dtic/tr/fulltext/ u2/a | 22826.pdf

Van Meter, D. S., \& Van Horn, C. E. (1975). The policy implementation process:A conceptual framework. Administration \& Society, 6(4), 445-488. doi: I 0. I I 77/009539977500600404

Van Yperen, N.W.,Wörtler, B., \& De Jonge, K. M. M. (2016).Workers' intrinsic work motivation when job demands are high:The role of need for autonomy and perceived opportunity for blended working. Computers in Human Behavior, 60, 179-184. doi: I0.1016/j.chb.2016.02.068

Vaudel, M., Verheggen, K., Csordas, A., Raeder, H., Berven, F. S., Martens, L., ... Barsnes, H. (20I5). Exploring the potential of public proteomics data. Proteomics, I6(2), 2I4-225. doi: I0.1002/ pmic.20I500295

Venkatesh,V. (2000). "Determinants of perceived ease of use: Integrating control, intrinsic motivation, and emotion into the technology acceptance model". Information Systems Research, I I (4), 342-365. doi: I 0.I I 77/009539977500600404 
Venkatesh,V., Morris, M. G., Davis, G. B., \& Davis, F. D. (2003). User Acceptance of Information Technology: Toward a Unified View. MIS Quarterly, 27(3), 425-478. doi: 10.2307/30036540

Verburg, R. M., \& Andriessen, J. H. E. (2006). The assessment of communities of practice. Knowledge and Process Management, I3(I), I3-25. doi: I0.1002/kpm.24 I

Vollenbroek,W., \& De Vries, S. (2016). Interaction patterns in web-based knowledge communities: Two-mode network approach. In IC3K 20 I6 - Proceedings of the 8th International Joint Conference on Knowledge Discovery, Knowledge Engineering and Knowledge Management (Vol. 3, pp. I00-107). doi: I0.5220/0006035701000107

Vonderwell, S. (2003). An examination of asynchronous communication experiences and perspectives of students in an online course:A case study. The Internet and Higher Education, 6(I), 77-90. doi: I0.10I6/SI096-75I6(02)00I64-I

Vries, S. De. (2003). Online knowledge communities : meeting places for continuing professional development. In Informatiics and the Digital Society (pp. 215-224). Boston, MA: Springer. doi: |0.1007/978-0-387-35663-|

Wang, G. Robins, P. P. (2009). PNet: program for the simulation and estimation of exponential random graph models [Software]. Retreived from http://www.melnet.org.au/pnet

Wang, P., Pattison, P., \& Robins, G. (2013). Exponential random graph model specifications for bipartite networks-A dependence hierarchy. Social Networks, 35. doi: I0.1016/j.socnet.201 I.12.004

Wang,Y., \& Fesenmaier, D. R. (2004). Towards understanding members' general participation in and active contribution to an online travel community. Tourism Management, 25(6), 709-722. doi: I0.1016/j.tourman.2003.09.0I I

Wasko, M. L., \& Faraj, S. (2005).Why Should I Share? Examining Social Capital and Knowledge Contribution in Electronic Networks of Practice. MIS Quarterly, 29(I), 35-56. doi: $10.2307 / 25 \mid 48667$

Wasserman, S., \& Faust, K. (1994). Social network analysis: Methods and applications. Cambridge University Press, 8.

Webster-Wright, A. (2009). Reframing Professional Development Through Understanding Authentic Professional Learning. Review of Educational Research, 79(2), 702-739. doi: I0.3 I02/0034654308330970

Weick, K. E. (1979). The Social Psychology of Organizing (2nd ed.).Addison-Wesley, Reading.

Wellman, B., \& Berkowitz, S. D. (1988). Social structures:A network approach. Press Syndicate of the University of Cambridge.

Wellman, B. (1997). An electronic group is virtually a social network. Culture of the Internet, 4, 179-205. Retrieved from http://www.ai.soc.i.kyoto-u.ac.jp/ ishida/sisys/sisys200 I/electronicgroup.pdf

Wenger, E., Trayner, B. \& De Laat, M. (20I I). Promoting and assessing value creation in communities 
and networks: a conceptual framework. Ruud de Moor Centrum, I8.

Wenger \& Snyder,W. M. (2000). Communities of Practice:The Organizational Frontier. Harvard Business Review, 24(3), 383-40I. doi: I0.1 I77/0I70840603024003909

Wenger, B. E. (2004). Knowledge management as a doughnut : Shaping your knowledge strategy through communities of practice Knowledge management as a doughnut : Shaping your knowledge strategy through communities of practice. Ivey Business Journal, 68(5 I9), I-9. Retrieved from http:// search.ebscohost.com/login.aspx?direct=true\&db=bth\&AN= 28287 I 5 \&site=ehost-live

Wenger, E. (1998). Communities of practice: Learning as a social system. Systems Thinker, 9(5), I-I0.

Wenger, E. (1999). Communities of Practice: Learning, Meaning, and Identity. Cambridge: Press Syndicate of the University of Cambridge.

Wenger, E. (2000). Communities of Practice and Social Learning Systems. Organization, 7(2), 225-246. doi: I0.1 I77//35050840072002

Wenger, E. (2006). Communities of practice in and across 2 Ist -century organizations. Communities, I-9.

Wenger, E. (1998). Communities of practice: Learning as a social system. Systems thinker, 9(5), 2-3.

Wenger, E., McDermott, R.A., \& Snyder,W. (2002). Cultivating communities of practice:A guide to managing knowledge. Harvard Business Press.

Wenger, E.,White, N., Smith, J. D., \& Rowe, K. (2005). Technology for communities. In CEFRIO (PP. I-I5). Retrieved from http://waterwiki.net/images/9/97/Technology_for_communities_-_book_ chapter.pdf

Wick, C. (2000). Knowledge management and leadership opportunities for technical communicators. Technical Communication, 47(4), 515-529. Retrieved from http://citeseerx.ist.psu.edu/viewdoc/ download?doi=|0.I.I.47| .| $893 \&$ rep=rep I\&type=pdf

Wood, M. S. (2005). Determinants of Shared Leadership in Management Teams. International Journal of Leadership Studies, I(I), 64-85. Retrieved from https://www.regent.edu/acad/global/publications/ijls/ new/vol I iss I/wood/determinants.pdf

Wood, R., \& Bandura,A. (1989). Social Cognitive Theory of Organizational Management. The Academy of Management Review, I4(3), 36 I-384. doi: http://dx.doi.org/I0.2307/258I 73

Xu,W.W., Chiu, I.H., Chen,Y. \& Mukherjee,T. (20I5). Twitter hashtags for health:Applying network and content analyses to understand the health knowledge sharing in a Twitter-based community of practice. Quality and Quantity, 49(4), | 36 I- | 380. http://dx.doi.org/ | 0. I007/s I I |35-0 | 4-005 I-6

Xu, J., Quaddus, M., \& Gao, X. (20I4). Towards a Knowledge Sharing Model for Small Businesses. The International Technology Management Review, 4(I), I2. doi: I0.2991/itmr.20 I4.4.1.2

Young, C. (20I3). Community management that works: How to build and sustain a thriving online 
health community. Journal of Medical Internet Research, I5(6). doi: I0.2196/jmir.250 I

Zaheer,A., \& Bell, G. G. (2005). Benefiting from network position: Firm capabilities, structural holes, and performance. Strategic Management Journal, 26(9), 809-825. doi: 10.1002/smj.482

Zappa, P., \& Lomi,A. (2016). Knowledge sharing in organizations:A multilevel network analysis. In Multilevel network analysis for the social sciences (pp. 333-353). Springer. doi: 10.1007/978-3-3 I924520-I_14

Zboralski, K., Salomo, S., \& Gemuenden, H. (2006). Organizational benefits of communities of practice:A two-stage information processing model. Cybernetics and Systems, 37(6), 533-552. doi: |0.1080/0 | 96972060073446 |

Zhang,W., \&Watts, S. (20I5). Online communities as communities of practice : a case study. Journal of Knowledge Management, I2(4), 55-7I. doi: I0.I I08/I36732708I0884255

Zhu, H., Kraut, R., \& Kittur,A. (20I2). Effectiveness of shared leadership in online communities. In Proceedings of the ACM 2012 Conference on Computer Supported Cooperative Work, 407-4I6. doi: |0.| | 45/2 | 45204.2 | 45269 


\section{Appendices}

Appendix 2.I. List of CoP definitions

\section{Definition}

I “A group of people engaging in a common activity in which there are established sociocultural norms of interaction"

2 "A group of people "who share a concern, a set of problems, or a passion about a topic, and who deepen their knowledge and expertise in this area by interacting on an ongoing basis"

3 "Commonly function strategically within and across existing networks as virtual CoPs that practitioners join or as practice learning environments, where identification around shared learning takes place"

4 "A set of relations among persons, activity, and world, overtime and in relation with other tangential and overlapping communities of practice. A community of practice is an intrinsic condition for the existence of knowledge (...) It does imply participation in an activity system about which participants share understandings concerning what they are doing and what that means in their lives and for their communities"

\section{Author(s) Cited by}

Couper, Denny \&

Watkins (2016, p. 5).

Wenger, McDermott, Johnston (2017, p.

\& Snyder, (2002) 669).

Mayrhofer, Goodman

\& Holman (2015, $p$.

260).

Wenger (1991). Gonçalves, Nogueira,Vieira da Silva (20I6, p. II0). 


\section{Definition \\ $5 \quad$ "A persistent, sustaining social network of individuals who share and develop an overlapping knowledge base, set of beliefs, values, history and experiences focused on a common practice and/or mutual enterprise"}

6 "A group of people who share a concern or passion for something they do and learn how to do it better as they interact regularly." The expression "virtual communities of practices" ( $v$ CoPs) is applied when members use information technologies (eg, web forums, internet-based chats or electronic sharing of resources) as their primary method of interaction"

7 "Learning communities in which members are geographically separated and communicate primarily through either synchronous or asynchronous virtual forums"

8 "Groups of people who share a concern, a set of problems, or a passion about a topic, and who deepen their knowledge and expertise in this area by interacting on an ongoing basis"

9 "An informal group in which members share issues of common concern, develop a shared working style, and complete certain specific actions"

I0 "Educational community where learning takes place through the practices and meanings that are developed, shaped, and negotiated among its members"

II "a socially situated, practice-based approach to learning, in which the learning that takes place is viewed in terms of a series of collective, relational and social processes (as opposed to an individual, solitary process of knowledge acquisition)"

\section{Author(s) \\ Cited by}

Barab et al. (2003).

Cruess, Cruess \& Steinert (2018, p. 186)

Wenger (2002); Dubé Pratte, Hurtubise, et al. (2005); Bourhis et al. (2005) Rivard, Berbari \& Camden (2018, p.

19)

Dubé et al. (2005), Rodman \&

Liao (2017) \&Wenger Trespalacios (2018, et al. (2002). p. 602).

Wenger et al. (2002, p. 4).

Kensington-Miller (20I8, p. 68I).

Hsu, Lin, Fang \& Liu (2018, p. 5).

Aguilar and Krasny (20II) and Wenger (1998).

Ceaser (2018, p. 46I).

Lave \&Wenger (199I) Lewis \& Kelly (2018, p. 3). 
12 "A group of individuals informally bound together by shared expertise and passion for a joint enterprise'. This shared expertise is bound within a specific, in this case 'workplace', context with individuals contributing to a shared aim. Learning, in this forum, evolves naturally and there are group benefits and purposes to membership of the community as a whole"

I3 "An online community for like-minded individuals in geographically dis- persed areas. A VCoP is an emergent online community where professionals in an industry share and seek knowledge related to their practice. In accordance with the characteristics of a conventional community of practice, it is a place where a rich shared repertoire community identity (i.e., common interest, theme, organisation) and individual member identity (i.e., moderators, experts) are developed through social interactions"

14 "A group of people who 'deepen their knowledge and expertise in [an] area by interacting with one another on an on-going basis"

I5 "A group of people who share an interest or a passion for something that they practice, and who learn how to do it better through regular interaction"

16 "A set of relations among per- sons, activity, and world, over time and in relation with other tangential and overlapping communities of practice"

17 "Collective learning networks formed around shared challenges"
Wenger and Snyder (2000, p. 139) \&

(Shipton, 2006)

Schofield, Analoui, Brooks, Hussain (20I8, p. $21 \mathrm{I})$.

Pan et al. (2015, p. 62)

Wenger, McDermott, and Snyder (2002)

Goodyear \& Casey (20I5, p. 188).

Wenger (1998)

Cundill, Roux, Parker (20I5, p. 2).

Lave \&Wenger (199I) Gilbuena, et al (2015, p. 10).

Lave and Wenger, (I99I)

Kalafatis, Lemos, Lo \& Frank (20I5, p. 3I). 


\section{Definition}

I8 "A group of people who interact, learn together, build relationships, and in the process develop a sense of belonging and mutual commitment"

19 "An informal learning community characterized by a shared practice of its members, their voluntary engagement and a shared repertoire of communal resources (routines, norms, artifacts, vocabulary, styles, etc.) that members have developed over time"

20 "Group of people who share a craft or profession; it signifies a way of knowing and learning and focuses on people engaged in sharing knowledge and practices regarding a specific set of issues"

$21 \quad$ "A group of people who share concern or passion for what they do and who learn how to do it better through regular interaction. Its members share a common do- main of interest, and the CoP renews itself by generating new knowledge"

22 "Ways of combining and integrating language, actions, interactions, ways of thinking, believing, valuing, and using various symbols, tools, and objects to enact a particular sort of socially recognizable identity"

23 "Shared linguistic and social norms, values, and patterns of activity"
Anderson (2007)

Lave and Wenger (I99I) and Wenger et al. (2009).

Lave \&Wenger, (I99|).

\section{Cited by}

Hou (2015, p. 7).

and Snyder (2002)

Lave \&Wenger (199I) Dolinska \&

D'Aquino (2016, p. 123).

Mohtar \& Lawford (2016, p.196).

Wenger and Snyder

Mohtar \& Lawford (2016, p. 196).

Correa, Martínez-

Arbelaiz \&

Aberasturi-Apraiz (20I5, p. 68).

González-Howard \& McNeill (2016, p. 530).

$\mathrm{Xu}$, Chiu, Chen \& Mukherjee (20I5, p. I362).

Goodyear, Casey, Kirk (20I4, p. 929). 


Definition

26 "People learn socially from their peers"

27 "Groups of people who share a concern or a passion for something they do and learning how to do it better as they interact regularly"

28 "Virtual < communities of practice (VCoPs) > have been proposed as a strategy for reducing isolation by overcoming barriers to knowledge sharing within a COP by augmenting face-to-face communication and facilitating collaboration online, particularly through social media technologies"

29 "A collection of individuals sharing mutually defined practices, beliefs, and understandings over a period of time, in the pursuit of a shared endeavor"

30 "Social groups bound by a common concern or passion and a desire to learn how to improve their practice"

31 "A joint enterprise that seeks to address gaps in knowledge; be organized through mutual engagement and reciprocity that involves sharing, interacting, and supporting one another; and developing a shared repertoire of artifacts (e.g., routines, words, tools, etc.) produced by the community"

32 "Groups of people bound by a shared practice who interact and learn from each other"

33 "Designed to systematically leverage the skills and passions of individual administrative and clinical practitioners across institutions toward common goals"
Author(s)

Wenger (1998)

Wenger and Snyder (2000)

Cited by

Nykvist (20l4, p. 437).

Barnett et al. (20l4, p. 2).

Barnett et al. (20l4, p.3).

Wenger (1998)

Evans et al (2014, p. 2 I5).

Wenger (2003)

Reed et al. (2014, p. 23 I).

Wenger (1998)

Reed et al. (2014, p. 23I).

Wenger (1998)

Dennen (20I4, p. 35I).

Fung et al. (20I4, p.

28) 


\begin{tabular}{|c|c|c|c|}
\hline & Definition & Author(s) & Cited by \\
\hline 34 & $\begin{array}{l}\text { "Groups of people who share a } \\
\text { concern, a set of problems, and a } \\
\text { passion about a topic, and who deepen } \\
\text { their knowledge and expertise in this } \\
\text { area by interacting on an ongoing basis" }\end{array}$ & $\begin{array}{l}\text { (Wenger, McDermott, } \\
\text { \& Snyder, 2002, p. 4) }\end{array}$ & $\begin{array}{l}\text { Seibert }(2015, \mathrm{p} . \\
70) \text {. }\end{array}$ \\
\hline 35 & $\begin{array}{l}\text { "Promotes a dynamic social } \\
\text { participative approach to learning and } \\
\text { discovery" }\end{array}$ & $\begin{array}{l}\text { Andrew and Ferguson } \\
(2008)\end{array}$ & $\begin{array}{l}\text { Seibert }(2015, \mathrm{p} . \\
70) \text {. }\end{array}$ \\
\hline 36 & $\begin{array}{l}\text { "An integrated approach to learning } \\
\text { achieved through a combination of } \\
\text { social engagement and collaborative } \\
\text { working in an authentic practice } \\
\text { environment" }\end{array}$ & $\begin{array}{l}\text { Andrew and Ferguson } \\
(2008)\end{array}$ & $\begin{array}{l}\text { Seibert }(2015, \mathrm{p} . \\
70) \text {. }\end{array}$ \\
\hline 37 & $\begin{array}{l}\text { "Practical and social support and } \\
\text { learning to facilitate improvement } \\
\text { activity" }\end{array}$ & $\begin{array}{l}\text { Chandler and Fry } \\
\text { (2009) }\end{array}$ & $\begin{array}{l}\text { Seibert }(2015, \mathrm{p} . \\
70) \text {. }\end{array}$ \\
\hline 38 & $\begin{array}{l}\text { "Shared learning and teaching, collegial } \\
\text { relationships, nonhierarchical structures } \\
\text { and commitment to change" }\end{array}$ & White et al. (2008) & $\begin{array}{l}\text { Seibert }(2015, \mathrm{p} . \\
70) \text {. }\end{array}$ \\
\hline 39 & $\begin{array}{l}\text { "Learning that focuses on networks of } \\
\text { connections" }\end{array}$ & $\begin{array}{l}\text { (Cormier \& Siemens, } \\
\text { 2010; Downes, 20I2; } \\
\text { Siemens \& Matheos, } \\
2010 \text { ) }\end{array}$ & $\begin{array}{l}\text { Guerin, Carter \& } \\
\text { Aitchison (20I5, p. } \\
2 \mid 3) \text {. }\end{array}$ \\
\hline 40 & $\begin{array}{l}\text { "Groups of people who share a } \\
\text { concern, a set of problems, or a passion } \\
\text { about a topic, and who deepen their } \\
\text { knowledge and expertise in this area by } \\
\text { interacting on an ongoing basis" }\end{array}$ & $\begin{array}{l}\text { (Wenger, McDermott, } \\
\text { \& Snyder, 2002, p. 4) }\end{array}$ & $\begin{array}{l}\text { Gannon \& } \\
\text { Prothero (2018, p. } \\
\text { 592). }\end{array}$ \\
\hline 41 & $\begin{array}{l}\text { "Communities of practice are groups } \\
\text { of people who share a concern, a set } \\
\text { of problems, or a passion about a topic, } \\
\text { and who deepen their knowledge and } \\
\text { expertise in this area by interacting on } \\
\text { an ongoing basis" }\end{array}$ & $\begin{array}{l}\text { (Wenger, McDermott, } \\
\text { \& Snyder, 2002, p. 4) }\end{array}$ & $\begin{array}{l}\text { Baker \& Beames } \\
(2016, \text { p. } 73)\end{array}$ \\
\hline 42 & $\begin{array}{l}\text { "A set of relations among persons, } \\
\text { activity and world' in which people are } \\
\text { bound together by shared expertise and } \\
\text { passion for a joint enterprise or goal" }\end{array}$ & $\begin{array}{l}\text { (Wenger \& Snyder, } \\
\text { 2000) }\end{array}$ & $\begin{array}{l}\text { Tseng \& Kuo, } \\
(2014, \text { p. } 37)\end{array}$ \\
\hline
\end{tabular}


Appendix 6.I: Items in the survey focused on the individual perspective

Items belonging to the levels of social behaviour
I
2
3
4
5
6

Never

Less frequent than once a

Multiple

Once a day

Multiple

times a week

times a day

week

$\begin{array}{lll}\text { \# } & \begin{array}{l}\text { English } \\ \text { translation of } \\ \text { the items: }\end{array}\end{array}$

\section{Adapted from \\ or based on \\ scales or \\ findings from:}

P5

\begin{tabular}{|c|c|c|}
\hline $\begin{array}{l}\text { I read, watch } \\
\text { or listen to the } \\
\text { information that } \\
\text { others share }\end{array}$ & $\begin{array}{l}\text { Ik lees, bekijk } \\
\text { of beluister de } \\
\text { informatie die } \\
\text { anderen delen }\end{array}$ & $\begin{array}{l}\text { Knowledge } \\
\text { acquisition } \\
\text { behaviour }\end{array}$ \\
\hline
\end{tabular}

Millen et al., (2002)

P7

$\begin{array}{ll}\text { I share } & \text { Ik deel } \\ \text { information } & \text { informatie dat } \\ \text { that is / can } & \text { voor collega's } \\ \text { be relevant } & \text { binnen mijn } \\ \text { for colleagues } & \text { eigen organisa } \\ \text { within my own } & \text { relevant is/kan } \\ \text { organisation. } & \text { zijn. Dit kan zi, } \\ \text { This may include: } & \text { (werk)rapport } \\ \text { (work) reports } & \text { en/of officiële } \\ \text { and / or official } & \text { documenten, } \\ \text { documents, } & \text { methodiek/ } \\ \text { methodology } & \text { methoden, } \\ \text { / methods, } & \text { (praktische) } \\ \text { (practical) } & \text { kennis en/ } \\ \text { knowledge and } & \text { of (werk) } \\ \text { / or (work) } & \text { ervaringen } \\ \text { experiences } & \end{array}$

P8

$\begin{array}{lll}\text { I appreciate the } & \text { Ik waardeer de } & \text { Social } \\ \text { contributions of } & \text { bijdragen van } & \text { interaction } \\ \text { colleagues. This } & \text { collega's. Dit kan } & \text { behaviour } \\ \text { can be with a } & \text { zijn: met een } & \\ \text { like, thanks, etc. } & \text { like, dank, etc. } & \end{array}$

Social interaction behaviour

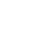




\begin{tabular}{|c|c|c|c|c|}
\hline \# & $\begin{array}{l}\text { English } \\
\text { translation of } \\
\text { the items: }\end{array}$ & $\begin{array}{l}\text { Original items } \\
\text { in Dutch: }\end{array}$ & Concept: & $\begin{array}{l}\text { Adapted from } \\
\text { or based on } \\
\text { scales or } \\
\text { findings from: }\end{array}$ \\
\hline P9 & $\begin{array}{l}\text { I get to know } \\
\text { (new) colleagues } \\
\text { within my } \\
\text { organisation }\end{array}$ & $\begin{array}{l}\text { Ik leer (nieuwe) } \\
\text { collega's binnen } \\
\text { mijn organisatie } \\
\text { kennen }\end{array}$ & $\begin{array}{l}\text { Social } \\
\text { interaction } \\
\text { behaviour }\end{array}$ & $\begin{array}{l}\text { Chiu et al. } \\
\text { (2006); Ruuska, } \\
\text { l., \& Vartiainen } \\
\text { (2005) }\end{array}$ \\
\hline PIO & $\begin{array}{l}\text { I communicate } \\
\text { with colleagues } \\
\text { within my } \\
\text { organisation. } \\
\text { This can be } \\
\text { participation } \\
\text { in discussions, } \\
\text { making } \\
\text { appointments, } \\
\text { etc. }\end{array}$ & $\begin{array}{l}\text { Ik communiceer } \\
\text { met collega's } \\
\text { binnen mijn } \\
\text { organisatie. } \\
\text { Dit kan zijn: } \\
\text { deelname in } \\
\text { discussies, } \\
\text { maken van } \\
\text { afspraken, etc. }\end{array}$ & $\begin{array}{l}\text { Social } \\
\text { interaction } \\
\text { behaviour }\end{array}$ & Context-based \\
\hline P6 & $\begin{array}{l}\text { I ask colleagues } \\
\text { within my } \\
\text { organisation } \\
\text { for information } \\
\text { relevant to } \\
\text { me. This can } \\
\text { be: (work) } \\
\text { reports, official } \\
\text { documents, } \\
\text { methodology } \\
\text { / methods, } \\
\text { (practical) } \\
\text { knowledge and } \\
\text { experiences }\end{array}$ & $\begin{array}{l}\text { Ik vraag collega's } \\
\text { binnen mijn } \\
\text { organisatie } \\
\text { naar voor } \\
\text { mij relevante } \\
\text { informatie. Dit } \\
\text { kan zijn: (werk) } \\
\text { rapporten, } \\
\text { officiële } \\
\text { documenten, } \\
\text { methodiek/ } \\
\text { methoden, } \\
\text { (praktische) } \\
\text { kennis en } \\
\text { ervaringen }\end{array}$ & $\begin{array}{l}\text { Social } \\
\text { interaction } \\
\text { behaviour }\end{array}$ & $\begin{array}{l}\text { van den Hooff } \\
\& \text { de Leeuw van } \\
\text { Weenen }(2004)\end{array}$ \\
\hline PII & $\begin{array}{l}\text { I read the } \\
\text { newsletter with } \\
\text { recent activities, } \\
\text { which I receive } \\
\text { periodically }\end{array}$ & $\begin{array}{l}\text { lk lees de } \\
\text { samenvattende } \\
\text { mail met recente } \\
\text { activiteiten, die } \\
\text { ik periodiek } \\
\text { ontvang }\end{array}$ & $\begin{array}{l}\text { Knowledge } \\
\text { acquisition } \\
\text { behaviour }\end{array}$ & Context-based \\
\hline
\end{tabular}


Items belonging to the Motivation

\begin{tabular}{l|l|l|l|l|l|l}
$\mathbf{I}$ & $\mathbf{2}$ & $\mathbf{3}$ & $\mathbf{4}$ & $\mathbf{5}$ & $\mathbf{6}$ & $\mathbf{7}$ \\
\hline $\begin{array}{l}\text { Strongly } \\
\text { disagree }\end{array}$ & Disagree & $\begin{array}{l}\text { More } \\
\text { or less } \\
\text { disagree }\end{array}$ & Undecided & $\begin{array}{l}\text { More or } \\
\text { less agree }\end{array}$ & Agree & $\begin{array}{l}\text { Strongly } \\
\text { agree }\end{array}$
\end{tabular}

\begin{tabular}{llll}
\hline English & $\begin{array}{l}\text { Original items } \\
\text { translation of } \\
\text { the items: }\end{array}$ & & $\begin{array}{l}\text { Adapted from } \\
\text { in Dutch: }\end{array}$ \\
& & $\begin{array}{l}\text { or based on } \\
\text { scales or } \\
\text { findings from: }\end{array}$ \\
\hline
\end{tabular}

$\begin{array}{llll}\text { MI } & \begin{array}{l}\text { To be informed } \\ \text { of the latest } \\ \text { developments } \\ \text { within my area } \\ \text { of expertise }\end{array} & \begin{array}{l}\text { Om op de } \\ \text { hoogte te zijn } \\ \text { van de laatste } \\ \text { ontwikkelingen } \\ \text { binnen het } \\ \text { vakgebied }\end{array} & \text { Lee \& Ma (20I2) } \\ \text { M2 } & \begin{array}{ll}\text { To be informed } \\ \text { of the latest } \\ \text { developments } \\ \text { within the } \\ \text { organisation }\end{array} & \begin{array}{l}\text { Om op de } \\ \text { hoogte te zijn } \\ \text { van de laatste } \\ \text { ontwikkelingen } \\ \text { binnen de } \\ \text { organisatie }\end{array} & \text { Lee \& Ma (20I2) } \\ \end{array}$

M3

To know which activities are

Om te

Informing

Lee \& Ma (20I2) planned weten welke activiteiten er op de planning staan

M4

To search for

Om naar information that informatie te is relevant to my zoeken die work relevant is voor het uitvoeren van mijn werk

Informing

Lee \& Ma (20I2)

M5

To find information

Om informatie Informing op een later later moment terug te vinden others do

Om te zien wat Informing anderen doen

Leonardi (2014) 


\begin{tabular}{llll}
\hline$\#$ & $\begin{array}{l}\text { English } \\
\text { translation of } \\
\text { the items: }\end{array}$ & $\begin{array}{l}\text { Original items } \\
\text { in Dutch: }\end{array}$ & $\begin{array}{l}\text { Adapted from } \\
\text { or based on } \\
\text { scales or } \\
\text { findings from: }\end{array}$ \\
\hline
\end{tabular}

M7

M8

M9

MIO

MI I

MI2

MI3

MI4

MI5
To share

knowledge, insights and experiences

To contribute in subject-related discussions

To contribute online in projects

To get support from other professionals

To get in touch with other professionals
Om ervaringen, kennis en inzichten te delen

Om bij te dragen in vakinhoudelijke discussies

Om online bij te dragen in

Om ondersteuning van andere professionals te krijgen

Om in contact te komen met andere professionals

Om in contact te blijven met andere with other professionals professionals projecten with other professionals

To present myself

To continuously improve my work practices
Om ideeën te delen met andere professionals

Om mij te profileren
Work performance performance

Work performance

Work performance

Work performance

Work performance

Work performance

Kankanhalli et al. (2005)

Xu, Quaddus, \&

Gao (20I4)

Context-driven

(Xu, Quaddus, \&

Gao, 20I4)

Han et al. (2007)

Lee \& $\mathrm{Ma}$

(20|2); Bock et

al. (2005)

Lee \& Ma

(20I2); Bock

et al. (2005);

Deursen et al.

(20II)

Lee \& Ma (20I2); Bock et al. (2005);

Deursen et al.

(20II)

Lee \& $\mathrm{Ma}$

(20I2); Bock et

al. $(2005$

Social reputation Lee \& Ma (20I2)

Work

Xu, Quaddus, \&

performance

Gao (20|4) 


\begin{tabular}{|c|c|c|c|c|}
\hline \# & $\begin{array}{l}\text { English } \\
\text { translation of } \\
\text { the items: }\end{array}$ & $\begin{array}{l}\text { Original items } \\
\text { in Dutch: }\end{array}$ & Concept: & $\begin{array}{l}\text { Adapted from } \\
\text { or based on } \\
\text { scales or } \\
\text { findings from: }\end{array}$ \\
\hline MI6 & $\begin{array}{l}\text { Because it } \\
\text { clearly has } \\
\text { added value to } \\
\text { perform my job }\end{array}$ & $\begin{array}{l}\text { Omdat het } \\
\text { duidelijk } \\
\text { meerwaarde } \\
\text { heeft voor het } \\
\text { uitvoeren van } \\
\text { mijn werk }\end{array}$ & $\begin{array}{l}\text { Work } \\
\text { performance }\end{array}$ & Context-based \\
\hline MI7 & $\begin{array}{l}\text { To perform } \\
\text { (part of) } \\
\text { my work } \\
\text { independently of } \\
\text { place }\end{array}$ & $\begin{array}{l}\text { Om (een deel } \\
\text { van) mijn werk } \\
\text { onafhankelijk } \\
\text { van plaats uit te } \\
\text { voeren }\end{array}$ & $\begin{array}{l}\text { Work } \\
\text { performance }\end{array}$ & $\begin{array}{l}\text { Kulkarni et al. } \\
\text { (2007) }\end{array}$ \\
\hline MI8 & $\begin{array}{l}\text { To perform } \\
\text { (part of) } \\
\text { my work } \\
\text { independently of } \\
\text { time }\end{array}$ & $\begin{array}{l}\text { Om (een deel } \\
\text { van) mijn werk } \\
\text { onafhankelijk } \\
\text { van tijd uit te } \\
\text { voeren }\end{array}$ & $\begin{array}{l}\text { Work } \\
\text { performance }\end{array}$ & $\begin{array}{l}\text { Kulkarni et al. } \\
\text { (2007) }\end{array}$ \\
\hline MI9 & $\begin{array}{l}\text { Because I feel } \\
\text { pressure from } \\
\text { the organisation } \\
\text { to participate }\end{array}$ & $\begin{array}{l}\text { Omdat ik vanuit } \\
\text { de organisatie } \\
\text { druk voel om } \\
\text { deel te nemen }\end{array}$ & Subjective norm & $\begin{array}{l}\text { Hung, Lai, \& } \\
\text { Chou, (20I5); } \\
\text { Scarbrough } \\
\text { (2003) }\end{array}$ \\
\hline M20 & $\begin{array}{l}\text { Because I feel } \\
\text { pressure from } \\
\text { my colleagues to } \\
\text { participate }\end{array}$ & $\begin{array}{l}\text { Omdat ik vanuit } \\
\text { mijn collega's } \\
\text { druk voel om } \\
\text { deel te nemen }\end{array}$ & Subjective norm & $\begin{array}{l}\text { Hung et al. } \\
\text { (20I5); Lewis et } \\
\text { al., (2003) }\end{array}$ \\
\hline
\end{tabular}


Items belonging to the Ability

\begin{tabular}{l|l|l|l|l|l|l}
$\mathbf{I}$ & $\mathbf{2}$ & $\mathbf{3}$ & $\mathbf{4}$ & $\mathbf{5}$ & $\mathbf{6}$ & $\mathbf{7}$ \\
\hline $\begin{array}{l}\text { Strongly } \\
\text { disagree }\end{array}$ & Disagree & $\begin{array}{l}\text { More } \\
\text { or less } \\
\text { disagree }\end{array}$ & Undecided & $\begin{array}{l}\text { More or } \\
\text { less agree }\end{array}$ & Agree & $\begin{array}{l}\text { Strongly } \\
\text { agree }\end{array}$
\end{tabular}

\begin{tabular}{llll} 
\# & $\begin{array}{l}\text { English } \\
\text { translation of } \\
\text { the items: }\end{array}$ & $\begin{array}{l}\text { Original items } \\
\text { in Dutch: }\end{array}$ & $\begin{array}{l}\text { Adapted from } \\
\text { or based on } \\
\text { scales or } \\
\text { findings from: }\end{array}$ \\
\hline
\end{tabular}

Al

I am able Ik ben in staat Ability

Siemsen et al

to share my

om mijn kennis,

(2008)

knowledge,

ervaringen

experiences and

en inzichten

insights with

te delen met

others

anderen

$\mathrm{A} 2$

I am able

Ik ben in staat

Ability

Turner \&

to answer

om vragen te

Pennington

questions,

beantwoorden,

(20I5)

give advice

advies te geven

or provide

of voorbeelden

examples for

te verstrekken

questions or

bij vragen of

requests from

verzoeken van

others

anderen

A3

I am able to

Ik ben in staat

Ability

Turner \&

express myself

om me goed

well in words

uit te drukken

Pennington

in woord en

(2015)

geschrift

A4

I am able to

Ik ben in staat

Ability

Turner \&

respond sensibly

om inhoudelijk

to messages

zinvol te

Pennington

from others

reageren op

(20I5)

berichten van

anderen 


\begin{tabular}{lll}
\hline$\# \quad \begin{array}{l}\text { English } \\
\text { translation of } \\
\text { the items: }\end{array}$ & $\begin{array}{l}\text { Original items Concept: } \\
\text { in Dutch: }\end{array}$
\end{tabular}

\begin{tabular}{|c|c|c|c|c|}
\hline & & & & findings from: \\
\hline A5 & $\begin{array}{l}\text { I have no } \\
\text { difficulty } \\
\text { to contact } \\
\text { colleagues who } \\
\text { are known to } \\
\text { me, directly via } \\
\text { internet }\end{array}$ & $\begin{array}{l}\text { Ik heb er geen } \\
\text { moeite mee om } \\
\text { online contact te } \\
\text { leggen met voor } \\
\text { mij bekende } \\
\text { collega's }\end{array}$ & Ability & $\begin{array}{l}\text { Cadiz et al. } \\
(2009)\end{array}$ \\
\hline A6 & $\begin{array}{l}\text { I have no } \\
\text { difficulty } \\
\text { to contact } \\
\text { colleagues who } \\
\text { are unknown to } \\
\text { me, directly via } \\
\text { internet }\end{array}$ & $\begin{array}{l}\text { Ik heb er geen } \\
\text { moeite mee om } \\
\text { online contact te } \\
\text { leggen met voor } \\
\text { mij onbekende } \\
\text { collega's }\end{array}$ & Ability & $\begin{array}{l}\text { Cadiz et al. } \\
(2009)\end{array}$ \\
\hline A7 & $\begin{array}{l}\text { I have sufficient } \\
\text { IT skills to } \\
\text { connect online } \\
\text { with colleagues } \\
\text { within } \\
<\text { CoP of the } \\
\text { organisation> }\end{array}$ & $\begin{array}{l}\text { lk heb } \\
\text { voldoende ICT- } \\
\text { vaardigheden } \\
\text { om online } \\
\text { contact te } \\
\text { leggen met } \\
\text { collega's binnen } \\
\text { <CoP van de } \\
\text { organisatie> }\end{array}$ & Ability & $\begin{array}{l}\text { Deursen et al., } \\
(20 \mathrm{II})\end{array}$ \\
\hline A8 & $\begin{array}{l}\text { I have sufficient } \\
\text { ICT skills to } \\
\text { read messages } \\
\text { within } \\
<\text { CoP of the } \\
\text { organisation> }\end{array}$ & $\begin{array}{l}\text { Ik heb } \\
\text { voldoende ICT- } \\
\text { vaardigheden } \\
\text { om berichten } \\
\text { te lezen binnen } \\
\text { <CoP van de } \\
\text { organisatie> }\end{array}$ & Ability & $\begin{array}{l}\text { Deursen et al., } \\
(20 \mathrm{I})\end{array}$ \\
\hline A9 & $\begin{array}{l}\text { I have sufficient } \\
\text { ICT skills } \\
\text { to share } \\
\text { messages within } \\
<\text { CoP of the } \\
\text { organisation> }\end{array}$ & $\begin{array}{l}\text { Ik heb } \\
\text { voldoende ICT- } \\
\text { vaardigheden } \\
\text { om berichten } \\
\text { te delen binnen } \\
<\text { CoP van de } \\
\text { organisatie> }\end{array}$ & Digital skills & $\begin{array}{l}\text { Deursen et al., } \\
(20 \mathrm{II})\end{array}$ \\
\hline
\end{tabular}

\section{Adapted from or based on scales or}

Cadiz et al. (2009)

Cadiz et al.

$$
\text { (2009) }
$$

Deursen et al. (20II)
ICT skills to

$<$ CoP of the te lezen binnen $<$ CoP van de organisatie> vaardigheden $<$ CoP van de organisatie> 
Appendix 6.2: Loadings and cross-loadings.

\begin{tabular}{|c|c|c|c|c|c|c|c|c|c|}
\hline & 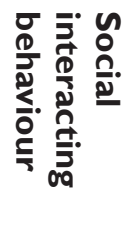 & 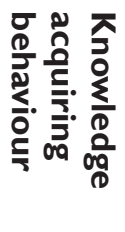 & $\begin{array}{l}\frac{5}{2} \\
\frac{0}{3} \\
\frac{3}{3} \\
\frac{2}{3}\end{array}$ & 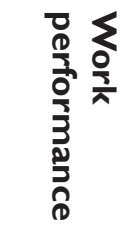 & 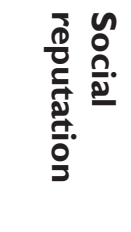 & 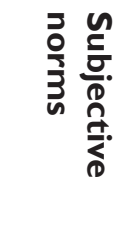 & $\frac{\text { Z }}{\overline{\bar{z}}}$ & 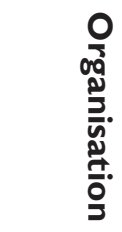 & $\begin{array}{l}\text { ด } \\
\text { D) } \\
\frac{0}{2} \\
\frac{1}{2}\end{array}$ \\
\hline S6 & 0.810 & 0.455 & 0.375 & $0.4 \mathrm{II}$ & 0.381 & -0.195 & 0.234 & 0.221 & 0.062 \\
\hline S7 & 0.843 & 0.487 & 0.403 & 0.444 & $0.34 I$ & -0.132 & 0.362 & 0.229 & 0.048 \\
\hline S8 & 0.853 & 0.591 & 0.513 & 0.487 & 0.437 & -0.100 & 0.333 & 0.265 & 0.075 \\
\hline S9 & 0.707 & 0.47 I & 0.422 & 0.421 & 0.415 & -0.108 & 0.232 & 0.165 & -0.042 \\
\hline SIO & 0.838 & 0.545 & 0.426 & 0.466 & 0.370 & -0.157 & 0.380 & 0.286 & 0.095 \\
\hline S5 & 0.580 & 0.922 & 0.574 & 0.478 & 0.337 & -0.207 & 0.399 & 0.482 & -0.023 \\
\hline SII & 0.448 & 0.702 & 0.366 & 0.369 & 0.225 & -0.165 & 0.200 & 0.112 & 0.223 \\
\hline MI & 0.324 & 0.387 & $0.74 I$ & 0.622 & 0.428 & -0.141 & 0.210 & 0.239 & 0.234 \\
\hline$M 2$ & 0.480 & 0.537 & 0.852 & 0.536 & 0.403 & -0.182 & 0.341 & 0.511 & 0.133 \\
\hline M3 & 0.435 & 0.531 & 0.868 & 0.577 & 0.403 & -0.166 & 0.242 & 0.419 & $0.25 I$ \\
\hline M4 & 0.417 & 0.468 & 0.828 & 0.653 & 0.467 & -0.167 & 0.331 & 0.347 & 0.133 \\
\hline M5 & 0.423 & 0.449 & 0.799 & 0.658 & 0.444 & -0.142 & 0.307 & 0.256 & 0.166 \\
\hline M6 & 0.464 & 0.476 & 0.744 & 0.643 & $0.57 \mid$ & -0.122 & 0.243 & $0 .|4|$ & 0.107 \\
\hline M7 & 0.490 & 0.474 & 0.670 & 0.788 & 0.573 & $-0.24 I$ & 0.291 & 0.215 & 0.163 \\
\hline M8 & 0.382 & 0.364 & 0.532 & 0.802 & 0.603 & -0.127 & $0.24 I$ & 0.053 & 0.130 \\
\hline M9 & 0.431 & 0.334 & 0.504 & 0.802 & 0.632 & -0.088 & 0.191 & -0.025 & 0.157 \\
\hline MIO & 0.457 & 0.406 & 0.614 & 0.904 & 0.679 & -0.198 & 0.164 & 0.126 & 0.224 \\
\hline MII & 0.429 & 0.435 & 0.655 & 0.852 & 0.703 & -0.229 & 0.180 & 0.155 & 0.291 \\
\hline MI2 & 0.494 & 0.447 & 0.649 & 0.880 & 0.676 & $-0.25 I$ & 0.214 & 0.166 & 0.279 \\
\hline MI3 & 0.481 & 0.430 & 0.576 & 0.892 & 0.651 & -0.237 & 0.247 & 0.146 & 0.262 \\
\hline
\end{tabular}




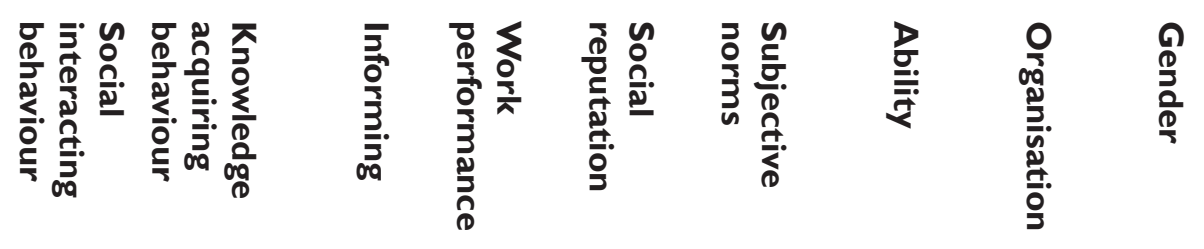

\begin{tabular}{llllllllll} 
MI5 & 0.465 & 0.500 & 0.758 & 0.807 & 0.572 & -0.159 & 0.287 & 0.202 & 0.156 \\
MI6 & 0.464 & 0.515 & 0.699 & 0.810 & 0.592 & -0.151 & 0.323 & 0.224 & 0.154 \\
MI7 & 0.516 & 0.445 & 0.647 & 0.859 & 0.603 & -0.207 & 0.252 & 0.266 & 0.203 \\
MI8 & 0.463 & 0.422 & 0.644 & 0.847 & 0.590 & -0.187 & 0.255 & 0.256 & 0.207 \\
MI4 & 0.480 & 0.353 & 0.560 & 0.742 & 1.000 & -0.119 & 0.169 & 0.077 & 0.127 \\
MI9 & -0.037 & -0.079 & -0.031 & -0.085 & 0.009 & 0.797 & 0.094 & -0.119 & -0.071 \\
M20 & -0.193 & -0.252 & -0.221 & -0.250 & -0.146 & 0.987 & -0.065 & -0.228 & -0.170 \\
AI & 0.361 & 0.347 & 0.255 & 0.225 & 0.096 & -0.039 & 0.849 & 0.267 & -0.109 \\
A2 & 0.373 & 0.371 & 0.267 & 0.221 & 0.090 & -0.029 & 0.837 & 0.267 & -0.088 \\
A3 & 0.147 & 0.207 & 0.114 & 0.060 & -0.029 & -0.073 & 0.614 & 0.200 & 0.066 \\
A4 & 0.248 & 0.315 & 0.299 & 0.185 & 0.049 & -0.122 & 0.722 & 0.217 & 0.032 \\
A5 & 0.297 & 0.218 & 0.217 & 0.246 & 0.104 & -0.002 & 0.773 & 0.236 & 0.029 \\
A6 & 0.328 & 0.252 & 0.255 & 0.303 & 0.257 & -0.024 & 0.711 & 0.193 & -0.005 \\
A7 & 0.261 & 0.342 & 0.355 & 0.234 & 0.153 & -0.010 & 0.786 & 0.229 & -0.059 \\
A8 & 0.303 & 0.316 & 0.324 & 0.259 & 0.213 & 0.023 & 0.849 & 0.224 & -0.107 \\
A9 & 0.295 & 0.304 & 0.299 & 0.238 & 0.205 & 0.020 & 0.804 & 0.179 & -0.099 \\
ORG & 0.290 & 0.417 & 0.403 & 0.200 & 0.077 & -0.217 & 0.289 & 1.000 & 0.052 \\
GEN & 0.061 & 0.075 & 0.208 & 0.242 & 0.127 & -0.158 & -0.060 & 0.052 & 1.000 \\
\hline
\end{tabular}




\section{Appendix 7.I: Items in the survey focused on the organisational perspective}

Items belonging to the levels of social behaviour

\begin{tabular}{l|l|l|l|l|l}
$\mathbf{1}$ & $\mathbf{2}$ & $\mathbf{3}$ & $\mathbf{4}$ & $\mathbf{5}$ & $\mathbf{6}$ \\
\hline Never & $\begin{array}{l}\text { Less frequent } \\
\text { than once a } \\
\text { week }\end{array}$ & Once a week & $\begin{array}{l}\text { Multiple } \\
\text { times a week }\end{array}$ & Once a day & $\begin{array}{l}\text { Multiple } \\
\text { times a day }\end{array}$
\end{tabular}

$\begin{array}{ll}\text { \# English translation } & \begin{array}{l}\text { Original items in } \\ \text { of the items: }\end{array}\end{array}$

Adapted from or based on scales or findings from:

P5 I read, watch or listen Ik lees, bekijk to the information that of beluister de others share

P7 I share information that is / can be relevant for colleagues within my own organisation. This may include: (work) reports and / or official documents, methodology I methods, (practical) knowledge and / or (work) experiences

P8 I appreciate the contributions of colleagues. This can be with a like, thanks, etc. informatie die anderen delen

Ik deel informatie dat voor collega's binnen mijn eigen organisatie relevant is/kan zijn.

Dit kan zijn: (werk) rapporten en/of officiële documenten, methodiek/methoden, (praktische) kennis en/of (werk) ervaringen

Ik waardeer de bijdragen van collega's. Dit kan zijn: met een like, dank, etc.

P9 I get to know (new) colleagues within my organisation
Ik leer (nieuwe) collega's binnen mijn organisatie kennen
Knowledge acquisition behaviour

Social interaction behaviour

Bock et al. (2005); Jeon et al. (20I I); van den Hooff \& de Leeuw van Weenen (2004)
Millen et al., (2002)
Social interaction behaviour Social interaction behaviour
Bock et al. (2005)

Chiu et al. (2006); Ruuska, I., \& Vartiainen (2005) 


\begin{tabular}{|c|c|c|c|c|}
\hline \# & $\begin{array}{l}\text { English translation } \\
\text { of the items: }\end{array}$ & $\begin{array}{l}\text { Original items in } \\
\text { Dutch: }\end{array}$ & Concept: & $\begin{array}{l}\text { Adapted from } \\
\text { or based on } \\
\text { scales or } \\
\text { findings from: }\end{array}$ \\
\hline PIO & $\begin{array}{l}\text { I communicate with } \\
\text { colleagues within my } \\
\text { organisation. This can } \\
\text { be participation in } \\
\text { discussions, making } \\
\text { appointments, etc. }\end{array}$ & $\begin{array}{l}\text { Ik communiceer met } \\
\text { collega's binnen mijn } \\
\text { organisatie. Dit kan } \\
\text { zijn: deelname in } \\
\text { discussies, maken van } \\
\text { afspraken, etc. }\end{array}$ & $\begin{array}{l}\text { Social } \\
\text { interaction } \\
\text { behaviour }\end{array}$ & Context-based \\
\hline P6 & $\begin{array}{l}\text { I ask colleagues within } \\
\text { my organisation for } \\
\text { information relevant } \\
\text { to me. This can be: } \\
\text { (work) reports, } \\
\text { official documents, } \\
\text { methodology / } \\
\text { methods, (practical) } \\
\text { knowledge and } \\
\text { experiences }\end{array}$ & $\begin{array}{l}\text { Ik vraag collega's } \\
\text { binnen mijn } \\
\text { organisatie naar } \\
\text { voor mij relevante } \\
\text { informatie. Dit kan } \\
\text { zijn: (werk)rapporten, } \\
\text { officiële documenten, } \\
\text { methodiek/methoden, } \\
\text { (praktische) kennis } \\
\text { en ervaringen }\end{array}$ & $\begin{array}{l}\text { Social } \\
\text { interaction } \\
\text { behaviour }\end{array}$ & $\begin{array}{l}\text { van den Hooff } \\
\text { \& de Leeuw van } \\
\text { Weenen }(2004)\end{array}$ \\
\hline PII & $\begin{array}{l}\text { I read the newsletter } \\
\text { with recent activities, } \\
\text { which I receive } \\
\text { periodically }\end{array}$ & $\begin{array}{l}\text { Ik lees de } \\
\text { samenvattende } \\
\text { mail met recente } \\
\text { activiteiten, die ik } \\
\text { periodiek ontvang }\end{array}$ & $\begin{array}{l}\text { Knowledge } \\
\text { acquisition } \\
\text { behaviour }\end{array}$ & Context-based \\
\hline
\end{tabular}

Items belonging to the Opportunity

\begin{tabular}{l|l|l|l|l|l|l}
$\mathbf{I}$ & $\mathbf{2}$ & $\mathbf{3}$ & $\mathbf{4}$ & $\mathbf{5}$ & $\mathbf{6}$ & $\mathbf{7}$ \\
\hline $\begin{array}{l}\text { Strongly } \\
\text { disagree }\end{array}$ & Disagree & $\begin{array}{l}\text { More } \\
\text { or less } \\
\text { disagree }\end{array}$ & Undecided & $\begin{array}{l}\text { More or } \\
\text { less agree }\end{array}$ & Agree & $\begin{array}{l}\text { Strongly } \\
\text { agree }\end{array}$
\end{tabular}

\begin{tabular}{llll}
\hline English translation & $\begin{array}{l}\text { Original items in } \\
\text { of the items: }\end{array}$ & Concept: & $\begin{array}{l}\text { Adapted from } \\
\text { or based on } \\
\text { scales or }\end{array}$ \\
& & & $\begin{array}{l}\text { findings from: } \\
\text { fing }\end{array}$ \\
\hline
\end{tabular}

OI During a working day I have little time to read contributions from others
Gedurende een werkdag heb ik weinig tijd om bijdragen van anderen te lezen
Time

Orlikowski 


\begin{tabular}{|c|c|c|c|c|}
\hline \# & $\begin{array}{l}\text { English translation } \\
\text { of the items: }\end{array}$ & $\begin{array}{l}\text { Original items in } \\
\text { Dutch: }\end{array}$ & Concept: & $\begin{array}{l}\text { Adapted from } \\
\text { or based on } \\
\text { scales or } \\
\text { findings from: }\end{array}$ \\
\hline $\mathrm{O} 2$ & $\begin{array}{l}\text { During a working day } \\
\text { I have little time to } \\
\text { contribute myself }\end{array}$ & $\begin{array}{l}\text { Gedurende een } \\
\text { werkdag heb ik } \\
\text { weinig tijd om zelf } \\
\text { een bijdrage te } \\
\text { leveren }\end{array}$ & Time & $\begin{array}{l}\text { Orlikowski } \\
\text { (1993) }\end{array}$ \\
\hline O3 & $\begin{array}{l}\text { I have enough } \\
\text { autonomy to } \\
\text { determine whether I } \\
\text { want to participate }\end{array}$ & $\begin{array}{l}\text { Ik heb voldoende } \\
\text { autonomie om te } \\
\text { bepalen of ik wil } \\
\text { deelnemen }\end{array}$ & Work autonomy & $\begin{array}{l}\text { Salanova, Peiró \& } \\
\text { Schaufeli, (2002); } \\
\text { Cardona et al., } \\
(2003)\end{array}$ \\
\hline O4 & $\begin{array}{l}\text { I have enough } \\
\text { autonomy to } \\
\text { determine when I want } \\
\text { to participate }\end{array}$ & $\begin{array}{l}\text { Ik heb voldoende } \\
\text { autonomie om te } \\
\text { bepalen wanneer ik } \\
\text { wil participeren }\end{array}$ & Work autonomy & $\begin{array}{l}\text { Salanova, Peiró \& } \\
\text { Schaufeli, (2002); } \\
\text { Cardona et al., } \\
\text { (2003) }\end{array}$ \\
\hline O5 & $\begin{array}{l}\text { I have enough } \\
\text { autonomy to } \\
\text { determine what I want } \\
\text { to add }\end{array}$ & $\begin{array}{l}\text { Ik heb voldoende } \\
\text { autonomie om te } \\
\text { bepalen wat ik wil } \\
\text { toevoegen }\end{array}$ & Work autonomy & $\begin{array}{l}\text { Salanova, Peiró \& } \\
\text { Schaufeli, (2002); } \\
\text { Cardona et al., } \\
\text { (2003) }\end{array}$ \\
\hline O6 & $\begin{array}{l}\text { I receive enough } \\
\text { technical support } \\
\text { to use the digital } \\
\text { technologies facilitating } \\
\text { the <CoP of the } \\
\text { organisation>. E.g. } \\
\text { through training and } \\
\text { education. }\end{array}$ & $\begin{array}{l}\text { Ik krijg voldoende } \\
\text { technische } \\
\text { ondersteuning om } \\
\text { de }<\text { CoP van de } \\
\text { organisatie> te } \\
\text { kunnen gebruiken. } \\
\text { Bijv. door trainingen } \\
\text { en opleidingen. }\end{array}$ & $\begin{array}{l}\text { Facilitating } \\
\text { conditions }\end{array}$ & $\begin{array}{l}\text { Bhattacherjee, } \\
(200 \mathrm{I}) \text {; } \\
\text { Venkatesh, } \\
(2000)\end{array}$ \\
\hline O7 & $\begin{array}{l}\text { I receive enough } \\
\text { support in the way } \\
\text { we can use the digital } \\
\text { technologies facilitating } \\
\text { the <CoP of the } \\
\text { organisation>. E.g. } \\
\text { through training and } \\
\text { education. }\end{array}$ & $\begin{array}{l}\text { Ik krijg voldoende } \\
\text { ondersteuning in } \\
\text { de wijze waarop } \\
\text { we <CoP van de } \\
\text { organisatie> kunnen } \\
\text { gebruiken. Bijv. } \\
\text { door trainingen en } \\
\text { opleidingen. }\end{array}$ & $\begin{array}{l}\text { Facilitating } \\
\text { conditions }\end{array}$ & $\begin{array}{l}\text { Bhattacherjee, } \\
(200 \mathrm{I}) ; \\
\text { Venkatesh, } \\
(2000)\end{array}$ \\
\hline
\end{tabular}


Appendix 7.2: Loadings and cross-loadings.

\begin{tabular}{|c|c|c|c|c|c|c|c|}
\hline & $\begin{array}{l}\text { Social } \\
\text { Interac- } \\
\text { tion Be- } \\
\text { haviour }\end{array}$ & $\begin{array}{l}\text { Knowl- } \\
\text { edge } \\
\text { Acquisi- } \\
\text { tion Be- } \\
\text { haviour }\end{array}$ & Time & $\begin{array}{l}\text { Work } \\
\text { autono- } \\
\text { my }\end{array}$ & $\begin{array}{l}\text { Facilitat- } \\
\text { ing con- } \\
\text { ditions }\end{array}$ & $\begin{array}{l}\text { Organi- } \\
\text { sation }\end{array}$ & Gender \\
\hline S6 & 0.811 & 0.448 & 0.072 & 0.217 & 0.272 & 0.221 & 0.062 \\
\hline S7 & 0.859 & 0.485 & 0.105 & 0.258 & 0.401 & 0.229 & 0.048 \\
\hline S8 & 0.849 & 0.593 & 0.075 & 0.229 & 0.372 & 0.265 & 0.075 \\
\hline S9 & 0.665 & 0.468 & 0.115 & 0.181 & $0.24 I$ & 0.165 & -0.042 \\
\hline SIO & 0.855 & 0.538 & 0.051 & 0.254 & 0.408 & 0.286 & 0.095 \\
\hline S5 & 0.577 & 0.956 & 0.164 & 0.364 & 0.473 & 0.482 & -0.023 \\
\hline SII & 0.447 & 0.626 & 0.083 & 0.104 & 0.209 & 0.112 & 0.223 \\
\hline OI & 0.121 & 0.169 & 0.981 & 0.134 & 0.234 & 0.134 & 0.015 \\
\hline $\mathrm{O} 2$ & 0.063 & 0.146 & 0.966 & 0.156 & 0.212 & 0.127 & 0.034 \\
\hline $\mathrm{O} 3$ & 0.240 & 0.266 & 0.181 & 0.836 & 0.400 & 0.105 & -0.120 \\
\hline O4 & 0.282 & 0.313 & 0.144 & 0.904 & 0.421 & 0.203 & -0.041 \\
\hline O5 & 0.223 & 0.310 & 0.065 & 0.894 & 0.451 & 0.238 & -0.107 \\
\hline O6 & 0.416 & 0.436 & 0.234 & 0.482 & 0.980 & 0.309 & -0.049 \\
\hline O7 & 0.425 & 0.47 I & 0.219 & 0.465 & 0.982 & 0.348 & -0.071 \\
\hline ORG & 0.293 & 0.440 & 0.134 & 0.210 & 0.335 & 1.000 & 0.052 \\
\hline GEN & 0.068 & 0.051 & 0.024 & -0.099 & -0.062 & 0.052 & 1.000 \\
\hline
\end{tabular}




\section{Appendix 8.I: Items in the survey focused on the individual variables}

Items belonging to the levels of social behaviour

$\begin{array}{llllll}\mathbf{1} & \mathbf{2} & \mathbf{3} & \mathbf{4} & \mathbf{5} & \mathbf{6} \\ \text { Never } & \begin{array}{l}\text { Less frequent } \\ \text { than once a } \\ \text { week }\end{array} & \text { Once a week } & \begin{array}{l}\text { Multiple } \\ \text { times a week }\end{array} & \text { Once a day } & \begin{array}{l}\text { Multiple } \\ \text { times a day }\end{array}\end{array}$

$\begin{array}{ll}\text { English } & \text { Original items Concept: } \\ \text { translation of in Dutch: } \\ \text { the items: }\end{array}$

P5

\begin{tabular}{|c|c|c|c|c|}
\hline P5 & $\begin{array}{l}\text { I read, watch } \\
\text { or listen to the } \\
\text { information that } \\
\text { others share }\end{array}$ & $\begin{array}{l}\text { Ik lees, bekijk } \\
\text { of beluister de } \\
\text { informatie die } \\
\text { anderen delen }\end{array}$ & $\begin{array}{l}\text { Knowledge } \\
\text { acquisition } \\
\text { behaviour }\end{array}$ & $\begin{array}{l}\text { Millen et al., } \\
(2002)\end{array}$ \\
\hline P7 & $\begin{array}{l}\text { I share } \\
\text { information } \\
\text { that is / can } \\
\text { be relevant } \\
\text { for colleagues } \\
\text { within my own } \\
\text { organisation. } \\
\text { This may include: } \\
\text { (work) reports } \\
\text { and / or official } \\
\text { documents, } \\
\text { methodology } \\
\text { / methods, } \\
\text { (practical) } \\
\text { knowledge and } \\
\text { / or (work) } \\
\text { experiences }\end{array}$ & $\begin{array}{l}\text { Ik deel } \\
\text { informatie dat } \\
\text { voor collega's } \\
\text { binnen mijn } \\
\text { eigen organisatie } \\
\text { relevant is/kan } \\
\text { zijn. Dit kan zijn: } \\
\text { (werk)rapporten } \\
\text { en/of officiële } \\
\text { documenten, } \\
\text { methodiek/ } \\
\text { methoden, } \\
\text { (praktische) } \\
\text { kennis en/ } \\
\text { of (werk) } \\
\text { ervaringen }\end{array}$ & $\begin{array}{l}\text { Social } \\
\text { interaction } \\
\text { behaviour }\end{array}$ & $\begin{array}{l}\text { Bock et al. } \\
\text { (2005); Jeon et } \\
\text { al. (20II); van } \\
\text { den Hooff \& } \\
\text { de Leeuw van } \\
\text { Weenen (2004) }\end{array}$ \\
\hline P8 & $\begin{array}{l}\text { I appreciate the } \\
\text { contributions of } \\
\text { colleagues. This } \\
\text { can be with a } \\
\text { like, thanks, etc. }\end{array}$ & $\begin{array}{l}\text { Ik waardeer de } \\
\text { bijdragen van } \\
\text { collega's. Dit kan } \\
\text { zijn: met een } \\
\text { like, dank, etc. }\end{array}$ & $\begin{array}{l}\text { Social } \\
\text { interaction } \\
\text { behaviour }\end{array}$ & $\begin{array}{l}\text { Bock et al. } \\
(2005)\end{array}$ \\
\hline
\end{tabular}

\section{Adapted from or based on scales or findings from:}




\begin{tabular}{|c|c|c|c|c|}
\hline \# & $\begin{array}{l}\text { English } \\
\text { translation of } \\
\text { the items: }\end{array}$ & $\begin{array}{l}\text { Original items } \\
\text { in Dutch: }\end{array}$ & Concept: & $\begin{array}{l}\text { Adapted from } \\
\text { or based on } \\
\text { scales or } \\
\text { findings from: }\end{array}$ \\
\hline P9 & $\begin{array}{l}\text { I get to know } \\
\text { (new) colleagues } \\
\text { within my } \\
\text { organisation }\end{array}$ & $\begin{array}{l}\text { Ik leer (nieuwe) } \\
\text { collega's binnen } \\
\text { mijn organisatie } \\
\text { kennen }\end{array}$ & $\begin{array}{l}\text { Social } \\
\text { interaction } \\
\text { behaviour }\end{array}$ & $\begin{array}{l}\text { Chiu et al. } \\
\text { (2006); Ruuska, } \\
\text { l., \& Vartiainen } \\
(2005)\end{array}$ \\
\hline PIO & $\begin{array}{l}\text { I communicate } \\
\text { with colleagues } \\
\text { within my } \\
\text { organisation. } \\
\text { This can be } \\
\text { participation } \\
\text { in discussions, } \\
\text { making } \\
\text { appointments, } \\
\text { etc. }\end{array}$ & $\begin{array}{l}\text { Ik communiceer } \\
\text { met collega's } \\
\text { binnen mijn } \\
\text { organisatie. } \\
\text { Dit kan zijn: } \\
\text { deelname in } \\
\text { discussies, } \\
\text { maken van } \\
\text { afspraken, etc. }\end{array}$ & $\begin{array}{l}\text { Social } \\
\text { interaction } \\
\text { behaviour }\end{array}$ & Context-based \\
\hline P6 & $\begin{array}{l}\text { I ask colleagues } \\
\text { within my } \\
\text { organisation } \\
\text { for information } \\
\text { relevant to } \\
\text { me. This can } \\
\text { be: (work) } \\
\text { reports, official } \\
\text { documents, } \\
\text { methodology } \\
\text { / methods, } \\
\text { (practical) } \\
\text { knowledge and } \\
\text { experiences }\end{array}$ & $\begin{array}{l}\text { Ik vraag collega's } \\
\text { binnen mijn } \\
\text { organisatie } \\
\text { naar voor } \\
\text { mij relevante } \\
\text { informatie. Dit } \\
\text { kan zijn: (werk) } \\
\text { rapporten, } \\
\text { officiële } \\
\text { documenten, } \\
\text { methodiek/ } \\
\text { methoden, } \\
\text { (praktische) } \\
\text { kennis en } \\
\text { ervaringen }\end{array}$ & $\begin{array}{l}\text { Social } \\
\text { interaction } \\
\text { behaviour }\end{array}$ & $\begin{array}{l}\text { van den Hooff } \\
\text { \& de Leeuw van } \\
\text { Weenen (2004) }\end{array}$ \\
\hline PII & $\begin{array}{l}\text { I read the } \\
\text { newsletter with } \\
\text { recent activities, } \\
\text { which I receive } \\
\text { periodically }\end{array}$ & $\begin{array}{l}\text { Ik lees de } \\
\text { samenvattende } \\
\text { mail met recente } \\
\text { activiteiten, die } \\
\text { ik periodiek } \\
\text { ontvang }\end{array}$ & $\begin{array}{l}\text { Knowledge } \\
\text { acquisition } \\
\text { behaviour }\end{array}$ & Context-based \\
\hline
\end{tabular}


Items belonging to the Communality

$\begin{array}{lllllll}\text { I } & \mathbf{2} & \mathbf{3} & \mathbf{4} & \mathbf{5} & \mathbf{6} & \mathbf{7} \\ \begin{array}{l}\text { Strongly } \\ \text { disagree }\end{array} & \text { Disagree } & \begin{array}{l}\text { More } \\ \text { or less } \\ \text { disagree }\end{array} & \text { Undecided } & \begin{array}{l}\text { More or } \\ \text { less agree }\end{array} & \text { Agree } & \begin{array}{l}\text { Strongly } \\ \text { agree }\end{array}\end{array}$

$\begin{array}{ll}\text { \# English translation of } & \begin{array}{l}\text { Original items in } \\ \text { the items: }\end{array} \\ \text { Dutch: }\end{array}$

\section{Concept: Adapted from or based on scales or findings from:}

$\mathrm{Cl} \quad$ In the group (s) in which I participate, we use common jargon

In de groep(en) waarin

Shared

Chiu et al. ik deelneem hanteren we gemeenschappelijk language (2006) jargon

C2 The members - within

De leden - binnen de Shared Chiu et al. the group (s) in which I groep(en) waarin ik participate - discuss in an deelneem - discussiëren understandable way op een begrijpelijke manier

C3 The members - within

De leden - binnen de

Shared Chiu et al. the group (s) in which I participate - use groep(en) waarin ik deelneem - gebruiken language (2006) understandable language in their messages or articles begrijpelijke taal in hun berichten of artikelen

C4 There is no hierarchical

$\mathrm{Er}$ is geen hiërarchische

Shared ranking within the group rangorde binnen de leadership

Wood (2005) (s) in which I participate groep(en) waarin ik deelneem

C5 There is equality within

Er is gelijkheid binnen

Shared Wood (2005) the group (s) in which de groep(en) waarin ik deelneem, ongeacht I participate, regardless de 'titels' die anderen of the 'titles' that binnen de organisatie others carry within the organisation dragen leadership (2006) 


\begin{tabular}{lll}
\hline English translation of & $\begin{array}{l}\text { Original items in } \\
\text { Dutch: }\end{array}$ & $\begin{array}{c}\text { Concept: } \\
\text { the items: }\end{array}$ \\
& & from or \\
& & based on \\
& & scales or \\
& & findings from: \\
& & \\
\hline
\end{tabular}
$\begin{array}{llll}\text { C6 There is not one person } & \begin{array}{l}\text { Er is niet één persoon } \\ \text { who makes all decisions }\end{array} & \begin{array}{l}\text { Shared alle beslissingen } \\ \text { leadership }\end{array} & \text { Wood (2005) }\end{array}$ within the group (s) in neemt binnen de which I participate groep(en) waarin ik deelneem

C7 There is not a feeling of Er heerst niet een gevoel Shared Wood (2005) "every man for himself" van "ieder voor zich" leadership within the group (s) in binnen de groep(en) which I participate waarin ik deelneem

C8 In the group (s) in which In de groep(en) waarin I participate, we share ik deelneem delen we common norms and gezamenlijke normen en Shared Chiu et al. identity (2006) values waarden

C9 In the group (s) in which In de groep(en) waarin I participate I feel a sense ik deelneem heb ik het of belonging gevoel dat ik erbij hoor

Shared Chiu et al. identity (2006) 
Appendix 8.2: Loadings and cross-loadings.

\begin{tabular}{|c|c|c|c|c|c|c|c|}
\hline & 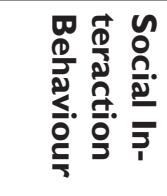 & 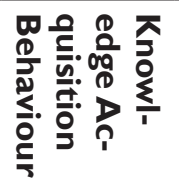 & 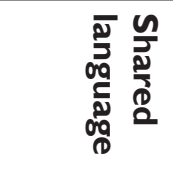 & 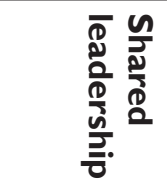 & 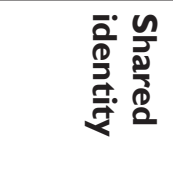 & 응. 옴 & \\
\hline S6 & 0.809 & 0.448 & 0.290 & 0.305 & 0.246 & 0.221 & 0.062 \\
\hline S7 & 0.855 & 0.485 & 0.345 & 0.374 & 0.379 & 0.229 & 0.048 \\
\hline S8 & 0.849 & 0.593 & 0.321 & 0.363 & 0.396 & 0.265 & 0.075 \\
\hline S9 & 0.661 & 0.468 & 0.241 & 0.194 & 0.225 & 0.165 & -0.042 \\
\hline SIO & 0.863 & 0.537 & 0.448 & 0.417 & 0.470 & 0.286 & 0.095 \\
\hline S5 & 0.577 & 0.958 & 0.386 & 0.416 & 0.396 & 0.482 & -0.023 \\
\hline SII & 0.448 & 0.621 & 0.229 & 0.153 & 0.231 & 0.112 & 0.223 \\
\hline $\mathrm{Cl}$ & 0.401 & 0.333 & 0.886 & 0.550 & 0.598 & $0.28 \mathrm{I}$ & 0.125 \\
\hline $\mathrm{C} 2$ & 0.342 & 0.359 & 0.916 & 0.625 & 0.620 & 0.224 & 0.075 \\
\hline $\mathrm{C} 3$ & 0.391 & 0.386 & 0.919 & 0.661 & 0.624 & 0.257 & 0.124 \\
\hline C4 & 0.428 & 0.406 & 0.662 & 0.971 & 0.642 & 0.251 & 0.128 \\
\hline C5 & 0.388 & 0.363 & 0.645 & 0.964 & 0.648 & 0.233 & 0.085 \\
\hline C8 & 0.360 & 0.329 & 0.584 & 0.583 & 0.879 & 0.184 & 0.005 \\
\hline $\mathrm{C} 9$ & 0.430 & 0.396 & 0.630 & 0.613 & 0.917 & 0.298 & 0.081 \\
\hline ORG & 0.294 & $0.44 I$ & $0.28 I$ & 0.251 & 0.274 & 1.000 & 0.052 \\
\hline GEN & 0.069 & 0.049 & 0.120 & 0.111 & 0.052 & 0.052 & 1.000 \\
\hline
\end{tabular}


Appendix 10.1: Questionnaire that is connected to the web-based social interaction data

\begin{tabular}{l|l|l|l|l|l|l}
1 & 2 & 3 & 4 & 5 & 6 & 7 \\
\hline $\begin{array}{l}\text { Strongly } \\
\text { agree }\end{array}$ & Agree & $\begin{array}{l}\text { More or } \\
\text { less agree }\end{array}$ & Undecided & $\begin{array}{l}\text { More } \\
\text { or less } \\
\text { disagree }\end{array}$ & Disagree & $\begin{array}{l}\text { Strongly } \\
\text { disagree }\end{array}$
\end{tabular}

Items belonging to the Motivation

\begin{tabular}{llllll}
\hline$\#$ & $\begin{array}{l}\text { English trans- } \\
\text { lation of the } \\
\text { items: }\end{array}$ & $\begin{array}{l}\text { Original items in } \\
\text { Dutch: }\end{array}$ & Concept: & $\begin{array}{l}\text { Adapted from } \\
\text { or based on } \\
\text { scales or find- } \\
\text { ings from: }\end{array}$ & $\begin{array}{l}\text { In- } \\
\text { cluded } \\
\text { in } \\
\text { scale }^{5}\end{array}$ \\
\hline MI & $\begin{array}{l}\text { To be informed } \\
\text { of the latest } \\
\text { developments } \\
\text { within my area of } \\
\text { expertise }\end{array}$ & $\begin{array}{l}\text { Om op de hoogte } \\
\text { te zijn van de laatste } \\
\text { ontwikkelingen bin- }\end{array}$ & Informing & Lee \& Ma (20I2) & No \\
& & & & \\
& & & & \\
\end{tabular}

M2 To be informed of the latest

Om op de hoogte Informing Lee \& $M a(2012)$ No developments te zijn van de laatste within the ontwikkelingen

organisation binnen de organisatie

M3 To know which activities are planned

Om te weten welke Informing Lee \& Ma (20I2) No activiteiten er op de planning staan

M4 To search for information that

Om naar informati Informing Lee \& Ma (20I2) Yes is relevant to my work te zoeken die relevant is voor het uitvoeren van mijn werk

M5 To find

Om informatie op Informing Lee \& Ma (2012) Yes information later een later moment terug te vinden

M6 To see what others do

Om te zien wat Informing Leonardi (2014) Yes anderen doen 


\begin{tabular}{llllll}
\hline$\#$ & $\begin{array}{l}\text { English trans- } \\
\text { lation of the } \\
\text { items: }\end{array}$ & $\begin{array}{l}\text { Original items in } \\
\text { Dutch: }\end{array}$ & Concept: & $\begin{array}{l}\text { Adapted from } \\
\text { or based on } \\
\text { scales or find- } \\
\text { ings from: }\end{array}$ & $\begin{array}{l}\text { In- } \\
\text { cluded } \\
\text { in } \\
\text { scale }^{5}\end{array}$ \\
\hline M7 & $\begin{array}{l}\text { To share } \\
\text { knowledge, } \\
\text { insights and } \\
\text { experiences }\end{array}$ & $\begin{array}{l}\text { Om ervaringen, } \\
\text { kennis en inzichten } \\
\text { te delen }\end{array}$ & $\begin{array}{l}\text { Work } \\
\text { performance }\end{array}$ & $\begin{array}{l}\text { Kankanhalli et al. } \\
(2005)\end{array}$ & Yes \\
\end{tabular}

M8 To contribute in subject-related discussions

Om bij te dragen in vakinhoudelijke

Work Xu, Quaddus, \& Yes performance Gao (2014)

M9 To contribute Om online bij te

Work

Context-driven Yes online in projects dragen in projecten performance

(Xu, Quaddus, \& Gao, 20I4)

MIO To get support

Om ondersteuning from other van andere professionals professionals te krijgen

Work Han et al. (2007) Yes performance

Lee \& Ma (20I2);

Bock et al. (2005)

MII To get in touch with other professionals

Om in contact te

Work

Lee \& Ma (20I2); Yes komen met andere professionals performance

Bock et al. (2005);

Deursen et al. (20II)

MI2 To stay in Om in contact te Work Lee \& Ma (20I2); Yes contact with other professionals blijven met andere professionals performance Bock et al. (2005); Deursen et al. (20II)

MI3 To share ideas

Om ideeën te

Work

Lee \& Ma (20I2); Yes with other delen met andere professionals professionals

MI4 To present myself

MI5 To continuously improve my work practices

MI6 Because it clearly has added value to perform my job
Om mij te profileren

Om mijn werk steeds beter te kunnen doen

Omdat het duidelijk meerwaarde heeft voor het uitvoeren van mijn werk
Social reputation

Work Xu, Quaddus, \& Yes performance Gao (20I4)

Work Context-based Yes performance
Lee \& Ma (20I2) Yes 


\begin{tabular}{|c|c|c|c|c|c|}
\hline$\#$ & $\begin{array}{l}\text { English trans- } \\
\text { lation of the } \\
\text { items: }\end{array}$ & $\begin{array}{l}\text { Original items in } \\
\text { Dutch: }\end{array}$ & Concept: & $\begin{array}{l}\text { Adapted from } \\
\text { or based on } \\
\text { scales or find- } \\
\text { ings from: }\end{array}$ & $\begin{array}{l}\text { In- } \\
\text { cluded } \\
\text { in } \\
\text { scale }^{5}\end{array}$ \\
\hline MI7 & $\begin{array}{l}\text { To perform (part } \\
\text { of) my work } \\
\text { independently of } \\
\text { place }\end{array}$ & $\begin{array}{l}\text { Om (een deel } \\
\text { van) mijn werk } \\
\text { onafhankelijk van } \\
\text { plaats uit te voeren }\end{array}$ & $\begin{array}{l}\text { Work } \\
\text { performance }\end{array}$ & $\begin{array}{l}\text { Kulkarni et al. } \\
\text { (2007) }\end{array}$ & Yes \\
\hline MI8 & $\begin{array}{l}\text { To perform (part } \\
\text { of) my work } \\
\text { independently of } \\
\text { time }\end{array}$ & $\begin{array}{l}\text { Om (een deel } \\
\text { van) mijn werk } \\
\text { onafhankelijk van tijd } \\
\text { uit te voeren }\end{array}$ & $\begin{array}{l}\text { Work } \\
\text { performance }\end{array}$ & $\begin{array}{l}\text { Kulkarni et al. } \\
\text { (2007) }\end{array}$ & Yes \\
\hline MI9 & $\begin{array}{l}\text { Because I feel } \\
\text { pressure from } \\
\text { the organisation } \\
\text { to participate }\end{array}$ & $\begin{array}{l}\text { Omdat ik vanuit de } \\
\text { organisatie druk voel } \\
\text { om deel te nemen }\end{array}$ & $\begin{array}{l}\text { Subjective } \\
\text { norm }\end{array}$ & $\begin{array}{l}\text { Hung, Lai, \& } \\
\text { Chou, (20I5); } \\
\text { Scarbrough } \\
\text { (2003) }\end{array}$ & No \\
\hline M20 & $\begin{array}{l}\text { Because I feel } \\
\text { pressure from } \\
\text { my colleagues to } \\
\text { participate }\end{array}$ & $\begin{array}{l}\text { Omdat ik vanuit mijn } \\
\text { collega's druk voel } \\
\text { om deel te nemen }\end{array}$ & $\begin{array}{l}\text { Subjective } \\
\text { norm }\end{array}$ & $\begin{array}{l}\text { Hung et al. } \\
(2015) ; \text { Lewis et } \\
\text { al., (2003) }\end{array}$ & No \\
\hline
\end{tabular}

Items belonging to the opportunity

\begin{tabular}{|c|c|c|c|c|c|}
\hline \# & $\begin{array}{l}\text { English trans- } \\
\text { lation of the } \\
\text { items: }\end{array}$ & $\begin{array}{l}\text { Original items in } \\
\text { Dutch: }\end{array}$ & Concept: & $\begin{array}{l}\text { Adapted from } \\
\text { or based on } \\
\text { scales or find- } \\
\text { ings from: }\end{array}$ & $\begin{array}{l}\text { Includ } \\
\text { ed in } \\
\text { scale }\end{array}$ \\
\hline Ol & $\begin{array}{l}\text { During a work- } \\
\text { ing day I have lit- } \\
\text { tle time to read } \\
\text { contributions } \\
\text { from others }\end{array}$ & $\begin{array}{l}\text { Gedurende een } \\
\text { werkdag heb ik } \\
\text { weinig tijd om bi- } \\
\text { jdragen van anderen } \\
\text { te lezen }\end{array}$ & Time & $\begin{array}{l}\text { Orlikowski } \\
\text { (1993) }\end{array}$ & No \\
\hline $\mathrm{O} 2$ & $\begin{array}{l}\text { During a } \\
\text { working day I } \\
\text { have little time } \\
\text { to contribute } \\
\text { myself }\end{array}$ & $\begin{array}{l}\text { Gedurende een } \\
\text { werkdag heb ik } \\
\text { weinig tijd om zelf } \\
\text { een bijdrage te } \\
\text { leveren }\end{array}$ & Time & $\begin{array}{l}\text { Orlikowski } \\
\text { (I993) }\end{array}$ & No \\
\hline
\end{tabular}




\begin{tabular}{|c|c|c|c|c|c|}
\hline \# & $\begin{array}{l}\text { English trans- } \\
\text { lation of the } \\
\text { items: }\end{array}$ & $\begin{array}{l}\text { Original items in } \\
\text { Dutch: }\end{array}$ & Concept: & $\begin{array}{l}\text { Adapted from } \\
\text { or based on } \\
\text { scales or find- } \\
\text { ings from: }\end{array}$ & $\begin{array}{l}\text { Includ- } \\
\text { ed in } \\
\text { scale }\end{array}$ \\
\hline O3 & $\begin{array}{l}\text { I have enough } \\
\text { autonomy to } \\
\text { determine } \\
\text { whether I want } \\
\text { to participate }\end{array}$ & $\begin{array}{l}\text { Ik heb voldoende } \\
\text { autonomie om te } \\
\text { bepalen of ik wil } \\
\text { deelnemen }\end{array}$ & $\begin{array}{l}\text { Work au- } \\
\text { tonomy }\end{array}$ & $\begin{array}{l}\text { Salanova, Peiró \& } \\
\text { Schaufeli, (2002); } \\
\text { Cardona et al., } \\
(2003)\end{array}$ & Yes \\
\hline O4 & $\begin{array}{l}\text { I have enough } \\
\text { autonomy to } \\
\text { determine when } \\
\text { I want to partic- } \\
\text { ipate }\end{array}$ & $\begin{array}{l}\text { Ik heb voldoende } \\
\text { autonomie om te } \\
\text { bepalen wanneer ik } \\
\text { wil participeren }\end{array}$ & $\begin{array}{l}\text { Work au- } \\
\text { tonomy }\end{array}$ & $\begin{array}{l}\text { Salanova, Peiró \& } \\
\text { Schaufeli, (2002); } \\
\text { Cardona et al., } \\
(2003)\end{array}$ & Yes \\
\hline O5 & $\begin{array}{l}\text { I have enough } \\
\text { autonomy to } \\
\text { determine what I } \\
\text { want to add }\end{array}$ & $\begin{array}{l}\text { Ik heb voldoende } \\
\text { autonomie om te } \\
\text { bepalen wat ik wil } \\
\text { toevoegen }\end{array}$ & $\begin{array}{l}\text { Work au- } \\
\text { tonomy }\end{array}$ & $\begin{array}{l}\text { Salanova, Peiró \& } \\
\text { Schaufeli, (2002); } \\
\text { Cardona et al., } \\
(2003)\end{array}$ & Yes \\
\hline 06 & $\begin{array}{l}\text { I receive enough } \\
\text { technical sup- } \\
\text { port to use the } \\
\text { digital technol- } \\
\text { ogies facilitating } \\
\text { the <CoP of the } \\
\text { Organisation>. } \\
\text { E.g. through } \\
\text { training and } \\
\text { education. }\end{array}$ & $\begin{array}{l}\text { Ik krijg voldoende } \\
\text { technische onders- } \\
\text { teuning om de <CoP } \\
\text { van de organisatie> } \\
\text { te kunnen gebruiken. } \\
\text { Bijv. door trainingen } \\
\text { en opleidingen. }\end{array}$ & $\begin{array}{l}\text { Facilitating } \\
\text { conditions }\end{array}$ & $\begin{array}{l}\text { Bhattacherjee, } \\
\text { (200 I);Venkatesh, } \\
\text { (2000) }\end{array}$ & Yes \\
\hline O7 & $\begin{array}{l}\text { I receive enough } \\
\text { support in the } \\
\text { way we can use } \\
\text { the digital tech- } \\
\text { nologies facili- } \\
\text { tating the <CoP } \\
\text { of the Organ- } \\
\text { isation>. E.g. } \\
\text { through training } \\
\text { and education. }\end{array}$ & $\begin{array}{l}\text { Ik krijg voldoende } \\
\text { ondersteuning in } \\
\text { de wijze waarop } \\
\text { we <CoP van de } \\
\text { organisatie> kun- } \\
\text { nen gebruiken. Bijv. } \\
\text { door trainingen en } \\
\text { opleidingen. }\end{array}$ & $\begin{array}{l}\text { Facilitating } \\
\text { conditions }\end{array}$ & $\begin{array}{l}\text { Bhattacherjee, } \\
\text { (200I);Venkatesh, } \\
\text { (2000) }\end{array}$ & Yes \\
\hline
\end{tabular}




\begin{tabular}{llll} 
\# $\begin{array}{l}\text { English trans- } \\
\text { lation of the } \\
\text { items: }\end{array}$ & $\begin{array}{l}\text { Original items } \\
\text { in Dutch: }\end{array}$ & $\begin{array}{l}\text { Concept: } \\
\text { itemted from or } \\
\text { based on scales or } \\
\text { findings from: }\end{array}$ & $\begin{array}{l}\text { Includ- } \\
\text { ed in } \\
\text { scale }\end{array}$ \\
\hline
\end{tabular}

$\begin{array}{llll}\text { Al I am able to share } & \mathrm{lk} \text { ben in staat } \quad \text { Ability } & \text { Siemsen et al (2008) } & \text { Yes } \\ \text { my knowledge, } & \text { om mijn kennis, } & & \\ \text { experiences and } & \text { ervaringen en in- } & & \\ \text { insights with } & \text { zichten te delen } & \\ \text { others } & \text { met anderen } & \end{array}$

A2 I am able to $\quad \mathrm{k}$ ben in staat Ability Turner \& Pennington Yes answer questions, om vragen te give advice or beantwoorden, provide examples advies te geven for questions or of voorbeelden requests from te verstrekken bij others vragen of verzoeken van anderen
A3 I am able to ex- $\quad \mathrm{k}$ ben in staat Ability Turner \& Pennington Yes press myself well om me goed in words uit te drukken in woord en geschrift

A4 I am able to
respond sensibly to messages from others

A5 I have no difficulty to contact colleagues who are known to me, directly via internet

A6 I have no difficulty to contact colleagues who are unknown to me, directly via internet $\mathrm{lk}$ ben in staat om inhoudelijk zinvol te reageren op berichten van anderen

Ik heb er geen moeite mee om online contact te leggen met voor mij bekende collega's

Ik heb er geen moeite mee om online contact te leggen met voor mij onbekende collega's
Ability Turner \& Pennington Yes (2015)

Ability Cadiz et al. (2009) Yes

Ability Cadiz et al. (2009) Yes 


\begin{tabular}{|c|c|c|c|c|c|}
\hline \# & $\begin{array}{l}\text { English trans- } \\
\text { lation of the } \\
\text { items: }\end{array}$ & $\begin{array}{l}\text { Original items } \\
\text { in Dutch: }\end{array}$ & Concept: & $\begin{array}{l}\text { Adapted from or } \\
\text { based on scales or } \\
\text { findings from: }\end{array}$ & $\begin{array}{l}\text { Includ- } \\
\text { ed in } \\
\text { scale }\end{array}$ \\
\hline A7 & $\begin{array}{l}\text { I have sufficient IT } \\
\text { skills to con- } \\
\text { nect online with } \\
\text { colleagues within } \\
\text { <CoP of the } \\
\text { organisation> }\end{array}$ & $\begin{array}{l}\text { Ik heb voldoende } \\
\text { ICT-vaardigheden } \\
\text { om online con- } \\
\text { tact te leggen } \\
\text { met collega's } \\
\text { binnen <CoP van } \\
\text { de organisatie> }\end{array}$ & Ability & Deursen et al., (20II) & Yes \\
\hline A8 & $\begin{array}{l}\text { I have sufficient } \\
\text { ICT skills to read } \\
\text { messages with- } \\
\text { in <CoP of the } \\
\text { organisation> }\end{array}$ & $\begin{array}{l}\text { Ik heb voldoende } \\
\text { ICT-vaardigheden } \\
\text { om berichten } \\
\text { te lezen binnen } \\
\text { <CoP van de } \\
\text { organisatie> }\end{array}$ & Ability & Deursen et al., (20II) & Yes \\
\hline A9 & $\begin{array}{l}\text { I have sufficient } \\
\text { ICT skills to share } \\
\text { messages with- } \\
\text { in <CoP of the } \\
\text { organisation> }\end{array}$ & $\begin{array}{l}\text { Ik heb voldoende } \\
\text { ICT-vaardigheden } \\
\text { om berichten } \\
\text { te delen binnen } \\
\text { <CoP van de } \\
\text { organisatie> }\end{array}$ & Ability & Deursen et al., (20II) & Yes \\
\hline
\end{tabular}

Items belonging to the communality

\begin{tabular}{llll} 
\# English & Original items in \\
translation of & Dutch: \\
the items: & & $\begin{array}{l}\text { Adapted from } \\
\text { or based on } \\
\text { scales or }\end{array}$ & $\begin{array}{l}\text { Includ- } \\
\text { scal in } \\
\text { findings from: }\end{array}$ \\
\hline
\end{tabular}

Cl In the group (s) in In de groep(en) Shared Chiu et al. (2006) Yes which I participate, waarin ik deelneem language we use common hanteren we jargon gemeenschappelijk jargon

C2 The members within the group

De leden - binnen

Shared Chiu et al. (2006) Yes (s) in which I de groep(en) language participate waarin ik deelneem discuss in an - discussiëren op understandable een begrijpelijke manier way 


\begin{tabular}{lllll}
\hline$\# \quad \begin{array}{l}\text { English } \\
\text { translation of } \\
\text { the items: }\end{array}$ & $\begin{array}{l}\text { Original items in Concept: } \\
\text { Dutch: }\end{array}$ & $\begin{array}{l}\text { Adapted from } \\
\text { or based on } \\
\text { scales or } \\
\text { findings from: }\end{array}$ & $\begin{array}{l}\text { Includ- } \\
\text { ed in } \\
\text { scale }\end{array}$ \\
\hline
\end{tabular}

C3 The members within the group

(s) in which I participate - use understandable language in their messages or articles

C4 There is no hierarchical ranking within the group (s) in which I participate

C5 There is equality within the group

(s) in which I participate, regardless of the 'titles' that others carry within the organisation

C6 There is not one person who makes all decisions within the group
(s) in which I participate

C7 There is not a feeling of "every man for himself" within the group (s) in which I participate

C8 In the group (s) in In de groep(en) which I participate, waarin ik we share common norms and values
De leden - binnen de groep(en) waarin ik deelneem - gebruiken begrijpelijke taal in hun berichten of artikelen

$\mathrm{Er}$ is geen hiërarchische rangorde binnen de groep(en) waarin ik deelneem

Er is gelijkheid binnen de groep(en) waarin ik deelneem, ongeacht de 'titels' die anderen binnen de organisatie dragen

$E r$ is niet één persoon die alle beslissingen neemt binnen de groep(en) waarin ik deelneem

Er heerst niet een gevoel van "ieder voor zich" binnen de groep(en) waarin ik deelneem deelneem delen we gezamenlijke normen en waarden
Shared language

Shared

Wood (2005) Yes leadership

Shared Wood (2005) Yes leadership

Shared

Wood (2005) No leadership

Shared Wood (2005) No leadership

Shared identity
Chiu et al. (2006) Yes 


\begin{tabular}{|c|c|c|c|c|c|}
\hline \# & $\begin{array}{l}\text { English } \\
\text { translation of } \\
\text { the items: }\end{array}$ & $\begin{array}{l}\text { Original items in } \\
\text { Dutch: }\end{array}$ & Concept: & $\begin{array}{l}\text { Adapted from } \\
\text { or based on } \\
\text { scales or } \\
\text { findings from: }\end{array}$ & $\begin{array}{l}\text { Includ } \\
\text { ed in } \\
\text { scale }\end{array}$ \\
\hline C9 & $\begin{array}{l}\text { In the group (s) in } \\
\text { which I participate } \\
\text { I feel a sense of } \\
\text { belonging }\end{array}$ & $\begin{array}{l}\text { In de groep(en) } \\
\text { waarin ik deelneem } \\
\text { heb ik het gevoel } \\
\text { dat ik erbij hoor }\end{array}$ & $\begin{array}{l}\text { Shared } \\
\text { identity }\end{array}$ & Chiu et al. (2006) & Yes \\
\hline
\end{tabular}


Appendix 10.2: Loadings and cross-loadings Model $\mathrm{I}^{6}$.

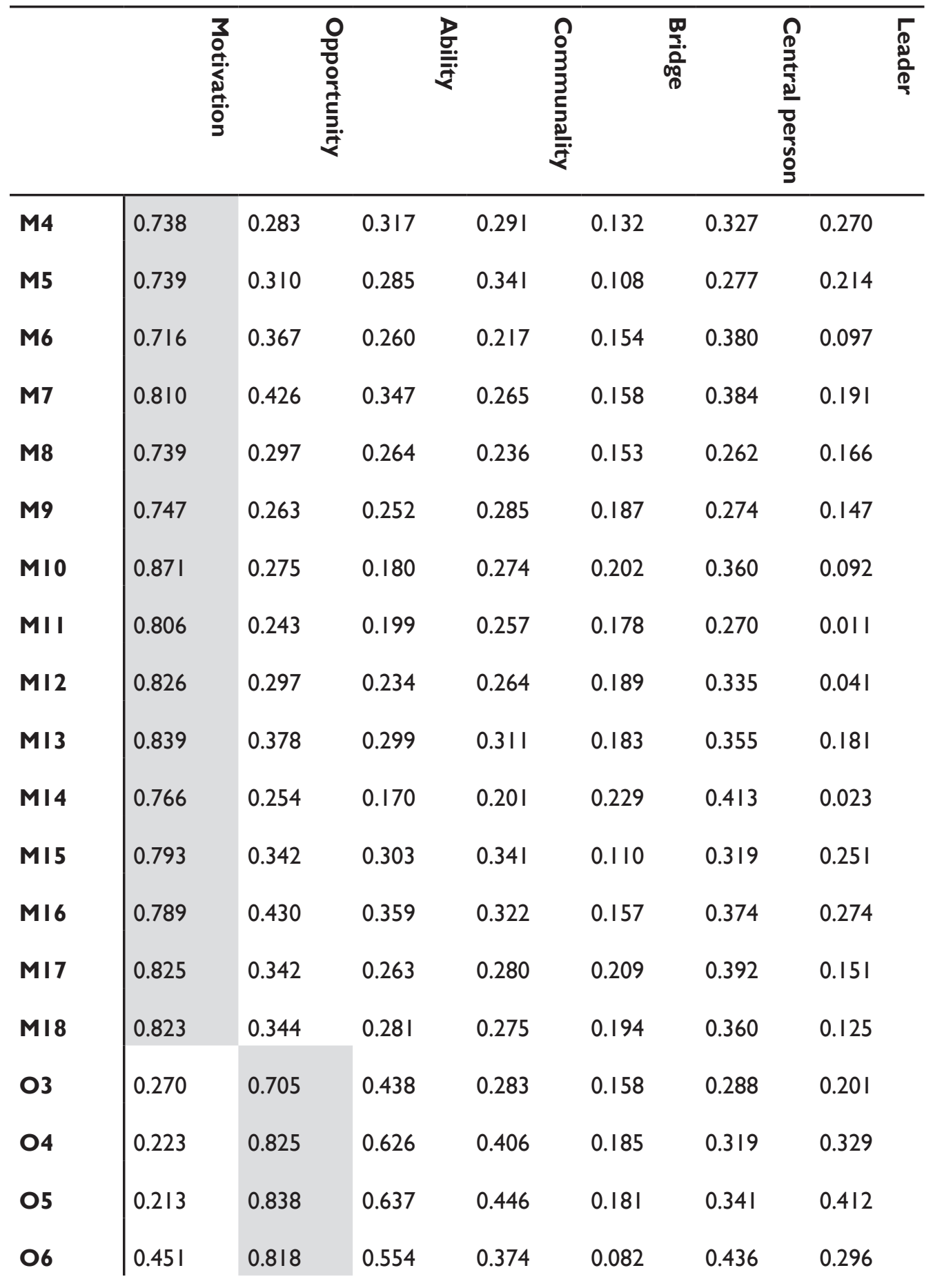

${ }^{6}$ Cross loadings have been measured for all constructs, but show comparable results of the acceptable 0.7 or higher (Kwong-Kay Wong, 2013) 


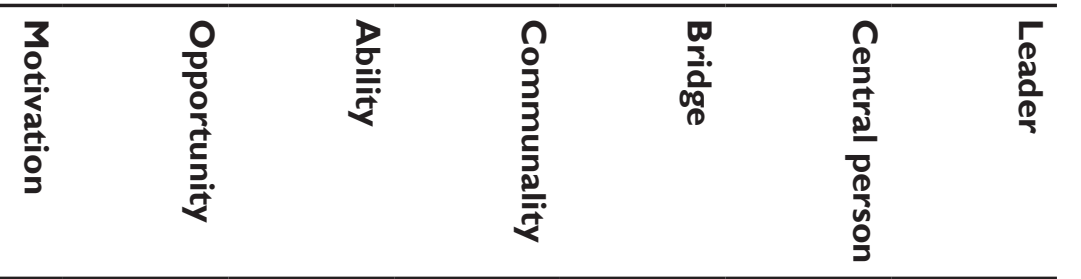

\begin{tabular}{|c|c|c|c|c|c|c|c|}
\hline 07 & 0.474 & 0.822 & 0.547 & 0.380 & 0.248 & 0.495 & 0.313 \\
\hline Al & 0.252 & 0.685 & 0.840 & 0.459 & 0.165 & 0.358 & 0.427 \\
\hline A2 & 0.294 & 0.658 & 0.837 & 0.387 & 0.176 & 0.371 & 0.321 \\
\hline A3 & 0.059 & 0.374 & 0.695 & 0.225 & 0.108 & 0.232 & 0.324 \\
\hline A4 & 0.168 & 0.443 & 0.739 & 0.315 & 0.130 & 0.266 & 0.359 \\
\hline A5 & 0.302 & 0.484 & 0.781 & 0.348 & 0.124 & 0.275 & 0.343 \\
\hline A6 & 0.391 & 0.499 & 0.730 & 0.381 & 0.160 & 0.336 & 0.262 \\
\hline A7 & 0.281 & 0.545 & 0.759 & 0.449 & 0.176 & 0.290 & 0.322 \\
\hline A8 & 0.335 & 0.604 & 0.833 & 0.328 & 0.186 & 0.326 & 0.259 \\
\hline A9 & 0.298 & 0.553 & 0.757 & 0.339 & 0.184 & 0.278 & 0.238 \\
\hline Cl & 0.274 & 0.400 & 0.374 & 0.808 & 0.123 & 0.254 & 0.271 \\
\hline $\mathrm{C} 2$ & 0.281 & 0.417 & 0.370 & 0.866 & 0.165 & 0.265 & 0.291 \\
\hline $\mathrm{C} 3$ & 0.265 & 0.433 & 0.440 & 0.870 & 0.146 & 0.243 & 0.345 \\
\hline C4 & 0.319 & 0.362 & 0.349 & 0.853 & 0.160 & 0.311 & 0.211 \\
\hline C5 & 0.288 & 0.378 & 0.388 & 0.876 & 0.162 & 0.292 & 0.308 \\
\hline C8 & 0.260 & 0.266 & 0.319 & 0.721 & 0.119 & 0.150 & 0.202 \\
\hline C9 & 0.346 & 0.458 & $0.45 I$ & 0.801 & 0.162 & 0.314 & 0.349 \\
\hline Bridge & 0.216 & 0.216 & 0.202 & 0.180 & 1.000 & 0.638 & 0.293 \\
\hline $\begin{array}{l}\text { Central } \\
\text { person }\end{array}$ & 0.436 & 0.477 & 0.395 & 0.322 & 0.638 & 1.000 & 0.416 \\
\hline Leader & 0.193 & 0.393 & 0.413 & 0.348 & 0.293 & 0.416 & 1.000 \\
\hline
\end{tabular}




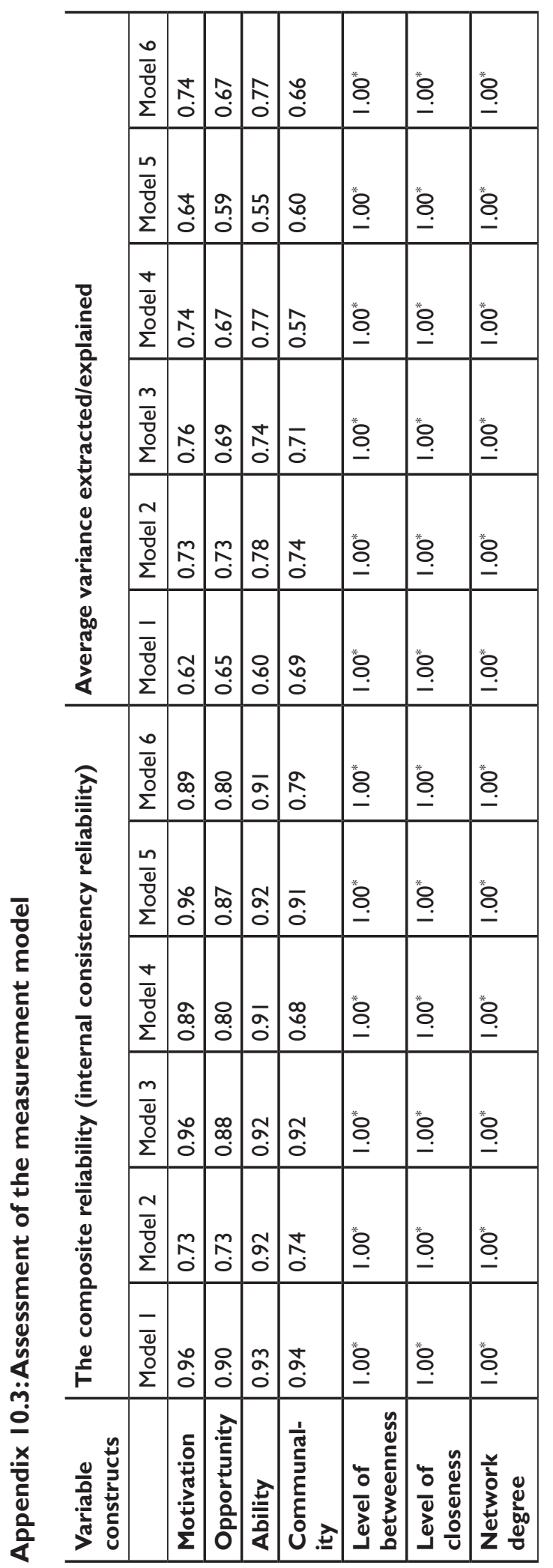





\section{Samenvatting (Dutch Summary)}

Om de ontwikkelingen in de kenniseconomie het hoofd te bieden zijn veel bedrijven en organisaties op zoek naar mogelijkheden om de kennisuitwisseling en samenwerking binnen en buiten de organisatie te optimaliseren. Eén van de methoden die veel wordt toegepast is het inrichten en onderhouden van een Community of Practice (CoP). Een CoP is een sociaal netwerk waar mensen in een organisatorische context samenkomen rond een gemeenschappelijk(e) onderwerp, passie of interesse en regelmatig on- en offline communiceren waarbij de focus ligt op kennisbeheer, innovatie, leren en sociaal netwerken.Veel academici hebben zich de afgelopen decennia al gebogen over deze CoPs, waarbij zij zich met name hebben gericht op de motivaties en barrières voor individuen om kennis daarbinnen te delen. In dit exploratieve onderzoek is een volgende stap gezet in het ontwikkelen van kennis en expertise rondom de ontwikkeling van deze CoPs vanuit verschillende perspectieven. Hierbij gaat het om het individueel sociaal gedrag en het groepsgedrag binnen deze CoPs. In aanvulling daarop is een monitoring en evaluatie methodologie ontwikkeld waarbij op een unieke manier verschillende databronnen gecombineerd en geïntegreerd zijn. Deze stap kenmerkt zich met name door de vraag hoe we met data gedreven technieken en daarop gebaseerde netwerkanalyses, ontwikkelingen in een CoP kunnen begrijpen en waar mogelijk sturen.

Hoofdstuk I schetst achtergronden die leiden tot de drie centrale onderzoeksvragen:

I. Welke individuele, organisatorische en community factoren beïnvloeden het sociale gedrag van community-leden in een community of practice?

2. Hoe ontwikkelt groepsgedrag zich in een community of practice?

3. Wat is een nuttige methodologie voor het monitoren en evalueren van sociaal gedrag in communities of practice?

De eerste vraag richt zich op individueel gedrag, de tweede op ontwikkelingen in het groepsgedrag en om deze gedragingen en ontwikkelingen inzichtelijk te maken is een samenhangende, geïntegreerde set van methoden en technieken gebruikt waarbij verschillende datasets zijn gekoppeld als antwoord op de derde onderzoeksvraag. Het doel hierbij is om een eerste stap te zetten naar een nuttige methodologie die voor zowel de academische wereld als de praktijk een nieuwe aanzet vormt voor het inzichtelijk maken van sociaal gedrag in CoPs.

Hoofdstuk 2 schetst het conceptueel kader waarbij de belangrijkste concepten in dit onderzoek in samenhang worden gedefinieerd. Het eerste centrale concept is continue professionalisering (continuing professional development) dat een steeds voornamer aandachtsgebied vormt voor organisaties. Hierbij wordt de continue ontwikkeling van de werknemer gezien als zeer belangrijk, het moet er namelijk voor zorgen dat individuen continu voorbereid zijn op nieuwe, markt beïnvloedende ontwikkelingen. Sociale interactie is een tweede concept dat een voorname rol speelt in dit onderzoek, met name doordat professionele ontwikkeling steeds vaker samengaat met sociaal leren (social learning), een concept dat gebaseerd is op het idee dat men van elkaar kan leren via allerlei vormen van sociale interacties. Om sociaal leren te faciliteren 
worden in toenemende mate communities of practice geïnitieerd, dit zijn sociale netwerken van gelijkgestemden die in een community infrastructuur (community infrastructure) samenkomen voor bijvoorbeeld kennisuitwisseling, samenwerking en sociaal netwerken. Een netwerk van factoren beïnvloeden het uiteindelijke gedrag van community leden binnen deze community infrastructuren, waardoor de community ontwikkeling niet eenvoudig inzichtelijk kan worden gemaakt. Om community ontwikkeling toch inzichtelijk te maken en tevens te verklaren is een community foundation framework ontwikkeld. Dit raamwerk bestaat uit de organisatiedoelen, community implementatie strategieën en de organisatiecultuur. Deze drie context-beschrijvende factoren dragen bij aan het inzichtelijk maken van de positie van de CoP binnen organisaties. Tot slot kunnen de community leden op basis van hun gedrag gekarakteriseerd worden in typen deelnemers gedifferentieerd in rollen en netwerkposities.

Hoofdstuk 3 bestaat uit een analyse van theoretische inzichten vanuit onder andere de onderzoeksgebieden communicatie, informatiemanagement en organisatiemanagement, waarbij de nadruk ligt op het gebruik van en deelname in sociale systemen zoals CoPs. De selectie van een passend model dat aansluit bij de doelen van dit onderzoek is uitgevoerd op basis van vier criteria: passend bij de context, toepasbaarheid in vergelijkbare situaties, nauwkeurig doch eenvoudig en toepasbaar op daadwerkelijk gedrag. Uit analyse van de literatuur is een hybride model voortgekomen dat gebaseerd is op het bestaande Motivation, Opportunity, Ability model (MOA), aangevuld met een vierde, contextuele factor: Communality (gemeenschappelijkheid). Aangezien het MOA-model een model is waarbij verschillende sociaalwetenschappelijke theorieën samenkomen, zijn verschillende modellen geëvalueerd om te bepalen in hoeverre bepaalde factoren alsnog opgenomen dienen te worden in het nieuwe MOAC-model. Het resultaat hiervan is een model waarbij de vier factoren uit het MOAC-model verder zijn uitgewerkt. Motivatie is bijvoorbeeld uitgewerkt in de motivatie om geïnformeerd te zijn, de motivatie om arbeidsprestaties te verbeteren, subjectieve normen en de motivatie om de sociale reputatie te verbeteren. Voor de opportunity geldt dat de geboden tijd door de organisatie, de mate van arbeidsautonomie en de faciliterende voorwaarden zijn gehanteerd als indicator voor de mate van opportunity. Het vermogen van een individu (ability) is initieel verder opgedeeld in de perceptie van het vermogen om jezelf uit te drukken, de perceptie van het vermogen om contact te leggen en de gepercipieerde digitale vaardigheden, ofwel selfefficacy. De communality is tot slot verder opgesplitst in de mate van een gedeelde identiteit, de mate van gedeeld leiderschap en de mate waarin een gedeelde taal wordt gesproken.

De kern van dit onderzoek draait om vier onderscheidende casussen die onderzocht zijn aan de hand van de volgende onderdelen en criteria: domein, omvang, organisatiedoelen, community implementatiestrategieën en organisatiecultuur. Deze vier casussen vormen de basis voor het onderzoek in de hoofdstukken 6, 7, 8, 10 en II. In hoofdstuk 9 worden deze vier casussen eenmalig aangevuld met drie extra casussen omdat hierin community ontwikkelingen vanuit een data-gedreven perspectief onderzocht worden waarbij grote(re) datasets gewenst zijn. Hoofdstuk 4 bestaat uit een analyse van deze casussen aan de hand van de eerdergenoemde criteria. De eerste casus richt zich met name op het aanbieden van financiële producten, deze internationale bank is internationaal georiënteerd maar heeft zich ook in Nederland gehuisvest. De deelnemers hebben dan ook allemaal hun standplaats in Nederland. De tweede casus vertegenwoordigt een groot Nederlands ziekenhuis, met een interdisciplinaire samenstelling van deelnemers. De derde casus is een gedecentraliseerde jeugdhulporganisatie waarbij de werknemers vooral op locatie werken en de vierde casus richt zich vooral op het ondersteunen van mensen met een beperking bij deelname in de samenleving. 
De derde onderzoeksvraag is gericht op het ontwikkelen van een community monitoring en evaluatie methodologie (CMEM). In Hoofdstuk 5 zijn de methoden en technieken, centraal in dit onderzoek, beschreven en in samenhang vormen ze het CMEM. Aan de hand van deze methode worden zowel onderzoekers als professionals in staat gesteld om op een zinvolle manier gebruik te maken van vernieuwende technieken om ontwikkelingen binnen CoPs te evalueren en monitoren. Om de bruikbaarheid van de methodiek te vergroten is ervoor gekozen om voor zover mogelijk gebruik te maken van web-based social interaction data. Deze data vormen een verzameling van de sociale interacties die individuen binnen CoPs kunnen aangaan, zoals het posten van een bericht of het becommentariëren van een bericht. Bij het verwerken van deze data wordt een typisch data gedreven proces gevolgd dat bestaat uit de stappen business understanding, data gathering, dataprocessing, data mining, pattern evaluation, knowledge presentation en strategic decision-making. Door deze stappen te volgen ontstaat een actueel en realistisch beeld van de ontwikkelingen binnen de CoPs.

Dit promotieonderzoek is gestart vanuit de wetenschappelijke en maatschappelijke behoefte om meer inzicht en expertise te genereren rondom de ontwikkeling van CoPs.Vanaf hoofdstuk 6 starten de studies die de basis vormen voor het begrijpen van deze ontwikkelingen in en van CoPs. Hoofdstuk 6 beschrijft een kwantitatieve studie naar de relatie tussen de individuele factoren motivatie (informing, work performance, social reputation en subjective norms) en individueel vermogen (ability: ability to express, ability to contact en digital skills) en het individueel sociaal gedrag. Individueel sociaal gedrag is hierbij opgesplitst in twee typen gedrag: kennisverwerving (knowledge acquisition behaviour) en sociale interactie (social interaction behaviour). Met behulp van een vragenljist is een onderzoek uitgevoerd onder community leden $(n=22 I)$ van vier verschillende CoPs in evenzoveel organisaties. Uit de factoranalyses in dit deel van het onderzoek is gebleken dat de initieel veronderstelde onderverdeling in het individueel vermogen niet geldt, waardoor het individueel vermogen als één variabele is onderzocht. De partial least squares structural equation modelling (PLS-SEM) methodologie is in deze studie en in die van hoofdstuk 7, 8 en 10 toegepast. Deze modelling techniek maakt het mogelijk om exploratieve modellen met een scheve verdeling te valideren. Het activiteitenniveau in de CoP is - zoals verwacht mag worden van CoPs - scheef verdeeld door de verschillende niveaus van sociale activiteit. De resultaten laten vervolgens zien dat er belangrijke relaties bestaan tussen de motivatie om geïnformeerd te blijven, de motivatie om arbeidsprestaties te verbeteren, de motivatie vanuit sociale druk (subjective norm) en het gerapporteerde individueel vermogen met daadwerkelijke kennisverwerving binnen de CoPs. Het aangaan van sociale interacties is met name gerelateerd aan de motivatie om werkprestaties te verbeteren, de motivatie om sociale reputatie te onderhouden en/of verbeteren en het gerapporteerde individueel vermogen tot zinvol gedrag.

Het perspectief waarbij specifiek organisatiefactoren worden getoetst staat centraal in hoofdstuk 7. Hierbij is de factor geboden mogelijkheden (opportunity) getoetst aan de hand van de drie onderliggende variabelen: geboden tijd (time), arbeidsautonomie (work autonomy) en de faciliterende voorwaarden (facilitating conditions) met kenniswerving en sociale interactie. In dit hoofdstuk is een deel uit de vragenlijst die beschreven is in hoofdstuk 6 geëvalueerd, hierbij gaat het als gezegd om de organisatiefactoren. Eén van de belangrijkste bevindingen is dat het individueel sociaal gedrag voornamelijk wordt beïnvloed door de mate waarin door de organisatie wordt voldaan aan de faciliterende voorwaarden. De mate waarin respondenten het gevoel hebben dat de organisatie hen faciliteert in deelname in de CoP, beïnvloedt de mate waarin zij daadwerkelijk in meer of mindere mate deel gaan nemen in de CoP. Ook is er een significante relatie gevonden tussen enerzijds de arbeidsautonomie en anderzijds 
het kennis verwervende gedrag. De mate waarin respondenten dus een verhoogde mate van arbeidsautonomie ervaren lijkt dus een indicatie te zijn voor de mate waarin ze kennis werven binnen de CoP.

In hoofdstuk 8 wordt het individueel gedrag getoetst aan de hand van specifieke communityfactoren. Hierbij ligt de focus op de ervaren mate van gemeenschappelijkheid (communality) dat onderdeel vormt van het MOAC model en in relatie is gebracht met de mate van kenniswerving en sociale interactie. Ook in dit hoofdstuk is gebruik gemaakt van een deel van de vragenlijst die in hoofdstuk 6 uitgebreid is beschreven. Onder de factor communality zijn verschillende variabelen opgenomen die primair gericht zijn op individuele percepties, hierbij gaat het om gedeelde identiteit (shared identity), gedeelde taal (shared language) en gedeeld leiderschap (shared leadership). Uit de resultaten blijkt dat de relatie tussen de mate van gedeelde identiteit een behoorlijk sterke relatie heeft met het individueel sociaal interactiegedrag. De relatie tussen de mate van gedeelde identiteit en het kennis verwervende gedrag is ook aanwezig, zij het in mindere mate. Dit impliceert dat community leden die een zekere mate van gedeelde identiteit ervaren, eerder geneigd zijn tot het aangaan van verschillende vormen van sociale interactie. De mate van gedeeld leiderschap is eveneens positief significant gerelateerd aan het sociale interactiegedrag.

In de voorgaande drie hoofdstukken is met name het perspectief gekozen van individueel sociaal gedrag.Vanaf hoofdstuk 9 ligt de nadruk op het groepsgedrag. In dit hoofdstuk is gekeken naar specifieke community ontwikkelingspatronen en de positie van de community rollen daarbinnen. De vier organisaties die de casuïstiek in de eerste studie hebben gevormd zijn ook in dit hoofdstuk onderzocht, aangevuld met drie extra casussen waarvan we de web-based social interaction data hebben geworven. In dit onderzoek zijn de web-based social interaction data gebruikt om de ontwikkeling van de CoPs in de eerste veertien maanden na introductie te evalueren. Web-based social interaction data representeren verzamelde en opgeslagen digitale sporen die community leden achterlaten binnen de CoPs, hierbij gaat het primair om events en acties zoals het creëren van een bericht, het becommentariëren van dit bericht tot het aangaan van één-op-één gesprekken Deze data kunnen op allerlei manieren gebruikt worden, maar in dit deel van het onderzoek ligt de focus op het ontsluiten van community ontwikkelingspatronen die leiden tot het identificeren en formaliseren van CoP-ontwikkelingen. Aan de hand van genormaliseerde scores zijn we in staat om een vergelijking te maken tussen de CoPs, maar ook om een algemene ontwikkeling inzichtelijk te maken van de opstartfase van de CoPs (eerste veertien maanden). De resultaten laten zien dat de gemiddelde CoP een ontwikkelingspatroon volgt dat begint met een rustige aanloop met lichte fluctuaties in de sociale activiteiten, waarna na een negental maanden een sterke stijging ontstaat in de acceptatie van de CoP.Vanaf dat moment lijken CoPs een nieuwe fase in te gaan die gekarakteriseerd kan worden als een volwassenheidsfase. De ontwikkelingen verschillen echter sterk per organisatie, twee organisaties laten een sterke community-gecentreerd ontwikkelingspatroon zien waarin veel verschillende vormen van sociale interactie worden aangegaan, maar de meerderheid (4/7) CoPs laten een sterke kennis gecentreerde ontwikkeling zien. Eén organisatie laat vooral een dialoog-gecentreerde ontwikkeling zien, waarbij individuen elkaar met name opzoeken. Aangaande de rollen die community deelnemers laten zien, zijn gewogen scores berekend voor de sociale interactietypen die zij aangaan. Hieruit zijn selecties voortgekomen van rollen onder de community deelnemers. Het resultaat is een onderverdeling van een selecte groep $(0,2 \%)$ community leaders (community manager), een aanzienlijke groep kennisdelers opgesplitst in posters $(5,0 \%)$ en commenters $(6,2 \%)$, een substantiële groep networkers $(5,0 \%)$, appreciators $(4,2 \%)$, chatters $(3,1 \%)$ en advisers $(0,5 \%)$, een grote meerderheid $(68,6 \%)$ uit information 
absorbers (kenniswervers) en tot slot is een kleine groep volledig inactief $(7,2 \%)$. In drie van de zeven gevallen nemen de community managers een prominente rol in tijdens de eerste maanden van de community-ontwikkeling. In de overige casussen zijn geen prominente rollen waarneembaar, maar nemen verschillende rollen een actievere positie in.

De sociale netwerkposities die individuen hebben binnen CoPs vormen het centrale thema in hoofdstuk 10. Hierbij is onderscheid gemaakt tussen drie dominante posities die volgens de literatuur met sociale netwerkanalyse technieken geïdentificeerd kunnen worden: de centraal gepositioneerde personen (degree centrality), de "brug" (betweenness centrality) en de leider (closeness centrality). Een aanzienlijk deel van de deelnemers $(n=144)$ aan de vragenlijst dat de basis vormt voor de hoofdstukken $6 \mathrm{t} / \mathrm{m} 8$ heeft aangegeven dat ze toestaan dat hun antwoorden gekoppeld mochten worden aan de web-based social interaction data. De netwerkposities van de individuele deelnemers zijn dus gebaseerd op de relatieve positie in de gehele CoP met alle sociale interacties. Het analyseren van de rol van de motivatie, opportunity, ability en communality op deze netwerkposities is gebaseerd op deze steekproef. In totaal zijn zes modellen ontwikkeld, (I) volledig model waarbij de focus ligt op de relatie tussen de MOACfactoren en de netwerkposities, (2) relatie tussen de MOAC-factoren en de netwerkposities in de eerste negen maanden (le fase) van de CoP, (3) relatie tussen de MOAC-factoren en de netwerkposities in de tweede negen maanden (2e fase) van de CoP, (4) volledig model waarbij de focus ligt op de relatie tussen de netwerkposities en de MOAC-factoren, (5) netwerkposities versus de MOAC-factoren in de eerste fase van de CoP en (6) netwerkposities versus de MOAC-factoren in de tweede fase van de CoP. Uit de resultaten van dit exploratieve onderzoek is gebleken dat in het eerste model een sterke relatie bestaat tussen de motivatie en geboden mogelijkheden en de centraal-gepositioneerde personen. Daarnaast is een significante relatie gevonden tussen de motivatie en leiders en tot slot laat het volledige model zien dat er een relatie is tussen het individueel vermogen en de mate van communality versus de personen met een brug slaande positie. Meest opvallende resultaat in dit onderzoek zijn die tussen de sociale netwerkposities en de kansen en mogelijkheden van het individu. Mensen die een meer centrale positie hebben binnen een CoP zijn positiever over de kansen die zij krijgen van bijvoorbeeld hun organisatie. Daarnaast hebben zij vaker het gevoel dat zij het individueel vermogen bezitten om een zinvolle bijdrage te leveren aan de CoP. Deze percepties van individuen over de factoren in het MOAC-model en hun feitelijke netwerkposities laten een sterk polariserende ontwikkeling zien, waarbij de afstand tussen de deelnemers alsmaar verder wordt vergroot. Een ontwikkeling die in toenemende mate kenmerkend is voor de onlinegemeenschappen. Een analyse van de twee fasen laat verder zien dat de motivatie, opportunity en communality steeds belangrijker worden voor de CoP-posities gedurende de ontwikkeling ervan. Vanuit het perspectief van de netwerkposities en de relaties ten opzichte van de MOAC-factoren kunnen we concluderen dat met name de centraal-gepositioneerde deelnemers en de leidende deelnemers positief significante relaties onderhouden. In dit geval gaat het voor de centraalgepositioneerde deelnemers om de factoren opportunity en communality, die in de eerste fase positief significante relaties vertonen. In de tweede fase zijn er positief significante relaties tussen de centraal-gepositioneerde deelnemers en alle vier factoren: motivation, opportunity, ability en communality. Voor de leidende deelnemers zijn met name ontwikkelingen te zien tussen de netwerkpositie en de individuele mogelijkheden en vermogens.

Door gedrag in een CoP te beschouwen vanuit een netwerkperspectief ontstaan nieuwe mogelijkheden om ernaar te kijken. In Hoofdstuk II is het gedrag gekwantificeerd aan de hand van tweeledige netwerken (bipartite of two-mode network graphs) waarbij community leden $(n=125)$ gekoppeld zijn aan verschillende typen sociale interacties kenmerkend voor een CoP, 
bijvoorbeeld het posten van een bericht of het becommentariëren van deze berichten. De daaruit voortvloeiende sociale interactiepatronen laten een negatieve spreiding van het type sociale activiteit (activity spread) zien, wat erop duidt dat community-leden veel verschillende typen sociale interacties aangaan. Deze bevinding laat zien dat veel deelnemers de technische mogelijkheden binnen de CoPs wel kennen, maar deze gezien de resultaten uit de eerdere hoofdstukken niet altijd evengoed benutten. Eén van de andere sociale interactiepatronen die naar voren komt in de resultaten is dat wanneer community-leden één type interactie aangaan het aannemelijk is dat zij ook andere typen sociale interacties aangaan (shared affiliation). Tot slot zijn de individuele motivatie, geboden mogelijkheden en het individueel vermogen positief gerelateerd aan de mate waarin verschillende typen sociale interactie worden aangegaan.

Op basis van de resultaten in dit proefschrift kunnen we enkele overkoepelende conclusies trekken. Hierbij nemen we de drie centrale onderzoeksvragen in dit onderzoek als basis. De eerste onderzoeksvraag luidt: Welke individuele, organisatorische en community factoren beïnvloeden het sociale gedrag van community-leden in een community of practice? De belangrijkste factoren die in dit onderzoek naar voren komen als beïnvloedende factoren voor het individueel sociaal gedrag zijn de individuele factoren: motivatie om arbeidsprestatie te willen verbeteren aan de hand van kennis en expertise die gedeeld wordt binnen de CoP. Verder is het individueel vermogen van het individu - geoperationaliseerd als self-efficacy een belangrijke factor die het individueel sociaal gedrag beïnvloedt. De organisatie-specifieke factoren zijn met name de door de organisatie geboden optimale faciliterende voorwaarden (technische en methodologische ondersteuning) die gerapporteerd worden als een belangrijke randvoorwaarde voor individueel sociaal gedrag. Tot slot vormt de specifieke community-factor gedeelde identiteit een belangrijke indicator voor de mate van individueel sociaal gedrag binnen de CoP.

In het tweede deel van het onderzoek ligt de focus op het groepsgedrag. Hierbij behorend is de volgende onderzoeksvraag geformuleerd: Hoe ontwikkelt groepsgedrag zich in een community of practice? Om deze vraag te beantwoorden is groepsgedrag vanuit drie perspectieven onderzocht: community ontwikkelingspatronen, community netwerkposities en sociale interactiepatronen. Deze drie perspectieven bieden alle drie een unieke kijk op groepsgedrag en ontwikkelingen binnen CoPs. Groepsgedrag beschouwend vanuit het perspectief van community ontwikkelingspatronen laten overwegend kennis gecentreerde patronen zien, waarbij de nadruk ligt op het transformeren van impliciete kennis naar expliciete kennis en het algemeen expliciteren van kennis. Binnen deze ontwikkelingen is met name een speciale rol weggelegd voor enkele centraal gepositioneerde personen, waardoor de door organisaties gewenste vermindering van de sociale afstanden binnen organisaties juist niet bereikt worden. De sociale afstand tussen de actieve deelnemers en de minder actieve deelnemers lijkt alleen maar groter te worden. Dit wordt ook weerspiegeld in de sociale interactiepatronen waarbij een shared affiliation pattern zichtbaar is. Dit patroon laat zien dat community leden die één gemeenschappelijke vorm van sociale interactie vertonen, vaak ook andere vergelijkbare vormen van sociale interactie aangaan. In aanvulling daarop zijn het met name de gemotiveerde personen, die voldoende door de organisatie geboden mogelijkheden ervaren en het gevoel hebben dat zij het individueel vermogen bezitten om zinvol sociaal gedrag te vertonen, die ook overeenkomstig sociaal gedrag laten zien.

Het laatste focusgebied in dit onderzoek richt zich op de ontwikkeling van een community monitoring en evaluatie methodologie (CMEM), hierbij staat de volgende onderzoeksvraag centraal: Wat is een nuttige methodologie voor het monitoren en evalueren van sociaal 
gedrag in communities of practice? Het CMEM makt gebruik van verschillende, onderling samenhangende en complementaire datasets om een analyse van individueel sociaal gedrag binnen CoPs en groepsgedrag vanuit verschillende perspectieven mogelijk te maken. In dit proefschrift is een vragenlijst ontwikkeld om inzicht te geven in het individueel sociaal gedrag, waarbij de vragenlijst - op geanonimiseerde basis - is gekoppeld aan de web-based social interaction data. Het resultaat is een eerste stap in de ontwikkeling van een methodologie waarbij op een vernieuwende manier, gebruikmakend van bestaande datasets die veelal worden gecreëerd binnen online community software, inzicht wordt geboden in de ontwikkelingen binnen CoPs. Door gebruik te maken van de vragenlijst waarin sociaalpsychologische vragen worden gesteld, ontstaan daarnaast unieke mogelijkheden om mogelijke verklaringen te bieden voor deze ontwikkelingen. Door op een meer gerichte manier gebruik te maken van de vaak al aanwezige bronnen en deze op een efficiënte manier te verrijken met nieuwe data (bijv. vragenlijsten), ontstaat daarmee een nuttige methodologie dat als eerste aanzet dient voor vervolgstappen.

Samengevat draagt dit onderzoek bij aan de expertise voor het ontwikkelen en onderhouden van CoPs en het begrijpen van het hoe en waarom van de ontwikkelingen erbinnen. Het CMEM draagt tot slot bij met een benadering voor overwegend data-gedreven monitoring en evaluatie van CoPs verrijkt met sociaalpsychologische verklarende variabelen. Op basis van deze aanpak gaan we voorbij de hype rondom CoPs in organisaties en zetten we een nieuwe stap richting het zo gericht mogelijk ontwikkelen van de CoPs. 


\section{Dankwoord (Acknowledgements)}

Een proefschrift is niet compleet zonder een woord van dank richting de personen die in meer of minder mate hebben bijgedragen aan de totstandkoming ervan. Het gehele proces kenmerkt zicht door verschillende stappen die worden doorlopen waarbij goede en minder goede momenten elkaar razendsnel opvolgen. Het was dan ook een intrigerend, interessant, lang en soms ook wel pittige route dat heeft geleid tot het document dat nu voor je ligt.

Allereerst wil ik mijn dank uitspreken richting mijn co-promotor en tevens dagelijks begeleider Sjoerd de Vries voor zijn onuitputtelijke bron van enthousiasme, creativiteit en visionaire blik op de kennismaatschappij waarin we leven, maar ook op de wetenschap zoals deze nu is en in de toekomst waarschijnlijk steeds meer zal zijn. Bovendien demonstreerde hij herhaaldelijk zijn ongekende oplossingsgerichte houding. Dit betreft niet alleen in inhoudelijke of methodologische oplossingen, maar ook zeker in het vinden van oplossingen bij de noodzakelijke contractverlengingen. Natuurlijk wil ik ook mijn promotoren bedanken. Jan van Dijk, bedankt voor het vertrouwen dat je in mij had, de verhelderende inzichten, de (positief) kritische feedback en de sterke onderbouwing vanuit eigen kennis en expertise. Wolfgang Ebbers, hoewel je pas in een later stadium bent aangesloten heb je met name vanuit theoretisch oogpunt waardevolle input kunnen leveren, bedankt daarvoor!

Uiteraard wil ik m'n dank zeker ook uitspreken richting 'Winkwaves' voor de geboden mogelijkheid om gebruik te maken van hun sociale technologie, maar ook voor het introduceren van de klanten. Allereerst Irene die een belangrijke rol heeft gespeeld in het eerste uur, later Mark voor zijn hulp en vernieuwende inzichten met betrekking tot digitale samenwerking en daarna Maaike voor haar ondersteuning en tussendoor René voor zijn inspirerende kijk op (online) samenwerking binnen organisaties. Daarnaast wil ik ook zeker mijn dankwoord richten aan Hans voor de technische ondersteuning bij de dataverzameling. Tot slot wil ik Marjolijn, Esther, Evelyn, Astrid en Rob bedanken voor hun ondersteuning vanuit de organisaties.

Daarnaast wil ik uiteraard al mijn (oud-)collega's bedanken. Zonder te veel op namen in te gaan wil ik in ieder geval mijn kamergenoten Rutger, Sikke en Susanne bedanken voor de mooie discussies, de flauwe grappen en de goede gesprekken. Robert en Joachim voor de prettige samenwerking in de verschillende projecten (EIT, gemeente, provincie, etc.). De overige collega's voor de goede samenwerkingen, lunchwandelingen, afdelingsuitjes en mooie gesprekken.

Verder wil ik de verschillende projectpartners in Veldwerk, ODS, Transit, EIT Digital, ICW2020, Saxion Hogeschool, Gemeente Enschede en de Provincie Overijssel bedanken voor de mooie samenwerkingen. Projecten die vaak in samenstelling, omvang en inhoud fundamenteel van elkaar verschilden, maar waarbij één gemenedeler fier overeind bleef staan: samenwerking! Professionals uit verschillende domeinen werk(t)en in deze projecten samen aan de ontwikkeling en/of verbetering van producten en diensten die de wereld vaak weer een stukje beter maken. Deze projecten droegen niet altijd evengoed bij aan de totstandkoming van mijn proefschrift, maar hebben er uiteindelijk ook altijd voor gezorgd dat ik de door mij gewenste binding met 
de praktijk niet zou verliezen. Op allerlei niveaus heb ik een heleboel bijgeleerd, het gaat echter te ver om iedereen hiervoor individueel te bedanken, maar vanuit mijn optiek was het in ieder geval bijzonder waardevol.

Als vanzelfsprekend wil ik ook m'n vrienden bedanken voor de welkome afleiding. Inspanning moet uiteraard afgewisseld worden met ontspanning, daarom zijn de nodige speciaalbieravonden georganiseerd, bezoekjes aan de kroeg gebracht en het jaarlijkse mannenweekend mag ook niet meer op de agenda ontbreken.Als je het mij vraagt is het wederzijdse respect voor hetgeen we allemaal doen, denken en zeggen een cruciale voorwaarde voor het feit dat deze vriendengroep al zo'n beetje 25 jaar bestaat. Luuk, Twan, Wouter, Karel en Jordy, bedankt!

Mijn schoonfamilie: Gerda, Gerard, ook jullie bedankt voor jullie steun. Met name de interesse in het onderzoek, de gezellige familiebezoeken en jullie verdere hulp in de afgelopen jaren zijn ongekend. Hetzelfde geldt voor Sharona en Gidon. Ook jullie bedankt voor jullie interesse, steun en gezellige momenten!

Uiteraard kan mijn eigen familie ook niet ontbreken in dit stuk. Pap, mam, bedankt voor jullie vertrouwen, interesse en ondersteuning. Jullie hebben mij geleerd dat het met name uit jezelf moet komen als je iets wil bereiken, het gaat daarbij om doorzettingsvermogen, een nuchtere kijk op het leven, zelfstandigheid en vertrouwen. Eenzelfde vorm van dank ben ik ook verschuldigd aan 'mien breurs' René en Erik en zussen Chantal en Ellen. Ook zwagers - Merijn en Arjan - en schoonzussen - Ellen en Anneke - mogen hierbij niet ontbreken. De jaarlijkse ski-weekenden (zonder ski's), bbq's, bezoekjes aan voetbalwedstrijden en gezellige avonden zijn absoluut onmisbaar. Anneke, ik wil jou nog specifiek bedanken voor de ondersteuning bij de opmaak van het binnenwerk van dit proefschrift.

Tot slot, Maris! Zonder jouw onvoorwaardelijke steun, je niet aflatende enthousiasme, je flexibiliteit, je vertrouwen, liefde en je capaciteit om mij te motiveren wanneer dat nodig was. Je wist (en weet) altijd de juiste snaar te raken en cijfert jezelf daarbij meermaals weg, je bent geweldig! 


\section{About the Author}

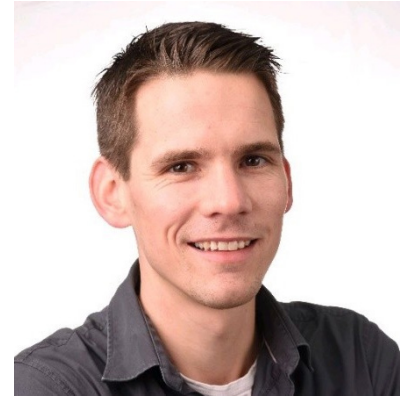
on social network analysis, data-driven research methods, user-centered design, and digital marketing.

In 20I4, he officially started his PhD research, guided by prof. dr. Jan van Dijk, prof. dr.Wolfgang Ebbers, and dr. Sjoerd de Vries. The PhD-project has been carried out in close cooperation with European, national, regional, and local projects.

Currently, Wouter works as a researcher at the research group Communication Science at the University of Twente. His research interests are in the field of data-driven research, smart monitoring and evaluation methodologies, and communal and networked environments.

Wouter Vollenbroek (1988) received his Masters degree in Communication Studies at the University of Twente in 2012 and then worked at the same university on various national and international research and consultancy projects. The emphasis in these projects was on the development, implementation and optimization of learning and working in networked environments (e.g. Communities of Practice, Communities of Learning, learning networks, and collaboration networks). In addition to this, Wouter was involved as a teacher and tutor in organization communication and business administration courses, especially those focused 


\section{Propositions}

\section{COMMUNITIES OF PRACTICE: BEYOND THE HYPE?}

\section{Wouter Vollenbroek}

I. A thriving community of practice highly depends on a small group of very active participants (this thesis)

2. The focus in a community of practice is primarily on knowledge management instead of on collaboration (this thesis)

3. To increase impact of communities of practice, community managers have to focus on continuous improving quality instead of social activity (this thesis)

4. Searching information is the foundation of participation in communities of practice (this thesis)

5. Impact of a community of practice will profit from increased clarity in the community of practice goals

6. Despite all the scientific and practical attention in the last two decades, the development of Communities of Practice in organisations do not exceed the experimentation stage

7. The enormous growth of the availability of real-time data improves efficient theory testing

8. Adequate insight into innovative knowledge management systems such as communities of practice requires a holistic approach in which complementary research methods are combined

9. The essence of communication research is interdisciplinarity with other research areas

10. The level of society is lost in a desire to develop 'communities' at the meso-level 


\section{UNIVERSITY OF TWENTE.}

Rapid technological advances, ever-changing markets in which increasingly high-quality standards are set, globalization and a growing need for individual personal development are only four developments with high impact on the development of organisations. The result is an organisation that continuously adapts to changing circumstances at the individual, organisational and group levels, with a contnuous impact on strategic and social movements in the organisation. These developments are visible for a couple of years now and have resulted in different organisational responses.

One main response is a transition towards a knowledge-driven business strategy which focuses on collaboration, knowledge exchange and social networking in communities of practice. A community of practice is a social network where people in an organisational context come together around a common topic, passion or interest and regularly interact on- and offline with a focus on knowledge management, innovation, learning and social networking.

The predominantly exploratory studies presented in this dissertation focus on questions of how and why knowledge management and strategies for the continuing professional development of individuals and groups in CoPs develop, in the face of technological advances and societal challenges. 